\title{
SOBRE A FORMULAÇÃO DE UM MODELO DE DANO PARA O CONCRETO
}

JOSÉ JULIO DE CERQUEIRA PITUBA

Tese apresentada à Escola de Engenharia de São Carlos da Universidade de São Paulo, como parte dos requisitos para a obtenção do título de Doutor em Engenharia de Estruturas.

ORIENTADOR: PROF. ASSOC. SERGIO PERSIVAL BARONCINI PROENÇA

São Carlos

2003 
À minha esposa, Gabriela e filha, Júlia Maria, com amor. 


\section{AGRADECIMENTOS}

Ao Prof. Dr. Sergio Persival Baroncini Proença, pela orientação, paciência e apoio demonstrados durante a realização deste trabalho.

Aos amigos do departamento de estruturas pela boa convivência durante todos esses anos, em especial aos amigos Romel Dias e Alexandre Marques pela ajuda e amizade nas horas alegres e difíceis.

Aos funcionários do Departamento de Estruturas pela pronta colaboração quando solicitada.

À Ana Rita Cordeiro da Silva pela grande ajuda durante a realização de ensaios no laboratório do Departamento de Estruturas.

Ao LMT (Laboratoire de Mécanique et Technologie - Université Paris VI) pela utilização do programa EFICoS.

À CAPES, pelo apoio financeiro que possibilitou a realização deste trabalho.

Agradeço aos meus pais e às minhas irmãs pelo amor, carinho e força.

Agradeço, finalmente, à Gabriela, pela paciência, amor, compreensão e ajuda nos momentos difíceis durante o desenvolvimento e conclusão deste trabalho.

A DEUS, por tudo. 
LISTA DE FIGURAS

LISTA DE TABELAS vi

LISTA DE SÍMBOLOS vii

RESUMO xiv

ABSTRACT Xv

1 INTRODUÇÃO 1

1.1 CONSIDERAÇÕES PRELIMINARES E SÍNTESE DO CONTEÚDO 1

1.2 COMENTÁRIOS SOBRE A MECÂNICA DO DANO NO CONTÍNUO 3

1.2.1 Introdução 3

1.2.2 Tensor Geral de Dano e as Equivalências de Deformação, Tensão e 4

Energia

1.3 COMPORTAMENTO MECÂNICO DO CONCRETO 14

$\begin{array}{ll}\text { 1.3.1 Introdução } & 14\end{array}$

1.3.2 Mecanismos de Danificação do Concreto 14

1.3.3 Comportamento Macroscópico do Concreto em Compressão Uniaxial 17

1.3.4 Comportamento Macroscópico do Concreto em Tração Uniaxial 21

1.3.5 Comportamento Macroscópico do Concreto em Solicitações Multiaxiais 24

1.3.5.1 Modos de danificação com vistas às solicitações multiaxiais 24

1.3.5.2 Solicitações - Tipo A 25

1.3.5.3 Solicitações - Tipo B 26

1.3.5.4 Solicitação - Tipo C 29

1.3.5.5 Modos de Ruptura 32

1.3.5.6 Mudanças de Volume 33

1.3.6 Influência das Condições de Ensaio 34

1.3.7 Influência da Trajetória de Carregamento 35

1.3.8 Comportamento do Concreto Fissurado em Relação à Resistência ao 37

Cisalhamento 
2 MEIOS ELÁSTICOS DANFICADOS ANISÓTROPOS COM RESPOSTA BIMODULAR

2.1 INTRODUÇÃO 39

2.2 ELASTICIDADE LINEAR DE MEIOS COM ANISOTROPIA E 40 RESPOSTA BIMODULAR

2.3 PROPOSTA DE FORMULAÇÃO PARA MEIOS ELÁSTICOS

ANISÓTROPOS COM DANIFICAÇÃO E RESPOSTA BIMODULAR

2.3.1 Caso Particular de Isotropia Transversal Induzida pela Danificação

2.4 MODELOS DE DANO PARA O CONCRETO

2.4.1 Introdução

2.4.2 Modelos Isótropos

2.4.3 Modelos com Anisotropia Induzida pelo Dano

3 PROPOSTA DE MODELO PARA O CONCRETO

3.1 INTRODUÇÃO

3.2 TENSOR CONSTITUTIVO CONSISTENTE COM O PRINCÍPIO DE EQUIVALÊNCIA DE ENERGIA

3.3 FORMA GERAL DO MODELO

3.4 CRITÉRIO PARA A DIVISÃO DO ESPAÇO DAS DEFORMAÇÕES 69

3.5 CRITÉRIOS DE DANIFICAÇÃO

3.5.1 Critério para Início de Dano

3.5.2 Critério de Carregamento-Descarregamento do Meio Danificado

3.6 LEIS DE EVOLUÇÃO 79

3.7 CRITÉRIO PARA A DEFINIÇÃO DO PLANO LOCAL DE ISOTROPIA TRANSVERSAL DO MATERIAL

3.8 PROPOSTA DE MODELO DE DANO COM DEFORMAÇÕES ANELÁSTICAS - VERSÃO UNIAXIAL

4 IDENTIFICAÇÃO PARAMÉTRICA DO MODELO PROPOSTO

4.1 INTRODUÇÃO 
4.3 ANÁLISE DA INFLUÊNCIA PARAMÉTRICA DO MODELO NA

RESPOSTA TENSÃO-DEFORMAÇÃO

4.4 RESULTDOS NUMÉRICOS DA CALIBRAÇÂO

101

4.4.1 Modelo de Dano Proposto (sem deformações permanentes) 101

4.4.2 Emprego da Versão Uniaxial do Modelo de Dano Proposto 107

Considerando-se Deformações Anelásticas

5 EXEMPLOS DE APLICAÇÃO DO MODELO PROPOSTO

5.1 INTRODUÇÃO

5.2 ANÁLISES UNIDIMENSIONAIS

5.2.1 Vigas em Concreto Armado [ÁLVARES (1993)]

5.2.2 Pórtico em Concreto Armado [VECCHIO \& EMARA (1992)]

5.3 ANÁLISES BIDIMENSIONAIS

5.3.1 Viga em Concreto Armado [PEREGO (1989)]

5.3.2 Vigas em Concreto Armado [ÁLVARES (1993)]

6 CONSIDERAÇÕES FINAIS, CONCLUSÕES E PERSPECTIVAS

APÊNDICE - Relações Matemáticas de Interesse

2 TENSORES ISÓTROPOS DE QUARTA ORDEM

3 PRODUTOS TENSORIAIS ENVOLVENDO O TENSOR A A-4 


\section{LISTA DE FIGURAS}

1.1 a) Representação simbólica de esforço e deformação nominais e efetivas no caso uniaxial

b) Elemento de volume representativo do corpo sob tração uniaxial

1.2 Hipótese de deformação equivalente

1.3 Hipótese de tensão equivalente

1.4 Defeitos sob partículas de agregados [VAN MIER (1984)]

1.5 Danificação em tração com início na zona de interface [MAZARS

1.6 Modos de abertura

1.7 Comportamento de isotropia transversal em tração uniaxial no concreto

1.8 Danificação em compressão com início na zona de interface

[BUYUKOZTURK, NILSON \& SLATE (1971)]

1.9 Diagrama tensão-deformação em compressão [PROENÇA (1988)]

1.10 Comportamento de isotropia transversal em compressão uniaxial no concreto

1.11 Deformação volumétrica e área específica de superfície de fissura versus tensão axial aplicada [STROEVEN (1979)]

1.12 Variação do coeficiente de Poisson [PROENÇA (1988)]

1.13 Diagrama tensão-deformação em tração uniaxial [TERRIEN (1980)]

1.14 Análise do comportamento em tração segundo HILLERBORG \& PETERSSON (1981)

1.15 Influência de uma tensão de tração $\left(\sigma_{3}\right)$ sobre o comportamento de um cubo comprimido nas duas direções $\left(\sigma_{1}=\sigma_{2}\right)$ [MAZARS (1984)]

1.16 Influência da tensão de confinamento em ensaios triaxiais [PROENÇA (1988)] 
1.17 Curvas tensão-deformação para testes de deformação plana com aumento de confinamento $\sigma_{3}=\beta \sigma_{1}(\beta=0 ; 0,05 ; 0,10)$ [VAN MIER (1984)]

1.18 Curvas tensão-deformação para testes com diferentes níveis de tensão 28 intermediária. Testes com $\sigma_{3}=0$ e $\sigma_{3}=0,05 \sigma_{1}$ com carregamento paralelo à direção de moldagem [VAN MIER (1984)]

1.19 Influência da anisotropia inicial no comportamento tensão-deformação triaxial [VAN MER (1984)]

1.20 Evolução da variação volumétrica sob carregamento hidrostático segundo BALMER (1949)

1.21 Modos de ruptura de espécimes de concreto sujeitos a estados biaxiais de tensão [KUPFER, HILSDORF \& RUSCH (1969)]

1.22 Modelos de variação volumétrica para materiais granulares

1.23 Dois diagramas ilustrando a influência da trajetória do carregamento

1.24 Comportamento sob carregamento de compressão com restrição lateral $\left(\sigma_{2}=\sigma_{3}=29,30 \mathrm{MPa}\right)$ [BERTACCHI \& BELOTTI (1972)]

1.25 Mecanismo de engrenamento dos agregados para transferência de tensões de cisalhamento através da fissura [FIGUEIRAS (1983)] 1.26 Comparação de vários diagramas propostos para o módulo de elasticidade transversal do concreto fissurado [ASCE (1981) apud FIGUEIRAS (1983)]

2.1 Representação esquemática da subdivisão do espaço de deformações em subdomínios de compressão e de tração

2.2 Modelo de dano escalar: módulo de Young em função variável de dano $\mathrm{D}$

2.3 Modelo de dano escalar: coeficiente de Poisson em função variável de dano $\mathrm{D}\left(\mathrm{v}_{0}=0,2\right)$ 
2.4 Planos de isotropia transversal no caso de tração uniaxial no concreto

2.5 Planos de isotropia transversal no caso de compressão uniaxial no concreto

3.1 Hiperplano $g(\varepsilon)$ para o caso de material totalmente danificado

3.2 Diagrama tensão-deformação em compressão e tração uniaxiais

[MAZARS (1984)]

3.3 Diagrama tensão-deformação em compressão uniaxial com carga e descarga

3.4 Taxa de deformação plástica $\left(1^{\mathrm{a}}\right.$ Hipótese $)$

4.1 a) Influência de $\mathrm{Y}_{0 \mathrm{~T}}$ sobre o comportamento em tração

b) Influência de $A_{1}$ sobre o comportamento em tração

c) Influência de $B_{1}$ sobre o comportamento em tração

4.2 a) Influência de $Y_{0 C}$ sobre o comportamento em compressão

b) Influência de $A_{2}$ sobre o comportamento em compressão

c) Influência de $\mathrm{B}_{2}$ sobre o comportamento em compressão

4.3 a) Influência de $Y_{0 C}$ sobre o comportamento em compressão biaxial

b) Influência de $A_{3}$ sobre o comportamento em compressão biaxial

c) Influência de $\mathrm{B}_{3}$ sobre o comportamento em compressão biaxial

4.4 a) Influência de $\beta_{\mathrm{T}}$ sobre o comportamento em tração

b) Influência de $\beta_{\mathrm{C}}$ sobre o comportamento em compressão 
4.6 Simulação de testes uniaxiais e biaxiais [KUPFER, HILSDORF \&

RUSCH (1969)]. Resultados experimentais e numéricos

4.7 Curvas de tensão x deformação volumétrica em compressão uniaxial e biaxial [KUPFER, HILSDORF \& RUSCH (1969)]: Resultados experimentais e numéricos.

4.8 Simulação de testes uniaxiais e biaxiais [KUPFER, HILSDORF \& RUSCH (1969)]: Resultados experimentais e numéricos

4.9 Simulação de teste de compressão triaxial [VAN MIER (1984)]:

Resultados experimentais e numéricos

4.10 Simulação do teste de compressão uniaxial [KUPFER, HILSDORF \& RUSCH (1969)]. Resultados experimentais e numéricos

4.11 Curvas tensão x deformação volumétrica em compressão uniaxial 108 [KUPFER, HILSDORF \& RUSCH (1969)]: Resultados experimentais e numéricos

5.1 Elemento finito empregado

5.2 Geometria e armação das vigas

5.3 Identificação paramétrica em compressão uniaxial: vigas em concreto armado

5.4 Identificação paramétrica em tração uniaxial: vigas em concreto armado

5.5 Resultados numéricos da análise unidimensional - vigas $3 \phi 10.0 \mathrm{~mm}$

5.6 Resultados numéricos da análise unidimensional - vigas $5 \phi 10.0 \mathrm{~mm}$

5.7 Resultados numéricos da análise unidimensional - vigas $7 \phi 10.0 \mathrm{~mm}$

5.8 Distribuição da danificação ao longo da seção transversal localizada no meio do vão (viga $3 \phi 10.0 \mathrm{~mm}-\mathrm{P}=30 \mathrm{kN}$ ) 
5.10 Identificação paramétrica em compressão uniaxial: pórtico em concreto armado

5.11 Identificação paramétrica em tração uniaxial: pórtico em concreto armado

5.12 Resultados numéricos do pórtico em concreto armado

5.13 Discretização em elementos finitos bidimensionais

5.14 Viga em concreto armado: geometria, simetria geométrica e de carregamento e disposição da armadura

5.15 Resultados numéricos - viga $6 \phi 32.0 \mathrm{~mm}$ : curva carga-deslocamento no meio do vão

5.16 Viga em concreto armado - viga $6 \$ 32.0 \mathrm{~mm}$ : distribuição de dano $\mathrm{D}_{1}$

5.17 Viga em concreto armado - viga $6 \$ 32.0 \mathrm{~mm}$ : distribuição de dano $\mathrm{D}_{2}$

5.18 Viga em concreto armado - viga $6 \phi 32.0 \mathrm{~mm}$ : distribuição de dano $\mathrm{D}_{3}$

5.19 Configurações das malhas com 342 e 7200 elementos finitos

5.20 Resultados numéricos (7200elementos) - viga $3 \phi 10 \mathrm{~mm}$

5.21 Resultados numéricos (7200elementos) - viga 5\$10 mm

5.22 Resultados numéricos (7200elementos) - viga 7\$10 mm

5.23 Viga em concreto armado - viga $7 \phi 10 \mathrm{~mm}$ : distribuição de dano $\mathrm{D}_{1}$

5.24 Viga em concreto armado - viga $7 \phi 10 \mathrm{~mm}$ : distribuição de dano $\mathrm{D}_{2}$

5.25 Viga em concreto armado - viga $7 \phi 10 \mathrm{~mm}$ : distribuição de dano $\mathrm{D}_{3}$

Figura 1 - Sistema local de referência do material. O plano YZ coincide com o plano local de isotropia transversal.

Figura 2 - Sistema local de referência do material. O plano local de isotropia transversal rotaciona em torno do eixo $\mathrm{Z}$. 
1.1 Classificação dos comportamentos relacionando o modo de danificação e o 31 tipo de solicitação [MAZARS (1984)]

5.1 Parâmetros dos modelos de Mazars, de La Borderie e do proposto.

5.2 Parâmetros dos modelos de Mazars, de La Borderie e do proposto.

5.3 Parâmetros dos modelos de Mazars [PEREGO (1989)] e proposto 124

5.4 Parâmetros do modelo proposto 


\section{LISTA DE SÍMBOLOS}

\section{GREGOS}

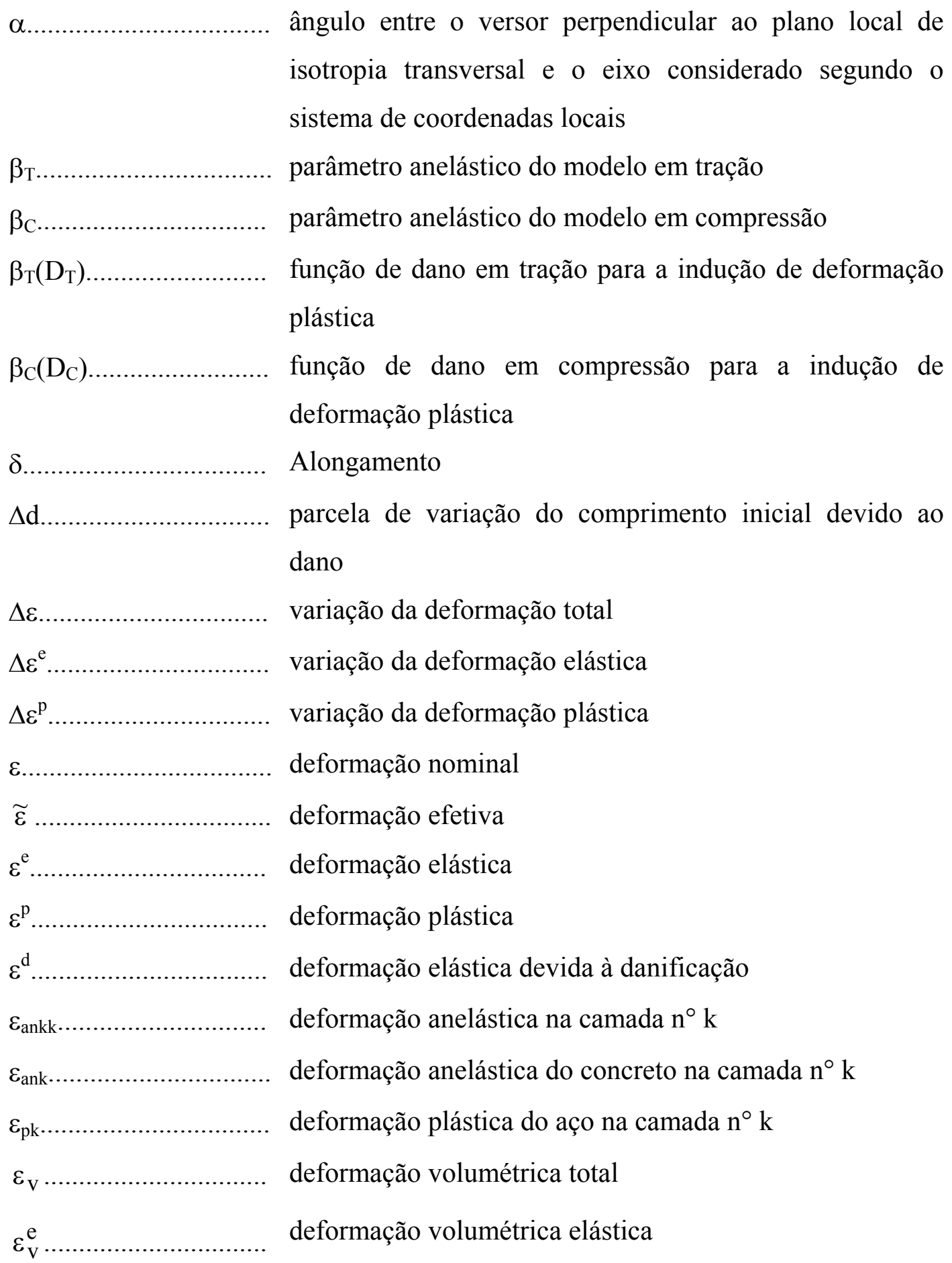




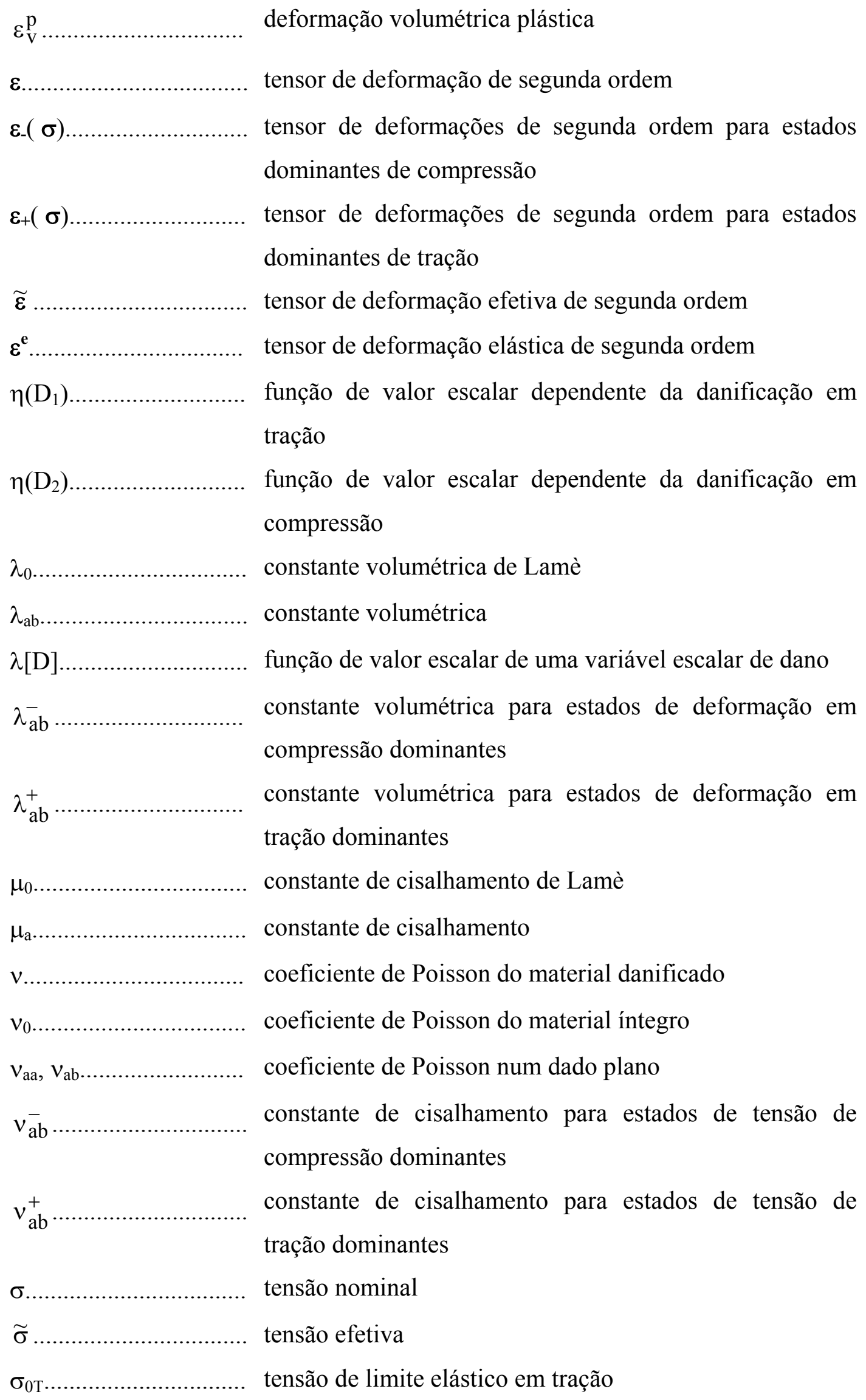




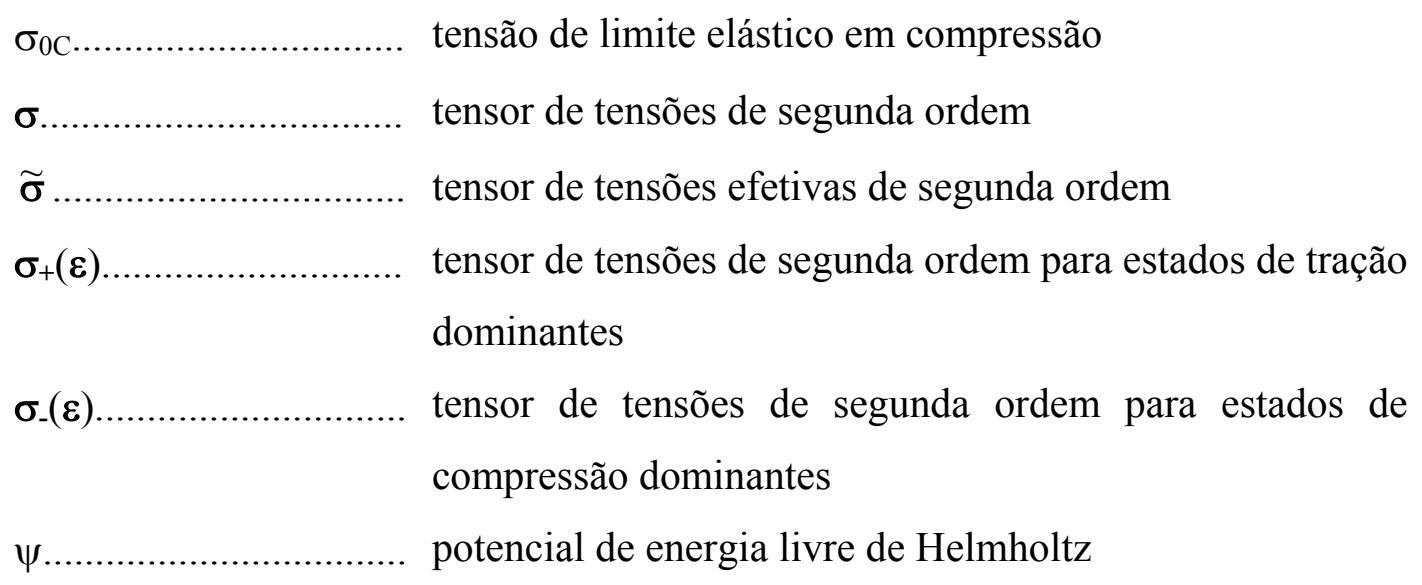

\section{ROMANOS}

a.

$\mathbf{a}^{\mathbf{T}}$

$\mathrm{A}_{1}, \mathrm{~B}_{1}, \mathrm{Y}_{01}$

$\mathrm{A}_{2}, \mathrm{~B}_{2}, \mathrm{Y}_{02}$

A.

B.

C.

$\mathrm{C}_{0}$

$\mathbf{C}_{\mathbf{T}}$

$\mathbf{C}_{\mathbf{C}}$

$\mathbf{C}_{0}-(\sigma)$ versor perpendicular ao plano de isotropia transversal vetor dos parâmetros a serem identificados parâmetros do modelo relativos à tração parâmetros do modelo relativos à compressão tensor de segunda ordem formado pelo produto tensorial do versor perpendicular ao plano local de isotropia transversal por ele mesmo tensor de segunda ordem associado a uma direção para o caso de ortotropia

tensor constitutivo de flexibilidade elástica de quarta ordem do material danificado tensor constitutivo de flexibilidade elástica isótropo de quarta ordem do material íntegro tensor constitutivo de flexibilidade elástica de quarta ordem do material danificado em regimes de tração tensor constitutivo de flexibilidade elástica de quarta ordem do material danificado em regimes de compressão tensor constitutivo de flexibilidade elástica isótropo de quarta ordem para estados dominantes de compressão 


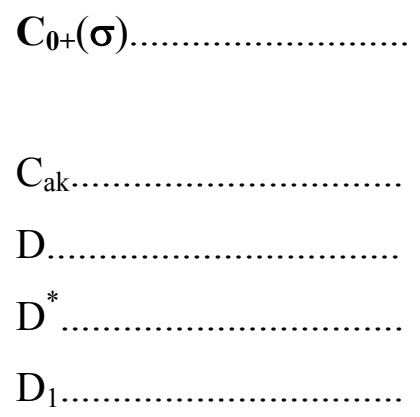

$\mathrm{D}_{2}$

$\mathrm{D}_{3}$

$\mathrm{D}_{4}$

$\mathrm{D}_{5}$

D

$\overline{\mathbf{D}}$

$\mathbf{D}_{\mathrm{T}}$

$\mathbf{D}_{\mathbf{C}}$

$\mathrm{e}(\sigma)$

E

$\mathrm{E}_{0}$

E.

$\mathbf{E}_{0}$

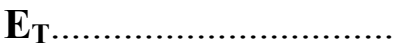

tensor constitutivo de flexibilidade elástica isótropo de quarta ordem para estados dominantes de tração concentração volumétrica de aço na camada $\mathrm{n}^{\circ} \mathrm{k}$ variável escalar de dano variável escalar alternativa de dano variável escalar representativa da danificação na direção perpendicular ao plano local de isotropia transversal em regimes de tração predominantes variável escalar representativa da danificação na direção perpendicular ao plano local de isotropia transversal em regimes de compressão predominantes variável escalar representativa da danificação no plano local de isotropia transversal em regimes de compressão predominantes

variável escalar representativa da danificação gerada pelo cisalhamento em regimes de tração predominantes variável escalar representativa da danificação gerada pelo cisalhamento em regimes de compressão predominantes tensor de dano de quarta ordem tensor de dano de oitava ordem tensor de dano de quarta ordem em tração tensor de dano de quarta ordem em compressão valor do salto do tensor constitutivo de flexibilidade elástica de quarta ordem módulo de elasticidade do material danificado módulo de elasticidade do material íntegro tensor constitutivo de rigidez elástica isótropo de quarta ordem do material danificado tensor constitutivo de rigidez elástica isótropo de quarta ordem do material íntegro tensor constitutivo de rigidez elástica de quarta ordem do material danificado em regime de tração 
$\mathbf{E}_{\mathbf{C}}$

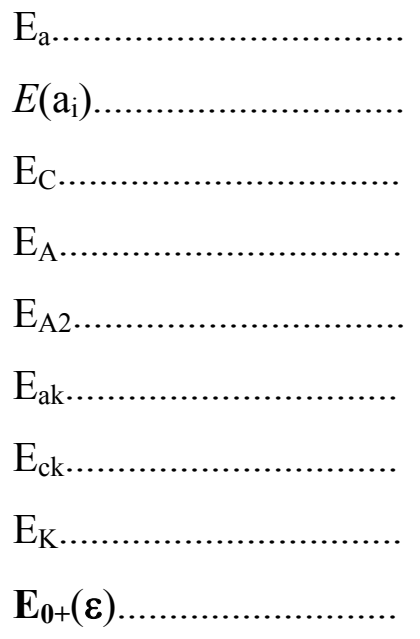

$\mathbf{E}_{0}-(\varepsilon)$

F

$\mathrm{f}_{\mathrm{T}}$

$\mathrm{f}_{\mathrm{C}}$.

$\mathrm{f}_{\mathrm{T}}(\sigma)$

$f_{C}(\sigma)$

G.

$\mathrm{G}_{0}$

$\mathrm{g}_{\mathrm{T}}$.

$\mathrm{g}_{\mathrm{C}}$

$\mathrm{g}(\varepsilon)$

$h(\sigma)$

I.

$\mathrm{I}_{1}$

II. tensor constitutivo de rigidez elástica de quarta ordem do material danificado em regime de compressão módulo de elasticidade numa dada direção função erro da formulação de mínimos quadrados módulo de elasticidade do concreto módulo de elasticidade do aço módulo de elasticidade reduzido do aço módulo de elasticidade do aço na camada $\mathrm{n}^{\circ} \mathrm{k}$ módulo de elasticidade do concreto na camada $\mathrm{n}^{\circ} \mathrm{k}$ módulo de elasticidade equivalente da camada $\mathrm{n}^{\circ} \mathrm{k}$ tensor constitutivo de rigidez elástica isótropo de quarta ordem para estados dominantes de tração tensor constitutivo de rigidez elástica isótropo de quarta ordem para estados dominantes de compressão força normal aplicada resistência à tração do concreto resistência à compressão do concreto função critério de início de dano em estados predominantes de tração

função critério de início de dano em estados predominantes de compressão módulo de elasticidade transversal do material danificado módulo de elasticidade transversal do material íntegro potencial anelástico em tração potencial anelástico em compressão hipersuperfície separadora dos domínios de deformação de tração e de compressão predominantes hipersuperfície separadora dos domínios de tensão de tração e de compressão predominantes tensor identidade de segunda ordem primeiro invariante do tensor de tensões tensor identidade de quarta ordem 


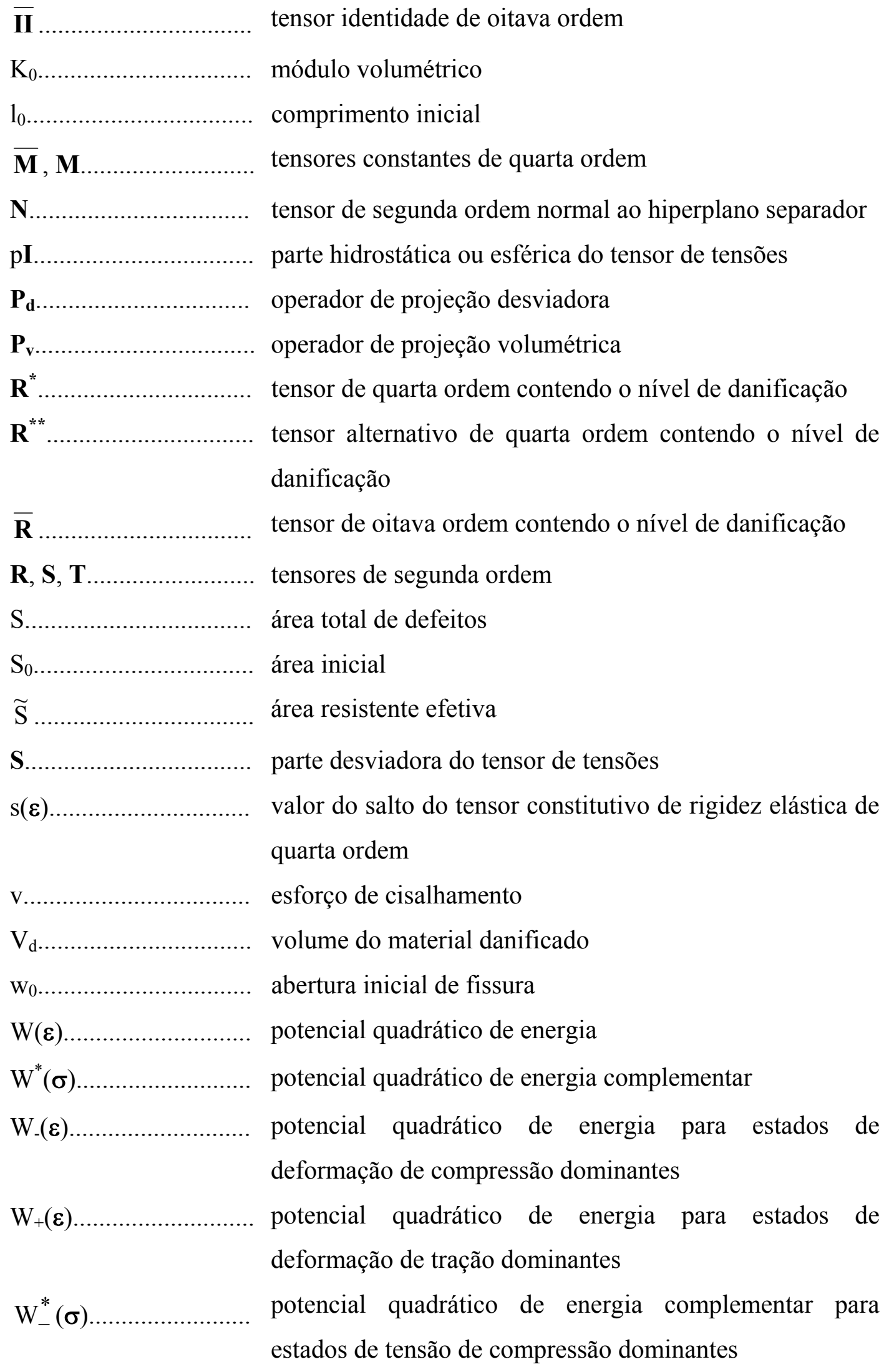




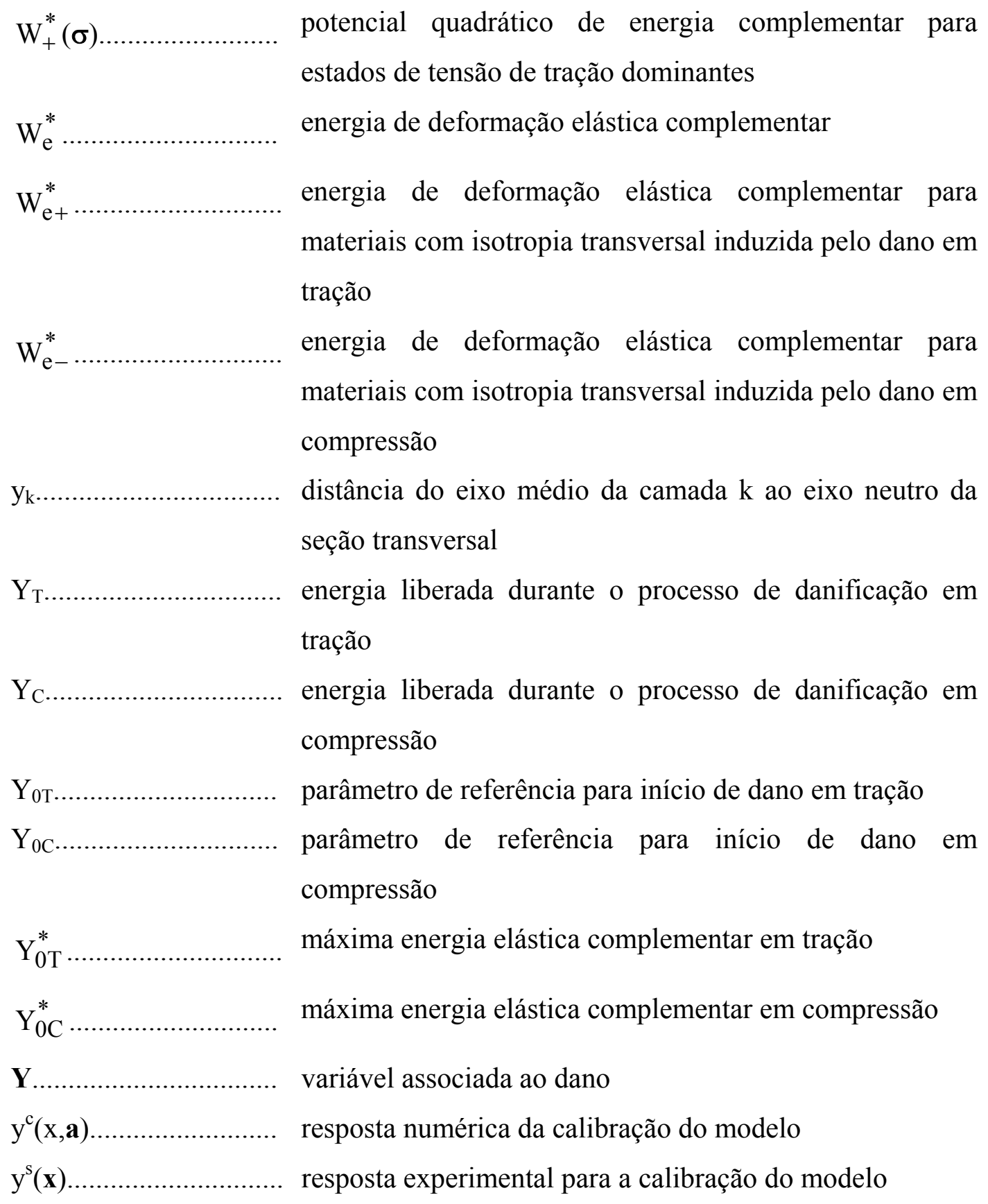




\section{RESUMO}

PITUBA, J. J. C. Sobre a Formulação de um Modelo de Dano para o Concreto. São Carlos, 151 p. Tese (Doutorado) - Escola de Engenharia de São Carlos, Universidade de São Paulo

Este trabalho trata da formulação de leis constitutivas para meios elásticos, que uma vez danificados passam a apresentar diferentes comportamentos em tração e em compressão e certo grau de anisotropia. Inicialmente é revista uma formulação para meios elásticos anisótropos e bimodulares, sendo a mesma, então, estendida para incorporar os casos de meios elásticos com anisotropia e bimodularidade induzidas pelo dano. Seguindo os conceitos da Mecânica do Dano Contínuo e a extensão mencionada da formulação, propõe-se um modelo constitutivo para o concreto assumindo por hipótese fundamental, a equivalência de energia entre meio danificado real e meio contínuo equivalente. Tal hipótese garante a simetria do tensor constitutivo e a sua consistência termodinâmica. De acordo com o modelo proposto, o material é considerado como um meio elástico inicialmente isótropo que passa a apresentar anisotropia induzida pela evolução do dano. Além disso, a danificação pode também induzir uma resposta bimodular no material, isto é, respostas elásticas diferentes para estados de tensão de tração ou de compressão predominantes. Nesse sentido, dois tensores de dano governando as rigidezes em regimes predominantes de tração ou de compressão são introduzidos. Sugere-se então, um critério afim de caracterizar os estados dominantes. As deformações permanentes induzidas pelo dano são, de uma forma geral, desconsideradas. No entanto, propõe-se uma versão unidimensional do modelo que permite a sua consideração. Por outro lado, os critérios para a ativação inicial dos processos de danificação e de sua posterior evolução são escritos em termos de densidade de energia de deformação. Os parâmetros do modelo podem ser identificados mediante experimentos, onde estados de tensão uniaxial e biaxial são induzidos. Também propõem-se leis de evolução de dano com base nos resultados experimentais. A boa coerência do modelo é ilustrada comparando-se uma série de respostas experimentais e numéricas no concreto relativas a estados de tensão uni, bi e triaxiais. Por fim, o modelo é empregado em análises unidimensionais e planas de vigas e pórtico em concreto armado com o objetivo de mostrar a sua potencialidade.

Palavras-chave: modelos constitutivos, concreto, mecânica do dano. 


\section{ABSTRACT}

PITUBA, J. J. C. On the formulation of Damage Model for the Concrete. São Carlos, 151 p. Tese (Doutorado) - Escola de Engenharia de São Carlos, Universidade de São Paulo

This thesis deals with the formulation of constitutive laws for elastic media that start to present different behaviours in tension and compression and some anisotropy degree when damaged. Initially a formulation for bimodular and anisotropic elastic media is reviewed; then it is extended to take into account the bimodularity and anisotropy induced by damage. Following the concepts of the Continuum Damage Mechanics and the formulation extension, a constitutive model is proposed here by exploring the fundamental hypothesis of energy equivalence between real and continuous medium. Such hypothesis guarantees symmetry to the constitutive tensor and its thermodynamic consistency. According to the proposed modeling, the material is assumed as an initial elastic isotropic medium presenting anisotropy induced by damage evolution. Moreover, damage can also induce a bimodular response in the material, i.e., distinct elastic responses whether traction or compression stress states prevail. To take into account the bimodularity conveniently, two damage tensors governing the rigidity in traction or compression regimes are introduced. A criterion is proposed in order to characterize the dominant states. In general, the permanent strains induced by damage are disregarded. However, a one-dimensional version of the model that allows considering permanent strains is proposed. On the other hand, damage criteria indicating the initial and further evolution of damage are expressed in terms of strain energy densities. The model parameters can be identified from experiments where one-axial and two-axial stress states are induced. Damage evolution laws are also proposed on the basis of experimental results. The good performance of the model is illustrated by comparing a variety of numerical and experimental responses, from one to three-axial stress states. Finally, the model is used in one-dimensional and plane analysis of reinforced concrete beams and frame in order to show its potentiality.

Keywords: constitutive models, concrete, damage mechanics. 


\section{INTRODUÇÃO}

\subsection{CONSIDERAÇÕES PRELIMINARES E SÍNTESE DO CONTEÚDO}

A contínua evolução tecnológica dos materiais, por um lado, e da formulação de modelos mecânicos combinada com técnicas computacionais, por outro lado, tem impulsionado a investigação das respostas não-lineares dos materiais e suas influências sobre o comportamento de estruturas. Este trabalho trata da formulação de modelos constitutivos para o concreto formulados pela Mecânica do Dano Contínuo, que se constitui numa ferramenta para a consideração da deterioração de materiais, devida exclusivamente ao processo de microfissuração distribuída. No caso do concreto, um material no qual a fissuração difusa em muitos casos é o fenômeno dominante no comportamento não-linear, a Mecânica do Dano é sem dúvida capaz de proporcionar modelos constitutivos apropriados. Nesse âmbito, a busca por modelos de dano cada vez mais realísticos justifica-se em função de diferentes fenômenos decorrentes da sua heterogeneidade.

Assim sendo, o objetivo final desta Tese é a proposição de um modelo constitutivo para o concreto, com as características de que permita levar em conta a anisotropia induzida pela danificação progressiva e que seja aplicável a situações mais complexas de carregamento. A formulação matemática adotada procura garantir a consistência com princípios da termodinâmica [PITUBA (1998)] e, também, pretende contornar alguns problemas evidenciados na formulação teórica de alguns modelos análogos propostos na literatura.

Entre os vários aspectos complementares a serem contemplados pelo modelo destacam-se: a consideração do caráter bimodular do comportamento mecânico do concreto e a isotropia transversal induzida pelo dano. 
De uma maneira geral, inserem-se neste trabalho estudos sobre o comportamento mecânico do concreto, a formulação de um modelo para meios elásticos anisótropos com comportamento bimodular e a sua extensão para os casos de materiais bimodulares danificados com anisotropia induzida pelo dano. Destacamse, em particular: a utilização de equivalência de energia para a obtenção de tensores constitutivos e de dano simétricos, a consideração de variáveis escalares de dano que são capazes de gerar a anisotropia material e um estudo para incorporar no modelo constitutivo proposto neste trabalho as deformações plásticas decorrentes do dano.

Com relação ao conteúdo específico do texto, a disposição de assuntos é descrita a seguir de forma resumida.

O capítulo 1, introdução, reúne um resumo sobre alguns conceitos da Mecânica do Dano no Contínuo, tais como: redução da ordem do tensor de dano e apresentação das equivalências de deformação, de tensão e de energia. Alguns aspectos sobre o comportamento físico do concreto são tratados em seguida, sendo discutidos desde os mecanismos básicos de microfissuração, observando-se a microestrutura do material, até o comportamento macroscópico do concreto sob solicitações uniaxiais e multiaxiais.

O capítulo 2 contempla a formulação de modelos constitutivos para meios elásticos danificados anisótropos com resposta bimodular. Inicialmente é apresentada a proposta de CURNIER, HE \& ZYSSET (1995) para meios elásticos anisótropos sem danificação com resposta bimodular. Em seguida, apresenta-se a proposição da extensão daquela formulação para meios danificados, com anisotropia induzida pelo dano. Com base nessa extensão, tensores constitutivos de alguns modelos de dano para o concreto são apresentados no final do capítulo.

No capítulo 3, apresenta-se a proposta de um modelo para o concreto baseada na formulação do capítulo anterior. A proposição pretende ser consistente com os princípios da termodinâmica, destacando-se os critérios para a ativação inicial e evolução do processo de danificação, além da caracterização dos comportamentos predominantes de tração e de compressão e de regras para a identificação do plano local de isotropia transversal do material. Apresenta-se, também, uma proposta da incorporação de deformações plásticas devido ao dano no modelo constitutivo gerado neste trabalho. 
No capítulo 4 discute-se a identificação dos parâmetros característicos do modelo, com destaque para o processo analítico (por mínimos quadrados) utilizado para a determinação da melhor aproximação numérica para os parâmetros. Em seguida, a influência dos parâmetros na curva tensão-deformação é apresentada. Finalmente, discutem-se a identificação dos parâmetros nos diversos ensaios experimentais contidos na literatura e as respostas fornecidas pelo modelo proposto.

No capítulo 5, reúnem-se resultados relativos à aplicação do modelo proposto neste trabalho, no estudo do comportamento de estruturas em concreto armado. Apresentam-se resultados de análises unidimensionais e planas, com o emprego de códigos de cálculo formulados com o Método dos Elementos Finitos. Comparam-se as respostas obtidas com o modelo com respostas experimentais e de outros modelos de dano para o concreto.

No sexto capítulo faz-se um relato das conclusões obtidas neste trabalho, além das perspectivas de ulteriores desenvolvimentos a partir deste.

No capítulo 7 apresentam-se as referências bibliográficas.

Finalmente, em apêndice, ilustram-se algumas relações matemáticas de interesse para a compreensão dos tensores e operações tensoriais envolvidos no trabalho.

\subsection{COMENTÁRIOS SOBRE A MECÂNICA DO DANO NO CONTÍNUO}

\subsubsection{Introdução}

Neste item comentam-se alguns aspectos da Mecânica do Dano de maior relevância para o trabalho.

Inicia-se a análise pela discussão da redução da ordem do tensor geral de dano, o qual permite relacionar os tensores constitutivos do material íntegro e do material danificado. Nessa discussão tem papel importante as equivalências de tensão, deformação e energia, entre meios íntegros e com dano, propostas na bibliografia. A possível redução de ordem do tensor geral de dano, além de diminuir o trabalho algébrico, permite estabelecer mais facilmente os conceitos de dano isótropo e anisótropo. 


\subsubsection{Tensor Geral de Dano e as Equivalências de Deformação, Tensão e Energia}

Dentro do escopo da Mecânica do Dano, os efeitos da danificação sobre as propriedades de um material inicialmente elástico se reproduzem no tensor constitutivo de rigidez. $\mathrm{O}$ dano se reflete numa redução de várias das componentes de rigidez, podendo o material danificado manter-se isótropo ou tornar-se anisótropo. Neste último caso, fatores de redução de rigidez associados a diferentes direções de simetria material devem ser identificados.

Limitando-se a abordagem ao caso de um meio elástico com dano, em qualquer estado de dano um tensor de quarta ordem $\mathbf{E}$, contendo módulos elásticos danificados, servirá para descrever a resposta do meio assim como o tensor de quarta ordem $\mathbf{E}_{\mathbf{0}}$ permite descrever a resposta elástica do material virgem. Para incluir também processos que não envolvem danificação e que preservam a rigidez inicial, o tensor $\mathbf{E}$ contém os módulos virgens afetados por fatores que incluem, em forma aditiva, as medidas do nível de dano, de modo que se essas medidas forem nulas recupera-se o tensor $\mathbf{E}_{\mathbf{0}}$.

Segundo CAUVIN (1997), a relação entre os dois tensores de quarta ordem $\mathbf{E}$ e $\mathbf{E}_{\mathbf{0}}$ pode ser expressa matematicamente segundo uma relação linear da seguinte forma:

$\mathbf{E}=\overline{\mathbf{R}} \quad \mathbf{E}_{0}$

O nível de danificação está contido no tensor de oitava ordem $\overline{\mathbf{R}}$. Quando não há dano, $\overline{\mathbf{R}}$ deve reduzir-se ao tensor identidade de oitava ordem $\overline{\mathbf{I I}}$ definido pela propriedade:

$\overline{\text { II }} \mathbf{E}_{\mathbf{0}}=\mathbf{E}_{0}$

Utilizando-se o tensor identidade, pode-se propor uma outra redação em forma aditiva para $\overline{\mathbf{R}}$, tal que o efeito da danificação aparece isolado num tensor de oitava ordem $\overline{\mathbf{D}}$. Segundo essa forma resultam:

$$
\overline{\mathbf{R}}=\overline{\mathbf{I I}}-\overline{\mathbf{D}} \quad \mathrm{e} \quad \mathbf{E}=(\overline{\mathbf{I}}-\overline{\mathbf{D}}) \quad \mathbf{E}_{0}
$$


Naturalmente, admitir-se que o processo físico de danificação possa ser representado por uma grandeza matemática de natureza tensorial constitui-se uma hipótese forte que deveria ser validada por observações experimentais.

Justifica-se essa representação tensorial admitindo-se que o dano possa ser quantificado por meio de uma variável associada ao ponto e segundo uma certa direção a partir desse ponto. Assim, genericamente a variável de dano pode assumir valores diferentes no ponto de acordo com a direção escolhida.

Uma hipótese complementar consiste em admitir continuidade para aquela variação; uma outra hipótese é que existam no espaço três direções ortogonais de danificação principal. Segue daí o caráter tensorial.

Obviamente que para garantir a existência de auto-valores reais de dano, a simetria do tensor é algo desejável.

Sendo então válida a (1.3), ainda assim, para fins práticos, a utilização de um tensor de dano de oitava ordem com seus $3^{8}$ elementos é algo inviável. Como será visto mais adiante, simetrias materiais e os princípios de equivalências de deformações, de tensões e de energia serão invocados como forma de reduzir a ordem do tensor de dano e melhorar sua operacionalidade.

\section{Princípios gerais de equivalências: análise unidimensional}

Considere-se um corpo de comprimento inicial $1_{0}$ e seção transversal com área $\mathrm{S}_{0}$. Admite-se que tal corpo passe a ser submetido a uma força de tração uniaxial. Por efeito da força aplicada $\mathrm{F}$ o corpo sofre um alongamento $\delta$, e com as hipóteses de meio íntegro e resposta homogênea em todos os pontos do corpo, a tensão e deformação nominais são definidas como: $\sigma=\frac{F}{S_{0}}$ e $\varepsilon=\frac{\delta}{1_{0}}$. Todavia o meio pode apresentar previamente microfissuras, o que de fato é mais consistente com a realidade. Pode-se então assumir que a abertura das microfissuras computadas de forma equivalente contribuem para uma parcela representada por $\Delta \mathrm{d}$ da variação do comprimento inicial. Por outro lado, segundo uma certa seção de referência, as microfissuras implicam numa redução da área que efetivamente transmite o esforço solicitante. Por consequência, a área da seção efetivamente resistente será uma parte 
da área inicial: $\widetilde{\mathrm{S}}=\alpha \mathrm{S}_{0},(0<\alpha<1)$ e somente a parcela $(\delta-\Delta \mathrm{d})$ do alongamento total é que efetivamente deve ser considerada na medida de deformação linear. Resultam daí os conceitos de esforço e deformação efetivos (Figura 1.1a):

$\widetilde{\sigma}=\frac{\mathrm{F}}{\widetilde{\mathrm{S}}} \quad$ e $\quad \widetilde{\varepsilon}=\frac{\delta-\Delta \mathrm{d}}{1_{0}}$
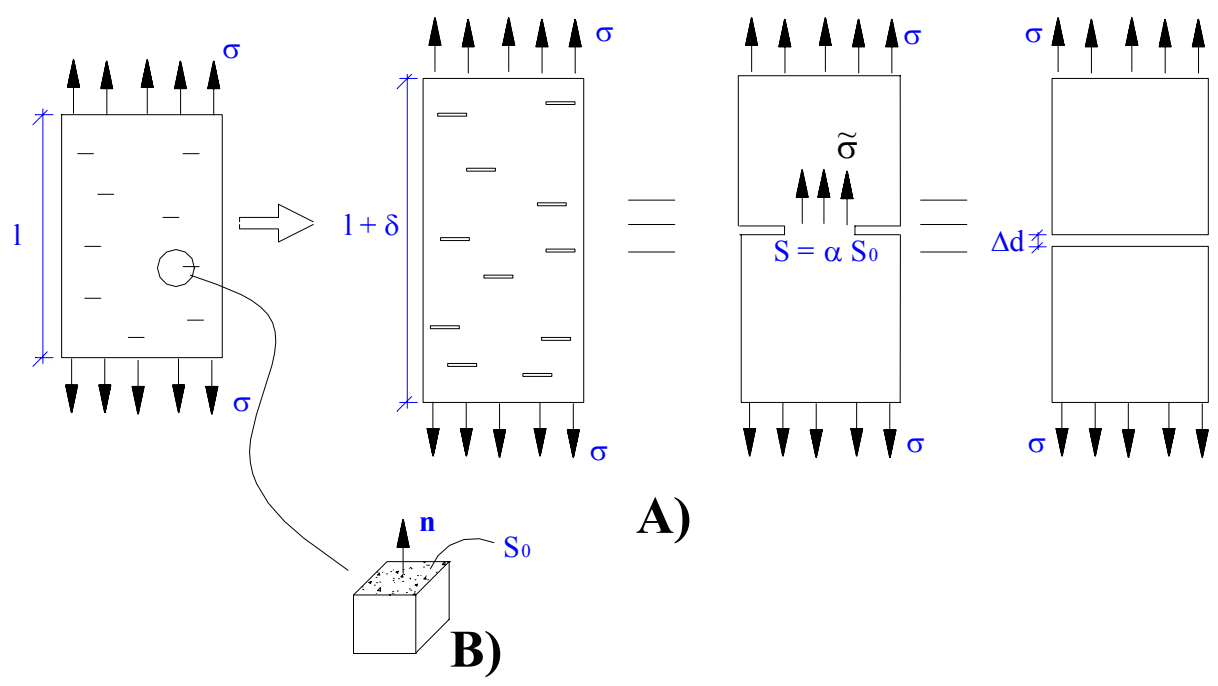

A)

Figura 1.1 - a) Representação simbólica de esforço e deformação nominais e efetivas no caso uniaxial. b) Elemento de volume representativo do corpo sob tração uniaxial.

Uma primeira definição possível para a variável de dano é representada na relação seguinte [LEMAITRE \& CHABOCHE (1990)]:

$\mathrm{D}=\frac{\mathrm{S}}{\mathrm{S}_{0}}$

onde $\mathrm{S}=\mathrm{S}_{0}-\widetilde{\mathrm{S}}$ define a área dos defeitos medidos e $\mathrm{D}$ representa uma medida local do dano. A variável de dano assume valores contidos no intervalo $0 \leq \mathrm{D} \leq 1$, sendo que $\mathrm{D}=0$ tem correspondência com a situação de material íntegro e $\mathrm{D}=1$ indica um estado de total deterioração.

A definição em questão pode ser associada a um ponto e pressupõe uma distribuição uniforme dos microdefeitos segundo qualquer direção a partir do ponto.

Imaginando-se uma situação em que as microfissuras se distribuíssem segundo uma direção privilegiada, a variável $\mathrm{D}$ poderia assumir valores diferentes de acordo com a direção de observação a partir do ponto. 
Com a (1.5), a parcela da seção que efetivamente resiste ao esforço aplicado pode ser escrita em função da variável de dano como:

$\widetilde{\mathrm{S}}=\mathrm{S}_{0}-\mathrm{S}=\mathrm{S}_{0}(1-\mathrm{D})$

Da expressão (1.4a), a tensão efetiva resulta em:

$\widetilde{\sigma}=\frac{F}{\widetilde{S}}=\frac{F}{S_{0}(1-D)} \quad \therefore \quad \widetilde{\sigma}=\frac{\sigma}{(1-D)}$

Obviamente $\tilde{\sigma} \geq \sigma$ e em particular

$\widetilde{\sigma}=\sigma \quad$ para material localmente íntegro

$\tilde{\sigma} \rightarrow \infty \quad$ para material totalmente danificado localmente

Uma segunda definição para uma variável de dano pode ser proposta levandose em conta a relação entre a parcela de alongamento por abertura de defeitos e o próprio alongamento.

$\mathrm{D}^{*}=\frac{\Delta \mathrm{d}}{\delta}$

Com essa nova variável e levando-se em conta a expressão (1.4b), tem-se como medida efetiva da deformação linear

$\widetilde{\varepsilon}=\left(1-D^{*}\right) \varepsilon$

Considerando-se que o volume $\mathrm{V}_{\mathrm{d}}$, correspondente ao material danificado envolvido nas definições de tensão e de deformação efetiva é o mesmo, tem-se que:

$\mathrm{V}_{\mathrm{d}}=\mathrm{S} \cdot \delta=\mathrm{S}_{0} \cdot \Delta_{\mathrm{d}}$

Segue daí a igualdade entre as variáveis de dano (1.5) e (1.8), D = D* .

As quantidades efetivas são portanto relativas à parte íntegra do meio e relacionam-se pela rigidez elástica na forma:

$\widetilde{\sigma}=\mathrm{E}_{0} \widetilde{\varepsilon}$ 
A introdução das relações (1.7) e (1.9) na (1.11) permite escrever a lei constitutiva em termos de quantidades nominais, que são normalmente utilizadas na modelagem com a hipótese do meio contínuo. A substituição daquelas relações pode, entretanto, ser guiada por hipóteses sobre as equivalências de deformação, de tensão ou de energia.

A hipótese de equivalência de deformação, proposta por LEMAITRE \& CHABOCHE (1990), é expressa nos seguintes termos:

"O estado de deformação de um material com dano é obtido da lei de comportamento do material íntegro onde a tensão nominal é substituída pela tensão efetiva." (Figura 1.2)

Assume-se portanto, uma igualdade entre deformações nominal e efetiva:

$$
\varepsilon=\widetilde{\varepsilon}
$$

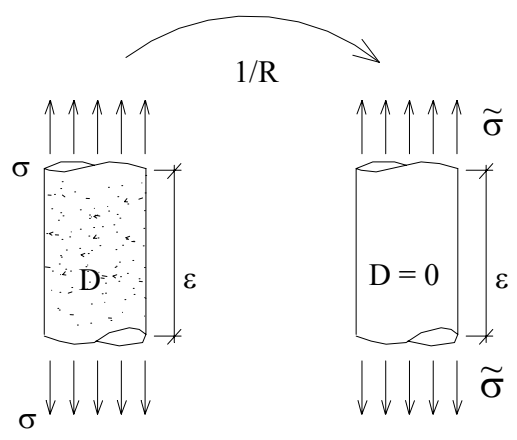

Figura 1.2 - Hipótese de deformação equivalente.

Valendo o princípio, a relação (1.11) passa a assumir a seguinte forma:

$\widetilde{\sigma}=\mathrm{E}_{0} \varepsilon \quad \Rightarrow \quad \frac{\sigma}{(1-\mathrm{D})}=\mathrm{E}_{0} \varepsilon \therefore \sigma=(1-\mathrm{D}) \mathrm{E}_{0} \varepsilon$

Da expressão acima obtém-se uma relação que define o módulo secante de rigidez elástica $\mathrm{E}$ para um meio contínuo de resposta equivalente ao meio deteriorado $\mathrm{E}=(1-\mathrm{D}) \mathrm{E}_{0}$

Essa última relação coloca em evidência a degradação das características mecânicas do material causadas pelo dano, e dela resulta, também, uma outra relação para a identificação da variável de dano: 
$\mathrm{D}=1-\frac{\mathrm{E}}{\mathrm{E}_{0}}$

Como uma alternativa para a hipótese de equivalência em deformação, podese considerar a seguinte hipótese dual de equivalência de tensão:

"O estado de tensão associado a um meio danificado é obtido da lei de comportamento do material íntegro onde a deformação nominal é substituída pela deformação efetiva.” (Fig. 1.3)

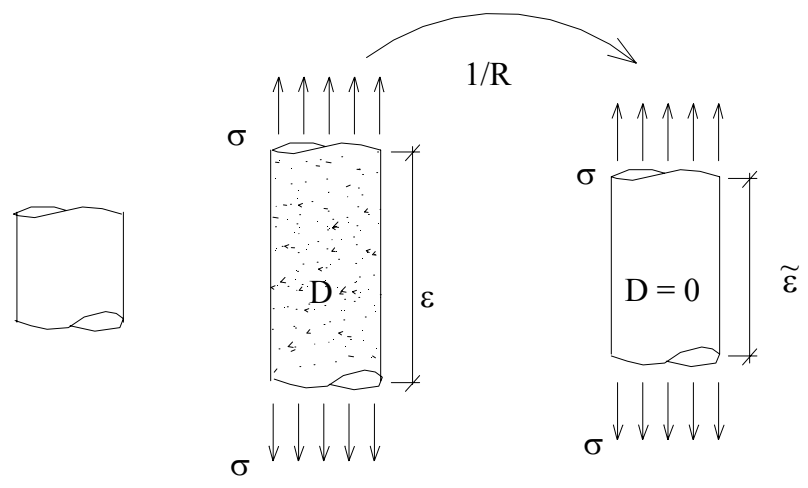

Figura 1.3 - Hipótese de tensão equivalente.

Assume-se portanto, uma igualdade entre tensões nominal e efetiva:

$\sigma=\tilde{\sigma}$

Com a nova hipótese de equivalência a expressão (1.11) passa a ser escrita da seguinte forma:

$\sigma=\mathrm{E}_{0} \widetilde{\varepsilon} \quad \Rightarrow \quad \sigma=\mathrm{E}_{0} \varepsilon(1-\mathrm{D}) \therefore \sigma=(1-\mathrm{D}) \mathrm{E}_{0} \varepsilon$

A variável de dano pode ser identificada por uma relação idêntica à (1.15).

Uma última alternativa de equivalência de respostas constitutivas analisada neste trabalho é a equivalência de energia elástica, a qual postula equivalência entre a energia envolvida na parcela de meio danificado e na parcela de meio íntegro.

$\widetilde{\sigma \varepsilon}=\sigma \varepsilon$

Esse princípio é diretamente verificado, substituindo-se na relação (1.11) as definições da tensão e deformação efetivas: 
$\widetilde{\sigma}=\mathrm{E}_{0} \widetilde{\varepsilon} \quad \Rightarrow \quad \frac{\sigma}{(1-\mathrm{D})}=\mathrm{E}_{0} \varepsilon(1-\mathrm{D}) \therefore \sigma=(1-\mathrm{D})^{2} \mathrm{E}_{0} \varepsilon$

Da expressão acima pode-se definir uma relação para o módulo secante de rigidez elástica $\mathrm{E}$ para um meio contínuo de resposta equivalente ao meio deteriorado $\mathrm{E}=(1-\mathrm{D})^{2} \mathrm{E}_{0}$

colocando-se em evidência a degradação das características mecânicas do material causadas pelo dano. Nesse caso, a variável de dano pode ser identificada por:

$\mathrm{D}=1-\left(\frac{\mathrm{E}}{\mathrm{E}_{0}}\right)^{\frac{1}{2}}$

\section{Princípios gerais de equivalência: análise multiaxial}

As hipóteses de equivalência apresentadas e suas conseqüências sobre relações constitutivas resultantes no caso uniaxial, podem ser generalizadas para casos multiaxiais, permitindo relacionar tensores de segunda ordem de tensão e de deformação por meio de um tensor constitutivo de rigidez secante elástica com dano de quarta ordem. A propriedade de simetria do tensor constitutivo depende da equivalência adotada, como se verá mais adiante.

Inicialmente, com a mesma forma da relação (1.11) escreve-se uma nova relação geral envolvendo agora tensores de segunda ordem efetivos:

$\widetilde{\sigma}=\mathbf{E}_{0} \widetilde{\varepsilon}$

Uma questão importante é sobre a definição desses tensores efetivos, e ela será abordada no que segue em função do estudo da redução de ordem do tensor geral de dano.

\section{$\underline{\text { Redução da ordem do tensor geral de dano }}$}

O objetivo é mostrar que é possível escrever uma lei constitutiva para um material danificado através de tensores de dano de quarta ordem. 
Observando-se inicialmente a relação (1.22) entre os tensores de tensão e de deformação efetivas e aplicando-se o princípio de equivalência de deformações $\widetilde{\varepsilon}=\varepsilon$, obtém-se:

$\tilde{\sigma}=\mathbf{E}_{0} \varepsilon \quad \therefore \quad \varepsilon=\mathbf{E}_{0}^{-1} \tilde{\sigma}$

Considerando-se a lei constitutiva secante para o meio danificado,

$\sigma=\mathbf{E} \varepsilon$

e com a expressão (1.23), tem-se que:

$\sigma=\mathbf{E E}_{0}^{-1} \tilde{\sigma} \quad \therefore \quad \sigma=\mathbf{R}^{*} \tilde{\sigma}$

onde $\mathbf{R}^{*}$ é um tensor de quarta ordem definido como:

$\mathbf{R}^{*}=\mathbf{E E}_{0}^{-1}$

Valendo, portanto, a equivalência de deformação, da (1.26) segue que

$\mathbf{E}=\mathbf{R}^{*} \mathbf{E}_{0}$

Conclui-se que não há a necessidade de um tensor de oitava ordem para relacionar $\mathbf{E}$ e $\mathbf{E}_{\mathbf{0}}$.

Por outro lado, levando-se em conta a expressão que define o tensor geral de oitava ordem (1.3), a (1.26) resulta:

$\mathbf{R}^{*}=\overline{\mathbf{R}} \mathbf{E}_{0} \mathbf{E}_{0}^{-1} \quad \therefore \quad \mathbf{R}^{*}=\overline{\mathbf{R}} \mathbf{I I}$

onde II é o tensor identidade de quarta ordem.

É possível se chegar na mesma conclusão anterior adotando-se a equivalência de tensão.

De fato, levando-se em conta que nesta equivalência postula-se que $\tilde{\sigma}=\sigma$, e ainda as expressões (1.22) e (1.24), obtém-se:

$\mathbf{E}_{0} \widetilde{\varepsilon}=\mathbf{E} \varepsilon \quad \therefore \quad \widetilde{\varepsilon}=\mathbf{E}_{0}^{-1} \mathbf{E} \varepsilon \quad \therefore \quad \widetilde{\varepsilon}=\mathbf{R}^{* *} \varepsilon$

onde $\mathbf{R}^{* *}$ é um tensor de quarta ordem definido como: 
$\mathbf{R}^{* *}=\mathbf{E}_{0}^{-1} \mathbf{E}$

Portanto, valendo a equivalência de tensão, segue que

$\mathbf{E}=\mathbf{E}_{0} \mathbf{R}^{* *}$

Novamente conclui-se que não há necessidade de um tensor de oitava ordem para relacionar $\mathbf{E}$ e $\mathbf{E}_{\mathbf{0}}$.

Por outro lado, com a expressão do tensor geral de dano de oitava ordem (1.3) na (1.30), resulta que:

$\mathbf{R}^{* *}=\mathbf{E}_{0}^{-1} \overline{\mathbf{R}} \mathbf{E}_{0} \quad \therefore \quad \mathbf{R}^{* *}=\overline{\mathbf{R}} \mathbf{I I}$

Outro ponto importante diz respeito à simetria do tensor de rigidez secante elástica do meio danificado, que só é garantida com a adoção da equivalência de energia.

Levando-se em conta a equivalência de energia, $\widetilde{\sigma} \cdot \widetilde{\varepsilon}=\sigma \cdot \varepsilon$, e as expressões (1.25) e (1.29) de tensão e deformação efetivas, respectivamente, obtém-se como resultado:

$$
\begin{array}{lllll}
\tilde{\sigma} \cdot \widetilde{\varepsilon} & =\sigma \cdot \varepsilon \therefore & & \mathbf{R}^{*^{-1}} \sigma \cdot \mathbf{R}^{* *} \varepsilon=\sigma \cdot \varepsilon \therefore & \mathbf{R}^{* *^{\mathrm{T}}} \mathbf{R}^{*^{-1}} \sigma \cdot \varepsilon=\sigma \cdot \varepsilon \therefore \mathbf{R}^{* *^{\mathrm{T}}} \mathbf{R}^{*^{-1}}=\mathbf{I I} \\
\mathbf{R}^{* *^{\mathrm{T}}}=\mathbf{R}^{*} & \text { ou } & \mathbf{R}^{*^{-1}}=\mathbf{R}^{* *^{-\mathrm{T}}} &
\end{array}
$$

Com as definições de $\mathbf{R}^{*}$ e $\mathbf{R}^{* *}$ dadas pelas (1.26) e (1.30) e com a hipótese de $\mathbf{E}_{\mathbf{0}}=\mathbf{E}_{0}^{\mathbf{T}}$, a (1.33) fornece $\mathbf{E}=\mathbf{E}^{\mathbf{T}}$. Portanto a equivalência de energia implica em simetria do tensor de rigidez secante do meio danificado.

Voltando à questão da definição dos tensores efetivos de tensão e de deformação, a forma unidimensional do tensor de tensão efetivo sugere para o caso multiaxial a seguinte definição para o tensor $\mathbf{R}^{*}$ :

$$
\mathbf{R}^{*}=(\mathbf{I I}-\mathbf{D})
$$

Nesse caso os tensores identidade (II) e de dano (D) são de quarta ordem.

Conseqüentemente para a tensão efetiva passa a valer a relação:

$$
\widetilde{\sigma}=(\mathbf{I I}-\mathbf{D})^{-1} \boldsymbol{\sigma}
$$


Impondo-se equivalência de deformação, $\widetilde{\varepsilon}=\varepsilon$, e a (1.35), a relação geral (1.22) resulta em:

$\sigma=(\mathbf{I I}-\mathbf{D}) \mathbf{E}_{0} \varepsilon$

Nesse caso, o tensor de rigidez secante elástica do meio danificado fica dado por:

$\mathbf{E}=(\mathbf{I I}-\mathbf{D}) \mathbf{E}_{0}$

apresentando apenas a simetria menor, pois permite relacionar tensores simétricos de tensão e de deformação. forma:

Por outro lado, de forma independente, pode-se adotar para o tensor $\mathbf{R}^{* *}$ a

$\mathbf{R}^{* *}=(\mathbf{I I}-\mathbf{D})^{\mathbf{T}}$

Consequentemente para a deformação efetiva passa a valer a relação:

$\widetilde{\varepsilon}=(\mathbf{I I}-\mathbf{D})^{\mathbf{T}} \varepsilon$

Impondo-se a equivalência de tensão, $\widetilde{\sigma}=\sigma$, e a (1.39), a relação geral (1.22) passa a ser escrita da seguinte forma:

$\sigma=\mathbf{E}_{0}(\mathbf{I I}-\mathbf{D})^{\mathbf{T}} \varepsilon$

Dessa forma obtém-se o tensor de rigidez secante elástica do meio danificado,

$\mathbf{E}=\mathbf{E}_{0}(\mathbf{I I}-\mathbf{D})^{\mathbf{T}}$

que também apresenta apenas a simetria menor.

Finalmente, fazendo-se uso das definições adotadas para $\mathbf{R}^{*}$ e $\mathbf{R}^{* *}$ na relação geral (1.22) obtém-se:

$$
\begin{aligned}
& (\mathbf{I I}-\mathbf{D})^{-1} \boldsymbol{\sigma}=\mathbf{E}_{0}(\mathbf{I I}-\mathbf{D})^{\mathbf{T}} \boldsymbol{\varepsilon} \\
& \sigma=(\mathbf{I I}-\mathbf{D}) \mathbf{E}_{0}(\mathbf{I I}-\mathbf{D})^{\mathbf{T}} \varepsilon
\end{aligned}
$$

O tensor de rigidez secante elástica do meio danificado fica então escrito na forma: 


$$
\mathbf{E}=(\mathbf{I I}-\mathbf{D}) \mathbf{E}_{0}(\mathbf{I I}-\mathbf{D})^{\mathbf{T}}
$$

Observa-se que este tensor é simétrico (com simetrias menor e maior).

Nota-se, finalmente, que as formas gerais que permitem contemplar a anisotropia induzida pela danificação podem ser consideradas, como se verá mais adiante, na definição do tensor de dano de quarta ordem. Neste trabalho são tratados exclusivamente os modelos que envolvem variáveis de dano escalar.

\subsection{COMPORTAMENTO MECÂNICO DO CONCRETO}

\subsubsection{Introdução}

Neste item são abordadas algumas características do comportamento mecânico do concreto quando sujeito a diferentes tipos de solicitações.

Inicialmente descrevem-se os mecanismos básicos de micro-fissuração, ou dano do concreto, observando-se a sua microestrutura. Tais mecanismos ocorrem na média-escala e são discutidos com vistas à justificação das respostas macromecânicas.

Em seguida, à luz dos mecanismos descritos, apresenta-se uma análise do comportamento macroscópico do concreto sob solicitações uniaxiais de compressão e de tração, assim como sob solicitações multiaxiais. Pretende-se com isso, justificar as hipóteses utilizadas pelo modelo proposto neste trabalho, de simular o comportamento do concreto como inicialmente isótropo e com isotropia transversal induzida pelo dano.

\subsubsection{Mecanismos de Danificação do Concreto}

Do ponto de vista da média-escala, o concreto pode ser tratado como um material bifásico composto por uma fase agregado e uma fase matriz de cimento ou argamassa, por sua vez constituída de partículas de agregados finos e cimento como aglomerante. A heterogeneidade da estrutura da argamassa e as variações 
volumétricas que acontecem em decorrência do processo de cura, criam no material degradações iniciais (dano) de natureza irreversível.

De uma maneira geral o dano inicial é constituído por defeitos na zona de interface entre as fases e por vazios ou poros na argamassa.

A existência, por um lado, de uma fase estável (os agregados) e, por outro lado, de uma fase evolutiva do ponto de vista constitutivo (a pasta sofre retração e expansão na fase de cura, quando a resistência ainda é fraca) conduz à caracterização de uma zona de fraca resistência ao redor dos grãos, chamada "zona de transição". $\mathrm{Na}$ zona de transição formam-se defeitos de aderência e vazios quase sempre associados à direção de moldagem (Figura 1.4).

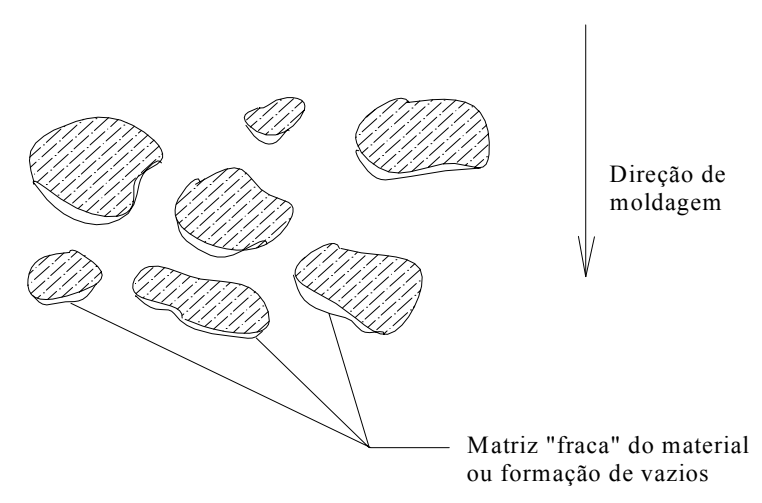

Figura 1.4 - Defeitos sob partículas de agregados [VAN MIER (1984)].

O processo de evolução do dano, ou danificação, difere de acordo com o tipo de solicitação. Os principais mecanismos de danificação, comuns a todos os estados de solicitação, podem ser caracterizados observando-se na média escala a resposta do material nos estados uniaxiais de tração e de compressão.

Admitindo-se, por simplificação, que os agregados tenham uma forma circular, no concreto sob tração a evolução da fissuração se produz em duas etapas (Figura 1.5):

- de início, o desenvolvimento se dá em modo misto de abertura e escorregamento (I e II, respectivamente) ao longo de um hemisfério dos grãos; isto pode acontecer no hemisfério inferior ou no superior, de acordo com a distribuição dos defeitos iniciais;

- em seguida, completado o hemisfério, passa a ocorrer a propagação instável em modo I culminando numa fratura perpendicular à direção do carregamento. 


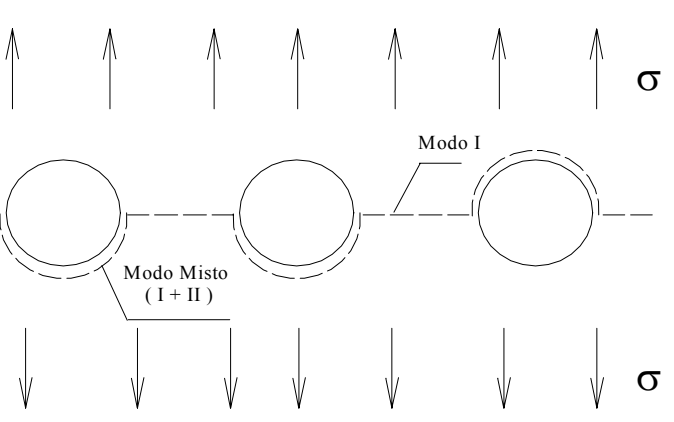

Figura 1.5 - Danificação em tração com início na zona de interface [MAZARS (1984)].

Entende-se que existem três tipos básicos de modos de abertura de fissura. São eles:

- Modo I: a abertura de fissura é devido a um esforço perpendicular à superfície do defeito.

- Modo II: há escorregamento das superfícies da fissura devido a um esforço de cisalhamento aplicado no plano dessas superfícies e paralelo ao defeito.

- Modo III: há escorregamento das superfícies da fissura, devido a um esforço cisalhante atuante no plano dessas superfícies e em direção perpendicular ao defeito.

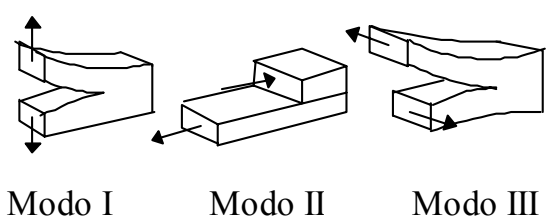

Figura 1.6 - Modos de abertura.

O processo de fissuração em tração uniaxial rende ao concreto um comportamento de isotropia transversal, já que numa direção (paralela ao carregamento) o material tem um comportamento diferente das outras duas direções (Figura 1.7).

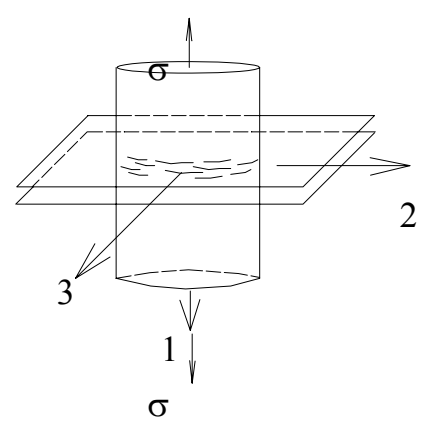

Figura 1.7 - Comportamento de isotropia transversal em tração uniaxial no concreto. 
Já a compressão uniaxial a danificação tem as características indicadas na figura 1.8. A primeira fissura aparece com modo II de abertura na região do contorno do agregado, aproximadamente à $30^{\circ}$ em relação a horizontal. A fissura progride ao longo da interface passando a apresentar um modo I de abertura. Em seguida, sua evolução se dá na direção da solicitação, deixando a região de interface para se propagar na matriz. Para elevados níveis de solicitação evidencia-se um panorama de fissuras paralelas à direção de carregamento (BUYUKOZTURK, NILSON \& SLATE (1971)).

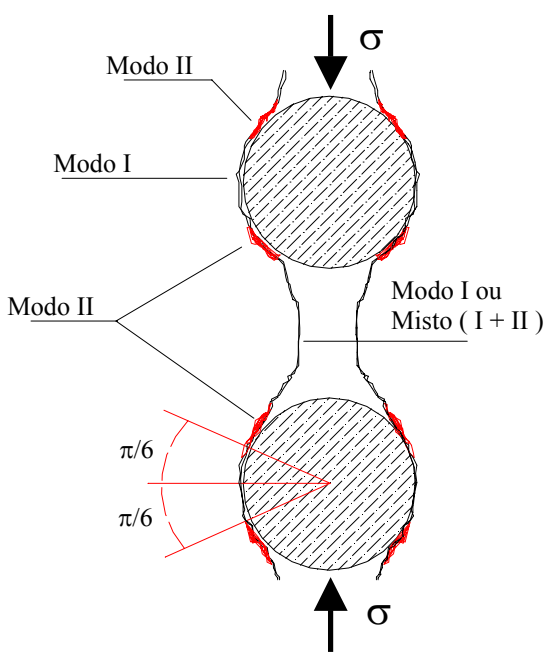

Figura 1.8 - Danificação em compressão com início na zona de interface [BUYUKOZTURK, NILSON \& SLATE (1971)].

O comportamento de material isótropo transversal pode também ser admitido para o concreto em casos de compressão uniaxial. Mais uma vez a direção paralela ao carregamento tem um comportamento diferente das outras duas direções.

\subsubsection{Comportamento Macroscópico do Concreto em Compressão Uniaxial}

Em ensaios de compressão uniaxial com deformação controlada [VAN MIER (1984)] nota-se que a partir do nível de carga máxima a relação tensão-deformação do concreto passa a mostrar um gradual decréscimo de resistência com o aumento da deformação axial. Este é o chamado comportamento "softening", que pode ser traduzido como amolecimento ou abrandamento. Em termos de fissuração, observase que macrofissuras se formam após o nível de tensão de pico. 
Normalmente espera-se que as macrofissuras se desenvolvam paralelas à direção da solicitação de compressão. Entretanto, particularmente próximo das regiões de contorno podem se desenvolver fissuras inclinadas e distribuídas aleatoriamente com várias orientações (DIAZ \& HILSDORF (1973)). A explicação mais comum sobre esta questão é que as fissuras inclinadas decorrem do efeito de confinamento provocado pelo atrito entre o sistema de aplicação de carga e o espécime. Essa vinculação, embora reduzida consideravelmente, também está presente em testes com condições de contorno com pouco atrito. As tensões de cisalhamento induzidas na interface sistema de aplicação de carregamento e espécime têm considerável influência sobre o valor da resistência de pico e sobre a curvatura do ramo de amolecimento da curva tensão-deformação uniaxial.

Alguns trabalhos foram realizados para estudar o comportamento do concreto sujeito a uma carga uniaxial de compressão. Dentre eles pode-se citar: HSU et al. $(1963)^{1}$ apud VAN MIER (1984), que realizaram um trabalho pioneiro na correspondência entre propagação de microfissuras e comportamento tensãodeformação do concreto, observando mudanças no comportamento macroscópico em cilindros de concreto que foram acompanhadas por variações na densidade de fissuras e modo de fissuração; DHIR \& SANGHA (1974)² apud MAZARS (1984) por sua vez, utilizaram no seu trabalho uma técnica com raio-X em microscópios para amostras em microconcreto; BENOUNICHE. (1979)³ apud MAZARS (1984), que mediram o tempo de percurso de ondas ultra-sônicas para atravessar um cilindro de concreto na direção da base.

Constata-se pelo conjunto de ensaios, a boa correspondência entre as diferentes fases obtidas por cada tipo de observação. Essas fases de comportamento do material podem ser definidas com base no diagrama tensão-deformação em compressão uniaxial e relacionadas com o processo de danificação da média-escala (Figura 1.9):

\footnotetext{
${ }^{1} \mathrm{HSU}$ et al.., T. T. C. . Microcracking of plain concrete and the shape of stress-strain curva. ACI Journal, February 1963, pp. 209-224.

${ }^{2}$ DHIR, R. H. e SANGHA, M. . Development and propagation of microcracks in plain concrete. Materiaux et Constructions, n. 37, 1974, pp. 17-23.

${ }^{3}$ BENOUNICHE, S. . Modélisation de l'endommagement du béton hydraulique par microfissuration en compression, Thèse de 3ème cycle, Paris 6, 1979.
} 
- uma fase quase-elástica se desenvolve desde o estado inicial até 30 - 40\% da resistência à compressão $\left(f_{c}\right)$. Tal fase é caracterizada por uma certa manutenção da rigidez e do coeficiente de Poisson iniciais do material. Na média-escala não ocorrem alterações importantes na configuração das microfissuras iniciais. O leve desvio de linearidade observado deve-se ao início de escorregamentos entre grãos e argamassa;

- uma fase não-linear estável é caracterizada a partir de um nível em torno de $40-50 \%$ de $\mathrm{f}_{\mathrm{c}}$, estendendo-se até $70-80 \%$ de $\mathrm{f}_{\mathrm{c}}$. Nessa fase a rigidez diminui sensivelmente porém o coeficiente de Poisson permanece inalterado. Além disso, a componente de deformação plástica longitudinal cresce lentamente e a sua componente transversal permanece praticamente nula. Na média-escala ocorre uma danificação evolutiva na interface agregado-argamassa de cimento e areia;

- acima de 70 - 80\% de $f_{c}$ até o valor de pico, caracteriza-se uma fase com coeficiente de Poisson crescente e deformação plástica evolutiva. Na média-escala há uma forte interação entre as fissuras de interface, resultando nas fissuras visíveis;

- além da tensão de pico, há a formação do ramo descendente da curva resposta até que seja atingida a deformação última do material, onde se dá a ruptura por esmagamento. Nessa última fase, ocorrem fortes alterações na média-escala pela evolução e integração das fisssuras na argamassa.

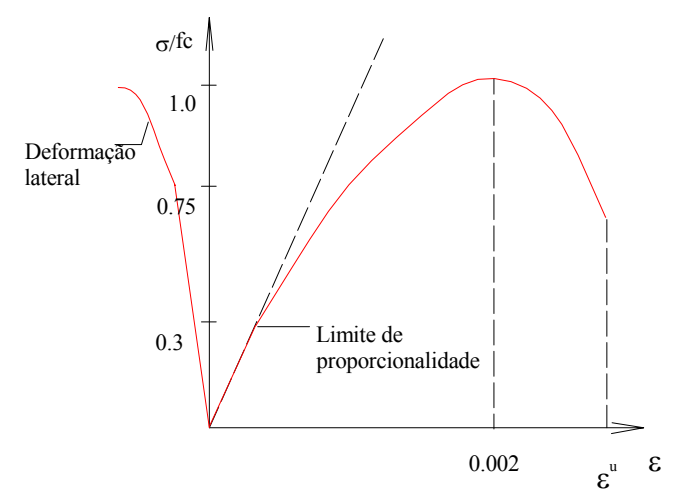

Figura 1.9 - Diagrama tensão-deformação em compressão [PROENÇA (1988)].

A evolução da danificação nas fases não-lineares rende ao material anisotropia (isotropia transversal), evidenciada pela orientação privilegiada da microfissuração na direção paralela ao carregamento, onde o plano 2-3 é o plano de isotropia transversal. 


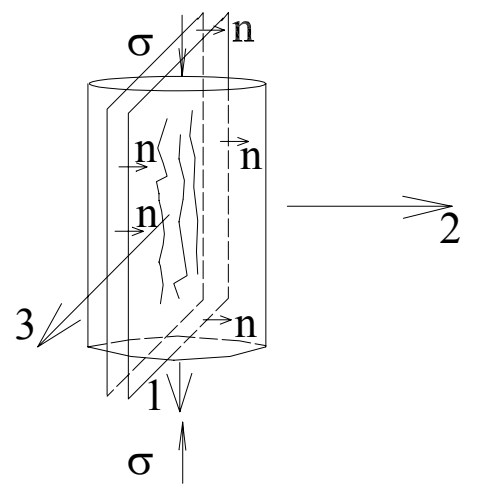

Figura 1.10 - Comportamento de isotropia transversal em compressão uniaxial no concreto.

Dois outros aspectos da resposta macroscópica merecem destaque: a variação volumétrica e a mudança do coeficiente de Poisson.

Quanto à deformação volumétrica, no trabalho de STROEVEN (1979), mostra-se que existe um nível de tensão de compressão a partir do qual ocorre uma reversão no diagrama deformação volumétrica versus tensão (Figura 1.11). Na média-escala a superfície de fissura por unidade de volume passa a aumentar mais rapidamente na etapa de reversão da deformação volumétrica.

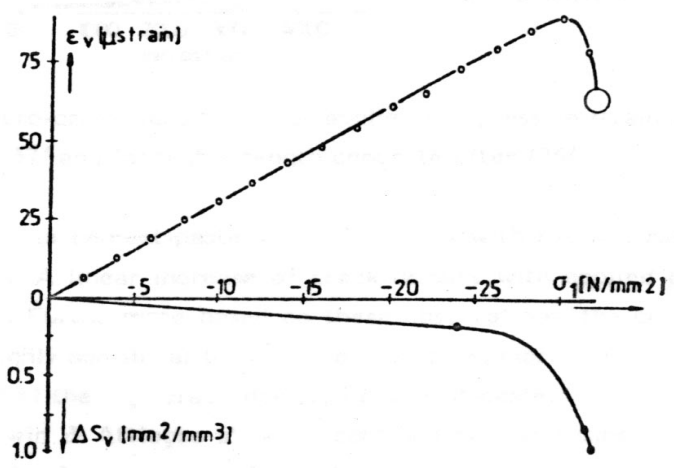

Figura 1.11 - Deformação Volumétrica $\left(\varepsilon_{\mathrm{v}}=\left(\varepsilon_{1}+\varepsilon_{2}+\varepsilon_{3}\right) / 3\right)$ e área específica de superfície de fissura versus tensão axial aplicada [STROEVEN (1979)].

Por outro lado, um valor típico para o coeficiente de Poisson nos concretos usuais é 0,2 ; entretanto esse valor cresce tendendo a 0,5 ao se aproximar da tensão de pico e na fase de abrandamento em conseqüência do aumento da área superficial de fissuras (Figura 1.12). 


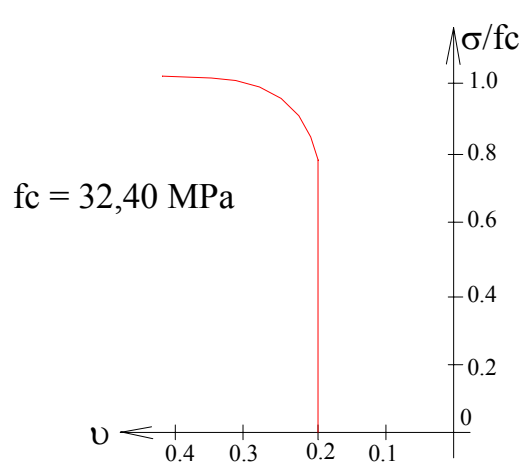

Figura 1.12 - Variação do coeficiente de Poisson [PROENÇA (1988)].

\subsubsection{Comportamento Macroscópico do Concreto em Tração Uniaxial}

O ensaio de tração direta é de difícil realização, e mesmo a interpretação de resultados de ensaios alternativos de flexão e de fendilhamento fica prejudicada por dois problemas:

- a instabilidade do comportamento pós-pico;

- os efeitos de escala e de altos gradientes locais de tensões.

Além dos problemas citados, que passarão a ser brevemente abordados neste item, existe a sensibilidade da região de interface entre a amostra e a máquina de ensaio, com o risco de ruptura da amostra na região da garra.

Em TERRIEN (1980) mostra-se uma curva tensão-deformação obtida de ensaios em corpos de prova cilíndricos (Figura 1.13). Duas fases distintas destacamse: antes da tensão de pico e após a tensão de pico.

Antes do pico de tensão $\left(\sigma<\mathrm{f}_{\mathrm{T}}\right)$, a curva resposta é inicialmente uma reta que passa a uma forma não-linear a partir de aproximadamente $50 \%$ de $\mathrm{f}_{\mathrm{T}}$ devido às primeiras evoluções da danificação na interface entre as fases agregado-argamassa.

Depois do nível de pico a tensão cai fortemente, ocorrendo essa instabilidade pela formação de fissuras localizadas na média-escala. Sucessivos ciclos de descarga permitem constatar uma grande redução da rigidez no final do ensaio ( $\mathrm{E} \cong \mathrm{E}_{0} / 10$ ). 


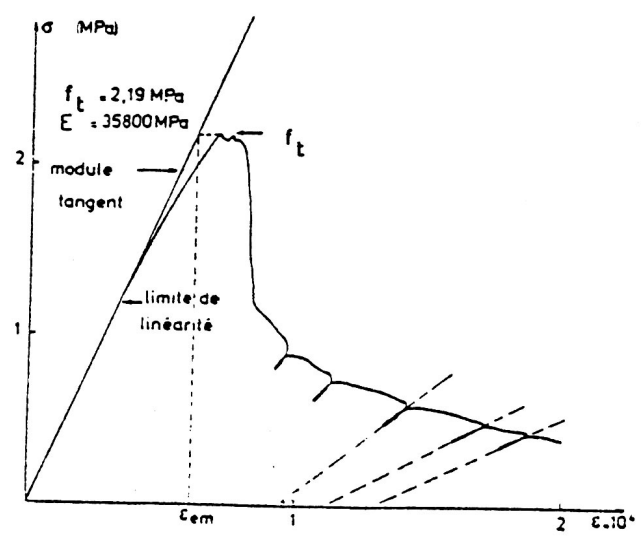

Figura 1.13 - Diagrama tensão-deformação em tração uniaxial [TERRIEN (1980)].

Outro trabalho importante no estudo do comportamento em tração do concreto foi efetuado por HEILMANN, HILSDORF \& FINSTERWALDER (1969). Os autores colocaram uma série de extensômetros ao longo do corpo de prova permitindo identificar deformações localizadas e dar uma melhor interpretação para a fase pós-pico de tensão. Da análise, detectou-se uma localização da deformação na zona onde acaba por se formar a fratura final. Nessa zona a deformação passa a evoluir um pouco mais rapidamente que nas outras regiões do corpo a partir de $60 \%$ de $\mathrm{f}_{\mathrm{T}}$, aproximadamente, acentuando-se a diferença próximo à tensão de pico. Além do pico, as deformações se concentram definitivamente na zona correspondente à fissura final e as zonas vizinhas sofrem descarregamento.

A razão principal para a localização é a existência de defeitos que levam a uma variação dos valores locais de resistência no interior do material.

Tendo-se em vista a resposta macroscópica observada e a localização em particular, HILLERBROG \& PETERSSON (1981) chegaram a uma conclusão sobre a representatividade do ensaio de tração:

- no início os defeitos se distribuem em todo o volume e nessas condições considera-se que o comportamento antes do pico é característico do material e pode ser representado num diagrama tensão-deformação (Figura 1.14a).

- devido à localização das deformações, a resposta depois do pico não é mais representativa do comportamento do material, mas sim da estrutura que constitui o 
corpo ensaiado. Assim, nessa fase o diagrama tensão-deformação não tem mais significado. Os autores aconselham que no regime pós-pico deve-se fazer referência à relação local $\sigma-\mathrm{w}$ (tensão - abertura da fissura) (Figura 1.14b).

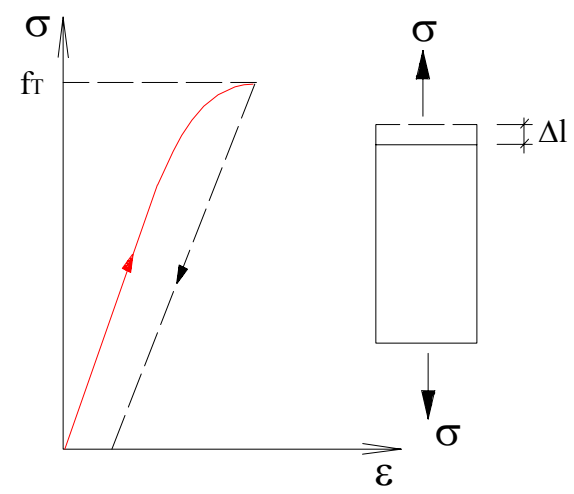

a)

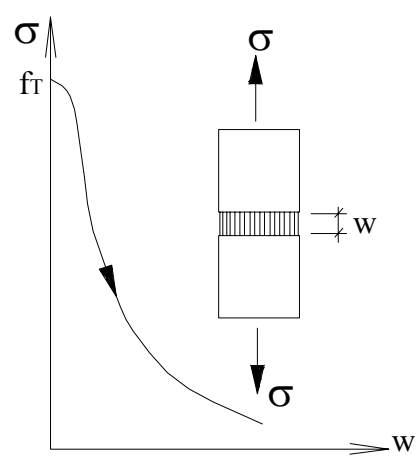

b)

Figura 1.14 - Análise do comportamento em tração segundo HILLERBORG \& PETERSSON (1981).

Com relação à influência da forma e do volume dos corpos de prova, cilíndricos ou prismáticos, sobre a resistência à tração direta, KADLECEK \& SPETLA (1967) e L'HERMITE (1973) realizaram alguns estudos.

No primeiro trabalho, os resultados apresentados indicam uma fraca influência da forma e uma influência marcante do volume. A tensão na ruptura é comparativamente menor quando o volume é maior e existe uma dispersão maior de resultados para pequenos volumes. Ambos os fenômenos, que constituem o chamado efeito escala, explicam-se pela quantidade de defeitos caracterizados na médiaescala.

No segundo trabalho, foram realizados ensaios de tração direta e de flexão em três e quatro pontos de prismas diferentes. Os resultados mostraram uma disparidade evidente entre as resistências obtidas para os dois tipos de ensaio (tração direta e flexão) ligados notadamente à combinação da distribuição de defeitos e gradiente de tensões, no caso do ensaio de flexão. Nos ensaios de flexão para uma amostra maior, o efeito do gradiente de tensões é fortemente diminuído, aproximando-se a 
resistência medida da resistência do ensaio de tração direta; porém o efeito de volume continua a se observar, diminuindo a resistência com o aumento do volume.

Como observações complementares, nota-se que o módulo de elasticidade longitudinal do concreto na tração é maior que na compressão, sendo que para o coeficiente de Poisson vale o inverso.

Vale ressaltar, pelo que foi observado, que o processo de danificação na tração uniaxial gera isotropia transversal no concreto pelos mesmos motivos comentados para o caso de compressão uniaxial.

\subsubsection{Comportamento Macroscópico do Concreto em Solicitações Multiaxiais}

De maneira clássica, o tensor de tensões pode ser decomposto da seguinte forma:

$\sigma=\mathrm{p} \mathbf{I}+\mathbf{S}$

onde pI é a parte hidrostática ou esférica do tensor de tensões e $\mathbf{S}$ é a sua parte desviadora.

Naturalmente, solicitações aplicadas ao concreto podem gerar estados de tensão de natureza hidrostática, desviadora ou uma combinação delas. MAZARS (1984) procura estabelecer uma correspondência entre a natureza do estado de tensão solicitante e o modo de danificação. No caso das solicitações multiaxiais, além dos modos descritos no item 1.3.2, acrescenta-se o modo de consolidação, associado na compressão à parte hidrostática do tensor de tensões conforme se descreve em seguida.

\subsubsection{Modos de danificação com vistas às solicitações multiaxiais}

Segundo MAZARS (1984), nas solicitações multiaxiais três modos de danificação na média-escala são considerados fundamentais:

- decoesão na interface matriz-agregado, ou na matriz somente, com modos de abertura dos tipos I e misto, I e II; 
- decoesão por escorregamento segundo o modo II (ou eventualmente III ou II + III)

- consolidação (ou diminuição da porosidade) por colapso da estrutura microporosa.

Tendo-se em vista os modos de danificação, Mazars identifica três categorias de solicitação, que estão descritas a seguir.

\subsubsection{Solicitações - Tipo A}

São solicitações que levam ao desenvolvimento de modos de abertura do tipo I, as quais normalmente ocorrem quando alongamentos no material não estão impedidas. As aberturas em modo I se orientam perpendicularmente às direções das extensões, como se observa facilmente nos ensaios uniaxiais de tração e de compressão. Nos casos de compressão biaxial, os planos de ruptura são paralelos ao plano de carregamento e perpendiculares à direção de extensão.

Qualquer processo que se superponha à compressão biaxial e que favoreça a propagação em modo I implica em diminuição da resistência do material.

Se em princípio a resistência à compressão uniaxial aumenta num estado de compressão em direção biaxial, quando se impõe uma tensão de tração na terceira direção, aquela resistência diminui quase que linearmente com o incremento da tração ; isso pode ser verificado na figura 1.15.

\begin{tabular}{|c|c|c|c|c|c|c|}
\hline$\sigma_{3} / \mathbf{f}_{\mathbf{T}}$ & 0 & 0,2 & 0,4 & 0,6 & 0,8 & 1 \\
\hline$\sigma_{1 \text { máx }} / \mathbf{f}_{\mathbf{C}}$ & 1,6 & 0,5 & 0,25 & 0,15 & 0,06 & 0 \\
\hline
\end{tabular}

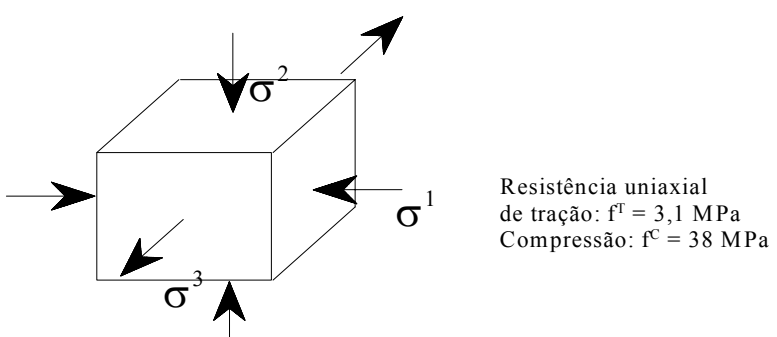

Figura 1.15 - Influência de uma tensão de tração $\left(\sigma_{3}\right)$ sobre o comportamento de um cubo comprimido nas outras duas direções $\left(\sigma_{1}=\sigma_{2}\right)$ [MAZARS (1984)]. 


\subsubsection{Solicitações - Tipo B}

Um estado desviador de tensão superposto a uma forte compressão hidrostática não possibilita, pela ausência de alongamentos, abertura de fissuras em modo I, mas essencialmente em modo II e talvez III. A evolução do dano se apresenta estável em boa parte do processo de carregamento pois as irregularidades das bordas das fissuras demandam maior consumo de energia para atingir a condição de escorregamento. O comportamento macroscópico é do tipo dúctil, num sentido comparável àquele obtido nos metais e para os quais a parte desviadora das tensões tem uma grande importância.

Ilustra-se na figura 1.16 um estado axissimétrico de tensões, evidenciando-se o aumento da resistência e deformabilidade na direção do carregamento (direção 3) com o aumento do confinamento nas direções perpendiculares a ele (direções 1 e 2). Neste caso observa-se o comportamento de isotropia transversal do material tendo as direções 1 e 2 as mesmas propriedades.

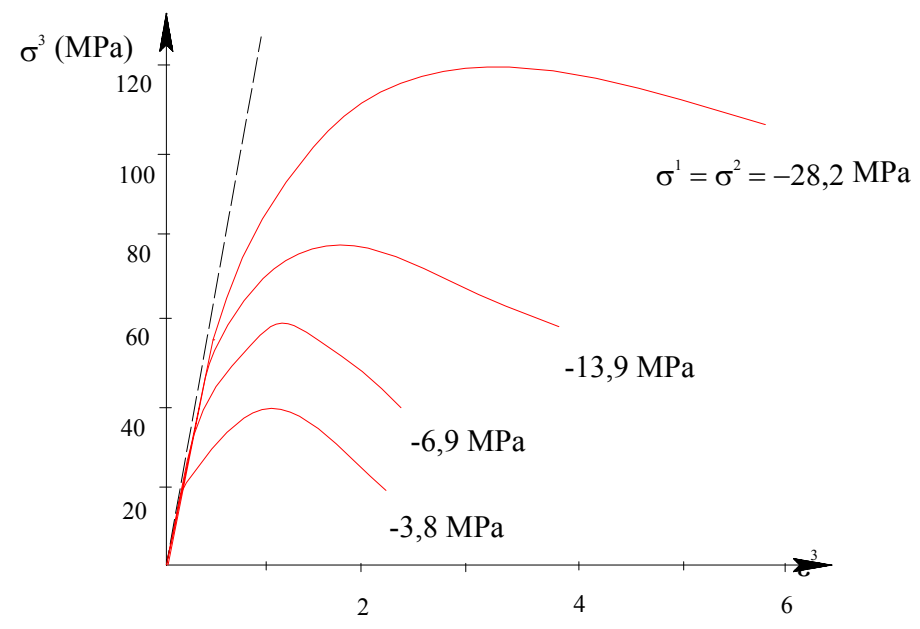

Figura 1.16 - Influência da tensão de confinamento em ensaios triaxiais [PROENÇA (1988)].

VAN MIER (1984) apresenta curvas tensão-deformação de alguns ensaios sob condições de deformação plana $\left(\varepsilon_{2}=0\right)$ (Figura 1.17). Todos os testes foram executados com carregamento perpendicular à direção de moldagem. Dos testes conclui-se que há um aumento da resistência com o aumento da tensão de confinamento $\left(\sigma_{3}=\beta \sigma_{1}, \beta>0\right)$. Também ocorre um acréscimo do nível de 
deformação relacionada à tensão de pico $\left(\sigma_{1} \times \varepsilon_{1}\right)$, e o ramo 'softening' apresenta uma forma similar para os três níveis de confinamento indicando um mesmo tipo de ruptura.

Cálculos do coeficiente de Poisson inicial de testes de deformação plana indicam um valor de $0,10-0,15$, que é menor que o valor em compressão uniaxial. Provavelmente a orientação de microfissuras decorrente da direção de moldagem é importante nesse contexto.

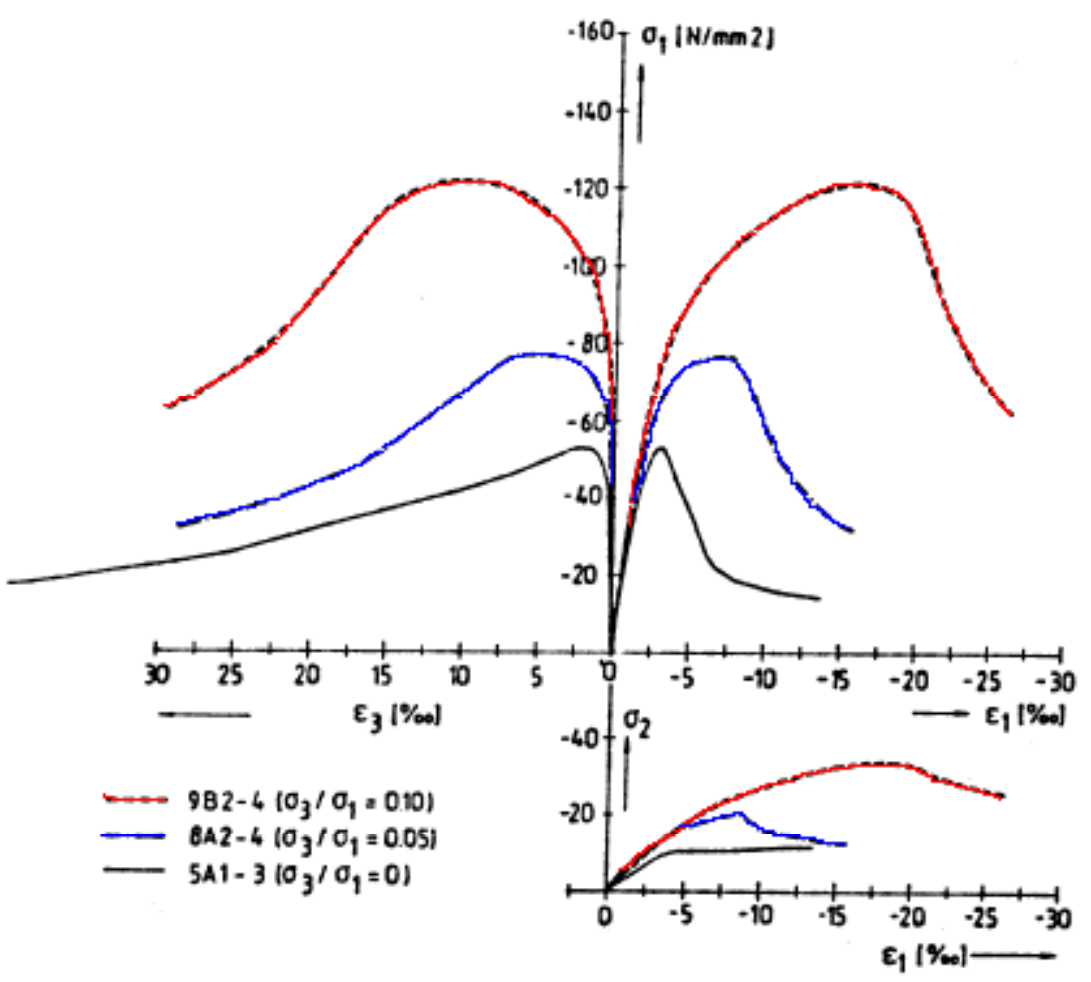

Figura 1.17 - Curvas tensão-deformação para testes de deformação plana com aumento de confinamento $\sigma_{3}=\beta \sigma_{1}(\beta=\mathbf{0} ; \mathbf{0 , 0 5} ; 0,10)$ [VAN MIER (1984)].

No caso de compressão triaxial, Van Mier apresenta curvas tensãodeformação (Figura 1.18) para três níveis da tensão $\sigma_{2}\left(\sigma_{2}=\alpha \sigma_{1} \operatorname{com} \alpha=0,05\right.$; $0,10 ; 0,33)$ e confinamento $\sigma_{3}=0$ e $\sigma_{3}=0,05 \sigma_{1}$, buscando ilustrar a influência da tensão principal intermediária.

A diferença no comportamento pós-pico é visível. Os resultados mostram que pode-se obter um acréscimo da resistência acompanhada de maior ou menor ductilidade dependendo da proporção entre as tensões. 


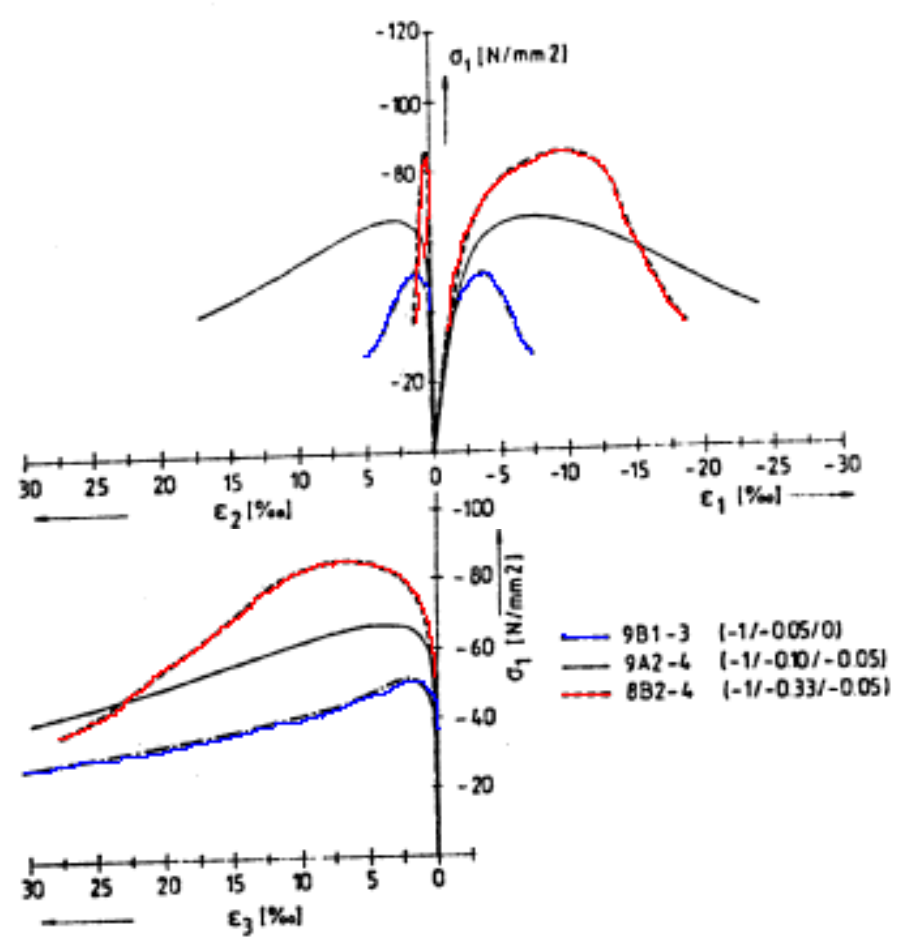

Figura 1.18 - Curvas tensão-deformação para testes com diferentes níveis de tensão intermediária. Testes com $\sigma_{3}=0$ e $\sigma_{3}=0,05 \sigma_{1}$ com carregamento paralelo à direção de moldagem [VAN MIER (1984)].

No concreto, a anisotropia inicial induzida pelo dano gerado durante a moldagem e processo de cura do espécime, se manifesta visivelmente na resposta tensão-deformação na medida em que a danificação evolui. Os efeitos são mais ou menos pronunciados dependendo da proporção entre as componentes de tensão no carregamento triaxial. Na figura 1.19, são mostradas as curvas tensão-deformação para espécimes com os seguintes carregamentos: $\sigma_{1} / \sigma_{2} / \sigma_{3}=-1 /-0,33 /-0,05$ e -1 / -0,33 / -0,10. Para cada proporção um par de espécimes foi carregado paralelamente e perpendicularmente à direção de moldagem. A resposta pré-pico dos diagramas é afetada pela orientação do campo de dano inicial com relação ao estado de tensão governante. 


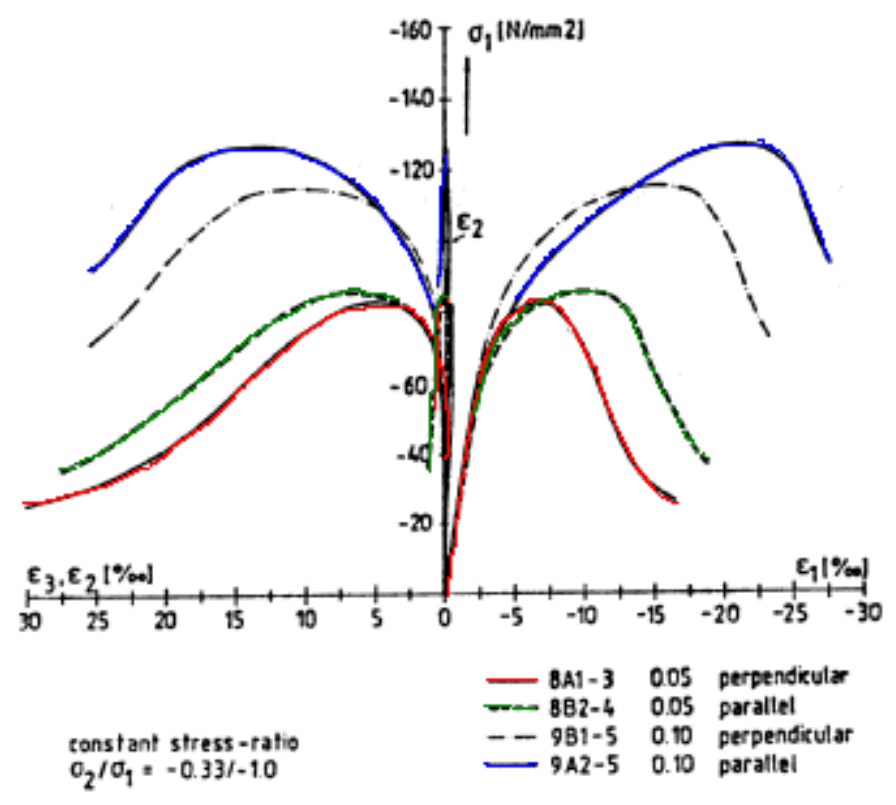

Figura 1.19 - Influência da anisotropia inicial no comportamento tensão-deformação triaxial [VAN MIER (1984)].

Quanto aos valores de resistência de pico, os resultados das séries triaxiais não mostram efeito significativo da orientação do carregamento em relação ao campo de dano inicial. Entretanto, para altos níveis de confinamento existe uma indicação que um pequeno acréscimo da resistência ocorre quando carregamento é aplicado paralelamente à direção de moldagem.

Mudando-se a direção do campo de dano inicial em relação ao carregamento triaxial aplicado, observa-se um incremento da ductilidade para carregamento paralelo à direção de moldagem. Nota-se em particular um significativo incremento da deformação nos níveis de tensão de pico.

\subsubsection{Solicitação - Tipo C}

É o caso de compressão hidrostática. Na figura a seguir apresenta-se a resposta carga hidrostática contra variação volumétrica obtida por BALMER (1949) apud MAZARS (1984).

\footnotetext{
${ }^{4}$ BALMER, G. G. . Shearing strenght of concrete under high triaxial stress - Computation of Mohr's Envelope as a curve. Structural Research Laboratory Report n. SP 23, Denver, 1949.
} 


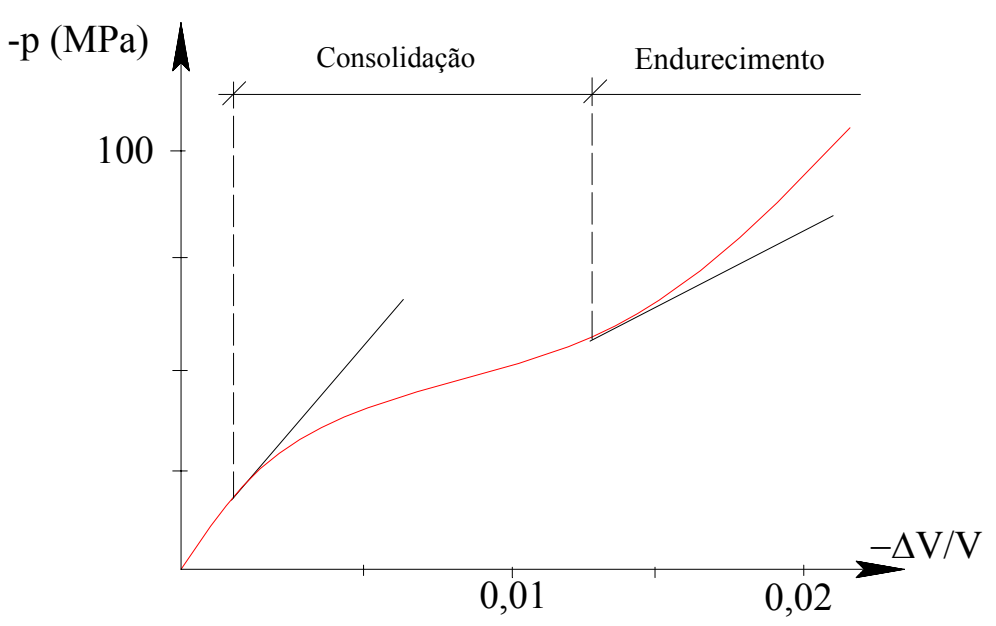

Figura 1.20 - Evolução da variação volumétrica sob carregamento hidrostático segundo BALMER (1949).

Três fases distintas aparecem:

- uma fase elástica sem degradações;

- uma fase de consolidação em correspondência à ruína da estrutura microporosa da pasta de cimento hidratado;

- uma fase de endurecimento ligada ao comportamento do material consolidado.

Para o concreto sujeito à compressão hidrostática parece não existir, teoricamente, limites para a evolução das tensões. O caminho de tensão deve, naturalmente, atingir um limite de ruptura, mas provavelmente esse limite acontecerá dentro de uma região de compressão triaxial intensa.

Por outro lado, o caso de tração hidrostática não parece ter sido estudado, pois esse tipo de solicitação é muito difícil de ser realizado experimentalmente. Tendo-se em vista o comportamento em tração uniaxial, é razoável imaginar que o comportamento sob tração hidrostática seja do tipo elástico-frágil.

Dos ensaios observados, conclui-se que não parece haver um limite ou critério inicial de dano, baseado em tensão ou deformação, abaixo do qual o material permanece íntegro. Porém, parece razoável admitir, para efeitos de formulação teórica, um certo limite onde a danificação passa a ter efeitos significativos no comportamento do material. 
Além disso, estes mesmos ensaios nos conduzem a imaginar que é algo razoável simular o comportamento do concreto com as hipóteses de material inicialmente isótropo e com anisotropia induzida pelo dano, em particular a isotropia transversal. É claro que pelo que se observou, a rigor, nos casos mais gerais, a ortotropia induzida pelo dano seria mais plausível, contudo essa hipótese levaria à formulação de um modelo matemático mais complexo, que dependendo do caso não seria interessante em termos de identificação paramétrica, emprego prático e custo teórico da formulação.

Para finalizar, resume-se na tabela a seguir a classificação das solicitações em correspondência aos modos de dano, segundo Mazars:

Tabela 1.1 - Classificação dos comportamentos relacionando o modo de danificação e o tipo de solicitação [MAZARS (1984)].

\begin{tabular}{|c|c|c|c|}
\hline $\begin{array}{c}\text { Tipo de } \\
\text { Solicitação }\end{array}$ & Modo de Dano Local & Particularidades & Exemplo de Comportamento \\
\hline $\begin{array}{l}\text { A: Uniaxial, } \\
\text { biaxial, triaxial } \\
\text { com tração ou } \\
\text { fraca compressão } \\
\text { em ao menos uma } \\
\text { direção }\end{array}$ & $\begin{array}{l}\text { Microfissuração } \\
\text { principalmente } \\
\text { ao redor dos grãos } \\
\text { Modo I ou I + II }\end{array}$ & $\begin{array}{c}\text { - existência de uma } \\
\text { extensão em ao } \\
\text { menos uma direção } \\
\text { principal } \\
\text { - comportamento } \\
\text { mais ou menos } \\
\text { "frágil" }\end{array}$ & ${ }^{1}-\sigma^{1(\mathrm{MPa})}$ \\
\hline $\begin{array}{l}\text { B: Triaxial de } \\
\text { Compressão - } \\
\text { superpondo-se } \\
\text { uma pressão } \\
\text { hidrostática } \\
\text { elevada à um } \\
\text { estado desviador }\end{array}$ & $\begin{array}{l}\text { Microfissuração } \\
\text { principalmente } \\
\text { em modo II }\end{array}$ & $\begin{array}{c}\text { - nenhuma } \\
\text { extensão } \\
\text { - comportamento } \\
\text { "dúctil" }\end{array}$ & 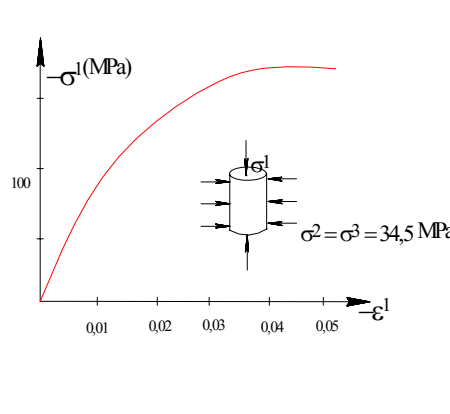 \\
\hline $\begin{array}{l}\text { C: Triaxial- } \\
\text { Essencialmente } \\
\text { pressão } \\
\text { hidrostática }\end{array}$ & $\begin{array}{l}\text { Consolidação da } \\
\text { estrutura microporosa }\end{array}$ & $\begin{array}{l}\text { - comportamento } \\
\text { com várias fases } \\
\text { ( amolecimento } \\
\text { seguido de } \\
\text { endurecimento) }\end{array}$ & ${ }_{0.02}-\overline{\Delta V} / \mathrm{V}$ \\
\hline
\end{tabular}




\subsubsection{Modos de ruptura}

No item 1.3.2 comentaram-se os mecanismos de danificação do concreto em estados uniaxiais. Neste item tratam-se dos modos de ruptura em estados multiaxiais, sendo que, na maior parte dos casos, em decorrência da localização de deformações, os modos de ruptura derivam de mecanismos de danificação similares àqueles dos estados uniaxiais.

\section{- Estados de Compressão Triaxial}

Os comportamentos pós-pico distintos das curvas tensão-deformação (Figura 1.18) para diferentes estados triaxiais indicam, de acordo com um ramo de amolecimento mais ou menos pronunciado, processos de fraturamento localizado ou distribuído respectivamente. Por exemplo, enquanto no espécime com $\sigma_{1} / \sigma_{2} / \sigma_{3}=$ 1 / -0,33 / -0,05 evidencia-se uma banda de cisalhamento localizada, num outro espécime, com $\sigma_{1} / \sigma_{2} / \sigma_{3}=-1 /-0,10 /-0,05$, apresenta-se um panorama de fissuração distribuída. Uma observação mais detalhada revela que esse panorama de fissuração é o resultado de cruzamentos entre diversas bandas de cisalhamento.

- Estados Biaxiais Compressão-Compressão ou Compressão-Tração

Nos casos de compressão-tração, os experimentos mostram ruptura por fraturamento perpendicular à direção da tensão de tração.

De um modo geral, a ruptura do concreto em estados biaxiais ocorre por separação ortogonal à direção de tração máxima ou de deformação (alongamento) máxima (Figura 1.21). Observa-se que as deformações por alongamento também são importantes no estabelecimento de critérios de ruptura para o concreto e que nesses casos o dano gerado implica num comportamento de isotropia transversal marcante. 


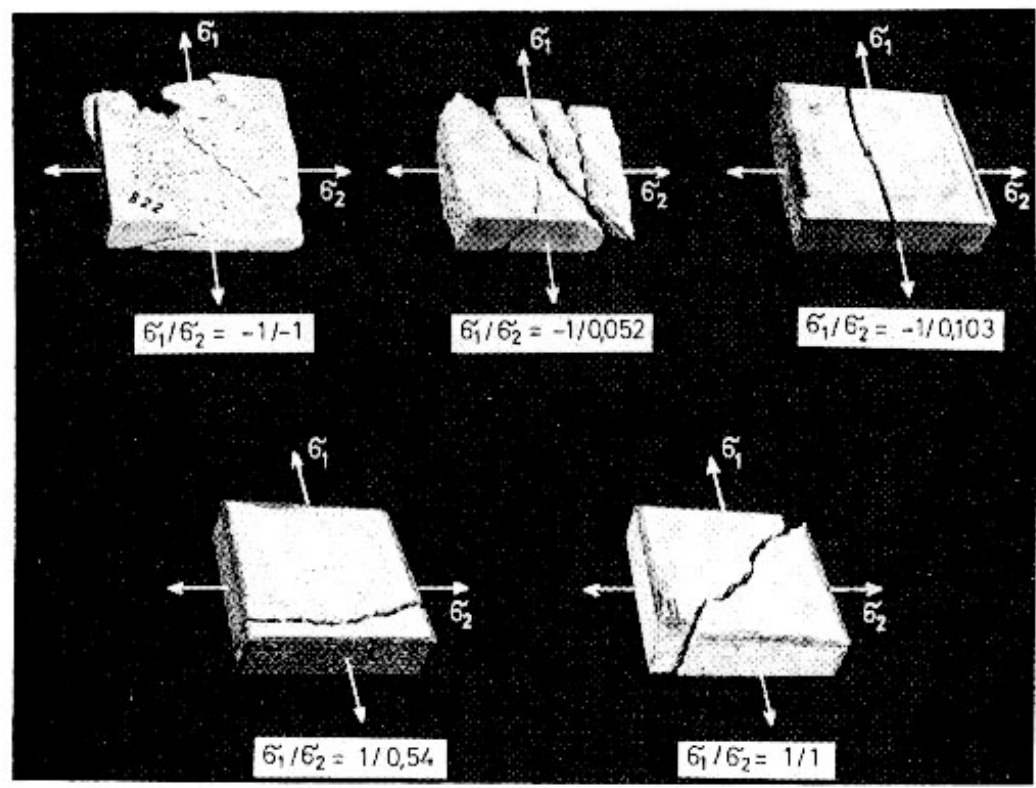

Figura 1.21 - Modos de ruptura de espécimes de concreto sujeitos a estados biaxiais de tensão [KUPFER, HILSDORF \& RUSCH (1969)].

\subsubsection{Mudanças de volume}

Como já foi comentado anteriormente, espécimes carregados em compressão triaxial podem romper ao longo de um plano de cisalhamento bem definido ou pela formação de uma distribuição de pequenos planos de cisalhamento inclinados em várias direções. Em função da estrutura granular do material, escorregamentos nos planos de cisalhamento implicam numa mudança de volume. Assim sendo, nas solicitações triaxiais de compressão a ocorrência de uma grande variação de volume além do nível de tensão de pico $\left(\sigma_{1}\right)$ não é surpresa tendo-se em vista o modo de ruptura.

As variações volumétricas associadas aos processos de escorregamento nos materiais granulares, como o concreto, podem ser explicadas por modelos simples ilustrados nas figuras a seguir. 


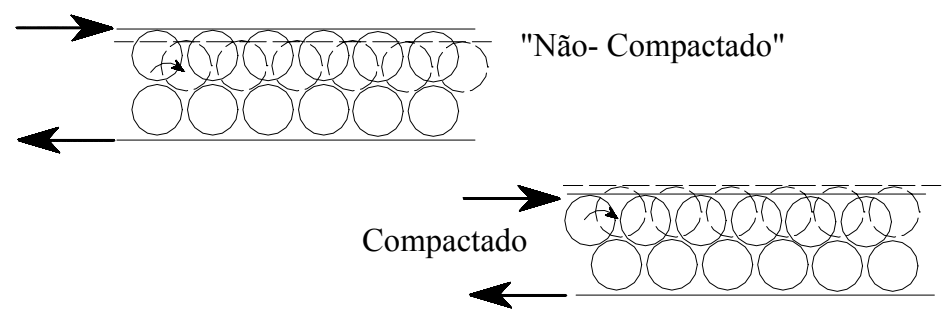

Figura 1.22 - Modelos de variação volumétrica para materiais granulares.

Para avaliar a mudança no volume de espécimes em concreto, VAN MIER (1984) realizou experimentos triaxiais com razão de confinamento constante. Três espécimes foram carregados com $\sigma_{2}$ de compressão crescente, mantendo-se $\sigma_{3}=0,05$ $\sigma_{1}$ e analogamente outros três espécimes com $\sigma_{3}=0,10 \sigma_{1}$. Os seis espécimes foram todos submetidos a carregamentos paralelos com relação à direção de moldagem.

Foram analisadas curvas de deformação volumétrica $\varepsilon_{\mathrm{V}}$ contra a deformação de compressão maior $\varepsilon_{1}$. Inicialmente verificou-se uma compactação, sendo a relação entre $\varepsilon_{\mathrm{V}}$ e $\varepsilon_{1}$ não-linear. Com o acréscimo de $\varepsilon_{1}$, ocorreu um decréscimo da taxa de compactação e um volume mínimo foi observado mesmo antes que o nível de tensão de pico seja atingido. A posterior taxa de crescimento de volume é maior para espécimes com nível de confinamento menor.

Em estados biaxiais de compressão, ocorre um acréscimo de volume com o aumento das tensões de compressão nas proximidades do ponto de resistência limite.

Com relação à ductilidade, a variação dessa característica depende das combinações de solicitações. Em estados triaxiais de compressão a ductilidade é maior, porém se ao menos uma das tensões for de tração a ductilidade é comparativamente menor.

\subsubsection{Influência das Condições de Ensaio}

Por condições de ensaio, além dos problemas relacionados ao próprio material (composição), entende-se geometria dos corpos ensaiados e a natureza dos contatos entre a máquina e o corpo. 
Em MAZARS (1984) mostra-se um estudo comparativo do efeito de diferentes tipos de contatos corpo-máquina, realizado por GLOMB \& PATAS (1972). A partir de ensaios em cubos de $20 \times 20$ × $20 \mathrm{~cm}$, investigou-se a possível dependência da resistência à compressão simples $\left(\mathrm{f}_{\mathrm{C}}\right)$ da natureza da interface entre o corpo e o prato da máquina. Da análise constata-se de fato uma dependência, verificando-se ainda que o valor da resistência pode diminuir por volta de $40 \%$ pela utilização de duas camadas de teflon parafinado na interface prato-corpo de prova.

A dependência verificada se dá porque o atrito na interface restringe a livre deformação lateral do corpo de prova, criando-se assim um estado triaxial naquela região. Esse fenômeno é, aliás, responsável pela ruptura em forma de cone observada nas vizinhanças daquela região.

Essa forma de ruptura é a mais localizada pois quanto mais alto é o corpo de prova menores são as repercussões das condições de contato na zona central.

\subsubsection{Influência da Trajetória do Carregamento}

Solicitações de natureza cíclica colocam em evidência o caráter unilateral da resposta do concreto, TERRIEN (1980). No ensaio realizado por aquele autor, um corpo de prova em concreto foi inicialmente tracionado e em seguida submetido à compressão (Fig.1.23a). Constatou-se o fechamento das fissuras abertas pela tração e a recuperação de rigidez no regime de compressão. Outro trabalho que procurou analisar esse efeito foi elaborado por MAZARS, BERTHAUD \& RAMTANI (1990), sendo estudados casos de solicitação uniaxial em blocos e de flexão em vigas, alcançando as mesmas conclusões obtidas por Terrien.
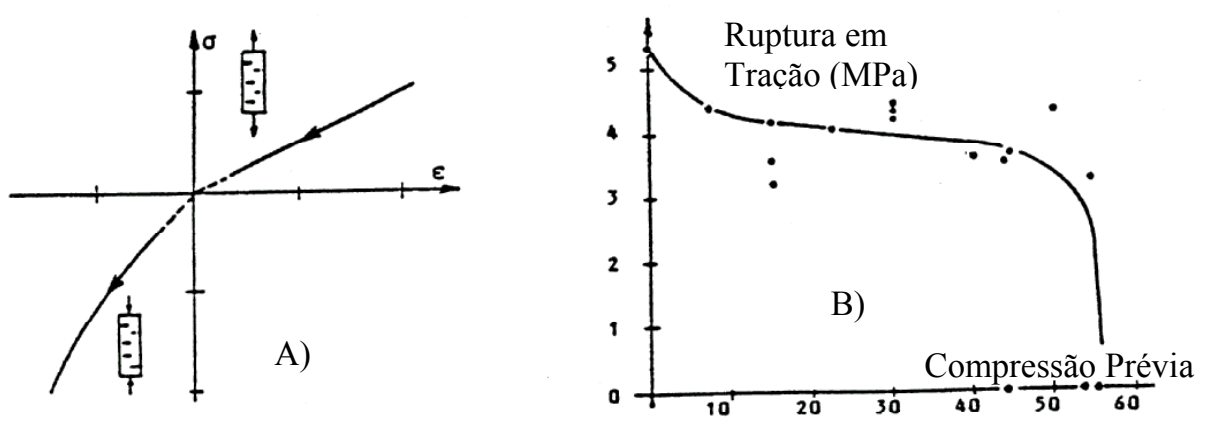

Figura 1.23 - Dois diagramas ilustrando a influência da trajetória do carregamento. 
Já em TOMACHEVSKY et al.(1975) ${ }^{5}$ apud MAZARS (1984), mostra-se que uma compressão prévia enfraquece a resistência à tração numa direção a ela perpendicular (Figura 1.23b).

Em casos de carregamento proporcional o modo de danificação permanece o mesmo, porém com a aplicação de carregamento não-proporcional o modo inicial pode ser modificado com a mudança na ordem de aplicação da solicitação. Esse fato pode ser deduzido dos resultados da Fig. 1.24, onde cubos com pré-carregamento hidrostático de compressão $\left(\sigma_{1}=\sigma_{2}=\sigma_{3}\right)$ passam a ser solicitados por um estado de tensões com evolução de $\sigma_{1}$ e manutenção de $\sigma_{2}$ e $\sigma_{3}$ em 29,30 MPa.

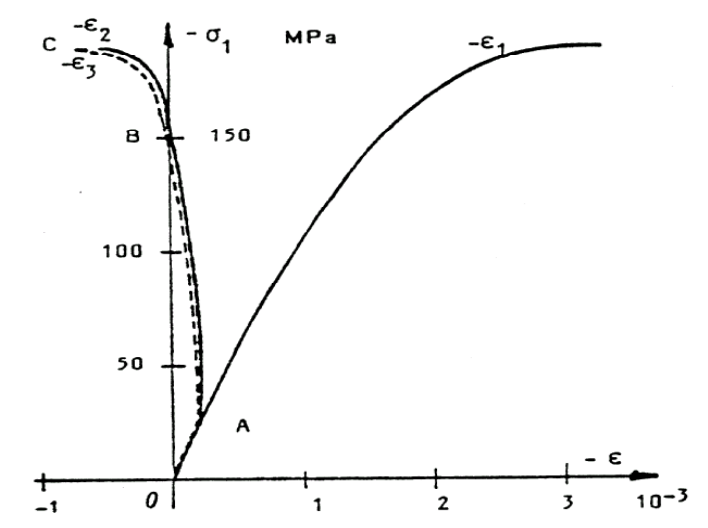

Figura 1.24 - Comportamento sob carregamento de compressão com restrição lateral $\left(\sigma_{2}=\sigma_{3}=29,30 \mathrm{MPa}\right)$ [BERTACCHI \& BELOTTI (1972)].

Três fases distintas surgem:

- fase OA (carregamento hidrostático): elasticidade e consolidação;

- fase AB: extensões impedidas ( ruptura em modo II ou III);

- fase BC: extensões possíveis (rupturas em modo I ou misto com instabilidade e ruptura).

Nos ensaios usuais, aplica-se de início o confinamento transversal e em seguida inicia-se o carregamento axial. Nesses casos, a fase OA não é observada.

${ }^{5}$ TOMACHEVSKY, E. G., DROUET, A. \& DESPERLES, P. J. Recherche de la diminution de résistance en traction du béton par détection d'émission d'ondes de contrainte. Rapport Interne C. E. A., 1975. 


\subsubsection{Comportamento do Concreto Fissurado em Relação à Resistência ao Cisalhamento}

Comenta-se brevemente neste item o comportamento do concreto fissurado quando submetido a esforços de cisalhamento.

FIGUEIRAS (1983) já chama a atenção sobre investigações experimentais que mostraram a importante contribuição do concreto fissurado na resistência ao cisalhamento, no caso de elementos de concreto armado. Dois mecanismos estão presentes nesse comportamento, são eles: a chamada ação de "pino" e a transferência de tensão de cisalhamento entre as faces das bordas das fissuras.

Enquanto que o primeiro é particular para o concreto armado, o segundo é de maior interesse no contexto deste trabalho.

As superfícies das fissuras são usualmente rugosas e irregulares, geralmente contornando os agregados graúdos do concreto. As superfícies rugosas das faces das fissuras podem transferir tensões de cisalhamento através da fricção e do engrenamento dos agregados, como se ilustra na figura 1.25. A aplicação de esforço cisalhante $\mathrm{v}$ causa um deslocamento $\mathrm{d} \mathrm{e}$, devido à irregularidade da superfície tende $\mathrm{a}$ provocar uma separação das superfícies da fissura ou, dilatação w do meio. Resultados experimentais mostraram que a abertura inicial de fissura $\left(\mathrm{w}_{0}\right)$ é a variável principal que afeta a ação de engrenamento dos agregados. Grandes aberturas iniciais de fissuras implicam em menor rigidez e resistência ao cisalhamento.

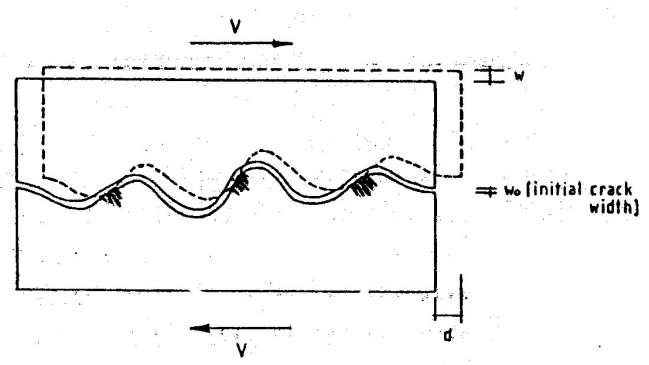

Figura 1.25 - Mecanismo de engrenamento dos agregados para a transferência de tensões de cisalhamento através da fissura [FIGUEIRAS (1983)].

Algumas aproximações para se levar em conta a perda de rigidez ao cisalhamento podem ser encontradas na literatura. Entre elas, pode-se citar a proposta 
de HAND, PECKNOLD \& SCHNOBRICH (1973) que modelaram a rigidez ao cisalhamento do concreto (módulo de elasticidade transversal) como um valor constante reduzido em relação ao valor inicial. Entretanto, para FIGUEIRAS (1983), o valor do módulo de elasticidade transversal decrescendo linearmente com a deformação normal ao plano fissurado (representando a abertura da fissura) constitui-se numa melhor aproximação no confronto com resultados experimentais.

A figura 1.26 ilustra uma comparação de outras expressões propostas para a variação do módulo de elasticidade transversal do concreto fissurado.

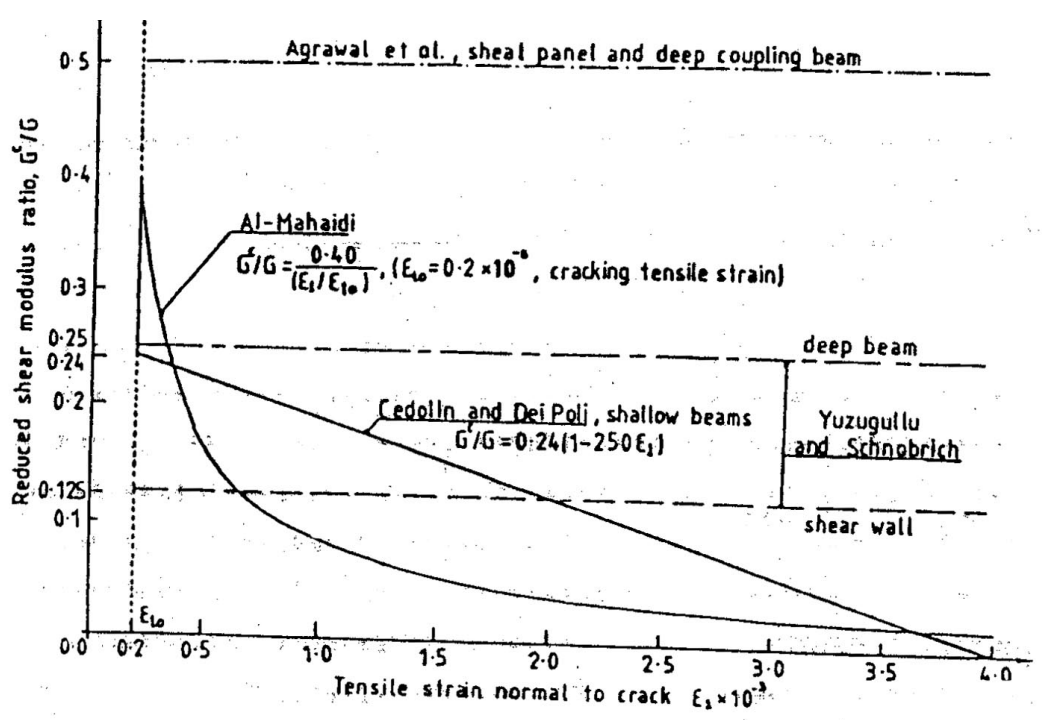

Figura 1.26 - Comparação de vários diagramas propostos para o módulo de elasticidade transversal do concreto fissurado. [ASCE(1981) ${ }^{6}$ apud FIGUEIRAS (1983)].

\footnotetext{
${ }^{6}$ ASCE. Committee on concrete and masonry structures, Task Committee on Finite Element Analysis of Reinforced Concrete Structures: A State-of-the-Art Report on Finite Element Analysis of Reinforced Concrete Structures, ASCE Spec. Pub., 1981.
} 


\section{MEIOS ELÁSTICOS DANIFICADOS ANISÓTROPOS COM RESPOSTA BIMODULAR}

\subsection{INTRODUÇÃO}

Neste capítulo, inicialmente faz-se uma breve revisão da formulação de modelos constitutivos para meios elásticos anisótropos com resposta bimodular proposta por CURNIER, HE \& ZYSSET (1995). Logo após, propõe-se uma extensão daquela formulação para levar em conta a danificação progressiva do meio. Como exemplo das possibilidades que se abrem a partir da formulação para meios danificados, aborda-se o caso particular de um meio elástico danificado com isotropia transversal e bimodularidade induzidas pelo dano, o qual será utilizado na formulação do modelo para o concreto proposto neste trabalho.

$\mathrm{Na}$ seqüência do capítulo, apresentam-se alguns modelos de dano para o concreto propostos na literatura, que se caracterizam como modelos isótropos ou anisótropos, destacando-se a diferença conceitual entre eles. Vale ressaltar que para cada um dos modelos ilustrados, a apresentação limita-se aos tensores constitutivos, que levam em conta a danificação do material, não sendo objeto de análise toda a sua formulação matemática. 


\subsection{ELASTICIDADE LINEAR DE MEIOS COM ANISOTROPIA E RESPOSTA BIMODULAR}

Muitos materiais compósitos, reforçados com fibras, apresentam-se claramente como meios anisótropos e bimodulares [JONES (1977)]. Já compósitos quase-frágeis, como o concreto, pertencem a uma categoria de materiais que podem ser considerados inicialmente isótropos e unimodulares, porém, quando danificados passam a exibir anisotropia e diferentes rigidezes em tração e em compressão [MAZARS, BERTHAUD \&RAMTANI (1990)]. Nesta seção a questão do dano ainda não é considerada, sendo o destaque dado aos aspectos de anisotropia e resposta bimodular. Nesse sentido, apresenta-se, de forma resumida, a formulação de leis constitutivas, proposta por CURNIER, HE \& ZYSSET (1995) para materiais com isotropia ou anisotropia elástica, que possuem diferentes comportamentos em tração e em compressão. A formulação é limitada ao regime de pequenas deformações.

Em hiperelasticidade, o potencial quadrático, envolvendo um tensor de elasticidade constante, e a lei linear entre tensão e deformação, dele derivada, apresentam as seguintes características:

$$
\begin{array}{ll}
\mathrm{W}(\varepsilon)=\frac{1}{2} \mathbf{E}_{0} \varepsilon \cdot \varepsilon & \mathrm{W} \in \ell^{2}(\varepsilon, \mathfrak{R}) ; \\
\sigma(\varepsilon)=\mathbf{E}_{0} \varepsilon & \sigma \in \ell^{1}(\varepsilon, \wp) \\
\mathbf{E}_{\mathbf{0}}=\text { constante } & \mathbf{E}_{\boldsymbol{0}} \in \ell^{0}\left(\varepsilon, \boldsymbol{E}_{\boldsymbol{0}}\right)
\end{array}
$$

Como indicado na (2.1) W é um campo de argumento tensorial de valor escalar real duas vezes continuamente diferenciável; na (2.2) $\sigma$ é um campo tensorial de segunda ordem uma vez diferenciável e na (2.3) $\mathbf{E}_{\mathbf{0}}$ é campo tensorial de quarta ordem. No caso geral de anisotropia, o tensor constitutivo $\mathbf{E}_{\mathbf{0}}$ é simétrico e envolve 21 constantes do material.

Segundo a forma dual, o potencial de energia complementar, a relação constitutiva e o tensor de flexibilidade são dados por: 


$$
\begin{aligned}
& \mathrm{W}^{*}(\sigma)=\frac{1}{2} \mathbf{C}_{0} \sigma \cdot \sigma \\
& \varepsilon(\sigma)=\mathbf{C}_{0} \sigma \\
& \mathbf{C}_{\mathbf{0}}=\mathbf{E}_{0}^{-1}=\text { constante }
\end{aligned}
$$

As relações anteriores, para o caso de elasticidade linear com anisotropia, envolvem constantes ditas "volumétricas" $\lambda_{\mathrm{ab}}$ e de "cisalhamento" $\mu_{\mathrm{a}}$, que se reduzem àquelas de Lamè no caso de isotropia. As formas particulares que incluem isotropia, isotropia transversal e ortotropia, são as seguintes:

$$
\begin{aligned}
& \mathbf{W}(\varepsilon)=\frac{\lambda_{\mathrm{ab}}}{2} \operatorname{tr}\left(\mathbf{A}_{\mathrm{a}} \varepsilon\right) \operatorname{tr}\left(\mathbf{A}_{\mathrm{b}} \varepsilon\right)+\mu_{\mathrm{a}} \operatorname{tr}\left(\mathbf{A}_{\mathrm{a}} \varepsilon^{2}\right) \quad(\mathrm{a}, \mathrm{b}=1, \mathrm{~d}) \\
& \sigma(\varepsilon)=\lambda_{\mathrm{ab}} \operatorname{tr}\left(\mathbf{A}_{\mathrm{a}} \varepsilon\right) \mathbf{A}_{\mathrm{b}}+\mu_{\mathrm{a}}\left(\mathbf{A}_{\mathrm{a}} \varepsilon+\varepsilon \mathbf{A}_{\mathrm{a}}^{\mathbf{T}}\right) \\
& \mathbf{E}_{0}=\lambda_{\mathrm{ab}}\left[\mathbf{A}_{\mathrm{a}} \otimes \mathbf{A}_{\mathrm{b}}\right]+\mu_{\mathrm{a}}\left[\mathbf{A}_{\mathrm{a}} \otimes \mathbf{I}+\mathbf{I} \otimes \mathbf{A}_{\mathrm{a}}\right] \quad \lambda_{\mathrm{ab}}=\lambda_{\mathrm{ba}}
\end{aligned}
$$

onde $\mathrm{d}=1$ para isotropia (envolvendo as duas constantes de Lamè), $\mathrm{d}=2$ para isotropia transversal ( 5 constantes) e $\mathrm{d}=3$ para ortotropia ( 9 constantes). Os tensores $\mathbf{A}_{\mathbf{a}}$ e $\mathbf{A}_{\mathbf{b}}$ são: $\left(\mathbf{A}_{\mathbf{1}}=\mathbf{I}\right)$ para materiais isótropos, $\left(\mathbf{A}_{\mathbf{1}}=\mathbf{I}, \mathbf{A}_{\mathbf{2}}=\mathbf{A}\right)$ para materiais com isotropia transversal e $\left(\mathbf{A}_{1}=\mathbf{I}, \mathbf{A}_{\mathbf{2}}=\mathbf{A}, \mathbf{A}_{\mathbf{3}}=\mathbf{B}\right)$ para materiais ortótropos.

Tomando-se o caso de isotropia transversal como exemplo, o tensor I é o tensor identidade de segunda ordem e o tensor $\mathbf{A}$ é formado pelo produto tensorial do vetor perpendicular ao plano de isotropia transversal por ele mesmo. No caso de ortotropia, existem três vetores linearmente independentes que definem os planos de ortotropia, sendo os tensores A e B definidos por dois daqueles vetores e realizandose os produtos tensoriais deles por eles mesmos; há também um tensor associado à terceira direção, formado pelos tensores $\mathbf{I}, \mathbf{A}$ e $\mathbf{B}(\mathbf{C}=\mathbf{I}-\mathbf{A}-\mathbf{B})$.

As relações duais podem ser escritas na forma:

$$
\begin{aligned}
& \mathrm{W}^{*}(\sigma)=-\frac{v_{\mathrm{ab}}}{2 \mathrm{E}_{\mathrm{a}}} \operatorname{tr}\left(\mathbf{A}_{\mathrm{a}} \sigma\right) \operatorname{tr}\left(\mathbf{A}_{\mathrm{b}} \sigma\right)+\frac{1+\mathrm{v}_{\mathrm{aa}}}{2 \mathrm{E}_{\mathrm{a}}} \operatorname{tr}\left(\mathbf{A}_{\mathrm{a}} \sigma^{2}\right) \quad(\mathrm{a}, \mathrm{b}=1, \mathrm{~d}) \\
& \varepsilon(\sigma)=-\frac{v_{\mathrm{ab}}}{\mathrm{E}_{\mathrm{a}}} \operatorname{tr}\left(\mathbf{A}_{\mathrm{a}} \sigma\right) \mathbf{A}_{\mathrm{b}}+\frac{1+v_{\mathrm{aa}}}{2 \mathrm{E}_{\mathrm{a}}}\left(\mathbf{A}_{\mathrm{a}} \sigma+\sigma \mathbf{A}_{\mathrm{a}}^{\mathbf{T}}\right)
\end{aligned}
$$




$$
\mathbf{C}_{\mathbf{0}}=-\frac{v_{\mathrm{ab}}}{\mathrm{E}_{\mathrm{a}}}\left[\mathbf{A}_{\mathrm{a}} \otimes \mathbf{A}_{\mathrm{b}}\right]+\frac{1+v_{\mathrm{aa}}}{2 \mathrm{E}_{\mathrm{a}}}\left[\mathbf{A}_{\mathrm{a}} \otimes-\overline{\mathbf{I}}+\mathbf{I} \otimes \mathbf{A}_{\mathrm{a}}\right] \quad \frac{v_{\mathrm{ab}}}{\mathrm{E}_{\mathrm{a}}}=\frac{v_{\mathrm{ba}}}{\mathrm{E}_{\mathrm{b}}}
$$

As operações de produtos tensoriais entre tensores de segunda ordem inseridas aqui estão descritas no apêndice deste trabalho.

Por outro lado, considerando-se um caso unidimensional, a palavra “bimodular" refere-se a materiais cujas leis tensão-deformação unidimensionais são caracterizadas por diferentes módulos de elasticidade nos domínios de tração e de compressão.

A extensão de tais características para os casos bi e tridimensional é proposta no trabalho de CURNIER, HE \& ZYSSET (1995) e passa a ser descrita no que segue.

Para a modelagem de um material hiperelástico bimodular, considera-se que a densidade de energia potencial elástica $\mathrm{W}$ deva ser uma vez diferenciável continuamente (em qualquer ponto) porém diferenciável duas vezes continuamente apenas por partes. A relação tensão-deformação derivada de tal potencial tem continuidade (em qualquer ponto) e apresenta um tensor de elasticidade descontínuo em relação a uma hipersuperfície que contém a origem e que divide o espaço das deformações em regiões de tração e de compressão; nessas condições torna-se possível reproduzir uma resposta bimodular.

Seja, então, uma hipersuperfície definida no espaço das deformações por uma função de valor escalar, $g \in \ell^{1}(\varepsilon, \mathfrak{R}): g(\varepsilon)=0$ (Figura 2.1). Definem-se, então, os seguintes conjuntos:

$$
\begin{aligned}
& \not \subset:=\{\varepsilon \in \varepsilon \mid g(\varepsilon)=0\} \quad g \in \ell^{1}(\varepsilon, \mathfrak{R}) \\
& \varepsilon_{-}:=\{\varepsilon \in \varepsilon \mid g(\varepsilon)<0\}, \quad \bar{\varepsilon}_{-}:=\{\varepsilon \in \varepsilon \mid g(\varepsilon) \leq 0\} \\
& \varepsilon_{+}:=\{\varepsilon \in \varepsilon \mid g(\varepsilon)>0\}, \quad \bar{\varepsilon}_{+}:=\{\varepsilon \in \varepsilon \mid g(\varepsilon) \geq 0\} \\
& \varepsilon=\varepsilon_{-} \cup \varepsilon_{+} \cup \not ̧, \quad \varepsilon_{-} \cap \varepsilon_{+}=\varnothing, \quad \bar{\varepsilon}_{-} \cap \bar{\varepsilon}_{+}=\not \varnothing
\end{aligned}
$$




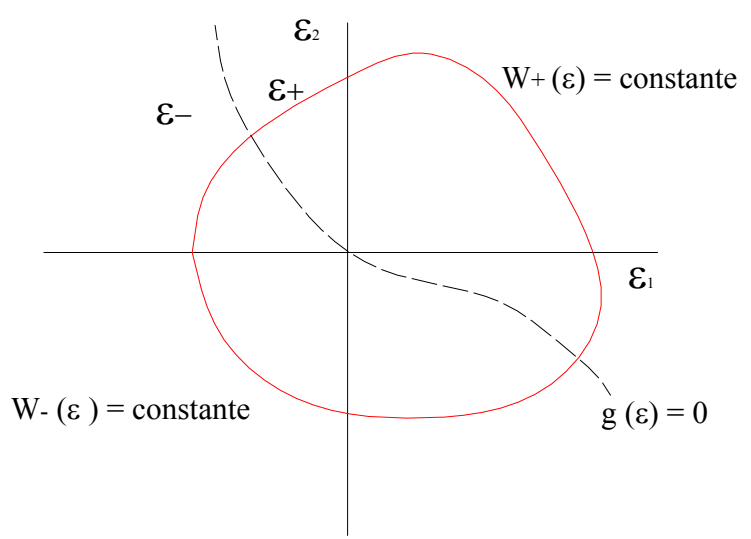

Figura 2.1 - Representação esquemática da subdivisão do espaço de deformações em subdomínios de compressão e de tração.

Por definição a hipersuperfície $\mathrm{g}(\varepsilon)=0$ deve conter a origem do espaço das deformações de modo que o tensor $\varepsilon=0$ pertence ao conjunto $\not{Z}$ :

$\mathbf{0} \in \not{\not} \Leftrightarrow g(\mathbf{0})=0$

A hipersuperfície serve, então, como critério para que o espaço das deformações resulte separado em partes, às quais corresponderão regimes predominantes de tração e regimes predominantes de compressão.

Entre as possibilidades de representação da hipersuperfície, um hiperplano, caracterizado por sua normal unitária (tensor de segunda ordem) $\mathbf{N}(\|\mathbf{N}\|=1)$, proporciona a representação mais simples no espaço das deformações. Nesse caso valem as relações:

$$
\begin{aligned}
& \not \subset:=\{\varepsilon \in \varepsilon \mid g(\varepsilon)=\mathbf{N} . \varepsilon=0\} \\
& \varepsilon_{-}:=\{\varepsilon \in \varepsilon \mid \mathbf{N} . \varepsilon<0\}, \quad \varepsilon_{+}:=\{\varepsilon \in \varepsilon \mid \mathbf{N} . \varepsilon>0\}
\end{aligned}
$$

Mas, considerando-se ainda uma definição geral para a hiersuperfície, a resposta do meio material pode então ser descrita, de acordo com os regimes predominantes de compressão e de tração, por funções de energia elástica $W_{-}(\varepsilon)$ e $\mathrm{W}_{+}(\varepsilon)$, restritas aos subdomínios $\varepsilon_{-}$e $\varepsilon_{+}$respectivamente, sendo que juntas compõem uma função de energia W $(\varepsilon)$ definida sobre $\varepsilon$. 
De modo mais específico passam a valer as seguintes relações:

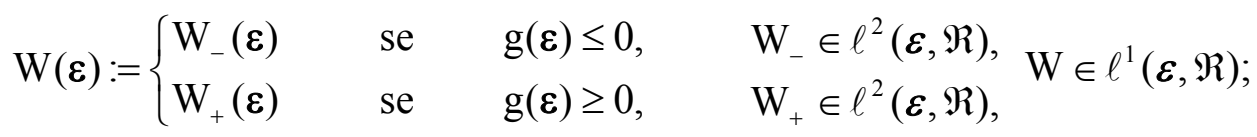

$$
\begin{aligned}
& \sigma(\varepsilon)=\nabla_{\varepsilon} \mathrm{W}(\varepsilon)=\left\{\begin{array}{lll}
\sigma_{-}(\varepsilon)=\nabla_{\varepsilon} \mathrm{W}_{-}(\varepsilon) & \text { se } & \mathrm{g}(\varepsilon) \leq 0 \\
\sigma_{+}(\varepsilon)=\nabla_{\varepsilon} \mathrm{W}_{+}(\varepsilon) & \text { se } & \mathrm{g}(\varepsilon) \geq 0
\end{array}\right. \\
& \mathbf{E}_{0}(\varepsilon)=\left\{\begin{array}{lll}
\mathrm{E}_{0-}(\varepsilon)=\nabla_{\varepsilon}^{2} \mathrm{~W}_{-}(\varepsilon) & \text { se } & \mathrm{g}(\varepsilon)<0 \\
\mathrm{E}_{0+}(\varepsilon)=\nabla_{\varepsilon}^{2} \mathrm{~W}_{+}(\varepsilon) & \text { se } & \mathrm{g}(\varepsilon)>0
\end{array}\right.
\end{aligned}
$$

onde, $\nabla$ é o operador gradiente e,

$$
\begin{aligned}
& \mathrm{W}(0)=\mathrm{W}_{-}(0)=\mathrm{W}_{+}(0)=0 \\
& \sigma(0)=\sigma_{-}(0)=\sigma_{+}(0)=0
\end{aligned}
$$

A continuidade da função de energia elástica (e de seu primeiro gradiente), e a lei tensão-deformação, ao longo da interface, implicam em

$$
\begin{array}{ll}
\mathrm{W}(\varepsilon)=\mathrm{W}_{+}(\varepsilon)=\mathrm{W}_{-}(\varepsilon) & \forall \varepsilon \mid g(\varepsilon)=0 \\
\sigma(\varepsilon)=\sigma_{+}(\varepsilon)=\sigma_{-}(\varepsilon) & \forall \varepsilon \mid g(\varepsilon)=0
\end{array}
$$

Já o tensor de rigidez elástica por hipótese apresenta-se contínuo ao longo da hipersuperfície e descontínuo através dela, isto é, segundo a normal local definida por $\nabla_{\varepsilon} \mathrm{g}$. O salto do tensor pode ser representado por:

$$
\left[\mathbf{E}_{0}(\varepsilon)\right]:=\mathbf{E}_{0+}(\varepsilon)-\mathbf{E}_{0-}(\varepsilon)=\mathrm{s}(\varepsilon) \nabla_{\varepsilon} \mathrm{g}(\varepsilon) \otimes \nabla_{\varepsilon} \mathrm{g}(\varepsilon) \quad \forall \varepsilon \mid g(\varepsilon)=0
$$

onde s ( $\varepsilon$ ) é uma função contínua de valor escalar que mede o valor do salto.

Analogamente, no espaço dual de tensões define-se uma hipersuperfície h $(\sigma)$ e uma interface $\not^{*}$ dada por: 


$$
\not \check{C}^{*}:=\{\sigma \in \wp \mid h(\sigma)=0\}, h(\sigma)=g\left[\varepsilon_{-}(\sigma)\right] \text { ou } g\left[\varepsilon_{+}(\sigma)\right] h \in \ell^{1}(\wp, \mathfrak{R})
$$

As relações duais podem ser escritas como:

$$
\begin{aligned}
& \mathrm{W}^{*}(\sigma):=\left\{\begin{array}{llll}
\mathrm{W}_{-}(\sigma) & \text { se } & \mathrm{h}(\sigma) \leq 0, & \mathrm{~W}_{-}^{*} \in \ell^{2}(\wp, \Re), \\
\mathrm{W}_{+}(\sigma) & \text { se } & \mathrm{h}(\sigma) \geq 0, & \mathrm{~W}_{+}^{*} \in \ell^{2}(\wp, \mathfrak{R}),
\end{array} \mathrm{W}^{*} \in \ell^{1}(\wp, \Re) ;\right. \\
& \varepsilon(\sigma)=\nabla_{\sigma} W^{*}(\sigma)=\left\{\begin{array}{lll}
\varepsilon_{-}(\sigma)=\nabla_{\sigma} W_{-}^{*}(\sigma) & \text { se } & h(\sigma) \leq 0 \\
\varepsilon_{+}(\sigma)=\nabla_{\sigma} W_{+}^{*}(\sigma) & \text { se } & h(\sigma) \geq 0
\end{array}\right. \\
& \mathbf{C}_{0}(\sigma)=\mathbf{E}_{0}^{-1}(\sigma)=\left\{\begin{array}{lll}
\mathrm{C}_{0-}(\sigma)=\nabla_{\sigma}^{2} \mathrm{~W}_{-}^{*}(\sigma) & \text { se } & \mathrm{h}(\sigma)<0 ; \\
\mathrm{C}_{0+}(\sigma)=\nabla_{\sigma}^{2} \mathrm{~W}_{+}^{*}(\sigma) & \text { se } & \mathrm{h}(\sigma)>0 ;
\end{array}\right.
\end{aligned}
$$

As condições de continuidade para as relações duais são similares às equações $(2.25)-(2.27)$, com $\nabla_{\varepsilon} g(\varepsilon)$ substituído por $\nabla_{\sigma} h(\sigma)$ e s $(\varepsilon)$ por

$\mathrm{e}(\sigma)=-\mathrm{s}(\varepsilon) /\left[1+\mathrm{s}(\varepsilon) \nabla_{\sigma} h(\sigma): \nabla_{\varepsilon} g(\varepsilon)\right]$

No caso do hiperplano, o caráter invariante de $g(\varepsilon)$ pode ser explicitado representando-o como uma combinação linear dos invariantes lineares de deformação, $\mathrm{I}_{\mathrm{a}}=\left(\operatorname{tr} \mathbf{A}_{\mathbf{a}} \varepsilon\right)$, isto é: $g(\varepsilon)=\gamma_{\mathrm{a}} \mathbf{I}_{\mathbf{a}}$. Nessas condições, seu gradiente $\nabla_{\varepsilon} g(\varepsilon)$ $=\mathbf{N}$, definindo a normal ao hiperplano, resulta uma combinação linear dos correspondentes tensores $\mathbf{A}_{\mathbf{a}}$. Para exemplificar no caso mais geral, que inclui também a ortotropia, passam a valer as seguintes relações:

$$
\begin{aligned}
& \mathbf{N}=\nabla_{\varepsilon} g=\frac{\partial g}{\partial \mathbf{I}_{\mathrm{a}}} \nabla_{\varepsilon} \mathbf{I}_{\mathbf{a}}=\gamma_{\mathbf{a}} \mathbf{A}_{\mathbf{a}}, \text { onde os coeficientes } \gamma_{\mathrm{a}} \text { são tais que: }\|\mathbf{N}\|=1 \\
& g(\boldsymbol{\varepsilon})=\gamma_{\mathrm{a}} \operatorname{tr}\left(\mathbf{A}_{\mathbf{a}} \varepsilon\right)=\operatorname{tr}\left[\left(\gamma_{\mathrm{a}} \mathbf{A}_{\mathbf{a}}\right) \varepsilon\right]=\mathbf{N} . \varepsilon=0, \quad(\mathrm{a}=1, \mathrm{~d}),
\end{aligned}
$$

Finalmente, as expressões para a função de energia elástica (2.20), para a lei tensão-deformação (2.21) e para o tensor dos módulos elásticos (2.22) são escritas, em analogia com (2.7), (2.8) e (2.9), nas seguintes formas: 


$$
\begin{aligned}
& \mathbf{W}(\mathbf{g}(\varepsilon), \varepsilon)=\frac{\lambda_{\mathrm{ab}}(\mathbf{g}(\varepsilon))}{2} \operatorname{tr}\left(\mathbf{A}_{\mathrm{a}} \varepsilon\right) \operatorname{tr}\left(\mathbf{A}_{\mathrm{b}} \varepsilon\right)+\mu_{\mathrm{a}} \operatorname{tr}\left(\mathbf{A}_{\mathrm{a}} \varepsilon^{2}\right) \quad(\mathrm{a}, \mathrm{b}=1, \mathrm{~d}) \\
& \sigma(\mathbf{g}(\varepsilon), \varepsilon)=\lambda_{\mathrm{ab}}(\mathbf{g}(\varepsilon)) \operatorname{tr}\left(\mathbf{A}_{\mathrm{a}} \varepsilon\right) \mathbf{A}_{\mathrm{b}}+\mu_{\mathrm{a}}\left(\mathbf{A}_{\mathrm{a}} \varepsilon+\varepsilon \mathbf{A}_{\mathrm{a}}^{\mathrm{T}}\right) \\
& \mathbf{E}_{0}(\mathbf{g}(\varepsilon), \varepsilon)=\lambda_{\mathrm{ab}}(\mathbf{g}(\varepsilon))\left[\mathbf{A}_{\mathrm{a}} \otimes \mathbf{A}_{\mathrm{b}}\right]+\mu_{\mathrm{a}}\left[\mathbf{A}_{\mathrm{a}} \otimes \mathbf{I}+\mathbf{I} \otimes \mathbf{A}_{\mathrm{a}}\right]
\end{aligned}
$$

O caráter bimodular implica em que as constantes $\lambda_{\mathrm{ab}}$ que aparecem nas relações (2.35) - (2.37) são funções de g, isto é:

$$
\lambda_{\mathrm{ab}}(\mathrm{g}(\varepsilon))=\lambda_{\mathrm{ba}}:=\left(\begin{array}{lll}
\lambda_{\mathrm{ab}}^{-} & \text {se } & \mathbf{g}(\varepsilon)<0 \\
\lambda_{\mathrm{ab}}^{+} & \text {se } & \mathbf{g}(\varepsilon)>0
\end{array}\right.
$$

E mantendo a mesma analogia,as leis inversas são descritas como:

$$
\begin{aligned}
& \mathrm{W}^{*}(\mathrm{~h}(\sigma), \sigma)=-\frac{\mathrm{v}_{\mathrm{ab}}(\mathrm{h}(\sigma))}{2 \mathrm{E}_{\mathrm{a}}(\mathrm{h}(\sigma))} \operatorname{tr}\left(\mathbf{A}_{\mathrm{a}} \sigma\right) \operatorname{tr}\left(\mathbf{A}_{\mathrm{b}} \sigma\right)+\frac{1+\mathrm{v}_{\mathrm{aa}}(\mathrm{h}(\sigma))}{2 \mathrm{E}_{\mathrm{a}}(\mathrm{h}(\sigma))} \operatorname{tr}\left(\mathbf{A}_{\mathrm{a}} \sigma^{2}\right) \\
& (\mathrm{a}, \mathrm{b}=1, \mathrm{~d}) \\
& \varepsilon(\mathrm{h}(\sigma), \sigma)=-\frac{v_{\mathrm{ab}}(\mathrm{h}(\sigma))}{\mathrm{E}_{\mathrm{a}}(\mathrm{h}(\sigma))} \operatorname{tr}\left(\mathbf{A}_{\mathrm{a}} \sigma\right) \mathbf{A}_{\mathrm{b}}+\frac{1+\mathrm{v}_{\mathrm{aa}}(\mathrm{h}(\sigma))}{2 \mathrm{E}_{\mathrm{a}}(\mathrm{h}(\sigma))}\left(\mathbf{A}_{\mathrm{a}} \sigma+\sigma \mathbf{A}_{\mathrm{a}}^{\mathrm{T}}\right) \\
& \mathbf{C}_{\mathbf{0}}(\mathrm{h}(\sigma), \sigma)=-\frac{\mathrm{v}_{\mathrm{ab}}(\mathrm{h}(\sigma))}{\mathrm{E}_{\mathrm{a}}(\mathrm{h}(\sigma))}\left[\mathbf{A}_{\mathrm{a}} \otimes \mathbf{A}_{\mathrm{b}}\right]+\frac{1+\mathrm{v}_{\mathrm{aa}}(\mathrm{h}(\sigma))}{2 \mathrm{E}_{\mathrm{a}}(\mathrm{h}(\sigma))}\left[\mathbf{A}_{\mathrm{a}} \otimes-\overline{\mathbf{I}}+\mathbf{I} \otimes \mathbf{A}_{\mathrm{a}}\right] \\
& \mathrm{E}_{\mathrm{a}}(\mathrm{h}(\sigma)):=\left\{\begin{array}{lll}
\mathrm{E}_{\mathrm{a}}^{-} & \text {se } & \mathbf{h}(\sigma)<0 \\
\mathrm{E}_{\mathrm{a}}^{+} & \text {se } & \mathbf{h}(\sigma)>0
\end{array}, v_{\mathrm{ab}}(\mathrm{h}(\sigma)):=\left\{\begin{array}{lll}
v_{\mathrm{ab}}^{-} & \text {se } & \mathrm{h}(\sigma)<0 \\
v_{\mathrm{ab}}^{+} & \text {se } & \mathrm{h}(\sigma)>0
\end{array}\right.\right.
\end{aligned}
$$

Vale ressaltar que os coeficientes $\mu_{\mathrm{a}}$ [eq. $\left.(2.35)-(2.37)\right]$ e $\frac{1+\mathrm{v}_{\mathrm{aa}}(\mathrm{h}(\sigma))}{2 \mathrm{E}_{\mathrm{a}}(\mathrm{h}(\sigma))}[$ eq. (2.39) - (2.41)] devem ser iguais em compressão e em tração para que se verifique a condição de salto tangencial nulo no tensor constitutivo [ eq. (2.27)]. 


\subsection{PROPOSTA DE FORMULAÇÃO PARA MEIOS ELÁSTICOS ANISÓTROPOS COM DANIFICAÇÃO E RESPOSTA BIMODULAR}

Esta proposta de formulação visa estender os modelos de elasticidade linear em meios anisótropos bimodulares postulados por CURNIER, HE \& ZYSSET (1995), de modo a se levar em conta a danificação nesses meios.

Propõe-se que os coeficientes $\lambda_{\mathrm{ab}}, \mu_{\mathrm{a}}$ [eq. (2.35) - (2.37)] e $v_{\mathrm{ab}}, v_{\mathrm{aa}}$ e $\mathrm{E}_{\mathrm{a}}$ [eq. (2.39) - (2.41)] passem a ser funções de variáveis de danificação, bem como o próprio hiperplano separador das respostas em tração e em compressão. Contudo, vale ressaltar que as condições de salto tangencial nulo devem também ser obedecidas aqui. Com isso os coeficientes $\mu_{\mathrm{a}}$ e $\frac{1+\mathrm{v}_{\mathrm{aa}}(\mathrm{h}(\sigma))}{2 \mathrm{E}_{\mathrm{a}}(\mathrm{h}(\sigma))}$ devem ser iguais em tração e em compressão.

Com a introdução de processos de danificação na formulação proposta por Curnier, a lei tensão-deformação passa a sofrer a influência das variáveis de dano. Como o critério para a identificação das respostas constitutivas de compressão ou de tração, hiperplano separador g ou h, é função das componentes de tensão ou de deformação, este critério é então influenciado pelas variáveis de dano.

Outra observação pertinente é sobre a introdução de variáveis associadas ao dano, que podem ser interpretadas como taxas de liberação de energia durante o processo de evolução do dano. Tais variáveis podem ser empregadas na definição dos critérios que identificam o início e a evolução da danificação e que são necessários para a complementação da formulação, conforme será discutido mais adiante.

A escolha das variáveis de dano e da classe de anisotropia consideradas num modelo depende do material que se deseja simular. Nesse sentido, é importante observar que a danificação influencia também a forma de variação dos tensores de quarta de ordem $\left[\mathbf{A}_{\mathbf{a}} \otimes \mathbf{A}_{\mathbf{b}}\right]$ e $\left[\mathbf{A}_{\mathbf{a}} \otimes \bar{I}+\mathbf{I} \otimes \mathbf{A}_{\mathbf{a}}\right]$ [eq. (2.37)].

As expressões para a função de energia, para a relação tensão-deformação e para o tensor dos módulos elásticos de rigidez são escritas, em analogia com (2.35), (2.36) e (2.37), nas seguintes formas: 


$$
\begin{aligned}
& \mathrm{W}\left(\mathrm{D}_{\mathrm{i}}, \mathrm{g}(\varepsilon), \varepsilon\right)=\frac{\lambda_{\mathrm{ab}}\left(\mathrm{D}_{\mathrm{i}}, \mathrm{g}(\boldsymbol{\varepsilon})\right)}{2} \operatorname{tr}\left(\mathbf{A}_{\mathrm{a}} \varepsilon\right) \operatorname{tr}\left(\mathbf{A}_{\mathrm{b}} \varepsilon\right)+\mu_{\mathrm{a}}\left(\mathrm{D}_{\mathrm{i}}\right) \operatorname{tr}\left(\mathbf{A}_{\mathrm{a}} \varepsilon^{2}\right) \quad(\mathrm{a}, \mathrm{b}=1, \mathrm{~d}) \\
& \sigma\left(D_{i}, g(\varepsilon), \varepsilon\right)=\lambda_{a b}\left(D_{i}, g(\varepsilon)\right) \operatorname{tr}\left(\mathbf{A}_{\mathbf{a}} \varepsilon\right) \mathbf{A}_{\mathbf{b}}+\mu_{\mathrm{a}}\left(D_{\mathrm{i}}\right)\left(\mathbf{A}_{\mathbf{a}} \varepsilon+\varepsilon \mathbf{A}_{\mathrm{a}}^{\mathrm{T}}\right) \\
& \mathbf{E}\left(\mathrm{D}_{\mathrm{i}}, \mathrm{g}(\boldsymbol{\varepsilon}), \boldsymbol{\varepsilon}\right)=\lambda_{\mathrm{ab}}\left(\mathrm{D}_{\mathrm{i}}, \mathrm{g}(\boldsymbol{\varepsilon})\right)\left[\mathbf{A}_{\mathrm{a}} \otimes \mathbf{A}_{\mathrm{b}}\right]+\mu_{\mathrm{a}}\left(\mathrm{D}_{\mathrm{i}}\right)\left[\mathbf{A}_{\mathrm{a}} \bar{\otimes} \mathbf{I}+\mathbf{I} \otimes \mathbf{A}_{\mathrm{a}}\right]
\end{aligned}
$$

onde o sub-índice i pode assumir valores de 1 até o número de variáveis escalares de dano que os modelos venham a considerar.

As variáveis associadas ao dano são obtidas da variação da função de energia (2.43) em relação ao incremento dos processos de danificação. Portanto,

$$
\begin{aligned}
& \mathbf{Y}\left(\mathrm{D}_{\mathrm{i}}, \mathrm{g}\left(\varepsilon, \mathrm{D}_{\mathrm{i}}\right), \varepsilon\right)=\nabla_{\mathrm{D}} \mathrm{W}=\frac{\partial \mathrm{W}}{\partial \mathrm{D}_{\mathrm{i}}}=-\frac{1}{2} \frac{\partial \lambda_{\mathrm{ab}}\left(\mathrm{D}_{\mathrm{i}}, \mathrm{g}\left(\varepsilon, \mathrm{D}_{\mathrm{i}}\right)\right)}{\partial \mathrm{D}_{\mathrm{i}}} \operatorname{tr}\left(\mathbf{A}_{\mathrm{a}} \varepsilon\right) \operatorname{tr}\left(\mathbf{A}_{\mathrm{b}} \varepsilon\right) \\
& -\frac{\partial \mu_{\mathrm{a}}\left(\mathrm{D}_{\mathrm{i}}\right)}{\partial \mathrm{D}_{\mathrm{i}}} \operatorname{tr}\left(\mathbf{A}_{\mathrm{a}} \varepsilon^{2}\right)
\end{aligned}
$$

Como se observa, o caráter bimodular implica que os coeficientes $\lambda_{\mathrm{ab}}$ são funções de $\mathrm{g}\left(\varepsilon, \mathrm{D}_{\mathrm{i}}\right)$, isto é:

$$
\lambda_{\mathrm{ab}}\left(\mathrm{D}_{\mathrm{i}}, \mathrm{g}\left(\varepsilon, \mathrm{D}_{\mathrm{i}}\right)\right)=\lambda_{\mathrm{ba}}:=\left(\begin{array}{lll}
\lambda_{\mathrm{ab}}^{-}\left(\mathrm{D}_{\mathrm{i}}\right) & \text { se } & \mathrm{g}\left(\varepsilon, \mathrm{D}_{\mathrm{i}}\right)<0 \\
\lambda_{\mathrm{ab}}^{+}\left(\mathrm{D}_{\mathrm{i}}\right) & \text { se } & \mathrm{g}\left(\varepsilon, \mathrm{D}_{\mathrm{i}}\right)>0
\end{array}\right.
$$

Analogamente, as leis inversas podem ser escritas como:

$$
\begin{aligned}
\mathrm{W}^{*}\left(\mathrm{D}_{\mathrm{i}}, \mathrm{h}\left(\sigma, \mathrm{D}_{\mathrm{i}}\right), \sigma\right)= & -\frac{\mathrm{v}_{\mathrm{ab}}\left(\mathrm{D}_{\mathrm{i}}, \mathrm{h}\left(\sigma, \mathrm{D}_{\mathrm{i}}\right)\right)}{2 \mathrm{E}_{\mathrm{a}}\left(\mathrm{D}_{\mathrm{i}}, \mathrm{h}\left(\sigma, \mathrm{D}_{\mathrm{i}}\right)\right)} \operatorname{tr}\left(\mathbf{A}_{\mathrm{a}} \sigma\right) \operatorname{tr}\left(\mathbf{A}_{\mathrm{b}} \sigma\right) \\
& +\frac{1+\mathrm{v}_{\mathrm{aa}}\left(\mathrm{D}_{\mathrm{i}}, \mathrm{h}\left(\sigma, \mathrm{D}_{\mathrm{i}}\right)\right)}{2 \mathrm{E}_{\mathrm{a}}\left(\mathrm{D}_{\mathrm{i}}, \mathrm{h}\left(\sigma, \mathrm{D}_{\mathrm{i}}\right)\right)} \operatorname{tr}\left(\mathbf{A}_{\mathrm{a}} \sigma^{2}\right) \quad(\mathrm{a}, \mathrm{b}=1, \mathrm{~d}) \\
\varepsilon\left(\mathrm{D}_{\mathrm{i}}, \mathbf{h}\left(\sigma, \mathrm{D}_{\mathrm{i}}\right), \sigma\right)=- & \frac{v_{\mathrm{ab}}\left(\mathrm{D}_{\mathrm{i}}, \mathrm{h}\left(\sigma, \mathrm{D}_{\mathrm{i}}\right)\right)}{\mathrm{E}_{\mathrm{a}}\left(\mathrm{D}_{\mathrm{i}}, \mathrm{h}\left(\sigma, \mathrm{D}_{\mathrm{i}}\right)\right)} \operatorname{tr}\left(\mathbf{A}_{\mathrm{a}} \sigma\right) \mathbf{A}_{\mathbf{b}} \\
& +\frac{1+\mathrm{v}_{\mathrm{aa}}\left(\mathrm{D}_{\mathrm{i}}, \mathrm{h}\left(\sigma, \mathrm{D}_{\mathrm{i}}\right)\right)}{2 \mathrm{E}_{\mathrm{a}}\left(\mathrm{D}_{\mathrm{i}}, \mathrm{h}\left(\sigma, \mathrm{D}_{\mathrm{i}}\right)\right)}\left(\mathbf{A}_{\mathrm{a}} \sigma+\sigma \mathbf{A}_{\mathrm{a}}^{\mathbf{T}}\right)
\end{aligned}
$$




$$
\begin{aligned}
& \mathbf{C}\left(\mathrm{D}_{\mathrm{i}}, \mathbf{h}\left(\sigma, \mathrm{D}_{\mathrm{i}}\right), \sigma\right)=-\frac{v_{\mathrm{ab}}\left(\mathrm{D}_{\mathrm{i}}, \mathrm{h}\left(\sigma, \mathrm{D}_{\mathrm{i}}\right)\right)}{\mathrm{E}_{\mathrm{a}}\left(\mathrm{D}_{\mathrm{i}}, \mathrm{h}\left(\sigma, \mathrm{D}_{\mathrm{i}}\right)\right)}\left[\mathbf{A}_{\mathrm{a}} \otimes \mathbf{A}_{\mathbf{b}}\right] \\
& +\frac{1+v_{\mathrm{aa}}\left(\mathrm{D}_{\mathrm{i}}, \mathrm{h}\left(\sigma, \mathrm{D}_{\mathrm{i}}\right)\right)}{2 \mathrm{E}_{\mathrm{a}}\left(\mathrm{D}_{\mathrm{i}}, \mathrm{h}\left(\sigma, \mathrm{D}_{\mathrm{i}}\right)\right)}\left[\mathbf{A}_{\mathrm{a}} \underset{-}{\otimes} \mathbf{I}+\mathbf{I} \otimes-\mathbf{A}_{\mathrm{a}}\right] \\
& \mathbf{Y}\left(\mathrm{D}_{\mathrm{i}}, \mathrm{h}\left(\sigma, \mathrm{D}_{\mathrm{i}}\right), \sigma\right)=\nabla_{\mathrm{D}} \mathrm{W}^{*}=\frac{\partial \mathrm{W}^{*}}{\partial \mathrm{D}_{\mathrm{i}}}=-\frac{1}{2} \frac{\partial \lambda_{\mathrm{ab}}^{*}\left(\mathrm{D}_{\mathrm{i}}, \mathrm{h}\left(\sigma, \mathrm{D}_{\mathrm{i}}\right)\right)}{\partial \mathrm{D}_{\mathrm{i}}} \operatorname{tr}\left(\mathbf{A}_{\mathrm{a}} \sigma\right) \operatorname{tr}\left(\mathbf{A}_{\mathbf{b}} \sigma\right) \\
& -\frac{\partial \mu_{\mathrm{a}}^{*}\left(\mathrm{D}_{\mathrm{i}}\right)}{\partial \mathrm{D}_{\mathrm{i}}} \operatorname{tr}\left(\mathbf{A}_{\mathbf{a}} \sigma^{2}\right) \\
& E_{a}\left(D_{i}, h\left(\sigma, D_{i}\right)\right):=\left\{\begin{array}{ll}
E_{a}^{-}\left(D_{i}\right) \text { se } & h\left(\sigma, D_{i}\right)<0 \\
E_{a}^{+}\left(D_{i}\right) \text { se } & h\left(\sigma, D_{i}\right)>0
\end{array}\right. \text {, } \\
& v_{a b}\left(D_{i}, h\left(\sigma, D_{i}\right)\right):=\left\{\begin{array}{lll}
v_{a b}^{-}\left(D_{i}\right) & \text { se } & h\left(\sigma, D_{i}\right)<0 \\
v_{a b}^{+}\left(D_{i}\right) & \text { se } & h\left(\sigma, D_{i}\right)>0
\end{array}\right.
\end{aligned}
$$

onde $\lambda_{a b}^{*}\left(D_{i}, h\left(\sigma, D_{i}\right)\right)=-\frac{v_{a b}\left(D_{i}, h\left(\sigma, D_{i}\right)\right)}{E_{a}\left(D_{i}, h\left(\sigma, D_{i}\right)\right)}$ e $\mu_{a}^{*}\left(D_{i}\right)=\frac{1+v_{a a}\left(D_{i}, h\left(\sigma, D_{i}\right)\right)}{2 E_{a}\left(D_{i}, h\left(\sigma, D_{i}\right)\right)}$ são utilizados daqui em diante para simplificar a escrita das expressões.

Observa-se que a formulação proposta pode ser empregada tanto para materiais elásticos danificados possuindo anisotropia e caráter bimodular iniciais, como para materiais elásticos danificados com anisotropia e bimodularidade induzidas pelo dano. Neste último caso, a inserção das variáveis de dano na formulação é feita de modo criterioso para gerar um certo grau de anisotropia do material, assim como para induzir o surgimento do caráter bimodular. Um exemplo dessa possibilidade é abordado a seguir.

\subsubsection{Caso Particular de Isotropia Transversal Induzida pela Danificação}

Neste item, o material comporta-se inicialmente como um meio isótropo com iguais rigidezes em tração e em compressão; com o surgimento e a evolução da danificação, o material passa a apresentar um comportamento bimodular com anisotropia. Trata-se de um caso de interesse porque será adotado mais adiante na formulação de um modelo de dano para o concreto, pois como foi comentado no item 
2.2 deste capítulo e nas observações experimentais do capítulo anterior, o concreto possui as características descritas acima.

Admitindo-se, pois, uma situação genérica em que o meio já apresente um certo nível de dano, que gerou um estado de isotropia transversal, passam a valer as seguintes relações para o modelo:

$$
\begin{aligned}
\mathrm{W}\left(\mathrm{D}_{\mathrm{i}}, \mathrm{g}\left(\varepsilon, \mathrm{D}_{\mathrm{i}}\right), \varepsilon\right) & =\frac{\lambda_{11}}{2} \operatorname{tr}^{2}(\varepsilon)+\mu_{1} \operatorname{tr}\left(\varepsilon^{2}\right)-\frac{\lambda_{22}\left(\mathrm{D}_{\mathrm{i}}, \mathrm{g}\left(\varepsilon, \mathrm{D}_{\mathrm{i}}\right)\right)}{2} \operatorname{tr}^{2}(\mathrm{~A} \varepsilon) \\
& -\lambda_{12}\left(\mathrm{D}_{\mathrm{i}}, \mathrm{g}\left(\varepsilon, \mathrm{D}_{\mathrm{i}}\right)\right) \operatorname{tr}(\varepsilon) \operatorname{tr}(\mathrm{A} \varepsilon)-\mu_{2}\left(\mathrm{D}_{\mathrm{i}}\right) \operatorname{tr}\left(\mathbf{A} \varepsilon^{2}\right) \\
\sigma\left(\mathrm{D}_{\mathrm{i}}, \mathrm{g}\left(\varepsilon, \mathrm{D}_{\mathrm{i}}\right), \varepsilon\right) & =\lambda_{11} \operatorname{tr}(\varepsilon) \mathbf{I}+2 \mu_{1} \varepsilon-\lambda_{22}\left(\mathrm{D}_{\mathrm{i}}, \mathrm{g}\left(\varepsilon, \mathrm{D}_{\mathrm{i}}\right)\right) \operatorname{tr}(\mathrm{A} \varepsilon) \mathbf{A} \\
& -\lambda_{12}\left(\mathrm{D}_{\mathrm{i}}, \mathrm{g}\left(\varepsilon, \mathrm{D}_{\mathrm{i}}\right)\right)[\operatorname{tr}(\varepsilon) \mathbf{A}+\operatorname{tr}(\mathbf{A} \varepsilon) \mathbf{I}]-\mu_{2}\left(\mathrm{D}_{\mathrm{i}}\right)\left[\mathbf{A} \varepsilon+\varepsilon \mathbf{A}^{\mathrm{T}}\right]
\end{aligned}
$$

$\mathbf{E}\left(\mathrm{D}_{\mathrm{i}}, \mathrm{g}\left(\varepsilon, \mathrm{D}_{\mathrm{i}}\right), \varepsilon\right)=\mathbf{E}_{\mathbf{0}}-\lambda_{22}\left(\mathrm{D}_{\mathrm{i}}, \mathrm{g}\left(\boldsymbol{\varepsilon}, \mathrm{D}_{\mathrm{i}}\right)\right)[\mathbf{A} \otimes \mathbf{A}]-\lambda_{12}\left(\mathrm{D}_{\mathrm{i}}, \mathrm{g}\left(\varepsilon, \mathrm{D}_{\mathrm{i}}\right)\right)[\mathbf{A} \otimes \mathbf{I}+\mathbf{I} \otimes \mathbf{A}]$

$$
-\mu_{2}\left(\mathrm{D}_{\mathrm{i}}\right)[\mathbf{A} \stackrel{-}{\otimes} \mathbf{I}+\mathbf{I} \stackrel{-}{\otimes} \mathbf{A}]
$$

$\mathbf{Y}\left(\mathrm{D}_{\mathrm{i}}, \mathrm{g}\left(\varepsilon, \mathrm{D}_{\mathrm{i}}\right), \boldsymbol{\varepsilon}\right)=\nabla_{\mathrm{D}} \mathrm{W}=\frac{\partial \mathrm{W}}{\partial \mathrm{D}_{\mathrm{i}}}=-\frac{1}{2} \frac{\partial \lambda_{22}\left(\mathrm{D}_{\mathrm{i}}, \mathrm{g}\left(\varepsilon, \mathrm{D}_{\mathrm{i}}\right)\right)}{\partial \mathrm{D}_{\mathrm{i}}} \operatorname{tr}^{2}(\mathbf{A} \boldsymbol{\varepsilon})$

$$
-\frac{\partial \lambda_{12}\left(D_{i}, g\left(\varepsilon, D_{i}\right)\right)}{\partial D_{i}} \operatorname{tr}(\varepsilon) \operatorname{tr}(A \varepsilon)-\frac{\partial \mu_{2}\left(D_{i}\right)}{\partial D_{i}} \operatorname{tr}\left(A \varepsilon^{2}\right)
$$

sendo $\lambda_{11}=\lambda_{0}$ e $\mu_{1}=\mu_{0}$ as constantes de Lamè e $\mathbf{E}_{\mathbf{0}}$ o tensor de rigidez elástica isótropo.

O caráter bimodular é levado em conta pelas seguintes condições:

$$
\begin{aligned}
& \lambda_{12}\left(D_{i}, g\left(\varepsilon, D_{i}\right)\right):=\left(\begin{array}{ll}
\lambda_{12}^{-}\left(D_{i}\right) \text { se } & g\left(\varepsilon, D_{i}\right)<0 \\
\lambda_{12}^{+}\left(D_{i}\right) \text { se } & g\left(\varepsilon, D_{i}\right)>0
\end{array}\right. \\
& \lambda_{22}\left(D_{i}, g\left(\varepsilon, D_{i}\right)\right):=\left(\begin{array}{llr}
\lambda_{22}^{-}\left(D_{i}\right) & \text { se } & g\left(\varepsilon, D_{i}\right)<0 \\
\lambda_{22}^{+}\left(D_{i}\right) & \text { se } & g\left(\varepsilon, D_{i}\right)>0
\end{array}\right.
\end{aligned}
$$

Observa-se ainda que para valores nulos das variáveis de dano todos os coeficientes dependentes delas se anulam e o material tem um comportamento de 
meio isótropo. Além disso, nessas condições o meio exibe uma característica unimodular.

Por outro lado, as leis duais são dadas por:

$$
\begin{aligned}
& \mathrm{W}^{*}\left(\mathrm{D}_{\mathrm{i}}, \mathrm{h}\left(\sigma, \mathrm{D}_{\mathrm{i}}\right), \sigma\right)=-\frac{v_{11}}{2 \mathrm{E}_{1}} \operatorname{tr}^{2}(\sigma)+\frac{1+\mathrm{v}_{11}}{2 \mathrm{E}_{1}} \operatorname{tr}\left(\sigma^{2}\right)-\frac{\lambda_{22}^{*}\left(\mathrm{D}_{\mathrm{i}}, \mathrm{h}\left(\sigma, \mathrm{D}_{\mathrm{i}}\right)\right)}{2} \operatorname{tr}^{2}(\mathrm{~A} \sigma) \\
& -\lambda_{12}^{*}\left(D_{i}, h\left(\sigma, D_{i}\right)\right) \operatorname{tr}(\sigma) \operatorname{tr}(A \sigma)-\mu_{2}^{*}\left(D_{i}\right) \operatorname{tr}\left(A \sigma^{2}\right) \\
& \varepsilon\left(\mathrm{D}_{\mathrm{i}}, \mathrm{h}\left(\sigma, \mathrm{D}_{\mathrm{i}}\right), \sigma\right)=-\frac{v_{11}}{\mathrm{E}_{1}} \operatorname{tr}(\sigma) \mathbf{I}+\frac{1+v_{11}}{\mathrm{E}_{1}} \sigma-\lambda_{22}^{*}\left(\mathrm{D}_{\mathrm{i}}, \mathrm{h}\left(\sigma, \mathrm{D}_{\mathrm{i}}\right)\right) \operatorname{tr}(\mathrm{A} \sigma) \mathbf{A} \\
& -\lambda_{12}^{*}\left(D_{i}, h\left(\sigma, D_{i}\right)\right)[\operatorname{tr}(\sigma) \mathbf{A}+\operatorname{tr}(\mathbf{A} \sigma) \mathbf{I}]-\mu^{*}{ }_{2}\left(D_{i}\right)\left[\mathbf{A} \sigma+\sigma \mathbf{A}^{\mathbf{T}}\right]
\end{aligned}
$$

$\mathbf{C}\left(\mathrm{D}_{\mathrm{i}}, \mathrm{h}\left(\sigma, \mathrm{D}_{\mathrm{i}}\right), \sigma\right)=\mathbf{C}_{\mathbf{0}}-\lambda_{22}^{*}\left(\mathrm{D}_{\mathrm{i}}, \mathrm{h}\left(\sigma, \mathrm{D}_{\mathrm{i}}\right)\right)[\mathbf{A} \otimes \mathbf{A}]-\lambda_{12}^{*}\left(\mathrm{D}_{\mathrm{i}}, \mathrm{h}\left(\sigma, \mathrm{D}_{\mathrm{i}}\right)\right)[\mathbf{A} \otimes \mathbf{I}+\mathbf{I} \otimes \mathbf{A}]$

$$
-\mu_{2}^{*}\left(\mathrm{D}_{\mathrm{i}}\right)[\mathbf{A} \underset{-}{\otimes} \mathbf{I}+\mathbf{I} \underset{-}{\otimes} \mathbf{A}]
$$

$\mathbf{Y}\left(\mathrm{D}_{\mathrm{i}}, \mathrm{h}\left(\sigma, \mathrm{D}_{\mathrm{i}}\right), \sigma\right)=\nabla_{\mathrm{D}} \mathrm{W}^{*}=\frac{\partial \mathrm{W}^{*}}{\partial \mathrm{D}_{\mathrm{i}}}=-\frac{1}{2} \frac{\partial \lambda_{22}^{*}\left(\mathrm{D}_{\mathrm{i}}, \mathrm{h}\left(\sigma, \mathrm{D}_{\mathrm{i}}\right)\right)}{\partial \mathrm{D}_{\mathrm{i}}} \operatorname{tr}^{2}(\mathbf{A} \sigma)$

$$
-\frac{\partial \lambda_{12}^{*}\left(\mathrm{D}_{\mathrm{i}}, \mathrm{h}\left(\sigma, \mathrm{D}_{\mathrm{i}}\right)\right)}{\partial \mathrm{D}_{\mathrm{i}}} \operatorname{tr}(\sigma) \operatorname{tr}(\mathrm{A} \sigma)-\frac{\partial \mu_{2}^{*}\left(\mathrm{D}_{\mathrm{i}}\right)}{\partial \mathrm{D}_{\mathrm{i}}} \operatorname{tr}\left(\mathbf{A} \sigma^{2}\right)
$$

Nas relações acima $\mathbf{C}_{\mathbf{0}}$ é o tensor de flexibilidade elástica com isotropia, $\mathrm{E}_{1}=$ $\mathrm{E}_{0}$ e $v_{11}=v_{0}$ são o módulo de elasticidade e o coeficiente de Poisson do material íntegro, respectivamente.

Mais uma vez o caráter bimodular das relações (2.59)-(2.62) é expresso por:

$$
\begin{aligned}
& \lambda_{12}^{*}\left(D_{i}, h\left(\sigma, D_{i}\right)\right):=\left(\begin{array}{lll}
\lambda_{12}^{-*}\left(D_{i}\right) & \text { se } & h\left(\sigma, D_{i}\right)<0 \\
\lambda_{12}^{+*}\left(D_{i}\right) & \text { se } & h\left(\sigma, D_{i}\right)>0
\end{array}\right. \\
& \lambda_{22}^{*}\left(D_{i}, h\left(\sigma, D_{i}\right)\right):=\left(\begin{array}{lll}
\lambda_{22}^{-*}\left(D_{i}\right) & \text { se } & h\left(\sigma, D_{i}\right)<0 \\
\lambda_{22}^{+*}\left(D_{i}\right) & \text { se } & h\left(\sigma, D_{i}\right)>0
\end{array}\right.
\end{aligned}
$$




\subsection{MODELOS DE DANO PARA O CONCRETO}

\subsubsection{Introdução}

O objetivo principal deste item é destacar a estrutura dos tensores constitutivos presentes em modelos elásticos com dano para o concreto propostos na bibliografia. Dentro desse contexto, alguns modelos isótropos de dano escalar são abordados, incluindo-se, também, um caso de dano tensorial. Em seguida, comentase o exemplo de um modelo com anisotropia induzida pelo dano.

\subsubsection{Modelos Isótropos}

Os materiais isótropos possuem infinitos planos e eixos de simetria de resposta, de tal modo que, localmente, as propriedades são as mesmas em todas as direções. Na medida em que as classes de simetria, incluindo-se aí planos e eixos, são em número finito, diferentes níveis de anisotropia se apresentam. A danificação é fator gerador de anisotropia.

Nos chamados modelos de dano isótropos o número de direções ou planos iniciais de simetria do material não se altera, isto é, se o meio é inicialmente isótropo ou anisótropo com algum grau, essas características se preservam com a danificação.

No sentido de representar matematicamente a questão da anisotropia induzida pela danificação, considera-se que o tensor constitutivo de rigidez atual $\mathbf{E}$ possa ser expresso, de modo geral, como a soma do tensor inicial $\mathbf{E}_{\mathbf{0}}$ mais uma parcela dependente do valor atual de dano [RIZZI (1995)]. Essa mesma idéia pode ser estendida para o tensor de flexibilidade C. Quando essa parcela for colocada na forma de uma somatória de funções de valor escalar de variáveis escalares de dano multiplicando tensores constitutivos de quarta ordem, o modelo resultante é dito, genericamente, modelo de dano escalar. Formas aditivas simples para modelos de dano escalar podem ser representadas por exemplo como:

$$
\mathbf{E}=\mathbf{E}_{\mathbf{0}}+\lambda[\mathrm{D}] \overline{\mathbf{M}} \quad \mathrm{e} \quad \mathbf{C}=\mathbf{C}_{\mathbf{0}}+\lambda[\mathrm{D}] \mathbf{M}
$$


onde $\overline{\mathbf{M}}$ e $\mathbf{M}$ são tensores constantes de quarta ordem e $\lambda[D]$ é uma função de valor escalar de uma variável escalar de dano. Supondo-se que o material seja inicialmente isótropo, pode-se preservar a isotropia, ou criar um efeito de anisotropia sobre os tensores constitutivos dependendo das definições de $\overline{\mathbf{M}}$ e $\mathbf{M}$.

A forma mais simples de um modelo de dano escalar é obtida imediatamente adotando-se $\overline{\mathbf{M}}=-\mathbf{E}_{\mathbf{0}}$ e $\lambda[\mathrm{D}]=\mathrm{D}$, ou, em relação à flexibilidade, adotando-se $\mathbf{M}=\mathbf{C}_{\mathbf{0}}$ e $\lambda[D]=D /(1-D)$. Este é o caso do modelo para o concreto proposto por MAZARS (1984), um modelo com uma única variável escalar de dano. Explicitando-se a equação (2.65), para o tensor de flexibilidade pode-se obter a forma de representação abaixo:

$$
\begin{aligned}
& \mathbf{C}=\mathbf{C}_{\mathbf{0}}+\lambda[\mathrm{D}] \mathbf{M}=-\frac{v_{0}}{\mathrm{E}_{0}} \mathbf{I} \otimes \mathbf{I}+\frac{1+v_{0}}{\mathrm{E}_{0}} \mathbf{I I}+\frac{\mathrm{D}}{(1-\mathrm{D})}\left[-\frac{v_{0}}{\mathrm{E}_{0}} \mathbf{I} \otimes \mathbf{I}+\frac{1+v_{0}}{\mathrm{E}_{0}} \mathbf{I I}\right] \\
& \mathbf{C}=-\frac{v_{0}}{\mathrm{E}_{0}} \frac{1}{1-\mathrm{D}} \mathbf{I} \otimes \mathbf{I}+\frac{1}{1-\mathrm{D}} \frac{1+v_{0}}{\mathrm{E}_{0}} \mathbf{I I}
\end{aligned}
$$

Comparando-se a equação acima com uma expressão geral para o tensor de flexibilidade danificada $\left(\mathbf{C}=-\frac{v}{E} \mathbf{I} \otimes \mathbf{I}+\frac{1+v}{E} \mathbf{I I}\right)$, obtém-se o seguinte sistema de equações com incógnitas E e v,

$$
\left\{\begin{array}{l}
-\frac{v}{E}=-\frac{v_{0}}{E_{0}} \frac{1}{1-D} \\
\frac{1+v}{E}=\frac{1}{1-D} \frac{1+v_{0}}{E_{0}}
\end{array}\right.
$$

Resolvendo-se o sistema, resulta:

$$
\mathrm{E}=\mathrm{E}_{0}(1-\mathrm{D}) \quad ; \quad v=v_{0}
$$

Portanto, com esse tipo de formulação o módulo de elasticidade diminui com o acréscimo da danificação e o coeficiente de Poisson permanece inalterado durante todo o processo. Nas figuras 2.2 e 2.3, ilustram-se as variações do módulo de elasticidade e de Poisson com o dano, comparando-se também os comportamentos de outros modelos que serão comentados mais adiante neste item. 


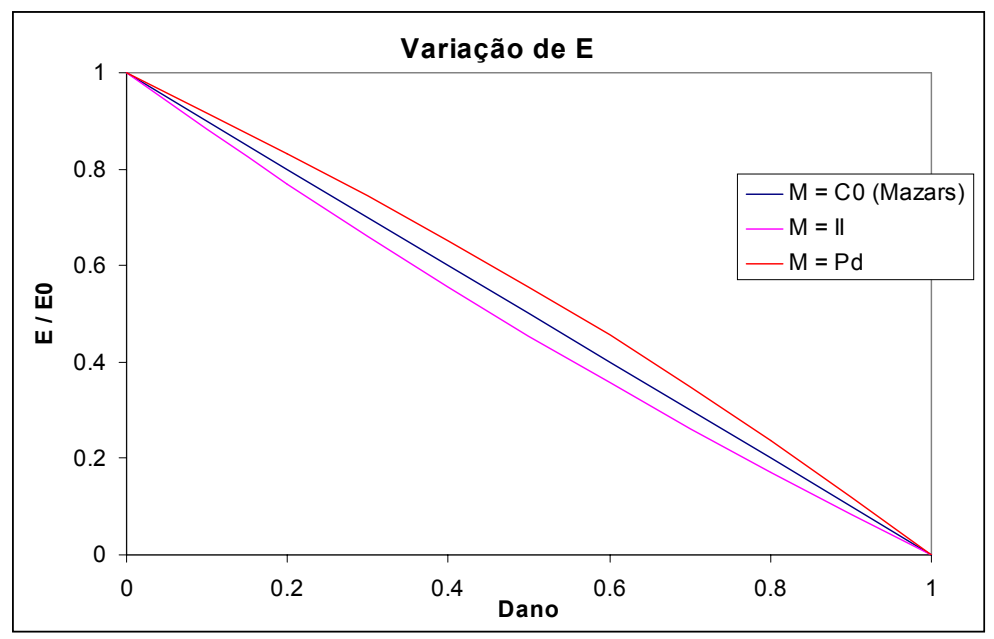

Figura 2.2 - Modelo de dano escalar: módulo de Young em função da variável de dano D.

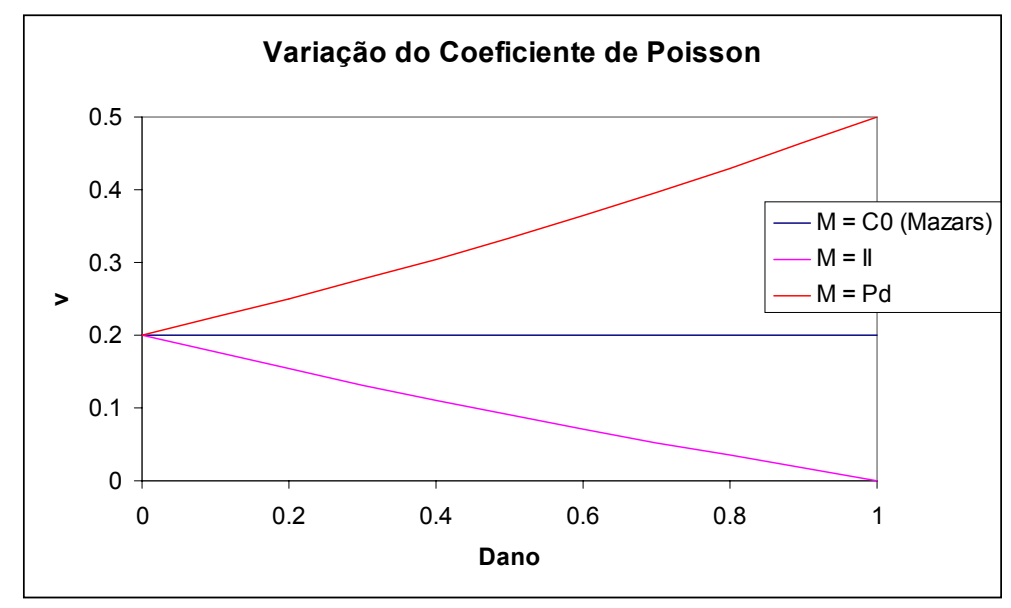

Figura 2.3 - Modelo de dano escalar:

coeficiente de Poisson em função da variável de dano $\mathrm{D}\left(v_{0}=0,2\right)$.

Outras formas de dano escalar são obtidas atribuindo-se outras definições ao tensor M. Nas duas possibilidades descritas a seguir a lei para $\lambda[D]$ é escolhida de modo a penalizar o módulo de elasticidade transversal $\mu=G=(1-D) G_{0}$. Assim, escolhe-se $\lambda[D]=D / 2 \mu_{0}$ (1-D), com $\mu_{0}=\frac{E_{0}}{2\left(1+v_{0}\right)}$ implicando em $\lambda[\mathrm{D}]=\frac{\mathrm{D}\left(1+\mathrm{v}_{0}\right)}{\mathrm{E}_{0}(1-\mathrm{D})}$.

Num primeiro caso adota-se $\mathbf{M}=$ II. Utilizando-se a equação (2.65) para o tensor de flexibilidade, tem-se 


$$
\begin{aligned}
& \mathbf{C}=\mathbf{C}_{\mathbf{0}}+\lambda \mathbf{M}=-\frac{v_{0}}{\mathrm{E}_{0}} \mathbf{I} \otimes \mathbf{I}+\frac{1+v_{0}}{\mathrm{E}_{0}} \mathbf{I I}+\frac{\mathrm{D}\left(1+v_{0}\right)}{\mathrm{E}_{0}(1-\mathrm{D})} \mathbf{I} \mathbf{I} \\
& \mathbf{C}=-\frac{v_{0}}{\mathrm{E}_{0}} \mathbf{I} \otimes \mathbf{I}+\frac{1}{1-\mathrm{D}} \frac{1+v_{0}}{\mathrm{E}_{0}} \mathbf{I I}
\end{aligned}
$$

Resultando, pelo confronto com o tensor geral de flexibilidade, nas seguintes formas para $\mathrm{E}$ e $\mathrm{v}$ :

$$
\mathrm{E}=\frac{(1-\mathrm{D}) \mathrm{E}_{0}}{1+v_{0} \mathrm{D}} \quad ; \quad v=\frac{(1-\mathrm{D}) v_{0}}{1+v_{0} \mathrm{D}}
$$

Neste caso, o módulo de elasticidade e o coeficiente de Poisson diminuem de modo não linear com o aumento da danificação (figuras 2.2 e 2.3), sendo que o coeficiente de Poisson, em particular, tende a um valor nulo. Como exemplo de um modelo que apresenta essas características, pode-se citar uma simplificação de um modelo de degradação da rigidez elástica proposto por ORTIZ (1985) para o comportamento da argamassa.

Uma segunda possibilidade interessante é assumir $\mathbf{M}=\mathbf{P}_{\mathbf{d}}$, sendo $\mathbf{P}_{\mathbf{d}} \mathbf{O}$ operador de projeção desviadora $\left(\mathbf{P}_{\mathbf{d}}=\mathbf{I I}-\mathbf{P}_{\mathbf{v}}=\mathbf{I I}-\mathbf{I} \otimes \mathbf{I} / 3\right)$.

$$
\mathbf{C}=\mathbf{C}_{\mathbf{0}}+\lambda \mathbf{M}=\mathbf{P}_{\mathbf{v}} \frac{1}{3 \mathrm{~K}_{0}}+\mathbf{P}_{\mathbf{d}} \frac{1}{2 \mathrm{G}_{0}}+\lambda \mathbf{M}
$$

onde $\mathrm{K}_{0}$ é o módulo volumétrico $\left(3 \mathrm{~K}_{0}=\mathrm{E}_{0} /\left(1-2 \mathrm{v}_{0}\right)\right)$ e $\mathrm{G}_{0}$ é o módulo de elasticidade transversal $\left(2 \mathrm{G}_{0}=\mathrm{E}_{0} /\left(1+v_{0}\right)\right.$ ). Substituindo-se os valores de $3 \mathrm{~K}_{0}$, $2 \mathrm{G}_{0}, \mathbf{P}_{\mathbf{v}}, \mathbf{P}_{\mathbf{d}}$ e $\lambda[\mathrm{D}]$ na equação anterior, tem-se:

$$
\begin{aligned}
& \mathbf{C}=\frac{\left(1-2 v_{0}\right)}{\mathrm{E}_{0}} \frac{\mathbf{I} \otimes \mathbf{I}}{3}+\frac{1+v_{0}}{\mathrm{E}_{0}}\left(\mathbf{I I}-\frac{\mathbf{I} \otimes \mathbf{I}}{3}\right)+\frac{\mathrm{D}\left(1+v_{0}\right)}{\mathrm{E}_{0}(1-\mathrm{D})}\left(\mathbf{I I}-\frac{\mathbf{I} \otimes \mathbf{I}}{3}\right) \\
& \mathbf{C}=\frac{\left(1-2 v_{0}\right)}{\mathrm{E}_{0}} \frac{\mathbf{I} \otimes \mathbf{I}}{3}+\frac{1+v_{0}}{\mathrm{E}_{0}(1-\mathrm{D})}\left(\mathbf{I I}-\frac{\mathbf{I} \otimes \mathbf{I}}{3}\right)
\end{aligned}
$$

Resultando, pelo confronto com o tensor geral de flexibilidade, nas seguintes expressões:

$$
\mathrm{E}=\frac{3(1-\mathrm{D}) \mathrm{E}_{0}}{3-\left(1-2 v_{0}\right) \mathrm{D}} \quad ; \quad v=\frac{3 v_{0}+\left(1-2 v_{0}\right) \mathrm{D}}{3-\left(1-2 v_{0}\right) \mathrm{D}}
$$


A solução fornece não só uma degradação não-linear do módulo de elasticidade mas também do coeficiente de Poisson com o aumento da danificação como mostrado nas figuras 2.2 e 2.3. Nesse caso, apenas o módulo de cisalhamento é danificado enquanto que o volumétrico permanece constante.

Nesse tipo de formulação com o operador de projeção desviadora enquadra-se o modelo proposto por COMI (2000) para o comportamento em compressão do concreto.

Por outro lado, retomando-se a representação (1.37) do capítulo anterior $\left(\mathbf{E}=(\mathbf{I I}-\mathbf{D}) \mathbf{E}_{0}\right)$ e empregando-se a estrutura indicada nas relações (2.65), um tensor de dano isótropo mais geral pode ser escrito por meio de duas funções escalares independentes $\lambda_{1}\left[D_{1}\right]$ e $\lambda_{2}\left[D_{2}\right]$ no seguinte modo:

$\mathbf{D}=\lambda_{1}\left[\mathrm{D}_{1}\right](\mathbf{I} \otimes \mathbf{I})+\lambda_{2}\left[\mathrm{D}_{2}\right] \mathbf{I I}$

Baseando-se nessa forma, um tensor de dano isótropo de quarta ordem é relatado por CAUVIN \& TESTA (1999).

É importante ressaltar que no contexto apresentado, modelo de dano escalar e modelo de dano isótropo não são sinônimos.

Ainda com relação à nomenclatura, vale novamente observar que quando se faz referência a um modelo de dano isótropo, entende-se que este modelo preserva o numero de planos e direções internas iniciais de simetria do material. Já um modelo de dano anisótropo tem a capacidade de introduzir alterações sobre o número de planos e direções iniciais de simetria, particularmente se o meio é inicialmente isótropo.

\subsubsection{Modelo com Anisotropia Induzida pelo Dano}

Como exemplo de modelo anisótropo de dano, apresenta-se no que segue, resumidamente, o modelo de dano com isotropia transversal induzida proposto por CAUVIN \& TESTA (1999). O modelo tem a característica de empregar dois tensores de flexibilidade danificada para levar em conta o comportamento "bimodular", em tração e em compressão do concreto. 
O procedimento sugerido por Cauvin e Testa para a obtenção daqueles tensores é baseado na aplicação do princípio da equivalência de deformação. Deve-se lembrar que somente a equivalência de energia garante a simetria maior do tensor constitutivo. Por essa razão, no modelo em questão a simetria maior do tensor de rigidez acaba por ser imposta. Com o artifício da imposição de simetria maior para E, mesmo valendo a equivalência de deformações, Cauvin e Testa conseguiram obter um tensor de dano que quando aplicado no tensor de rigidez simétrico íntegro leva a um tensor de rigidez danificado simétrico. Entretanto, é importante observar que esse modelo não se enquadra na proposta de Curnier, uma vez que não há um potencial de onde se deriva o tensor constitutivo.

Um primeiro tensor de dano é inspirado na danificação do concreto em tração uniaxial, onde microfissuras são distribuídas aleatoriamente em planos ortogonais à direção da solicitação que constituem planos de isotropia transversal (PTI - "Planar Transverse Isotropy" - Figura 2.4). A redação proposta do tensor de dano é a seguinte:

$\mathbf{D}_{\mathbf{T}}=\left(\mathrm{D}_{1}-\frac{v_{0} \mathrm{D}_{1}}{1-v_{0}}-2 \mathrm{D}_{4}\right) \mathbf{A} \otimes \mathbf{A}+\left(\frac{v_{0} \mathrm{D}_{1}}{1-v_{0}}\right) \mathbf{I} \otimes \mathbf{A}+\mathrm{D}_{4}(\underset{-}{\mathbf{A} \otimes} \underset{-}{\mathbf{I}}+\underset{-}{\mathbf{I}} \underset{-}{\mathbf{A}})$

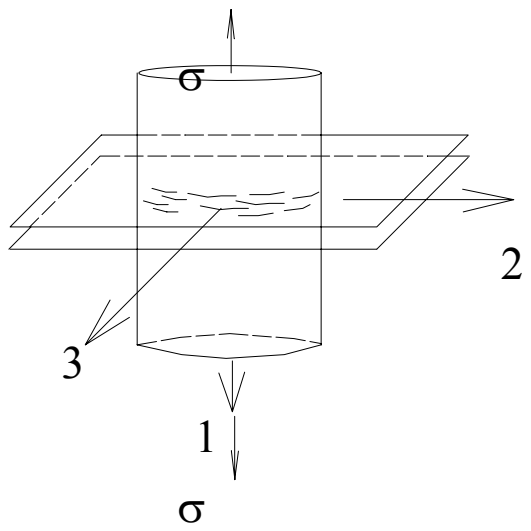

Figura 2.4 - Planos de isotropia transversal no caso de tração uniaxial no concreto.

Apenas dois módulos de rigidez são afetados pela danificação: $\mathrm{E}_{1}$ e $\mathrm{G}_{12}$. Cada um dos módulos é afetado por uma respectiva variável de dano, $\mathrm{D}_{1}$ e $\mathrm{D}_{4}$. A representação matricial do correspondente tensor de flexibilidade danificado é dada a seguir: 


$$
\mathbf{E}_{\mathbf{T}}^{-1}=\frac{1}{\mathrm{E}_{0}}\left[\begin{array}{cccccc}
\frac{\left(1-v_{0}\right)-2 v_{0}^{2} \mathrm{D}_{1}}{\left(1-v_{0}\right)\left(1-\mathrm{D}_{1}\right)} & -v_{0} & -v_{0} & 0 & 0 & 0 \\
-v_{0} & 1 & -v_{0} & 0 & 0 & 0 \\
-v_{0} & -v_{0} & 1 & 0 & 0 & 0 \\
0 & 0 & 0 & \left(1+v_{0}\right) & 0 & 0 \\
0 & 0 & 0 & 0 & \frac{\left(1+v_{0}\right)}{\left(1-D_{4}\right)} & 0 \\
0 & 0 & 0 & 0 & 0 & \frac{\left(1+v_{0}\right)}{\left(1-D_{4}\right)}
\end{array}\right]
$$

Seguindo a formulação proposta neste trabalho, o tensor constitutivo da equação (2.77) pode ser escrito como:

$$
\mathbf{C}_{\mathbf{T}}=\mathbf{C}_{\mathbf{0}}+\lambda^{*}{ }_{1}\left[\mathrm{D}_{1}, \mathrm{D}_{4}\right][\mathbf{A} \otimes \mathbf{A}]+\lambda_{4}\left[\mathrm{D}_{4}\right]\left[\mathbf{A} \otimes \bar{\otimes}_{-} \mathbf{I}+\mathbf{I}_{-} \otimes \mathbf{A}\right]
$$

onde $\lambda_{1}^{*}\left[D_{1}, D_{4}\right]=\frac{D_{1}\left(1-v_{0}-2 v_{0}^{2}\right)}{E_{0}\left(1-D_{1}\right)\left(1-v_{0}\right)}-2 \lambda_{4}\left[D_{4}\right]$ e $\lambda_{4}\left[D_{4}\right]=\frac{D_{4}\left(1+v_{0}\right)}{E_{0}\left(1-D_{4}\right)}$

Comparando-se a expressão (2.78) com uma forma geral para a flexibilidade danificada com isotropia transversal, obtém-se as seguintes relações para os módulos elásticos danificados em tração:

$$
\begin{aligned}
& E_{1}=\frac{E_{0}\left(1-D_{1}-v_{0}+v_{0} D_{1}\right)}{\left(1-v_{0}-2 v_{0}^{2} D_{1}\right)}, \quad v_{12}=v_{0} \frac{\left(1-D_{1}-v_{0}+v_{0} D_{1}\right)}{\left(1-v_{0}-2 v_{0}^{2} D_{1}\right)}=v_{0} \frac{E_{1}}{E_{0}} \\
& E_{2}=E_{0}, \quad v_{23}=v_{0}, \quad G_{23}=G_{0} \quad \text { e } \quad G_{12}=\frac{E_{0}\left(1-D_{4}\right)}{2\left(1+v_{0}\right)}
\end{aligned}
$$

O segundo tensor de dano é baseado na resposta esperada do concreto danificado em compressão uniaxial, onde microfissuras são distribuídas aleatoriamente no material mas definindo planos de isotropia na direção paralela à da solicitação ( CTI - “Cylindrical Transverse Isotropy” - Figura 2.5). A representação desse tensor é a seguinte: 


$$
\begin{aligned}
\mathbf{D}_{\mathbf{C}}= & \left(\mathrm{D}_{4}-2 \mathrm{D}_{3}\right)\left(\mathbf{A} \overline{-}_{-} \mathbf{I}+\underset{-}{\mathbf{I} \otimes \mathbf{A}}\right)+\left(\mathrm{D}_{3}-2 \mathrm{D}_{4}\right)(\mathbf{A} \otimes \mathbf{A})-\mathrm{D}_{3}(\mathbf{I} \otimes \mathbf{I}) \\
& +\mathrm{D}_{3}(\mathbf{A} \otimes \mathbf{I}+\mathbf{I} \otimes \mathbf{A})+2 \mathrm{D}_{3} \mathbf{I} \otimes \mathbf{I}
\end{aligned}
$$

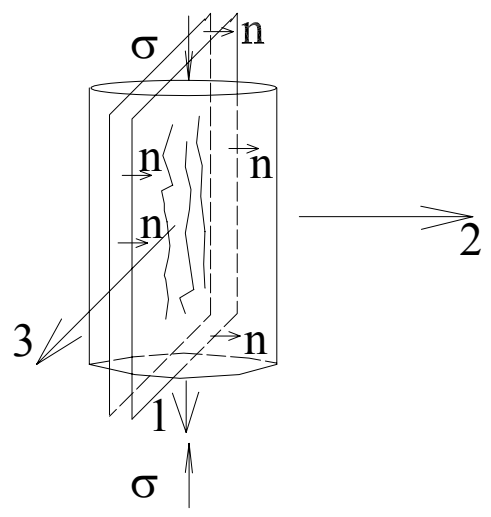

Figura 2.5 - Planos de isotropia transversal no caso de compressão uniaxial no concreto.

Apenas três módulos são afetados neste caso: $E_{2}, G_{23}$ e $G_{12}$. $O$ dano é então reduzido a dois parâmetros $\mathrm{D}_{3}$ e $\mathrm{D}_{4}$. A representação matricial do tensor de rigidez danificado é dada a seguir:

$\mathbf{C}_{\mathbf{C}}=\mathbf{E}_{\mathbf{C}}^{-1}=$

$\frac{1}{\mathrm{E}_{0}}\left[\begin{array}{cccccc}1 & -v_{0} & -v_{0} & 0 & 0 & 0 \\ -v_{0} & \frac{1-\left(1-v_{0}\right) \mathrm{D}_{3}}{1-2 \mathrm{D}_{3}} & -\frac{v_{0}+\left(1-v_{0}\right) \mathrm{D}_{3}}{1-2 \mathrm{D}_{3}} & 0 & 0 & 0 \\ -v_{0} & -\frac{v_{0}+\left(1-v_{0}\right) \mathrm{D}_{3}}{1-2 \mathrm{D}_{3}} & \frac{1-\left(1-v_{0}\right) \mathrm{D}_{3}}{1-2 \mathrm{D}_{3}} & 0 & 0 & 0 \\ 0 & 0 & 0 & \frac{\left(1+v_{0}\right)}{1-2 \mathrm{D}_{3}} & 0 & 0 \\ 0 & 0 & 0 & 0 & \frac{\left(1+v_{0}\right)}{\left(1-\mathrm{D}_{4}\right)} & 0 \\ 0 & 0 & 0 & 0 & 0 & \frac{\left(1+v_{0}\right)}{\left(1-\mathrm{D}_{4}\right)}\end{array}\right]$

O tensor constitutivo acima pode ainda ser escrito na forma:

$$
\begin{aligned}
\mathbf{C}_{\mathbf{C}}= & \mathbf{C}_{\mathbf{0}}+\lambda_{3}\left[\mathrm{D}_{3}\right][\mathbf{A} \otimes \mathbf{I}+\mathbf{I} \otimes \mathbf{A}]-\lambda_{3}\left[\mathrm{D}_{3}\right][\mathbf{I} \otimes \mathbf{I}]+\lambda_{3}{ }_{3}\left[\mathrm{D}_{3}, \mathrm{D}_{4}\right]\left[\underset{-}{\mathbf{A} \otimes \mathbf{I}}+\mathbf{I} \otimes{ }_{-} \mathbf{A}\right] \\
& +2 \lambda_{3}\left[\mathrm{D}_{3}\right][\mathbf{I} \otimes \mathbf{I}]+\left(3 \lambda_{3}\left[\mathrm{D}_{3}\right]+2 \lambda^{*}{ }_{3}\left[\mathrm{D}_{3}, \mathrm{D}_{4}\right]\right)[\mathbf{A} \otimes \mathbf{A}]
\end{aligned}
$$


onde $\lambda_{3}\left[D_{3}\right]=\frac{D_{3}\left(1+v_{0}\right)}{E_{0}\left(1-2 D_{3}\right)}$ e $\lambda_{3}^{*}\left[D_{3}, D_{4}\right]=\frac{D_{4}\left(1+v_{0}\right)}{E_{0}\left(1-D_{4}\right)}-2 \lambda_{3}\left[D_{3}\right]$.

Comparando-se a expressão anterior com a de uma flexibilidade danificada com isotropia transversal, obtém-se como resultado os valores dos módulos elásticos danificados em compressão.

$$
\begin{array}{ll}
\mathrm{E}_{1}=\mathrm{E}_{0}, \quad v_{12}=v_{0} & \\
\mathrm{E}_{2}=\frac{\mathrm{E}_{0}\left(1-2 \mathrm{D}_{3}\right)}{\left(1-\mathrm{D}_{3}+v_{0} \mathrm{D}_{3}\right)}, & v_{23}=\frac{\left(v_{0}+\mathrm{D}_{3}-v_{0} \mathrm{D}_{3}\right)}{\left(1-\mathrm{D}_{3}+v_{0} \mathrm{D}_{3}\right)}=\frac{\left(v_{0}+\mathrm{D}_{3}-v_{0} \mathrm{D}_{3}\right)}{\left(1-2 \mathrm{D}_{3}\right)} \frac{\mathrm{E}_{2}}{\mathrm{E}_{0}} \\
\mathrm{G}_{23}=\frac{\mathrm{E}_{0}\left(1-2 \mathrm{D}_{3}\right)}{2\left(1+v_{0}\right)} & \text { e } \quad \mathrm{G}_{12}=\frac{\mathrm{E}_{0}\left(1-\mathrm{D}_{4}\right)}{2\left(1+v_{0}\right)}
\end{array}
$$

Alguns aspectos deste modelo merecem comentários. Em primeiro lugar, o tensor de dano em tração não possui simetria. Isso é devido à imposição artificiosa de restrições para garantir um tensor constitutivo danificado simétrico apesar da equivalência de deformação. Tal imposição é arbitrária e tem como estratégia deduzir do tensor constitutivo simétrico a parcela correspondente ao tensor de dano, o que acarreta na não-simetria do mesmo. Cauvin e Testa também não propõem qualquer critério para a aplicação de um ou outro tensor de dano.

Outro detalhe importante é que imaginando-se que seja válido o critério de Curnier para a identificação de estados predominantes de tração e de compressão, os tensores de Cauvin não obedeceriam à condição de salto tangencial nulo no tensor constitutivo relativa à resposta "bimodular". Observa-se que os valores de $\mathrm{G}_{12}=\mathrm{G}_{13}$ e $\mathrm{G}_{23}$ na tração deveriam possuir os mesmos valores em compressão para satisfazer a condição de salto tangencial nulo do tensor constitutivo na proposta de Curnier, o que não acontece com o modelo.

Por último, o módulo de elasticidade em compressão $\mathrm{E}_{1}=\mathrm{E}_{0}$ e o coeficiente de Poisson $v_{12}=v_{0}(2.83)$ não são danificados, o que não condiz com a realidade como seria de se esperar, por exemplo, num caso de compressão uniaxial na direção 1. Pode-se pensar que de um ponto de vista teórico a não danificação na direção da solicitação poderia ser justificável, pois é sabido que as fissuras tendem a se posicionar paralelamente ao carregamento. Porém existem fissuras que possuem 
pequena inclinação e que levam à redução de rigidez do concreto em compressão na direção do carregamento.

No capítulo seguinte propõe-se um modelo de dano procurando-se contornar alguns problemas evidenciados nos modelos aqui apresentados. 


\section{PROPOSTA DE MODELO PARA O CONCRETO}

\subsection{INTRODUÇÃO}

Este capítulo apresenta a formulação de um modelo de dano para o concreto, incorporando-se algumas das características do seu comportamento mecânico, vistas no capítulo 1 deste trabalho, e tendo como base o formalismo matemático proposto no capítulo anterior. Além disso, procura-se atender ao princípio de equivalência de energia entre meio real danificado e meio contínuo equivalente estabelecido na Mecânica do Dano [LEMAITRE (1996)].

$\mathrm{O}$ concreto é aqui entendido como um material que pertence à categoria dos meios inicialmente isótropos que passam a apresentar isotropia transversal e resposta bimodular induzidas pelo dano.

No que segue, descrevem-se os tensores de dano correspondentes aos estados predominantes de tração e de compressão. Em seguida, o formalismo geral do modelo é exposto partindo-se do potencial termodinâmico e de suas relações derivadas. Propõem-se ainda critérios para a divisão do espaço das deformações, para a ativação e evolução da danificação e para a identificação do plano local de isotropia transversal do material. Finalmente, apresentam-se expressões para as leis de evolução das variáveis de dano para o modelo proposto. 


\subsection{TENSOR CONSTITUTIVO CONSISTENTE COM O PRINCÍPIO DE EQUIVALÊNCIA DE ENERGIA}

O conceito de equivalência de energia, proposto por CORDEBOIS \& SIDOROFF (1979), postula que a energia elástica de um material danificado possa ser reproduzida de forma equivalente num meio contínuo. Atendendo a esse princípio, os tensores constitutivos de rigidez e de flexibilidade do meio contínuo equivalente resultam simétricos e dados pelas seguintes relações:

$\mathbf{E}=(\mathbf{I I}-\mathbf{D}) \mathbf{E}_{0}(\mathbf{I I}-\mathbf{D})^{\mathbf{T}} \quad$ ou $\quad \mathbf{C}=(\mathbf{I I}-\mathbf{D})^{-\mathbf{T}} \mathbf{C}_{0}(\mathbf{I I}-\mathbf{D})^{-1}$

onde o tensor de quarta ordem (II - D) reúne em suas componentes as variáveis que quantificam o dano.

Tendo-se em vista o caráter bimodular gerado pela danificação, é interessante definir dois tensores de dano, um para estados predominantes de tração e um outro para estados predominantes de compressão. O critério para definir estados predominantes de tração e de compressão será discutido mais adiante.

Observa-se que formas gerais que permitem contemplar a anisotropia induzida pela danificação podem ser consideradas na definição do tensor de dano de quarta ordem. Neste trabalho para a definição de $\mathbf{D}$, opta-se por uma forma dita de dano escalar dada por: $\mathbf{D}=\mathrm{f}_{\mathrm{j}}\left(\mathrm{D}_{\mathrm{i}}\right) \mathbf{M}_{\mathbf{j}}$, onde $\mathrm{f}_{\mathrm{j}}\left(\mathrm{D}_{\mathrm{i}}\right)$ são funções de valor escalar das variáveis escalares de dano escolhidas e $\mathbf{M}_{\mathbf{j}}$ são tensores de anisotropia. No caso deste modelo, adotam-se para $\mathbf{M}_{\mathbf{j}}$ tensores que permitem representar a isotropia transversal.

Para estados predominantes de tração, propõe-se o seguinte tensor de dano escalar:

$\mathbf{D}_{\mathbf{T}}=\mathrm{f}_{1}\left(\mathrm{D}_{1}, \mathrm{D}_{4}, \mathrm{D}_{5}\right)(\mathbf{A} \otimes \mathbf{A})+2 \mathrm{f}_{2}\left(\mathrm{D}_{4}, \mathrm{D}_{5}\right)\left[\left(\mathbf{A} \otimes \vec{\otimes} \mathbf{I}+\mathbf{I}_{-} \otimes \mathbf{A}\right)-(\mathbf{A} \otimes \mathbf{A})\right]$

sendo $f_{1}\left(D_{1}, D_{4}, D_{5}\right)=D_{1}-2 f_{2}\left(D_{4}, D_{5}\right)$ e $f_{2}\left(D_{4}, D_{5}\right)=1-\left(1-D_{4}\right)\left(1-D_{5}\right)$. 
O tensor de dano apresenta duas variáveis escalares na sua composição $\left(D_{1}\right.$ e $\mathrm{D}_{4}$ ) e uma terceira variável de dano $\mathrm{D}_{5}$, ativada somente se tiver havido compressão prévia com danificação correspondente. A variável $\mathrm{D}_{1}$ representa a danificação na direção perpendicular ao plano local de isotropia transversal do material e $\mathrm{D}_{4}$ é a variável representativa da danificação gerada pelo cisalhamento entre as bordas das fissuras pertencentes àquele plano.

Para uma visualização do tensor de dano em tração, ilustra-se o caso de tração uniaxial da figura 2.4 do capítulo anterior, onde o plano de isotropia transversal é coincidente com o plano 2-3. Sendo assim, o tensor de dano em tração assume a seguinte representação matricial:

$$
\mathbf{D}_{\mathbf{T}}=\left[\begin{array}{cccccc}
\mathrm{D}_{1} & 0 & 0 & 0 & 0 & 0 \\
0 & 0 & 0 & 0 & 0 & 0 \\
0 & 0 & 0 & 0 & 0 & 0 \\
0 & 0 & 0 & 0 & 0 & 0 \\
0 & 0 & 0 & 0 & 1-\left(1-\mathrm{D}_{4}\right)\left(1-\mathrm{D}_{5}\right) & 0 \\
0 & 0 & 0 & 0 & 0 & 1-\left(1-\mathrm{D}_{4}\right)\left(1-\mathrm{D}_{5}\right)
\end{array}\right]
$$

Na eq. (3.2), o tensor I é o tensor identidade de segunda ordem e o tensor A é, por definição, [CURNIER, HE \& ZYSSET (1995)], formado pelo produto tensorial do versor perpendicular ao plano de isotropia transversal por ele mesmo. As operações produtos tensoriais entre os tensores de segunda ordem I e A que aparecem na eq. (3.2) e que serão utilizadas durante toda a formulação estão descritas no apêndice deste trabalho.

Para estados predominantes de compressão, propõe-se para o tensor de dano a seguinte relação:

$$
\begin{aligned}
\mathbf{D}_{\mathbf{C}}= & \mathrm{f}_{1}\left(\mathrm{D}_{2}, \mathrm{D}_{4}, \mathrm{D}_{5}\right)(\mathbf{A} \otimes \mathbf{A})+\mathrm{f}_{2}\left(\mathrm{D}_{3}\right)[(\mathbf{I} \otimes \mathbf{I})-(\mathbf{A} \otimes \mathbf{A})] \\
& +2 \mathrm{f}_{3}\left(\mathrm{D}_{4}, \mathrm{D}_{5}\right)[(\mathbf{A} \otimes \mathbf{I} \mathbf{I}+\mathbf{I} \otimes \mathbf{A})-(\mathbf{A} \otimes \mathbf{A})]
\end{aligned}
$$

sendo $f_{1}\left(D_{2}, D_{4}, D_{5}\right)=D_{2}-2 f_{3}\left(D_{4}, D_{5}\right), f_{2}\left(D_{3}\right)=D_{3}$ e $f_{3}\left(D_{4}, D_{5}\right)=1-\left(1-D_{4}\right)\left(1-D_{5}\right)$. 
Notam-se três variáveis escalares na sua composição: $\mathrm{D}_{2}, \mathrm{D}_{3}$ e $\mathrm{D}_{5}$, além de $\mathrm{D}_{4}$, relacionada a efeitos de tração pré-existentes. A variável $\mathrm{D}_{2}$ (danificação perpendicular ao plano local de isotropia transversal do material) penaliza como se verá mais adiante, o módulo de elasticidade nessa direção e juntamente com $\mathrm{D}_{3}$ (representante da danificação no plano de isotropia transversal) penaliza o coeficiente de Poisson em planos perpendiculares ao de isotropia transversal. Observa-se que, com a forma descrita, é possível capturar a danificação dos módulos de cisalhamento e ao mesmo tempo atender à hipótese de salto tangencial nulo do tensor constitutivo [ver capítulo 2 eq. (2.27)].

Para uma visualização do tensor de dano em compressão, ilustra-se o caso de compressão uniaxial da figura 2.5 do capítulo anterior, onde o plano de isotropia transversal é coincidente com o plano 2-3. Sendo assim, o tensor de dano em compressão assume a seguinte representação matricial:

$$
\mathbf{D}_{\mathbf{C}}=\left[\begin{array}{cccccc}
\mathrm{D}_{2} & 0 & 0 & 0 & 0 & 0 \\
0 & \mathrm{D}_{3} & 0 & 0 & 0 & 0 \\
0 & 0 & \mathrm{D}_{3} & 0 & 0 & 0 \\
0 & 0 & 0 & 0 & 0 & 0 \\
0 & 0 & 0 & 0 & 1-\left(1-\mathrm{D}_{4}\right)\left(1-\mathrm{D}_{5}\right) & 0 \\
0 & 0 & 0 & 0 & 0 & 1-\left(1-\mathrm{D}_{4}\right)\left(1-\mathrm{D}_{5}\right)
\end{array}\right]
$$

É possível mostrar que as formas adotadas para $\mathbf{D}_{\mathbf{T}}$ e $\mathbf{D}_{\mathbf{C}}$ atendem à (3.1) no sentido de que proporcionam um tensor de rigidez simétrico. Finalmente, os tensores constitutivos resultantes são descritos por:

$$
\begin{aligned}
& \mathbf{E}_{\mathbf{T}}=\lambda_{11}[\mathbf{I} \otimes \mathbf{I}]+2 \mu_{1}[\mathbf{I} \otimes \mathbf{I}]-\lambda_{22}^{+}\left(\mathrm{D}_{1}, \mathrm{D}_{4}, \mathrm{D}_{5}\right)[\mathbf{A} \otimes \mathbf{A}] \\
& -\lambda_{12}^{+}\left(\mathrm{D}_{1}\right)[\mathbf{A} \otimes \mathbf{I}+\mathbf{I} \otimes \mathbf{A}]-\mu_{2}\left(\mathrm{D}_{4}, \mathrm{D}_{5}\right)\left[\mathbf{A} \bar{\otimes}_{-} \mathbf{I}+\mathbf{I} \otimes \mathbf{A}\right] \\
& \mathbf{E}_{\mathbf{C}}=\lambda_{11}[\mathbf{I} \otimes \mathbf{I}]+2 \mu_{1}[\mathbf{I} \otimes \bar{I}]-\lambda_{22}^{-}\left(\mathrm{D}_{2}, \mathrm{D}_{3}, \mathrm{D}_{4}, \mathrm{D}_{5}\right)[\mathbf{A} \otimes \mathbf{A}] \\
& -\lambda_{12}^{-}\left(\mathbf{D}_{2}, \mathbf{D}_{3}\right)[\mathbf{A} \otimes \mathbf{I}+\mathbf{I} \otimes \mathbf{A}]-\lambda_{11}^{-}\left(\mathbf{D}_{3}\right)[\mathbf{I} \otimes \mathbf{I}] \\
& -\frac{\left(1-2 v_{0}\right)}{v_{0}} \lambda_{11}^{-}\left(\mathbf{D}_{3}\right)[\mathbf{I} \bar{\otimes} \mathbf{I}]-\mu_{2}\left(\mathrm{D}_{4}, \mathrm{D}_{5}\right)[\underset{-}{\bar{\otimes}} \mathbf{I}+\mathbf{I} \underset{-}{\bar{\otimes}} \mathbf{A}]
\end{aligned}
$$


onde:

$\lambda_{11}=\lambda_{0} ; \mu_{1}=\mu_{0}$

os outros parâmetros só existem para dano não-nulo, evidenciando dessa forma a anisotropia e bimodularidade induzidas pelo dano, e são definidos por:

$$
\begin{aligned}
& \lambda_{22}^{+}\left(D_{1}, D_{4}, D_{5}\right)=\left(\lambda_{0}+2 \mu_{0}\right)\left(2 D_{1}-D_{1}^{2}\right)-2 \lambda_{12}^{+}\left(D_{1}\right)-2 \mu_{2}\left(D_{4}, D_{5}\right) \\
& \lambda_{12}^{+}\left(D_{1}\right)=\lambda_{0} D_{1} ; \quad \mu_{2}\left(D_{4}, D_{5}\right)=2 \mu_{0}\left[1-\left(1-D_{4}\right)^{2}\left(1-D_{5}\right)^{2}\right] \\
& \lambda_{22}^{-}\left(D_{2}, D_{3}, D_{4}, D_{5}\right)=\left(\lambda_{0}+2 \mu_{0}\right)\left(2 D_{2}-D_{2}^{2}\right)-2 \lambda_{12}^{-}\left(D_{2}, D_{3}\right) \\
& +\frac{\left(v_{0}-1\right)}{v_{0}} \lambda_{11}^{-}\left(D_{3}\right)-2 \mu_{2}\left(D_{4}, D_{5}\right) \\
& \lambda_{12}^{-}\left(D_{2}, D_{3}\right)=\lambda_{0}\left[\left(1-D_{3}\right)^{2}-\left(1-D_{2}\right)\left(1-D_{3}\right)\right] \\
& \lambda_{11}^{-}\left(\mathrm{D}_{3}\right)=\lambda_{0}\left(2 \mathrm{D}_{3}-\mathrm{D}_{3}^{2}\right)
\end{aligned}
$$

Numa interpretação puramente matricial, os diferentes produtos tensoriais das eqs. (3.6) e (3.7) possuem a função de alocar as constantes que os multiplicam em determinadas posições nos tensores de rigidez.

Para uma melhor visualização dos tensores constitutivos é interessante representá-los segundo uma forma matricial. Com esse objetivo adota-se, por conveniência, um sistema de coordenadas locais tal que a direção 1 seja perpendicular ao plano de isotropia transversal. Assim sendo, as representações matriciais dos tensores (3.6) e (3.7) resultam:

$$
\mathrm{E}_{\mathrm{T}}=\left[\begin{array}{cccccc}
\left(\lambda_{0}+2 \mu_{0}\right)\left(1-\mathrm{D}_{1}\right)^{2} & \lambda_{0}\left(1-\mathrm{D}_{1}\right) & \lambda_{0}\left(1-\mathrm{D}_{1}\right) & 0 & 0 & 0 \\
\lambda_{0}\left(1-\mathrm{D}_{1}\right) & \left(\lambda_{0}+2 \mu_{0}\right) & \lambda_{0} & 0 & 0 & 0 \\
\lambda_{0}\left(1-\mathrm{D}_{1}\right) & \lambda_{0} & \left(\lambda_{0}+2 \mu_{0}\right) & 0 & 0 & 0 \\
0 & 0 & 0 & \mu_{0} & 0 & 0 \\
0 & 0 & 0 & 0 & \mu_{0}\left(1-\mathrm{D}_{4}\right)^{2}\left(1-\mathrm{D}_{5}\right)^{2} & 0 \\
0 & 0 & 0 & 0 & 0 & \mu_{0}\left(1-\mathrm{D}_{4}\right)^{2}\left(1-\mathrm{D}_{5}\right)^{2}
\end{array}\right]
$$


$\mathrm{E}_{\mathrm{C}}=\left[\begin{array}{cccccc}\left(\lambda_{0}+2 \mu_{0}\right)\left(1-\mathrm{D}_{2}\right)^{2} & \lambda_{0}\left(1-\mathrm{D}_{2}\right)\left(1-\mathrm{D}_{3}\right) & \lambda_{0}\left(1-\mathrm{D}_{2}\right)\left(1-\mathrm{D}_{3}\right) & 0 & 0 & 0 \\ \lambda_{0}\left(1-\mathrm{D}_{2}\right)\left(1-\mathrm{D}_{3}\right) & \left(\lambda_{0}+2 \mu_{0}\right)\left(1-\mathrm{D}_{3}\right)^{2} & \lambda_{0}\left(1-\mathrm{D}_{3}\right)^{2} & 0 & 0 & 0 \\ \lambda_{0}\left(1-\mathrm{D}_{2}\right)\left(1-\mathrm{D}_{3}\right) & \lambda_{0}\left(1-\mathrm{D}_{3}\right)^{2} & \left(\left(\lambda_{0}+2 \mu_{0}\right)\left(1-\mathrm{D}_{3}\right)^{2}\right. & 0 & 0 & 0 \\ 0 & 0 & 0 & \mu_{0} & 0 & 0 \\ 0 & 0 & 0 & 0 & \mu_{0}\left(1-\mathrm{D}_{4}\right)^{2}\left(1-\mathrm{D}_{5}\right)^{2} & 0 \\ 0 & 0 & 0 & 0 & 0 & \mu_{0}\left(1-\mathrm{D}_{4}\right)^{2}\left(1-\mathrm{D}_{5}\right)^{2}\end{array}\right]$

\subsection{FORMA GERAL DO MODELO}

Seguindo o formalismo do item 2.3 do capítulo anterior, o modelo proposto exprime-se pela forma invariante explicitada no que segue.

$$
\begin{gathered}
\mathrm{W}(\varepsilon)=\rho \psi(\varepsilon):=\left\{\begin{array}{l}
\mathrm{W}_{-}(\varepsilon) \text { se } \mathrm{g}\left(\varepsilon, \mathbf{D}_{\mathrm{T}}, \mathbf{D}_{\mathbf{C}}\right)<0, \\
\mathrm{~W}_{+}(\varepsilon) \text { se } \mathrm{g}\left(\varepsilon, \mathbf{D}_{\mathrm{T}}, \mathbf{D}_{\mathbf{C}}\right)>0,
\end{array} \text { e } \mathrm{W}_{-}=\mathrm{W}_{+}=\mathrm{W}_{0} \text { se } \mathbf{D}_{\mathbf{T}}=\mathbf{D}_{\mathbf{C}}=\mathbf{0}(\mathbf{3 . 1 2})\right. \\
\mathrm{W}_{+}=\rho \psi_{+}(\varepsilon)=\frac{\lambda_{11}}{2} \operatorname{tr}^{2}(\varepsilon)+\mu_{1} \operatorname{tr}\left(\varepsilon^{2}\right)-\frac{\lambda_{22}^{+}\left(\mathrm{D}_{1}, \mathrm{D}_{4}, \mathrm{D}_{5}\right)}{2} \operatorname{tr}^{2}(\mathbf{A} \varepsilon)-\lambda_{12}^{+}\left(\mathrm{D}_{1}\right) \operatorname{tr}(\varepsilon) \operatorname{tr}(\mathbf{A} \varepsilon) \\
\quad-\mu_{2}\left(\mathrm{D}_{4}, \mathrm{D}_{5}\right) \operatorname{tr}\left(\mathbf{A} \varepsilon^{2}\right) \\
\mathrm{W}_{-}=\rho \psi_{-}(\varepsilon)=\frac{\lambda_{11}}{2} \operatorname{tr}^{2}(\varepsilon)+\mu_{1} \operatorname{tr}\left(\varepsilon^{2}\right)-\frac{\lambda_{22}^{-}\left(\mathrm{D}_{2}, \mathrm{D}_{3}, \mathrm{D}_{4}, \mathrm{D}_{5}\right)}{2} \operatorname{tr}^{2}(\mathbf{A} \varepsilon)-\lambda_{12}^{-}\left(\mathrm{D}_{2}, \mathrm{D}_{3}\right) \operatorname{tr}(\varepsilon) \operatorname{tr}(\mathbf{A} \varepsilon) \\
-\frac{\lambda_{11}^{-}\left(\mathrm{D}_{3}\right)}{2} \operatorname{tr}^{2}(\varepsilon)-\frac{\left(1-2 v_{0}\right)}{2 v_{0}} \lambda_{11}^{-}\left(\mathrm{D}_{3}\right) \operatorname{tr}[(\mathbf{I} \otimes \mathbf{\otimes} \mathbf{I}) \varepsilon]^{2}-\mu_{2}\left(\mathrm{D}_{4}, \mathrm{D}_{5}\right) \operatorname{tr}\left(\mathbf{A} \varepsilon^{2}\right)
\end{gathered}
$$

onde os coeficientes $\lambda$ e $\mu$ são os mesmos descritos anteriormente [ eq. (3.8) e (3.9)] e $\mathrm{W}_{0}$ é o potencial elástico do material íntegro.

O tensor de tensões obtém-se do gradiente do potencial elástico resultando:

$$
\sigma(\varepsilon)=\nabla_{\varepsilon} \rho \psi(\varepsilon)=\left\{\begin{array}{lll}
\sigma_{-}(\varepsilon)=\nabla_{\varepsilon} \rho \psi_{-}(\varepsilon) & \text { se } & \mathrm{g}\left(\varepsilon, \mathbf{D}_{\mathrm{T}}, \mathbf{D}_{\mathbf{C}}\right)<0 \\
\sigma_{+}(\varepsilon)=\nabla_{\varepsilon} \rho \psi_{+}(\varepsilon) & \text { se } & \mathrm{g}\left(\varepsilon, \mathbf{D}_{\mathrm{T}}, \mathbf{D}_{\mathbf{C}}\right)>0
\end{array}\right.
$$




$$
\begin{aligned}
\sigma_{+}(\varepsilon) & =\rho \frac{\partial \psi_{+}}{\partial \varepsilon}=\lambda_{11} \operatorname{tr}(\varepsilon) \mathbf{I}+2 \mu_{1} \varepsilon-\lambda_{22}^{+}\left(\mathrm{D}_{1}, \mathrm{D}_{4}, \mathrm{D}_{5}\right) \operatorname{tr}(\mathbf{A} \varepsilon) \mathbf{A} \\
& -\lambda_{12}^{+}\left(\mathrm{D}_{1}\right)(\operatorname{tr}(\varepsilon) \mathbf{A}+\operatorname{tr}(\mathbf{A} \varepsilon) \mathbf{I})-\mu_{2}\left(\mathrm{D}_{4}, \mathrm{D}_{5}\right)(\mathbf{A} \varepsilon+\varepsilon \mathbf{A}) \\
\sigma_{-}(\varepsilon) & =\rho \frac{\partial \psi_{-}}{\partial \varepsilon}=\lambda_{11} \operatorname{tr}(\varepsilon) \mathbf{I}+2 \mu_{1} \varepsilon-\lambda_{22}^{-}\left(\mathrm{D}_{2}, \mathrm{D}_{3}, \mathrm{D}_{4}, \mathrm{D}_{5}\right) \operatorname{tr}(\mathrm{A} \varepsilon) \mathbf{A} \\
& -\lambda_{12}^{-}\left(\mathrm{D}_{2}, \mathrm{D}_{3}\right)(\operatorname{tr}(\varepsilon) \mathbf{A}+\operatorname{tr}(\mathbf{A} \varepsilon) \mathbf{I})-\lambda_{11}^{-}\left(\mathrm{D}_{3}\right) \operatorname{tr}(\varepsilon) \mathbf{I}-\frac{\left(1-2 v_{0}\right)}{v_{0}} \lambda_{11}^{-}\left(\mathrm{D}_{3}\right)(\mathbf{I} \otimes \mathbf{I} \mathbf{I}) \varepsilon \\
& -\mu_{2}\left(\mathrm{D}_{4}, \mathrm{D}_{5}\right)(\mathbf{A} \boldsymbol{\varepsilon}+\varepsilon \mathbf{A})
\end{aligned}
$$

O tensor constitutivo de rigidez também é obtido do potencial elástico:

$\mathbf{E}(\varepsilon):=\left\{\begin{array}{lll}\mathbf{E}_{-}(\varepsilon)=\nabla_{\varepsilon}^{2} \rho \psi_{-}(\varepsilon) & \text { se } & \mathrm{g}\left(\varepsilon, \mathbf{D}_{\mathrm{T}}, \mathbf{D}_{\mathrm{C}}\right)<0, \\ \mathbf{E}_{+}(\varepsilon)=\nabla_{\varepsilon}^{2} \rho \psi_{+}(\varepsilon) & \text { se } & \mathrm{g}\left(\varepsilon, \mathbf{D}_{\mathrm{T}}, \mathbf{D}_{\mathbf{C}}\right)>0,\end{array}\right.$

$\mathbf{E}_{+}(\varepsilon)=\rho \frac{\partial^{2} \Psi_{+}}{\partial \varepsilon^{2}}=\mathbf{E}_{\mathbf{T}}$

$\mathbf{E}_{-}(\varepsilon)=\rho \frac{\partial^{2} \Psi_{-}}{\partial \varepsilon^{2}}=\mathbf{E}_{\mathbf{C}}$

onde o tensor constitutivo $\mathbf{E}_{\mathbf{T}}$ é dado pela expressão (3.6) e $\mathbf{E}_{\mathbf{C}}$ pela (3.7).

As variáveis associadas às variáveis de dano em tração $\left(\mathrm{D}_{1}, \mathrm{D}_{4}\right)$ e em compressão $\left(\mathrm{D}_{2}, \mathrm{D}_{3}, \mathrm{D}_{5}\right)$ podem ser interpretadas como taxas de energia liberada durante o processo de evolução de dano, e também são derivadas do potencial W:

$$
\begin{array}{ll}
Y_{1}\left(D_{1}\right)=-\rho \frac{\partial \psi_{+}}{\partial D_{1}} & Y_{4}\left(D_{4}\right)=-\rho \frac{\partial \psi_{+}}{\partial D_{4}} \\
Y_{2}\left(D_{1}\right)=-\rho \frac{\partial \psi_{-}}{\partial D_{2}} & Y_{3}\left(D_{3}\right)=-\rho \frac{\partial \psi_{-}}{\partial D_{3}} \quad Y_{5}\left(D_{5}\right)=-\rho \frac{\partial \psi_{-}}{\partial D_{5}}
\end{array}
$$

As variáveis associadas ao dano podem ser empregadas na definição das funções critério de danificação. Tais funções e o critério para a divisão do espaço das deformações $\left[g\left(\varepsilon, \mathbf{D}_{\mathbf{T}}, \mathbf{D}_{\mathbf{C}}\right)\right]$ serão objeto de análise nos próximos itens. 


\subsection{CRITÉRIO PARA A DIVISÃO DO ESPAÇO DAS DEFORMAÇÕES}

O modelo proposto inclui dois tensores de dano que permitem levar em conta o aspecto bimodular induzido pela danificação no comportamento do concreto. Com isso, surge a necessidade de um critério para caracterizar estados predominantes de tração e de compressão para indicar qual tensor de dano deverá ser utilizado.

Em CURNIER, HE \& ZYSSET (1995) define-se uma hipersuperfície, no espaço das tensões ou das deformações, a ser empregada como critério para a identificação das respostas constitutivas de compressão ou de tração. Neste trabalho adota-se uma forma particular para a hipersuperfície no espaço das deformações: um hiperplano g $(\varepsilon)$, caracterizado por sua normal unitária $\mathbf{N}(\|\mathbf{N}\|=1)$.

Este critério é ainda estendido de modo que o estado de dano existente passe a ter influência sobre a definição do hiperplano. Portanto, para a função g propõe-se a seguinte representação:

$\mathrm{g}\left(\varepsilon, \mathbf{D}_{\mathbf{T}}, \mathbf{D}_{\mathbf{C}}\right)=\mathbf{N}\left(\mathbf{D}_{\mathbf{T}}, \mathbf{D}_{\mathbf{C}}\right) \cdot \varepsilon^{\mathrm{e}}$

Discute-se em seguida, com mais detalhes, a divisão do espaço das deformações resultante neste modelo. Nesse sentido é interessante reproduzir as relações (2.33) e (2.34) do capítulo anterior, que se referem à divisão do espaço por um hiperplano numa situação sem danificação:

$g(\varepsilon)=\gamma_{\mathrm{a}} \operatorname{tr} \mathbf{A}_{\mathbf{a}} \varepsilon^{\mathbf{e}}=\operatorname{tr}\left[\left(\gamma_{\mathrm{a}} \mathbf{A}_{\mathbf{a}}\right) \varepsilon^{\mathbf{e}}\right]=\mathbf{N} \cdot \varepsilon^{\mathbf{e}}=0, \quad(\mathrm{a}=1, \mathrm{~d})$,

$\mathbf{N}=\nabla_{\varepsilon} g=\frac{\partial g}{\partial \mathbf{I}_{\mathrm{a}}} \nabla_{\varepsilon} \mathrm{I}_{\mathrm{a}}=\gamma_{\mathrm{a}} \mathbf{A}_{\mathrm{a}}$, onde os coeficientes $\gamma_{\mathrm{a}}$ são tais que: $\|\mathbf{N}\|=1$

Para o caso de isotropia tem-se que o sub-índice "a" deve ser igual a 1 e o tensor $\mathbf{A}_{\mathbf{1}}=\mathbf{I}$. Nessa condição e ainda na ausência de dano, a deformação elástica volumétrica nula define a interface entre os dois subdomínios de deformações. De fato, 


$$
\begin{aligned}
& \mathbf{N}=\gamma_{1} \mathbf{A}_{1}=\gamma_{1} \mathbf{I}, \quad\left(\|\mathbf{N}\|=1 \Leftrightarrow \gamma_{1}=\frac{1}{\sqrt{3}}\right) . \\
& g(\varepsilon)=\mathbf{N} \cdot \varepsilon^{\mathbf{e}}=0, g(\varepsilon)=\frac{1}{\sqrt{3}} \mathbf{I} \cdot \varepsilon^{\mathbf{e}}=\frac{1}{\sqrt{3}} \operatorname{tr}\left(\varepsilon^{\mathbf{e}}\right)=\frac{1}{\sqrt{3}} \varepsilon_{\mathrm{V}}^{\mathrm{e}}=0 \text { donde } \varepsilon_{\mathrm{V}}^{\mathrm{e}}=0
\end{aligned}
$$

sendo $\varepsilon_{\mathrm{V}}^{\mathrm{e}}$ a deformação elástica volumétrica.

Por outro lado, para o caso de isotropia transversal, sem danificação, na (3.23a), os valores assumidos para o sub-índice e os tensores $\mathbf{A}_{\mathbf{a}}$ são: a $=1,2, \mathbf{A}_{\mathbf{1}}=\mathbf{I}$ e $\mathbf{A}_{2}=\mathbf{A}$. Segue daí que:

$\mathbf{N}=\gamma_{1} \mathbf{A}_{1}+\gamma_{2} \mathbf{A}_{2}=\gamma_{1} \cdot \mathbf{I}+\gamma_{2} \cdot \mathbf{A}$

$g(\varepsilon)=\mathbf{N} \cdot \varepsilon^{\mathbf{e}}=0, \quad g(\varepsilon)=\left(\gamma_{1} \mathbf{I}+\gamma_{2} \mathbf{A}\right) \cdot \varepsilon^{\mathbf{e}}=\gamma_{1}\left(\mathbf{I} \cdot \varepsilon^{\mathbf{e}}\right)+\gamma_{2} \mathbf{A} \cdot \varepsilon^{\mathbf{e}}=\gamma_{1} \operatorname{tr}\left(\varepsilon^{\mathbf{e}}\right)+\gamma_{2} \operatorname{tr}(\mathbf{A}$. $\left.\varepsilon^{\mathbf{e}}\right)=0$

$\mathrm{g}(\varepsilon)=\gamma_{1} \varepsilon_{\mathrm{V}}^{\mathrm{e}}+\gamma_{2}\left(\varepsilon_{11}^{\mathrm{e}} \mathrm{a}_{1}^{2}+\varepsilon_{22}^{\mathrm{e}} \mathrm{a}_{2}^{2}+\varepsilon_{33}^{\mathrm{e}} \mathrm{a}_{3}^{2}\right)=0$

onde $a_{1}, a_{2}$ e $a_{3}$ são as componentes do versor normal ao plano de isotropia transversal; $\gamma_{1}$ e $\gamma_{2}$ são tais que $\|\mathbf{N}\|=1$.

Por simplificação, restringindo-se o estudo ao caso em que o sistema local de referência adotado para o material é aquele obtido impondo-se a direção 1 como sendo perpendicular ao plano de isotropia transversal, as componentes $a_{2}$ e $a_{3}$ resultam nulas e $a_{1}=1$. Nessas condições, as relações (3.26) assumem as seguintes formas:

$$
\begin{aligned}
& \|\mathbf{N}\|=1 \quad \Leftrightarrow \quad 3 \gamma_{1}^{2}+2 \gamma_{1} \gamma_{2}+\gamma_{2}^{2}=1 \\
& g(\varepsilon)=\gamma_{1} \operatorname{tr}\left(\varepsilon^{\mathbf{e}}\right)+\gamma_{2} \varepsilon_{11}^{\mathbf{e}}=0 \Rightarrow \quad \gamma_{1} \varepsilon_{\mathrm{V}}^{\mathrm{e}}+\gamma_{2} \varepsilon_{11}^{\mathbf{e}}=0
\end{aligned}
$$

De imediato, dois casos particulares merecem destaque:

- No primeiro caso, se $\gamma_{1}=1$ e $\gamma_{2}=0$, recupera-se a isotropia dada pelas equações (3.24) e (3.25). 
- no segundo caso, se $\gamma_{1}=0$ e $\gamma_{2}=1$, tem-se isotropia transversal pura e a normal ao hiperplano é coincidente com a normal ao plano de isotropia transversal do material. Segue, então que:

$g(\varepsilon)=\varepsilon_{11}^{\mathrm{e}}=0$

Este seria o hiperplano mais simples possível.

Voltando ao caso em estudo onde a resposta bimodular e isotropia transversal são induzidas pela danificação, ainda são válidas: $0 \leq \gamma_{1} \leq 1$ e $0 \leq \gamma_{2} \leq 1$. Pode-se supor, entretanto, que os coeficientes $\gamma_{1}$ e $\gamma_{2}$ sejam funções das variáveis de danificação.

Dessa maneira, segundo a forma dada pela (3.22), e em correspondência aos casos mais gerais de solicitação propõe-se para o hiperplano a seguinte relação:

$\mathrm{g}\left(\varepsilon, \mathbf{D}_{\mathbf{T}}, \mathbf{D}_{\mathbf{C}}\right)=\mathbf{N}\left(\mathbf{D}_{\mathbf{T}}, \mathbf{D}_{\mathbf{C}}\right) \cdot \varepsilon^{\mathbf{e}}=\gamma_{1}\left(\mathrm{D}_{1}, \mathrm{D}_{2}\right) \varepsilon_{\mathrm{V}}^{\mathrm{e}}+\gamma_{2}\left(\mathrm{D}_{1}, \mathrm{D}_{2}\right) \varepsilon_{11}^{\mathrm{e}}$

onde $\gamma_{1}\left(\mathrm{D}_{1}, \mathrm{D}_{2}\right)=\left\{1+\mathrm{H}\left(\mathrm{D}_{2}\right)\left[\mathrm{H}\left(\mathrm{D}_{1}\right)-1\right]\right\} \eta\left(\mathrm{D}_{1}\right)+\left\{1+\mathrm{H}\left(\mathrm{D}_{1}\right)\left[\mathrm{H}\left(\mathrm{D}_{2}\right)-1\right]\right\} \eta\left(\mathrm{D}_{2}\right)$ e $\gamma_{2}\left(\mathrm{D}_{1}, \mathrm{D}_{2}\right)$ $=\mathrm{D}_{1}+\mathrm{D}_{2}$.

As funções de Heaveside empregadas na última relação são dadas por:

$\begin{array}{llll}H\left(D_{i}\right)=1 & \text { para } & D_{i}>0 & \\ H\left(D_{i}\right)=0 & \text { para } & D_{i}=0 & (i=1,2)\end{array}$

As funções $\eta\left(D_{1}\right)$ e $\eta\left(D_{2}\right)$ são definidas, respectivamente, para os casos de tração, supondo que não tenha danificação prévia de compressão, e de compressão, supondo que não tenha havido danificação prévia de tração.

Por exemplo, em correspondência a estados de tração sem danificação prévia em compressão, a relação (3.30) reduz-se à seguinte equação representativa do hiperplano:

$\eta\left(\mathrm{D}_{1}\right) \varepsilon_{\mathrm{V}}^{\mathrm{e}}+\mathrm{D}_{1} \varepsilon_{11}^{\mathrm{e}}=0$ 
a qual pode ser usada para identificar a passagem para estados de compressão.

A função $\gamma_{1}=\eta\left(D_{1}\right)$ pode ser obtida da condição (3.27):

$\eta\left(D_{1}\right)=\frac{-D_{1}+\sqrt{3-2 D_{1}^{2}}}{3}$

Por outro lado, para estados de compressão sem danificação prévia em tração, a relação (3.30) passa a ser dada por:

$\eta\left(\mathrm{D}_{2}\right) \varepsilon_{\mathrm{V}}^{\mathrm{e}}+\mathrm{D}_{2} \varepsilon_{11}^{\mathrm{e}}=0$

Analogamente ao caso anterior, impondo-se a (3.27) resulta:

$\eta\left(D_{2}\right)=\frac{-D_{2}+\sqrt{3-2 D_{2}^{2}}}{3}$

Nota-se que não havendo danificação no material $\left(\mathrm{D}_{1}=\mathrm{D}_{2}=0\right)$ a (3.30) recupera a (3.25), satisfazendo assim a condição proposta de material inicialmente isótropo. Já para o material totalmente danificado, $D_{1}=D_{2}=1\left(\eta\left(D_{1}\right)=\eta\left(D_{2}\right)=0\right)$ e $\gamma_{2}=2$, recupera-se a (3.29).

Como exemplo, pode-se visualizar o hiperplano $\mathrm{g}(\boldsymbol{\varepsilon})$ nas duas situações descritas no parágrafo anterior. No caso de material íntegro, sem danificação, o hiperplano é dado pela equação (3.25) com sua normal possuindo iguais inclinações em relação aos eixos 1,2 e 3 sendo dada pelo tensor identidade de segunda ordem $\mathbf{I}$.

Já no caso de material totalmente danificado, o hiperplano $g(\varepsilon)$ (Figura 3.1) é coincidente com o plano de isotropia transversal local do material e, portanto, a normal ao hiperplano é dada pelo tensor de isotropia transversal $\mathbf{A}$. 


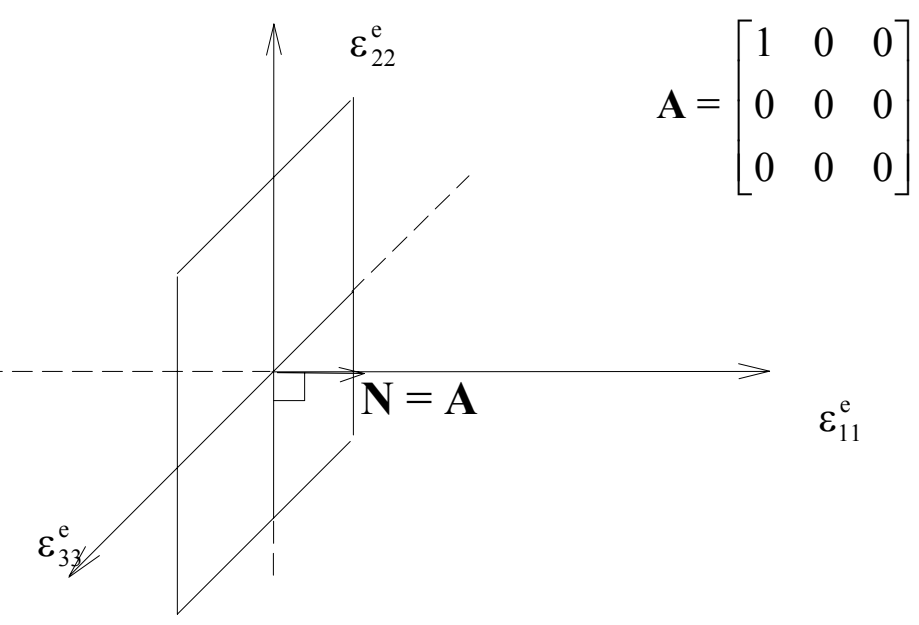

Figura 3.1 - Hiperplano g(ع) para o caso de material totalmente danificado.

Nos casos onde o material está sob um estado predominante de tração, mas que tenha sido ativado um processo de danificação anterior em compressão, o hiperplano (3.30) resulta em:

$\left(\eta\left(D_{1}\right)+\eta\left(D_{2}\right)\right) \varepsilon_{v}^{\mathrm{e}}+\left(D_{1}+D_{2}\right) \varepsilon_{11}^{\mathrm{e}}=0$

onde $\eta\left(D_{2}\right)$ e $D_{2}$ são nessa expressão constantes que registram a danificação previamente ocorrida em compressão. A mesma relação é válida para estados predominantes de compressão com ativação anterior do processo de danificação em tração; nesse caso, $\eta\left(D_{1}\right)$ e $D_{1}$ são os valores constantes que registram a danificação prévia em tração.

\subsection{CRITÉRIOS DE DANIFICAÇÃO}

Como visto, na formulação do modelo a danificação induz anisotropia no concreto. Sendo, portanto, o material inicialmente isótropo linear é conveniente separar os critérios de danificação em: critério para início de danificação, quando o material deixa de ser isótropo; e critério para carga e descarga, entendido aqui num sentido de evolução ou não das variáveis de dano, quando o material já apresentar-se 
como transversalmente isótropo. Ambos os critérios são baseados em energia de deformação.

\subsubsection{Critério para Início de Dano}

A ativação inicial dos processos de danificação é governada por uma limitação da quantidade de energia que pode ser movimentada em processos puramente reversíveis. Sugere-se como critério para identificação de início da danificação a comparação entre a energia de deformação elástica complementar $\mathrm{W}_{\mathrm{e}}^{*}$, calculada localmente considerando-se o meio inicialmente íntegro, isótropo e puramente elástico, e um certo valor de referência $\mathrm{Y}_{0 \mathrm{~T}}$, ou $\mathrm{Y}_{0 \mathrm{C}}$, obtido de ensaios uniaxiais de tração, ou de compressão, respectivamente.

Por exemplo, para estados predominantes de tração, caracterizados por $\mathrm{g}\left(\boldsymbol{\varepsilon}, \mathbf{D}_{\mathbf{T}}, \mathbf{D}_{\mathbf{C}}\right)$ $>0$, ver (3.30), se

$\mathrm{f}_{\mathrm{T}}(\sigma)=\mathrm{W}_{\mathrm{e}}^{*}-\mathrm{Y}_{0 \mathrm{~T}}<0$

então $\mathbf{D}_{\mathrm{T}}=\mathbf{0}$ ( ou seja, $\mathrm{D}_{1}=\mathrm{D}_{4}=0$ ) e o regime de resposta do material é elástico linear e isótropo.

Por outro lado, para estados predominantes de compressão, caracterizados por $\mathrm{g}\left(\varepsilon, \mathbf{D}_{\mathrm{T}}, \mathbf{D}_{\mathbf{C}}\right)<0$, ver (3.30), se

$\mathrm{f}_{\mathrm{C}}(\sigma)=\mathrm{W}_{\mathrm{e}}^{*}-\mathrm{Y}_{0 \mathrm{C}}<0$

então $\mathbf{D}_{\mathbf{C}}=\mathbf{0}$ (ou seja, $\mathrm{D}_{2}=\mathrm{D}_{3}=\mathrm{D}_{5}=0$ ) e o regime de resposta do material é elástico linear e isótropo.

Os valores de referência $\mathrm{Y}_{0 \mathrm{~T}}$ e $\mathrm{Y}_{0 \mathrm{C}}$ são parâmetros do modelo definidos pelas seguintes expressões:

$\mathrm{Y}_{0 \mathrm{~T}}=\frac{\sigma_{0 \mathrm{~T}}^{2}}{2 \mathrm{E}_{0}} \quad ; \quad \mathrm{Y}_{0 \mathrm{C}}=\frac{\sigma_{0 \mathrm{C}}^{2}}{2 \mathrm{E}_{0}}$ 
onde $\sigma_{0 \mathrm{~T}}$ e $\sigma_{0 \mathrm{C}}$ são as tensões dos limites elásticos determinados em regimes uniaxiais de tração e de compressão, respectivamente. Já a expressão para o cálculo de $\mathrm{W}_{\mathrm{e}}^{*}$ é dada por:

$$
\mathrm{W}_{\mathrm{e}}^{*}=-\frac{v_{0}}{2 \mathrm{E}_{0}} \operatorname{tr}^{2}(\sigma)+\frac{1+\mathrm{v}_{0}}{2 \mathrm{E}_{0}} \operatorname{tr}\left(\sigma^{2}\right)
$$

As condições que indicam o início de danificação podem ser expressas pelas relações de complementaridade:

$$
\begin{aligned}
& \mathrm{f}_{\mathrm{T}} \dot{\mathbf{D}}_{\mathrm{T}}=0 \\
& \mathrm{f}_{\mathrm{C}} \dot{\mathbf{D}}_{\mathrm{C}}=0
\end{aligned}
$$

onde $\dot{\mathbf{D}}_{\mathrm{T}}$ e $\dot{\mathbf{D}}_{\mathrm{C}}$ representam taxas de dano em tração e em compressão, respectivamente.

\subsubsection{Critério de Carregamento-Descarregamento do Meio Danificado}

É importante notar que o meio danificado apresenta um plano de isotropia transversal em correspondência ao nível atual de dano. Uma proposta para a localização desse plano será apresentada mais adiante. No que segue, por conveniência, admite-se que seja conhecido o plano de isotropia transversal e que tenha sido adotado um sistema de coordenadas locais tal que a direção 1 seja perpendicular àquele plano.

A energia elástica complementar do meio danificado expressa-se segundo formas diferentes, dependendo se os estados de deformação predominantes são de tração ou de compressão. Para o caso de estados predominantes de tração $\left(\mathrm{g}\left(\varepsilon, \mathbf{D}_{\mathbf{T}}, \mathbf{D}_{\mathbf{C}}\right)>0\right)$ vale a relação: 


$$
\begin{aligned}
\mathrm{W}_{\mathrm{e}+}^{*}= & \frac{\sigma_{11}^{2}}{2 \mathrm{E}_{0}\left(1-\mathrm{D}_{1}\right)^{2}}+\frac{\left(\sigma_{22}^{2}+\sigma_{33}^{2}\right)}{2 \mathrm{E}_{0}}-\frac{v_{0}\left(\sigma_{11} \sigma_{22}+\sigma_{11} \sigma_{33}\right)}{\mathrm{E}_{0}\left(1-\mathrm{D}_{1}\right)}-\frac{v_{0} \sigma_{22} \sigma_{33}}{\mathrm{E}_{0}} \\
& +\frac{\left(1+v_{0}\right)}{\mathrm{E}_{0}\left(1-\mathrm{D}_{4}\right)^{2}\left(1-\mathrm{D}_{5}\right)^{2}}\left(\sigma_{12}^{2}+\sigma_{13}^{2}\right)+\frac{\left(1+v_{0}\right)}{\mathrm{E}_{0}} \sigma_{23}^{2}
\end{aligned}
$$

Por outro lado, para estados predominantes de compressão $\left(g\left(\varepsilon, \mathbf{D}_{\mathbf{T}}, \mathbf{D}_{\mathbf{C}}\right)<0\right)$, a energia elástica complementar para um material com isotropia transversal induzida pelo dano é expressa por:

$$
\begin{aligned}
\mathrm{W}_{\mathrm{e}-}^{*}= & \frac{\sigma_{11}^{2}}{2 \mathrm{E}_{0}\left(1-\mathrm{D}_{2}\right)^{2}}+\frac{\left(\sigma_{22}^{2}+\sigma_{33}^{2}\right)}{2 \mathrm{E}_{0}\left(1-\mathrm{D}_{3}\right)^{2}}-\frac{v_{0}\left(\sigma_{11} \sigma_{22}+\sigma_{11} \sigma_{33}\right)}{\mathrm{E}_{0}\left(1-\mathrm{D}_{2}\right)\left(1-\mathrm{D}_{3}\right)}-\frac{v_{0} \sigma_{22} \sigma_{33}}{\mathrm{E}_{0}\left(1-\mathrm{D}_{3}\right)^{2}} \\
& +\frac{\left(1+v_{0}\right)}{\mathrm{E}_{0}\left(1-\mathrm{D}_{4}\right)^{2}\left(1-\mathrm{D}_{5}\right)^{2}}\left(\sigma_{12}^{2}+\sigma_{13}^{2}\right)+\frac{\left(1+v_{0}\right)}{\mathrm{E}_{0}} \sigma_{23}^{2}
\end{aligned}
$$

Considerando-se, então, uma situação geral de meio danificado em regime predominante de tração, o critério para a identificação de acréscimos de danificação é representado pela seguinte relação:

$\mathrm{f}_{\mathrm{T}}(\sigma)=\mathrm{W}_{\mathrm{e}+}^{*}-\mathrm{Y}_{0 \mathrm{~T}}^{*} \leq 0$

onde o valor de referência $\mathrm{Y}_{0 \mathrm{~T}}^{*}$ é definido pela máxima energia elástica complementar determinada ao longo do processo de danificação até o estado atual, isto é:

$\mathrm{Y}_{0 \mathrm{~T}}^{*}=\operatorname{MAX}\left(\mathrm{Y}_{0 \mathrm{~T}}^{*}, \mathrm{~W}_{\mathrm{e}+}^{*}\right)$

As condições teóricas de carregamento-descarregamento ficam então dadas pelas seguintes relações:

$\mathrm{f}_{\mathrm{T}} \leq 0, \quad \dot{\mathbf{D}}_{\mathrm{T}} \geq \mathbf{0}, \quad \mathrm{f}_{\mathrm{T}} \dot{\mathbf{D}}_{\mathrm{T}}=\mathbf{0}$

Se $\mathrm{f}_{\mathrm{T}}=0, \quad \dot{\mathrm{f}}_{\mathrm{T}} \dot{\mathbf{D}}_{\mathrm{T}}=\mathbf{0}$ 
Já para uma situação geral de meio danificado em regime predominante de compressão, o critério para a identificação de acréscimos de danificação é representado pela seguinte relação:

$\mathrm{f}_{\mathrm{C}}(\sigma)=\mathrm{W}_{\mathrm{e}_{-}}^{*}-\mathrm{Y}_{0 \mathrm{C}}^{*} \leq 0$

Analogamente ao caso de tração, o valor de referência $\mathrm{Y}_{\mathrm{OC}}^{*}$ resulta de:

$\mathrm{Y}_{0 \mathrm{C}}^{*}=\operatorname{MAX}\left(\mathrm{Y}_{0 \mathrm{C}}^{*}, \mathrm{~W}_{\mathrm{e}_{-}}^{*}\right)$

As condições teóricas de carregamento-descarregamento passam a ser dadas por:

$\mathrm{f}_{\mathrm{C}} \leq 0, \quad \dot{\mathbf{D}}_{\mathrm{C}} \geq \mathbf{0}, \quad \mathrm{f}_{\mathrm{C}} \dot{\mathbf{D}}_{\mathrm{C}}=\mathbf{0}$

Se $\mathrm{f}_{\mathrm{C}}=0, \quad \dot{\mathrm{f}}_{\mathrm{C}} \dot{\mathbf{D}}_{\mathrm{C}}=\mathbf{0}$

Uma situação particular importante que decorre deste critério é que existe a possibilidade de haver danificação evolutiva em compressão hidrostática.

Nos casos onde configura-se carregamento, isto é, onde $\dot{\mathbf{D}}_{\mathrm{T}} \neq \mathbf{0}$ ou $\dot{\mathbf{D}}_{\mathrm{C}} \neq \mathbf{0}$, é necessário atualizar os valores das variáveis escalares de dano que aparecem nos tensores $\mathbf{D}_{\mathbf{T}}$ e $\mathbf{D}_{\mathbf{C}}$, considerando-se suas leis de evolução.

Neste trabalho, propõe-se que as leis de evolução das variáveis de dano sejam escritas em função de suas variáveis associadas, as quais, correspondem à energia liberada durante o processo de evolução do dano, LEMAITRE (1996).

Dos potenciais de energia complementar (3.42) e (3.43) decorrem as variáveis associadas ao dano. Por exemplo, a expressão para a energia liberada durante o processo de evolução do dano, em estados predominantes de tração, é dada por:

$\mathrm{Y}_{\mathrm{T}}=\frac{\partial \mathrm{W}_{\mathrm{e}+}^{*}}{\partial \mathrm{D}_{1}}+\frac{\partial \mathrm{W}_{\mathrm{e}+}^{*}}{\partial \mathrm{D}_{4}}=\mathrm{Y}_{1}+\mathrm{Y}_{4}$ 
onde:

$$
\begin{aligned}
& Y_{1}=\frac{\sigma_{11}^{2}}{E_{0}\left(1-D_{1}\right)^{3}}-\frac{v_{0}\left(\sigma_{11} \sigma_{22}+\sigma_{11} \sigma_{33}\right)}{E_{0}\left(1-D_{1}\right)^{2}} \\
& Y_{4}=\frac{\left(1+v_{0}\right)}{E_{0}\left(1-D_{4}\right)^{3}\left(1-D_{5}\right)^{2}}\left(2 \sigma_{12}^{2}+2 \sigma_{13}^{2}\right)
\end{aligned}
$$

As variáveis $Y_{1}$ e $Y_{4}$ poderão ser incorporadas, respectivamente, nas leis de evolução das variáveis de dano $\mathrm{D}_{1}$ e $\mathrm{D}_{4}$.

Já a energia liberada durante o processo de evolução do dano em estados predominantes de compressão resulta:

$\mathrm{Y}_{\mathrm{C}}=\frac{\partial \mathrm{W}_{\mathrm{e}-}^{*}}{\partial \mathrm{D}_{2}}+\frac{\partial \mathrm{W}_{\mathrm{e}-}^{*}}{\partial \mathrm{D}_{3}}+\frac{\partial \mathrm{W}_{\mathrm{e}-}^{*}}{\partial \mathrm{D}_{5}}=\mathrm{Y}_{2}+\mathrm{Y}_{3}+\mathrm{Y}_{5}$

onde:

$$
\begin{aligned}
& Y_{2}=\frac{\sigma_{11}^{2}}{E_{0}\left(1-D_{2}\right)^{3}}-\frac{v_{0}\left(\sigma_{11} \sigma_{22}+\sigma_{11} \sigma_{33}\right)}{E_{0}\left(1-D_{2}\right)^{2}\left(1-D_{3}\right)} \\
& Y_{3}=\frac{\left(\sigma_{22}^{2}+\sigma_{33}^{2}\right)}{E_{0}\left(1-D_{3}\right)^{3}}-\frac{v_{0}\left(\sigma_{11} \sigma_{22}+\sigma_{11} \sigma_{33}\right)}{E_{0}\left(1-D_{2}\right)\left(1-D_{3}\right)^{2}}-\frac{2 v_{0} \sigma_{22} \sigma_{33}}{E_{0}\left(1-D_{3}\right)^{2}} \\
& Y_{5}=\frac{\left(1+v_{0}\right)}{E_{0}\left(1-D_{5}\right)^{3}\left(1-D_{4}\right)^{2}}\left(2 \sigma_{12}^{2}+2 \sigma_{13}^{2}\right)
\end{aligned}
$$

As variáveis $\mathrm{Y}_{2}, \mathrm{Y}_{3}$ e $\mathrm{Y}_{5}$ poderão ser incorporadas, respectivamente, nas leis de evolução das variáveis de dano $\mathrm{D}_{2}, \mathrm{D}_{3}$ e $\mathrm{D}_{5}$. Observa-se em particular que no caso de compressão hidrostática resulta um valor nulo para $Y_{5}$, pois as tensões de cisalhamento são nulas. Nesse caso, a evolução de $\mathrm{D}_{5}$ não ocorre. Nota-se, portanto, que o critério de evolução do dano pode indicar a possibilidade de variação do tensor correspondente, o que não necessariamente implica em evolução de todas as variáveis de danificação nele contidas. 
De um modo geral, nota-se que as relações que definem as variáveis associadas podem ser representadas por:

$\mathrm{Y}_{\mathrm{T}, \mathrm{C}}=\mathrm{F}\left(\sigma, \mathbf{E}_{\mathbf{0}}, \mathbf{D}_{\mathrm{T}, \mathbf{C}}\right)$

Também usando de uma representação implícita, as leis de evolução das variáveis de dano podem ser dadas por:

$\dot{\mathbf{D}}_{\mathrm{T}, \mathrm{C}}=\mathrm{F}^{*}\left(\mathrm{Y}_{\mathrm{T}, \mathrm{C}}, \mathrm{b}_{\mathrm{T}, \mathrm{C}}\right)$

onde $b_{T, C}$ são conjuntos de parâmetros contidos nas leis de evolução de $\mathbf{D}_{\mathbf{T}}$ ou $\mathbf{D}_{\mathbf{C}}$. Observa-se que para situações de carregamento monotônico crescente, as relações (3.58) podem ser integradas diretamente. Entretanto, as relações para $\mathrm{Y}_{\mathrm{T}, \mathrm{C}}$ e $\mathbf{D}_{\mathrm{T}, \mathrm{C}}$ formam um sistema implícito. A solução do sistema pode ser obtida por meio de um procedimento iterativo.

\subsection{LEIS DE EVOLUÇÃO DE DANO}

Finalmente, observa-se que as leis de evolução das variáveis de dano em tração $\dot{\mathrm{D}}_{1}, \dot{\mathrm{D}}_{4}$ e em compressão $\dot{\mathrm{D}}_{2}, \dot{\mathrm{D}}_{3}$ e $\dot{\mathrm{D}}_{5}$, constituem, talvez, o aspecto mais arbitrário da modelagem.

Em geral, as leis de evolução das variáveis de dano podem ser obtidas pelas seguintes estratégias:

- por identificação direta a partir de respostas experimentais obtidas em laboratórios;

- pela definição de um potencial de dissipação escrito em função das variáveis associadas, que engloba estados que podem ser atingidos sem qualquer dissipação adicional de energia e de cuja variação derivam as leis de evolução. 
Um inconveniente da primeira estratégia limitante de sua generalidade é que ela pode conduzir a leis que tenham aplicação restrita a combinações de solicitações similares àquelas dos ensaios realizados.

A segunda estratégia tem um cunho mais teórico, porém com algumas vantagens tais como: aplicação a situações gerais de solicitações, estrutura formal do modelo resultante bastante próxima daquela obtida por outras teorias e possibilidade de manutenção de simetria do tensor constitutivo.

Limitando-se a análise ao caso de carregamento monotônico crescente, as leis de evolução propostas para as variáveis escalares de dano são resultantes de ajustes sobre resultados experimentais e apresentam características similares àquelas encontradas nos trabalhos de MAZARS (1984) e LA BORDERIE (1991). A forma geral proposta é a seguinte:

$$
D_{i}=1-\frac{1+A_{i}}{A_{i}+\exp \left[B_{i}\left(Y_{i}-Y_{0 i}\right)\right]} \quad \operatorname{com} i=1,5
$$

onde $A_{i}, B_{i}$ e $Y_{0 i}$ são parâmetros a serem identificados. $O$ sub-índice $i$ está relacionado à variável de dano escalar que está sendo calculada. Os parâmetros $\mathrm{Y}_{0 \mathrm{i}}$ são entendidos como limites iniciais para a ativação da danificação, os mesmos utilizados nas Eqs. (3.37) e (3.38).

A identificação paramétrica do modelo é realizada através de ensaios experimentais de tração uniaxial (para a obtenção dos parâmetros $A_{1}, B_{1}$ e $Y_{01}=$ $\mathrm{Y}_{0 \mathrm{~T}}$ ), de ensaios de compressão uniaxial (para a identificação dos parâmetros $\mathrm{A}_{2}, \mathrm{~B}_{2}$ e $\mathrm{Y}_{02}=\mathrm{Y}_{0 \mathrm{C}}$ ) e finalmente de ensaios de compressão biaxial (para obtenção de $\mathrm{A}_{3}, \mathrm{~B}_{3}$ e $\mathrm{Y}_{03}=\mathrm{Y}_{02}=\mathrm{Y}_{0 \mathrm{C}}$ ). Esses ensaios experimentais uniaxiais e biaxiais serão ilustrados mais adiante. Por outro lado, a identificação dos parâmetros contidos nas leis de evolução correspondentes às variáveis de dano $\mathrm{D}_{4}$ e $\mathrm{D}_{5}$, as quais influenciam o comportamento do concreto em cisalhamento, não será objeto de estudo neste trabalho, por não estarem disponíveis ensaios que permitam a calibração dos parâmetros ou, até mesmo, a proposição de leis de evolução mais realistas. Tais ensaios são complexos, pois exigem a necessidade de se criar um estado de cisalhamento puro. No entanto, permitiriam a obtenção de uma curva tensão de 
cisalhamento $\mathrm{x}$ distorção a ser empregada tanto na proposição de leis de evolução de dano, quanto para a identificação paramétrica do modelo.

\subsection{CRITÉRIO PARA A DEFINIÇÃO DO PLANO LOCAL DE ISOTROPIA TRANSVERSAL DO MATERIAL}

Quando o processo de danificação é ativado, o modelo proposto passa a envolver o tensor A que, por sua vez, depende do conhecimento da normal ao plano de isotropia transversal. Torna-se necessário, portanto, definir regras para a localização da normal àquele plano para um dado estado de deformação.

Inicialmente estabelece-se um critério geral para a existência do plano de isotropia transversal. Propõe-se, neste trabalho, que a isotropia transversal decorrente da danificação se manifesta somente se existirem taxas positivas de deformação, ao menos em uma das direções principais. Decorre dessa hipótese que em compressão hidrostática o material mantém-se isótropo.

Estabelecido o critério geral para existência do plano, definem-se algumas regras para identificar sua localização.

Imaginando-se, em primeiro lugar, um estado de deformação em que uma das taxas de deformação é não-nula ou de sinal contrário às demais, aplica-se a seguinte regra:

"No espaço das deformações principais, se duas das três taxas de deformação forem de alongamento, de encurtamento ou nulas, o plano definido por elas será o plano local de isotropia transversal do material."

Nesse caso enquadra-se, por exemplo, a tração uniaxial; resultando que o plano de isotropia transversal é perpendicular à direção da tensão de tração.

Há casos não abrangidos por essa regra. Por exemplo, o estado de deformação plana em que as deformações não-nulas são de sinais contrários. Com a primeira regra proposta torna-se impossível identificar o plano local de isotropia transversal do material. Para essa situação, vale uma segunda regra: 
"Num estado de deformação plana, onde as taxas de deformação principal no plano tenham sinais contrários, o plano local de isotropia transversal do material fica definido pelas direções da deformação principal permanentemente nula e da deformação cuja taxa seja positiva."

Um outro caso particular ocorre quando todas as taxas de deformação principal são positivas. Para esses estados vale uma terceira regra, segundo a qual assume-se que a direção de maior alongamento seja perpendicular ao plano local de isotropia transversal do material.

Cabe observar que as regras propostas foram concebidas de modo a englobar a quase totalidade dos estados de deformação observados experimentalmente. Eventuais estados que não se incluem nas regras propostas são, por convenção, estados em que a isotropia transversal não se manifesta e, nesses casos, o tensor $\mathbf{A}$ resulta igual à identidade.

\subsection{PROPOSTA DE MODELO DE DANO COM DEFORMAÇÕES ANELÁSTICAS - VERSÃO UNIAXIAL}

A proposta original do modelo de dano apresentado neste trabalho, por simplificação, não leva em conta as deformações residuais. Segundo o modelo proposto, portanto, quando se efetua um descarregamento, recupera-se totalmente o valor da deformação. 


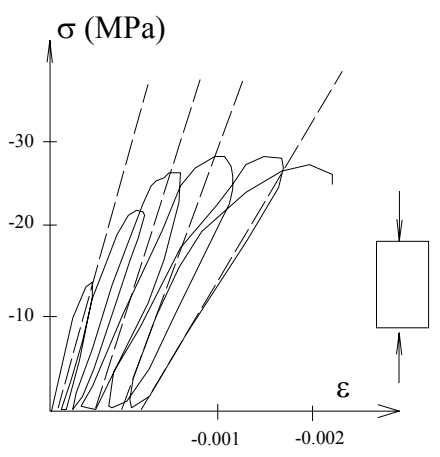

A)

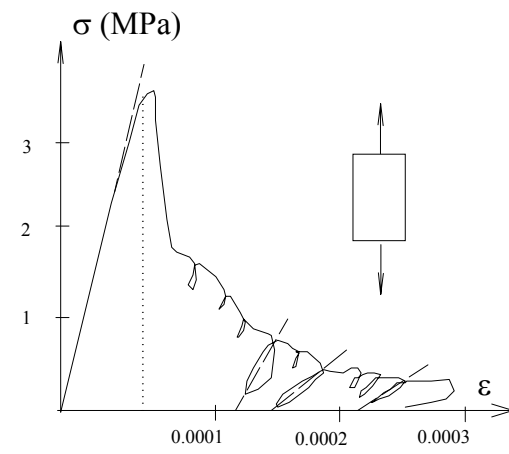

B)

Figura 3.2 - Diagramas tensão-deformação em compressão e tração uniaxiais

[MAZARS (1984)]

No entanto, como mostra a figura 3.2, observações experimentais indicam que as deformações permanentes não são desprezíveis nas situações de descarregamento. Alguns modelos de dano levam em conta na sua formulação tais deformações associando-as exclusivamente ao fenômeno da danificação. O tipo de acoplamento adotado normalmente é o cinemático, o início e evolução de microfissuras influenciam na velocidade das deformações permanentes. Neste contexto, pode-se citar os modelos propostos por COMI (2000), PAPA \& TALIERCIO (1996), RAMTANI (1990), entre outros.

De fato, as deformações anelásticas e a danificação evoluem simultaneamente. Segundo LA BORDERIE (1991), a microfissuração e a existência de vazios no material estão na origem dos dois fenômenos. Por um lado os microvazios são uma causa para as deformações anelásticas, pois impedem que as microfissuras fechem totalmente num processo de descarregamento. Por outro lado, nota-se numa microfissura que suas faces são irregulares, sendo esta uma outra razão para a dificuldade do seu fechamento total.

Neste item trata-se da incorporação de deformações residuais no modelo constitutivo proposto para o concreto danificado. Como critério geral, admite-se que tais deformações surgem após o início da danificação. Limita-se a proposta apenas os casos uniaxiais, pois tratando-se de um modelo de dano com anisotropia induzida, as deformações plásticas em estados mais complexos de solicitação devem ser melhor avaliadas. 
Considere-se a resposta idealizada na figura 3.3, representando duas etapas de descarregamento num ensaio de compressão uniaxial. Evidencia-se nessas etapas, o módulo de elasticidade $\mathrm{E}$ danificado de valor inferior ao módulo $\mathrm{E}_{0}$ do material íntegro. Naturalmente, os módulos de elasticidade E para as várias situações dependem do dano acumulado.

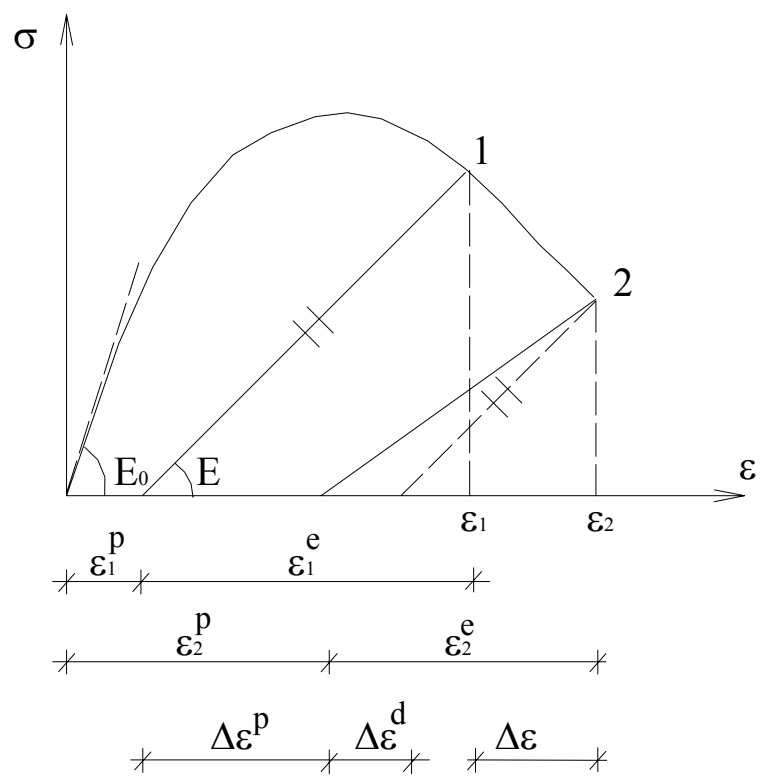

Figura 3.3 - Diagrama tensão-deformação em compressão uniaxial com carga e descarga

Tendo-se em vista o diagrama tensão-deformação idealizado, vale a seguinte relação:

$\varepsilon=\varepsilon^{\mathrm{e}}+\varepsilon^{\mathrm{p}}$

ou ainda, em termos de taxas de deformação:

$\dot{\varepsilon}=\dot{\varepsilon}^{\mathrm{e}}+\dot{\varepsilon}^{\mathrm{p}}$

onde $\varepsilon, \varepsilon^{\mathrm{e}}, \varepsilon^{\mathrm{p}}$ são, respectivamente, a deformação total, a parcela de deformação elástica e a parcela de deformação plástica correspondentes a uma dada tensão $\sigma$.

Segundo a figura 3.3, a deformação elástica $\varepsilon^{\mathrm{e}}$ pode ser obtida da seguinte relação: 
$\varepsilon^{\mathrm{e}}=\frac{\sigma}{\mathrm{E}}=\mathrm{C} \sigma$

onde $\mathrm{E}$ e C são, respectivamente, os módulos de rigidez e de flexibilidade do material danificado.

Em termos de taxas, a (3.62) passa a ser escrita como:

$\dot{\varepsilon}^{\mathrm{e}}=\dot{\mathrm{C}} \sigma+\mathrm{C} \dot{\sigma}$

onde $\dot{C} \sigma$ corresponde à parcela da taxa de deformação elástica devida à danificação e representada por $\dot{\varepsilon}^{\mathrm{d}}$. Logo,

$\dot{\varepsilon}^{\mathrm{e}}=\left.\dot{\varepsilon}^{\mathrm{e}}\right|_{\mathrm{C}=\text { cte }}+\dot{\varepsilon}^{\mathrm{d}}$

Substituindo-se a (3.64) na (3.61), resulta:

$\dot{\varepsilon}=\left.\dot{\varepsilon}^{\mathrm{e}}\right|_{\mathrm{C}=\text { cte }}+\dot{\varepsilon}^{\mathrm{d}}+\dot{\varepsilon}^{\mathrm{p}}$

Sendo a taxa de deformação elástica $\left.\dot{\varepsilon}^{\mathrm{e}}\right|_{\mathrm{C}=\text { cte }}$ função do nível atual de dano, e $\dot{\varepsilon}^{\mathrm{d}}$ dependente também da taxa de danificação, essas relações de dependência podem ser representadas por:

$\dot{\varepsilon}^{\mathrm{d}}=\dot{\varepsilon}^{\mathrm{d}}(\mathrm{D}, \dot{\mathrm{D}})$

$\left.\dot{\varepsilon}^{\mathrm{e}}\right|_{\mathrm{C}=\mathrm{cte}}=\dot{\varepsilon}^{\mathrm{e}}(\mathrm{D})$

Sendo, por hipótese, a deformação plástica dependente do processo de danificação, obtém-se para um dado $\dot{\varepsilon}$ que: 


$$
\dot{\varepsilon}^{\mathrm{p}}=\dot{\varepsilon}^{\mathrm{p}}(\mathrm{D}, \dot{\mathrm{D}})
$$

Por outro lado, da (3.65) resultam:

$$
\begin{aligned}
& \dot{\varepsilon}^{\mathrm{p}}=\dot{\varepsilon}-\left.\dot{\varepsilon}^{\mathrm{e}}\right|_{\mathrm{C}=\text { cte }}-\dot{\varepsilon}^{\mathrm{d}} \\
& \dot{\varepsilon}^{\mathrm{p}}=\left.\dot{\varepsilon}^{\mathrm{p}}\right|_{\mathrm{C}=\text { cte }}-\dot{\varepsilon}^{\mathrm{d}}
\end{aligned}
$$

A expressão (3.70) indica que para se determinar $\dot{\varepsilon}^{\mathrm{p}}$ pode-se realizar uma análise elastoplástica usual com $\left.\dot{\varepsilon}^{\mathrm{p}}\right|_{\mathrm{C}=\text { cte }}\left(=\dot{\lambda} \mathrm{f}_{\sigma}\right)$ seguida de uma análise de dano para definir $\dot{\varepsilon}^{\mathrm{d}}$.

Voltando à caracterização da evolução das deformações plásticas, segundo a (3.68), pode-se estabelecer duas hipóteses simplificadoras: na primeira, a taxa de deformação plástica é função exclusiva do estado de danificação no instante da análise; na segunda, a taxa de deformação plástica depende diretamente da evolução da danificação do material.

No âmbito da primeira hipótese $\left(\dot{\varepsilon}^{\mathrm{p}}=\dot{\varepsilon}^{\mathrm{p}}(\mathrm{D})\right)$, pode-se estabelecer que à medida em que a danificação avança a taxa de evolução da deformação plástica tenda ao valor nulo $\left(\dot{\varepsilon}^{\mathrm{p}} \rightarrow 0\right.$ ), ou seja, $\dot{\varepsilon}^{\mathrm{d}}$ se aproxima de $\left.\dot{\varepsilon}^{\mathrm{p}}\right|_{\mathrm{C}=\text { cte }}$ segundo a equação (3.70). Fisicamente, pode-se relacionar esse caso com a situação em que a danificação destrói a resistência ao cisalhamento ('engrenamento') responsável pela deformação plástica. Numa situação de compressão uniaxial, o gráfico da figura seguinte ilustra a situação em questão. 


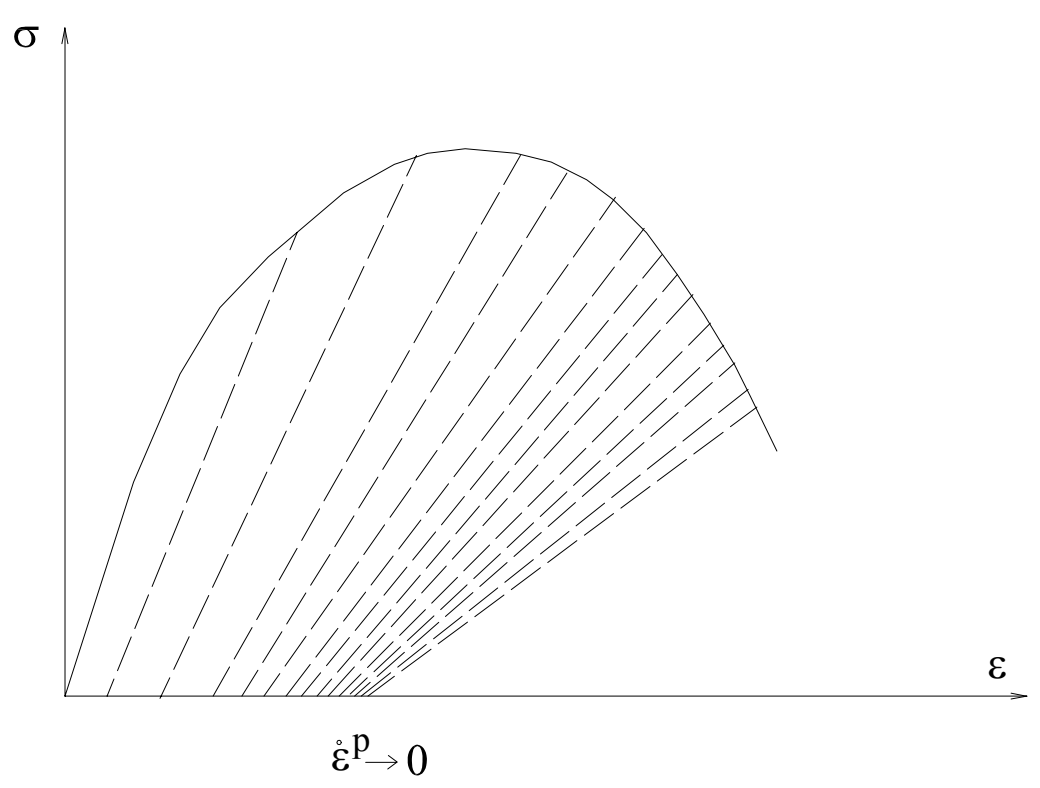

Figura 3.4 - Taxa de deformação plástica ( $\left(1^{\mathrm{a}}\right.$ Hipótese)

Nessas condições, e em conformidade com a quantificação do dano por equivalência de energia (algo mais simples poderia ser simplesmente $\dot{\varepsilon}^{\mathrm{d}}=\left.\mathrm{D} \dot{\varepsilon}^{\mathrm{p}}\right|_{\mathrm{C}=\text { cte }}$ ), pode-se propor a seguinte relação entre $\dot{\varepsilon}^{\mathrm{d}}$ e $\dot{\varepsilon}^{\mathrm{p}}$ :

$$
\dot{\varepsilon}^{\mathrm{d}}=\left.\left(2 \mathrm{D}-\mathrm{D}^{2}\right) \dot{\varepsilon}^{\mathrm{p}}\right|_{\mathrm{C}=\mathrm{cte}}
$$

Assim, segundo a (3.70) fornece:

$$
\dot{\varepsilon}^{\mathrm{p}}=\left.(1-\mathrm{D})^{2} \dot{\varepsilon}^{\mathrm{p}}\right|_{\mathrm{C}=\mathrm{cte}}=\dot{\varepsilon}^{\mathrm{p}}(\mathrm{D})
$$

Já na segunda hipótese $\left(\dot{\varepsilon}^{\mathrm{p}}=\dot{\varepsilon}^{\mathrm{p}}(\dot{\mathrm{D}})\right)$, a taxa de deformação plástica passa a ser função exclusiva da velocidade de danificação do material.

Um modo de satisfazer essa condição é estabelecer que:

$$
\left.\left.\dot{\varepsilon}^{\mathrm{p}}\right|_{\mathrm{C}=\mathrm{cte}} \cong \dot{\varepsilon}^{\mathrm{p}}\right|_{\mathrm{C}_{0}}
$$


onde $\mathrm{C}_{0}$ é o módulo de flexibilidade do material íntegro.

Segue, então, da (3.70) que

$\dot{\varepsilon}^{\mathrm{p}}=\left.\dot{\varepsilon}^{\mathrm{p}}\right|_{\mathrm{C}_{0}}-\dot{\varepsilon}^{\mathrm{d}}(\dot{\mathrm{D}})$

Além disso, deve-se estabelecer uma regra para $\dot{\varepsilon}^{\mathrm{d}}(\dot{\mathrm{D}})$. Por generalidade, pode-se assumir que a evolução da deformação devido ao dano seja dada por uma regra não-associativa do tipo:

$\dot{\varepsilon}^{\mathrm{d}}(\dot{\mathrm{D}})=\dot{\mathrm{D}}_{\sigma}$

onde g é um certo potencial representativo, por exemplo, da energia disponibilizada para evolução do dano.

Deve-se garantir que a expressão $\dot{\mathrm{D}}_{\sigma}$ substituída na expressão (3.70) gere $\dot{\varepsilon}^{\mathrm{p}} \geq 0$, pois trata-se de um processo dissipativo.

Dessa forma,

$\dot{\varepsilon}^{\mathrm{p}}=\left.\dot{\varepsilon}^{\mathrm{p}}\right|_{\mathrm{C}_{0}}-\left.\dot{\mathrm{D}} \mathrm{g}_{\sigma}\right|_{\mathrm{C}_{0}}$

Numa situação de solicitação uniaxial com carga-descarga, a figura a seguir ilustra o comportamento de $\dot{\varepsilon}^{\mathrm{p}}$ dado pela expressão (3.74).

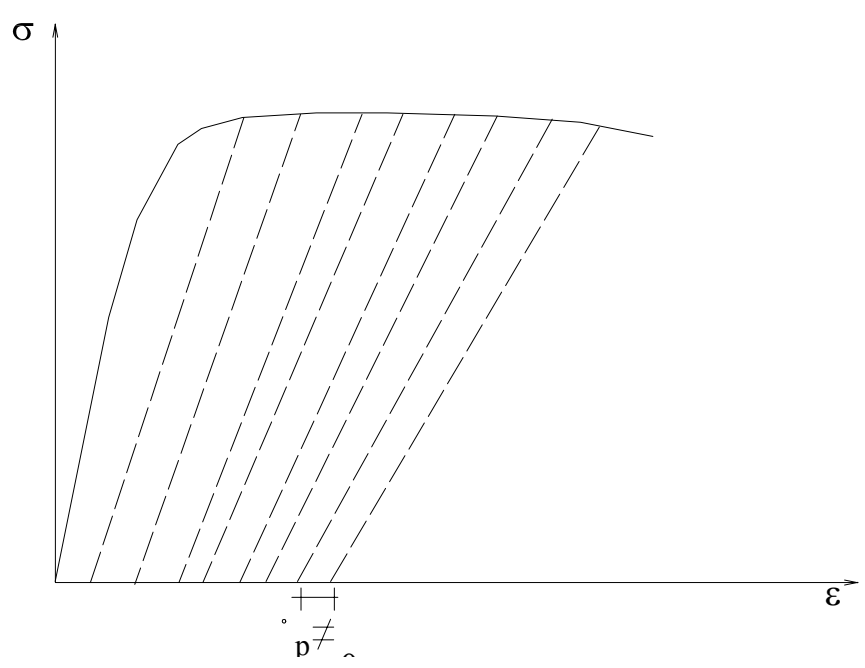

Figura 3.5 - Taxa de deformação plástica ( $\left(2^{\mathrm{a}}\right.$ Hipótese) 
Este tipo de análise parece ser bastante apropriado para a evolução das deformações plásticas em metais.

Os dois tratamentos simplificados não serão explorados independentemente. $\mathrm{Na}$ verdade eles servem de base para a proposição de uma regra direta para $\dot{\varepsilon}^{\mathrm{p}}$, considerando-se genericamente a dependência indicada pela (3.68).

Nesse contexto, e tendo-se em vista a bimodularidade, propõe-se uma lei de evolução não-associativa para as deformações residuais, inspirada na proposta de COMI (2000), dada por:

$\dot{\varepsilon}^{\mathrm{p}}=\frac{\partial \mathrm{g}_{\mathrm{T}}}{\partial \sigma} \dot{\mathbf{D}}_{\mathrm{T}}+\frac{\partial \mathrm{g}_{\mathrm{C}}}{\partial \sigma} \dot{\mathbf{D}}_{\mathrm{C}}$

onde $\mathrm{g}_{\mathrm{T}}$ e $\mathrm{g}_{\mathrm{C}}$ são potenciais anelásticos.

Admitindo-se, ainda por simplificação, que as deformações plásticas são compostas exclusivamente por deformações volumétricas, nos moldes de alguns modelos contidos na literatura [RAMTANI, BERTHAUD \& MAZARS (1992)], os potenciais podem ser expressos por:

$$
\begin{aligned}
& \mathrm{g}_{\mathrm{T}}=\beta_{\mathrm{T}}\left(\mathrm{D}_{\mathrm{T}}\right) \mathrm{I}_{1} \\
& \mathrm{~g}_{\mathrm{C}}=\beta_{\mathrm{C}}\left(\mathrm{D}_{\mathrm{C}}\right) \mathrm{I}_{1}
\end{aligned}
$$

sendo $\mathrm{I}_{1}$ o primeiro invariante do tensor de tensões.

Segue daí que a relação (3.77) assume a forma:

$$
\dot{\varepsilon}^{\mathrm{P}}=\beta_{\mathrm{T}}\left(\mathrm{D}_{\mathrm{T}}\right) \dot{\mathbf{D}}_{\mathrm{T}}+\beta_{\mathrm{C}}\left(\mathrm{D}_{\mathrm{C}}\right) \dot{\mathbf{D}}_{\mathrm{C}}
$$

As funções de dano $\beta_{\mathrm{T}}\left(\mathrm{D}_{\mathrm{T}}\right)$ e $\beta_{\mathrm{C}}\left(\mathrm{D}_{\mathrm{C}}\right)$, são dependentes do material. No caso do modelo proposto para aplicação ao concreto, assumem-se as seguintes funções:

$$
\beta_{\mathrm{T}}\left(\mathrm{D}_{\mathrm{T}}\right)=\frac{\beta_{1}}{\left(1-\mathrm{D}_{1}\right)^{2}}
$$


$\beta_{C}\left(D_{C}\right)=\frac{\beta_{2}}{\left(1-D_{2}\right)^{2}}$

Finalmente, a lei de evolução para as deformações plásticas levando-se em conta o efeito unilateral, resulta:

$$
\dot{\varepsilon}^{\mathrm{p}}=\left(\frac{\beta_{1}}{\left(1-\mathrm{D}_{1}\right)^{2}} \dot{\mathrm{D}}_{1}+\frac{\beta_{2}}{\left(1-\mathrm{D}_{2}\right)^{2}} \dot{\mathrm{D}}_{2}\right) \mathbf{I}
$$

Observa-se que nesta proposta, $\beta_{1}$ e $\beta_{2}$ são parâmetros diretamente relacionados às evoluções das deformações plásticas induzidas pelo dano em tração e em compressão, respectivamente. Quando os valores desses parâmetros são impostos como nulos, recupera-se o modelo originalmente proposto, ou seja, comportamento elástico-danificado sem a consideração de deformações plásticas.

A identificação e influência dos parâmetros $\beta_{1}$ e $\beta_{2}$ na resposta tensãodeformação estão descritas no próximo capítulo, assim como a influência de todos os outros parâmetros envolvidos na formulação do modelo proposto. 


\section{IDENTIFICAÇÃO PARAMÉTRICA DO MODELO PROPOSTO}

\subsection{INTRODUÇÃO}

A identificação paramétrica é essencial para a utilização de um modelo, e os valores podem apresentar grande sensibilidade principalmente nos casos onde o número de parâmetros é reduzido. Trata-se do caso do modelo proposto neste trabalho. A utilização de um número pequeno de parâmetros tem a vantagem de uma identificação menos complexa, entretanto possui a desvantagem de dificultar a captura integral das características da curva tensão-deformação do material que se pretende analisar. Por outro lado, para conseguir uma correta identificação e, conseqüentemente, um bom ajuste do modelo, é indispensável dispor de resultados experimentais confiáveis.

Neste capítulo são comentados o processo analítico utilizado na identificação paramétrica do modelo e as respostas esperadas em termos de curva tensãodeformação com a variação dos valores dos parâmetros. Para ilustrar as respostas fornecidas pelo modelo, a partir da calibração dos parâmetros contidos nas leis de evolução das variáveis de dano, apresentam-se alguns confrontos com ensaios experimentais uniaxiais, biaxiais e triaxiais no concreto. O objetivo desses confrontos é a validação do emprego do modelo para diversas combinações de solicitações. Finalmente, discutem-se algumas conclusões com relação às respostas numéricas obtidas. 


\subsection{PROCESSO ANALÍTICO DE IDENTIFICAÇÃO PARAMÉTRICA}

A modelagem analítica de um fenômeno físico consiste em assumir leis matemáticas representativas do fenômeno estudado experimentalmente. Essas oportunas leis dependem de um certo número de parâmetros cuja calibração (identificação) pressupõe a determinação dos valores ótimos para eles, de modo que a resposta numérica se aproxime o máximo possível da experimental.

O processo de identificação dos parâmetros característicos do modelo possui duas fases bem distintas. A primeira consiste na escolha de uma função erro, utilizada como um critério para a avaliação da resposta numérica em relação à experimental. Em termos intrínsecos a função erro pode ser indicada na forma:

$E=E\left(\mathrm{y}^{\mathrm{c}}(\mathrm{x}, \mathbf{a}), \mathrm{y}^{\mathrm{s}}(\mathrm{x})\right)=E(\mathbf{a})$

onde:

$\mathbf{a}^{\mathrm{T}}=\left[\mathrm{a}_{1}, \mathrm{a}_{2}, \ldots, \mathrm{a}_{\mathrm{n}}\right]$ é o vetor dos $\mathrm{n}$ parâmetros a serem identificados

$\mathrm{y}^{\mathrm{c}}(\mathrm{x}, \mathbf{a})$ é a resposta numérica, dependente do vetor de parâmetros a

$\mathrm{y}^{\mathrm{S}}(\mathrm{x})$ é a resposta experimental, podendo ser observada com continuidade num intervalo $\Delta \mathrm{x}$, ou registrada mediante pares de valores $\left[\mathrm{x}, \mathrm{y}^{\mathrm{s}}(\mathrm{x})\right]$.

A função erro é expressa por uma integral definida, ou uma somatória, sobre um intervalo de observação, por esse motivo não depende da variável x.

Numa formulação por mínimos quadrados, a função erro é genericamente definida pela integral dos quadrados da diferença entre as duas funções $y^{c}$ e $y^{s}$

$E\left(\mathrm{a}_{\mathrm{i}}\right)=\frac{1}{2} \int_{\Delta \mathrm{x}}\left[\mathrm{y}^{\mathrm{c}}\left(\mathrm{x}, \mathrm{a}_{\mathrm{i}}\right)-\mathrm{y}^{\mathrm{s}}(\mathrm{x})\right]^{2} \mathrm{dx}$

No caso de se conhecer a resposta experimental de modo discreto, a relação anterior assume a forma:

$E\left(\mathrm{a}_{\mathrm{i}}\right)=\frac{1}{2} \sum_{\mathrm{K}=1}^{\mathrm{N}}\left[\mathrm{y}_{\mathrm{k}}^{\mathrm{c}}\left(\mathrm{x}_{\mathrm{k}}, \mathrm{a}_{\mathrm{i}}\right)-\mathrm{y}_{\mathrm{k}}^{\mathrm{s}}\left(\mathrm{x}_{\mathrm{k}}\right)\right]^{2}$ 
A segunda fase do processo de identificação está relacionada à escolha do método de minimização da função erro. As variáveis do problema de otimização resultante são os próprios parâmetros do modelo.

Em resumo, o objetivo do problema de identificação é a determinação do vetor de parâmetros que fornece o menor erro, ou seja, uma resposta numérica que melhor descreva o comportamento experimental. Assim,

$E_{m}=\min _{\mathbf{a}}\{E\}$

\subsection{ANÁliSE DA INFLUÊNCIA PARAMÉTRICA DO MODELO NA RESPOSTA TENSÃO-DEFORMAÇÃO}

O estudo paramétrico será conduzido com referência a testes de tração e de compressão uniaxial e de compressão biaxial. Observa-se que esses ensaios são suficientes para a completa identificação dos parâmetros contidos nas leis de evolução das variáveis de dano da proposta de modelo. Apresenta-se também a influência dos parâmetros anelásticos $\beta_{1}$ e $\beta_{2}$ para a versão uniaxial do modelo com a adição de deformações residuais.

Os parâmetros a identificar podem ser agrupados da seguinte forma:

- parâmetros elásticos: $\mathrm{E}_{0}$ e $v_{0}$;

- parâmetros de danificação em tração: $\mathrm{Y}_{0 \mathrm{~T}}, \mathrm{~A}_{1}$ e $\mathrm{B}_{1}$;

- parâmetros de danificação em compressão: $\mathrm{Y}_{0 \mathrm{C}}, \mathrm{A}_{2}, \mathrm{~B}_{2}, \mathrm{~A}_{3}$ e $\mathrm{B}_{3}$;

- parâmetro anelástico em tração: $\beta_{1}$;

- parâmetro anelástico em compressão: $\beta_{2}$.

Os parâmetros elásticos são identificados em ensaios clássicos de compressão uniaxial.

Os parâmetros anelásticos $\beta_{1}$ e $\beta_{2}$ são identificados através de ensaios de tração e de compressão com deformação controlada, respectivamente, operando-se ciclos de carregamento e de descarregamento. Os parâmetros que aparecem nas leis de evolução das variáveis de dano podem ser identificados pelos mesmos ensaios, 
porém faz-se a ressalva que os parâmetros $A_{3}$ e $B_{3}$ são identificados através de ensaios de compressão biaxial.

É importante observar que os valores atribuídos para os parâmetros $\mathrm{A}_{\mathrm{i}}$ devem obedecer à limitação de $\mathrm{A}_{\mathrm{i}}>-1,0$. Isso pode ser explicado pela configuração da lei de evolução das variáveis de dano. Nota-se que para $A_{i} \leq-1,0$ logo de início um valor unitário seria assumido para a variável de dano corrente [ver capítulo 3, Eq. (3.59)], ou ainda a variável de dano poderia assumir valores maiores que a unidade.

Mas antes de ilustrar a identificação realizada sobre o modelo proposto, é interessante investigar a influência dos diferentes parâmetros na resposta tensãodeformação.

Com relação aos parâmetros de danificação em tração $\mathrm{Y}_{01}=\mathrm{Y}_{0 \mathrm{~T}}, \mathrm{~A}_{1}$ e $\mathrm{B}_{1}$, as figuras 4.1a, b e c ilustram a influência de cada um deles na relação tensãodeformação. Os ensaios numéricos de tração uniaxial foram realizados variando cada parâmetro a partir dos seguintes valores:

$$
\begin{aligned}
& \mathrm{E}_{0}=15600 \mathrm{MPa} \\
& \mathrm{Y}_{01}=\mathrm{Y}_{0 \mathrm{~T}}=1,5 \times 10^{-5} \mathrm{MPa} \\
& \mathrm{A}_{1}=70 \\
& \mathrm{~B}_{1}=22110 \mathrm{MPa}^{-1}
\end{aligned}
$$

No caso de esforço uniaxial de tração, da figura 4.1a pode-se observar que a variável $\mathrm{Y}_{01}$ tem influência sobre o início do trecho não-linear ("softening"). Já a figura $4.1 \mathrm{~b}$ mostra a influência do parâmetro $\mathrm{A}_{1}$ sobre a inclinação inicial do trecho não-linear e no valor da assíntota horizontal. Com relação ao parâmetro $\mathrm{B}_{1}$, a figura 4.1c ilustra sua influência sobre a curvatura do trecho não-linear. Esse último parâmetro possui a característica de variar o valor da deformação correspondente à tensão de pico e possui marcante influência na obtenção da tensão de pico quando comparado com os outros parâmetros. Observa-se das figuras 4.1 que a danificação que ocorre antes da tensão de pico já tem uma influência significativa no regime prépico, deixando a resposta de ser linear um pouco antes daquela tensão. Isso vem ao encontro dos experimentos relatados por TERRIEN (1980) e mencionados no 
capítulo 1 desta tese. Vale lembrar que esse aspecto não é levado em conta em alguns modelos contidos na literatura.

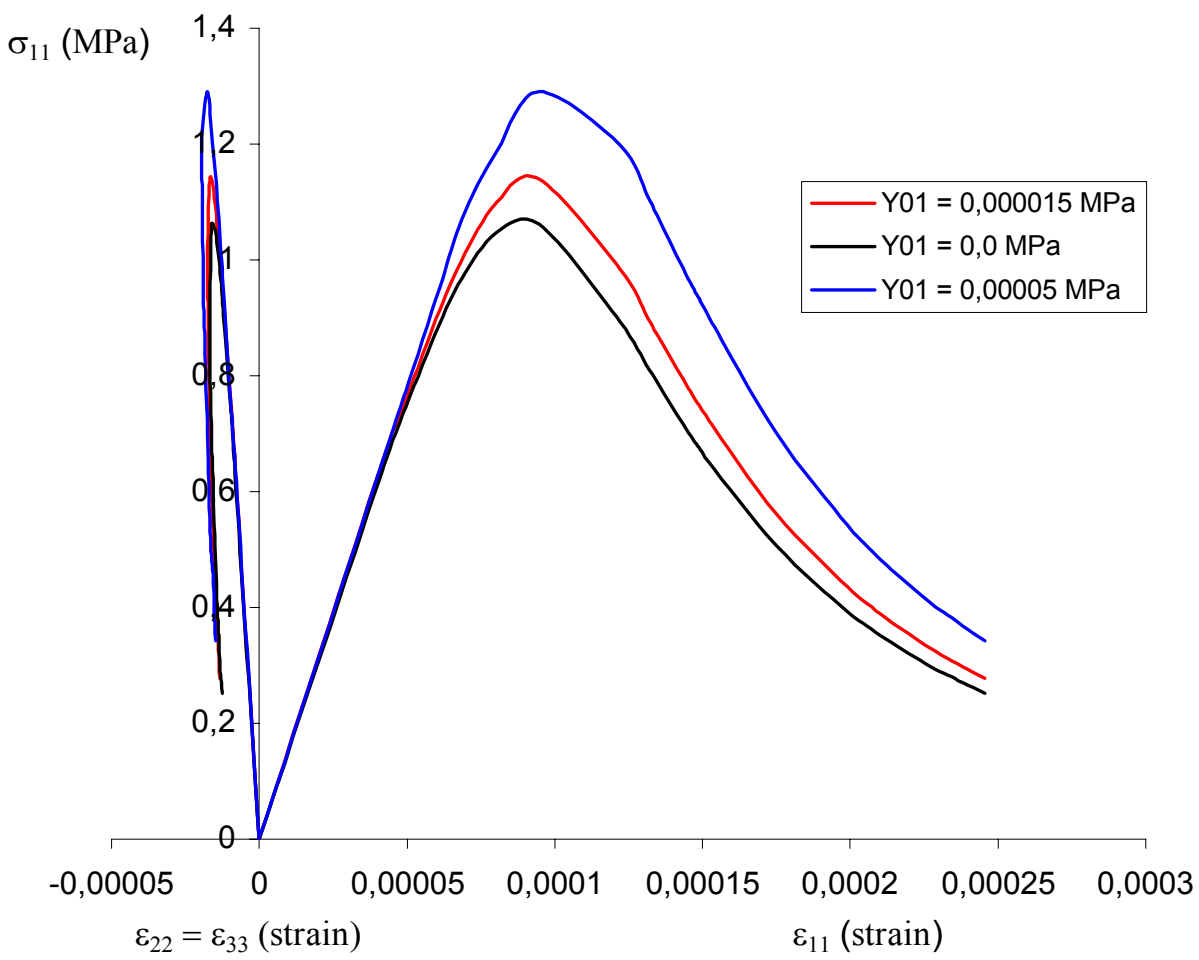

Figura 4.1a - Influência de $Y_{0 T}$ sobre o comportamento em tração

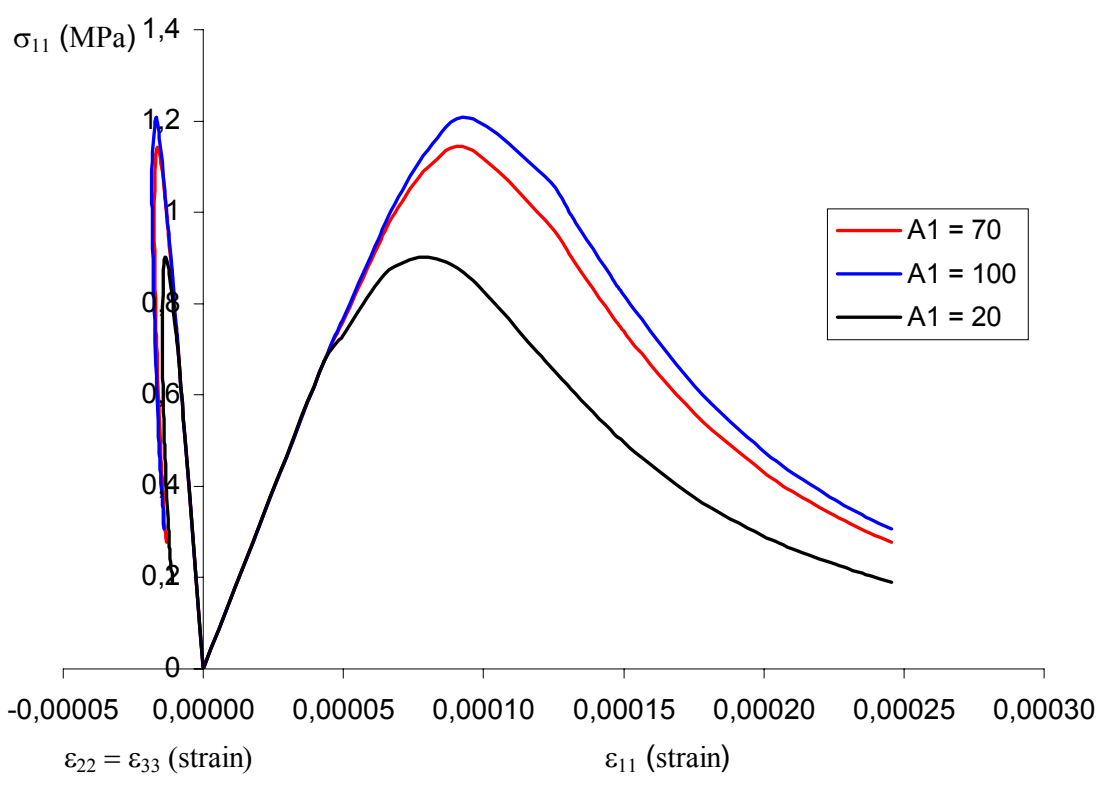

Figura 4.1b - Influência de $A_{1}$ sobre o comportamento em tração 


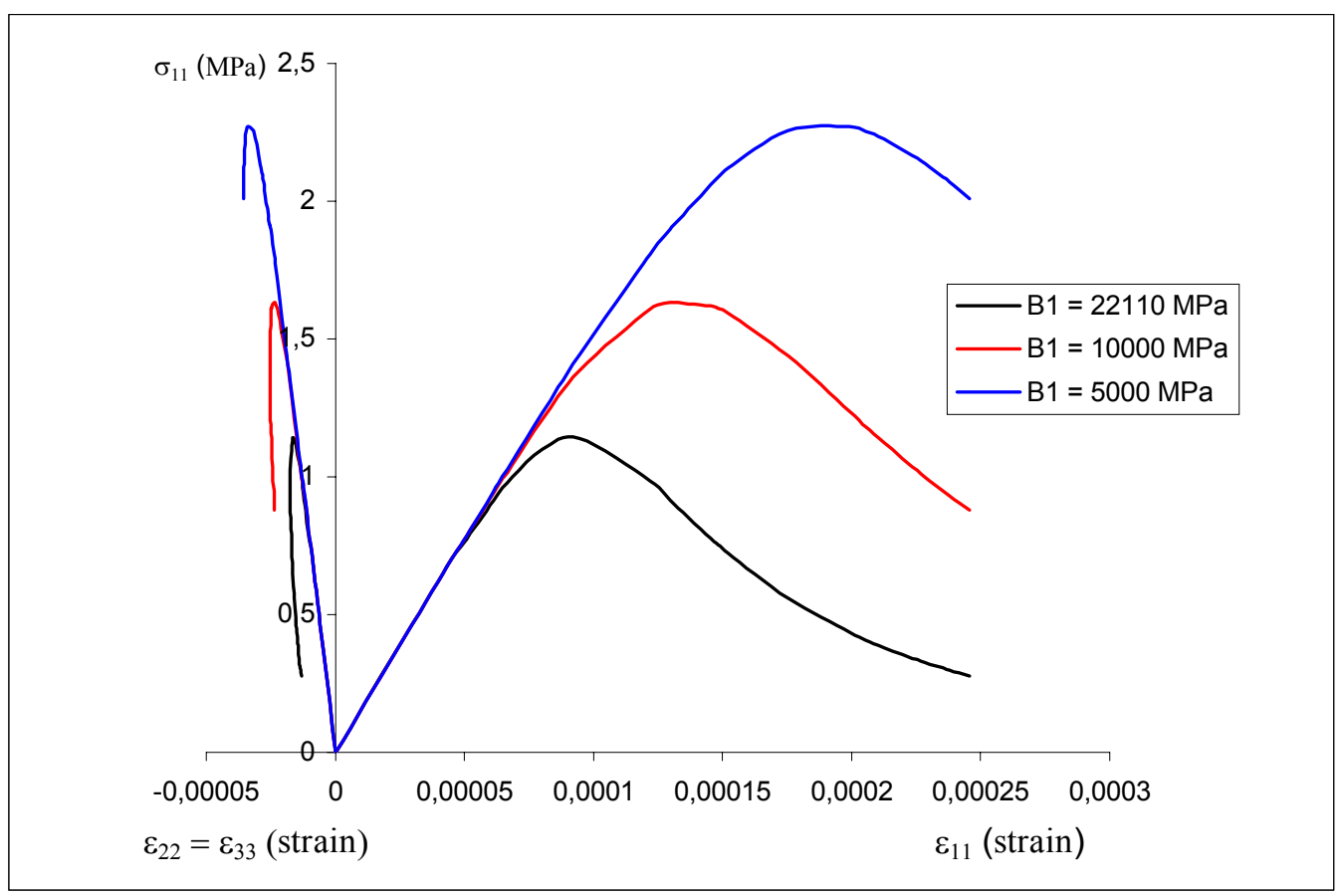

Figura 4.1c - Influência de $B_{1}$ sobre o comportamento em tração

Com relação aos parâmetros de danificação em compressão $\mathrm{Y}_{02}=\mathrm{Y}_{0 \mathrm{C}}, \mathrm{A}_{2} \mathrm{e}$ $\mathrm{B}_{2}$, as figuras $4.2 \mathrm{a}, \mathrm{b}$ e c ilustram a influência de cada um deles na resposta tensãodeformação. Os ensaios numéricos de compressão uniaxial foram realizados variando cada parâmetro a partir dos seguintes valores:

$$
\begin{aligned}
& \mathrm{E}_{0}=31850 \mathrm{MPa} \\
& \mathrm{Y}_{02}=\mathrm{Y}_{0 \mathrm{C}}=2,0 \times 10^{-3} \mathrm{MPa} \\
& \mathrm{A}_{2}=-0,50 \\
& \mathrm{~B}_{2}=5,0 \mathrm{MPa}^{-1}
\end{aligned}
$$

As influências são análogas àquelas observadas nos parâmetros de tração. Observa-se em particular que os parâmetros de compressão $\mathrm{A}_{2}$ e $\mathrm{B}_{2}$ passam a assumir igualdade de importância para a obtenção da tensão de pico. A forma das curvas é quase a mesma quando apenas um dos dois parâmetros varia. Para o parâmetro $\mathrm{Y}_{02}$ valem as mesmas observações feitas para $\mathrm{Y}_{01}$. 


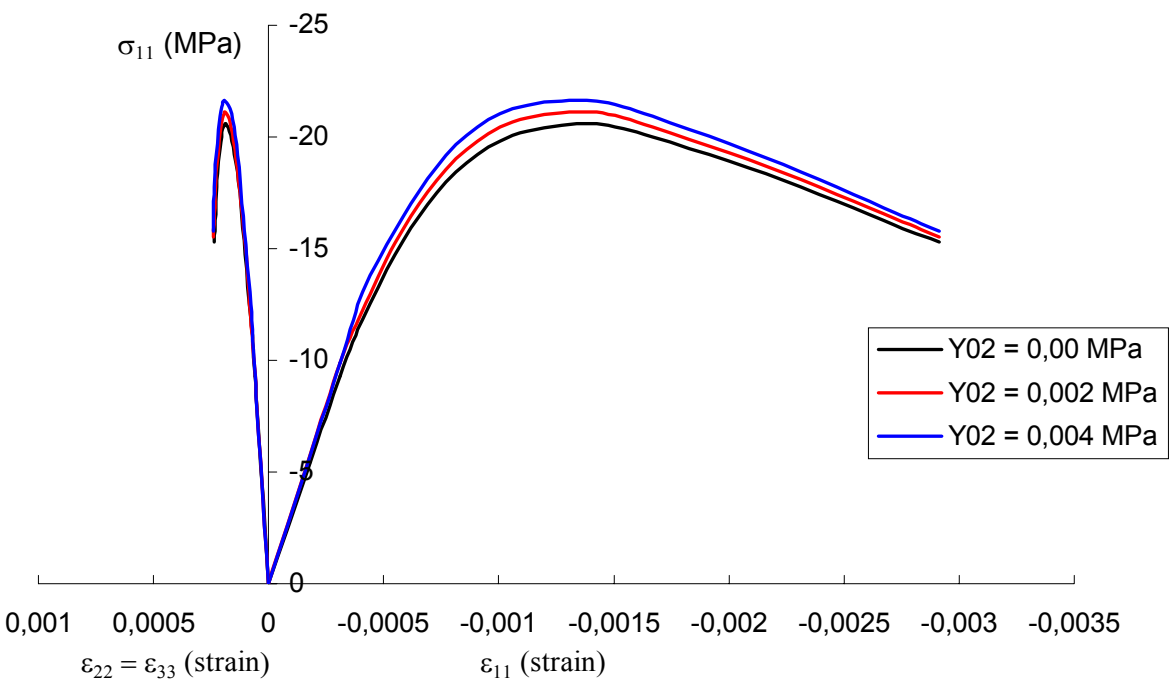

Figura 4.2a - Influência de $Y_{0 C}$ sobre o comportamento em compressão

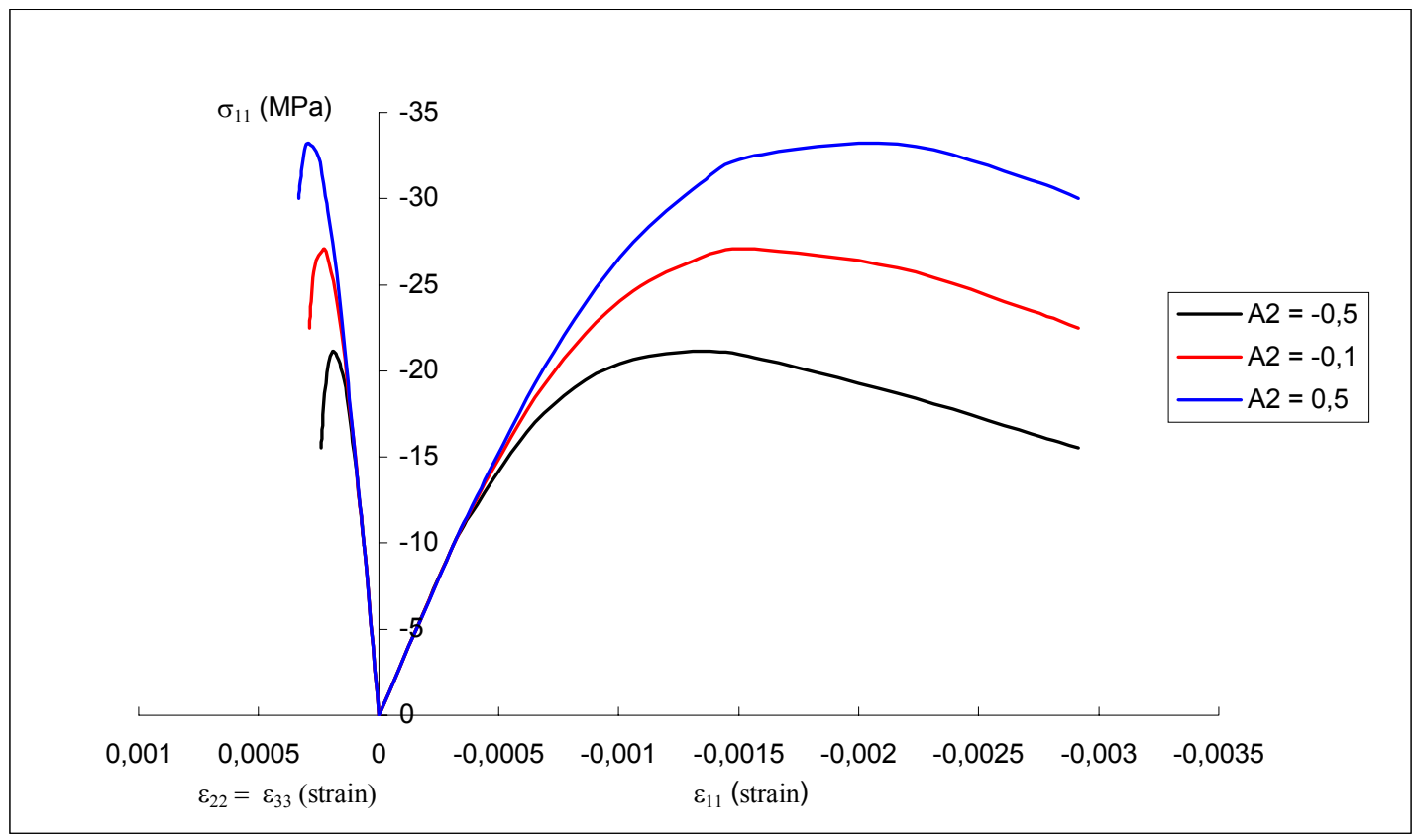

Figura 4.2b - Influência de $A_{2}$ sobre o comportamento em compressão 


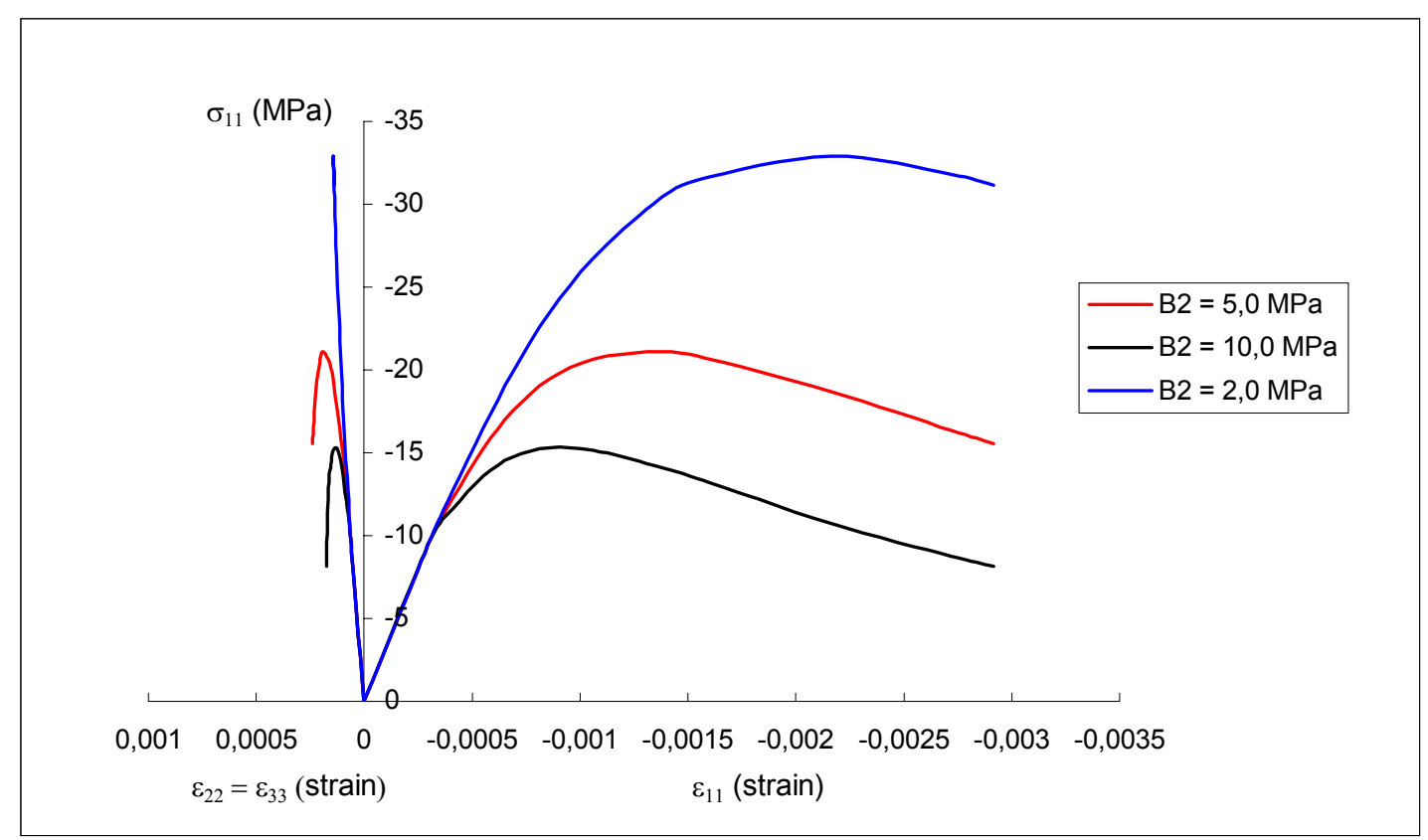

Figura 4.2c - Influência de $B_{2}$ sobre o comportamento em compressão

Com relação aos parâmetros de danificação em compressão $\mathrm{Y}_{03}=\mathrm{Y}_{0 \mathrm{C}}, \mathrm{A}_{3} \mathrm{e}$ $\mathrm{B}_{3}$, as figuras $4.3 \mathrm{a}, \mathrm{b}$ e c ilustram a influência de cada um deles na resposta tensãodeformação. Os ensaios numéricos de compressão biaxial $\left(\sigma_{11}=\sigma_{22}\right)$ foram realizados variando-se cada parâmetro a partir dos seguintes valores:

$\mathrm{E}_{0}=31850 \mathrm{MPa}$

$\mathrm{Y}_{03}=\mathrm{Y}_{0 \mathrm{C}}=2,0 \times 10^{-3} \mathrm{MPa}$

$\mathrm{A}_{3}=-0,60$

$\mathrm{B}_{3}=1,3 \mathrm{MPa}^{-1}$ 


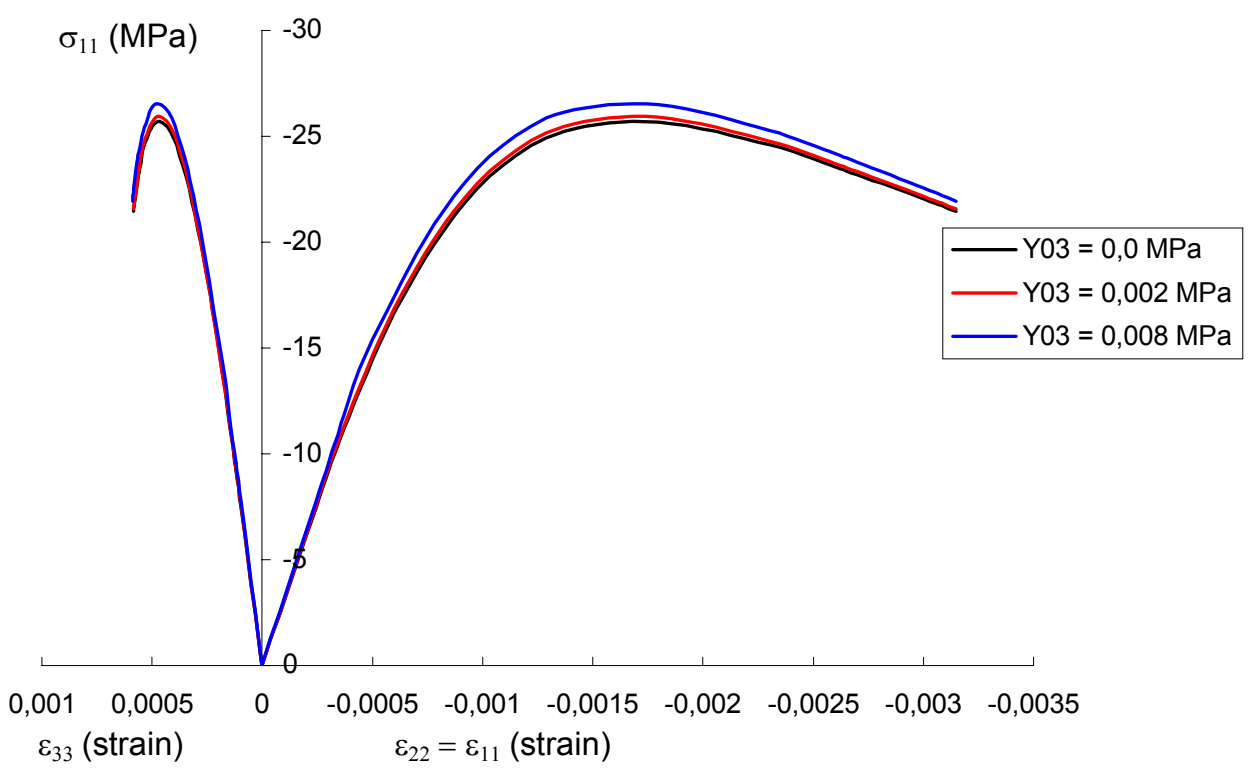

Figura 4.3a - Influência de $Y_{0 \mathrm{C}}$ sobre o comportamento em compressão biaxial

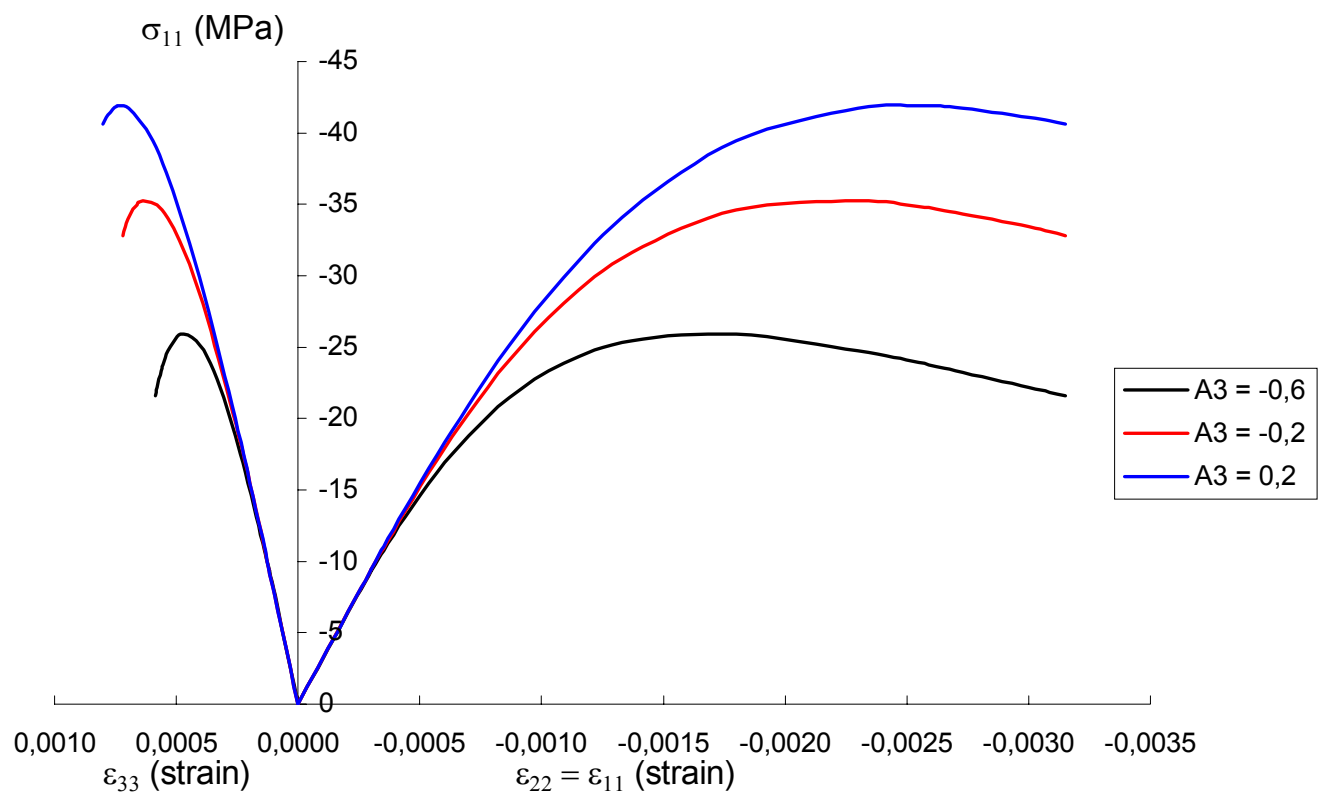

Figura 4.3b - Influência de $A_{3}$ sobre o comportamento em compressão biaxial 


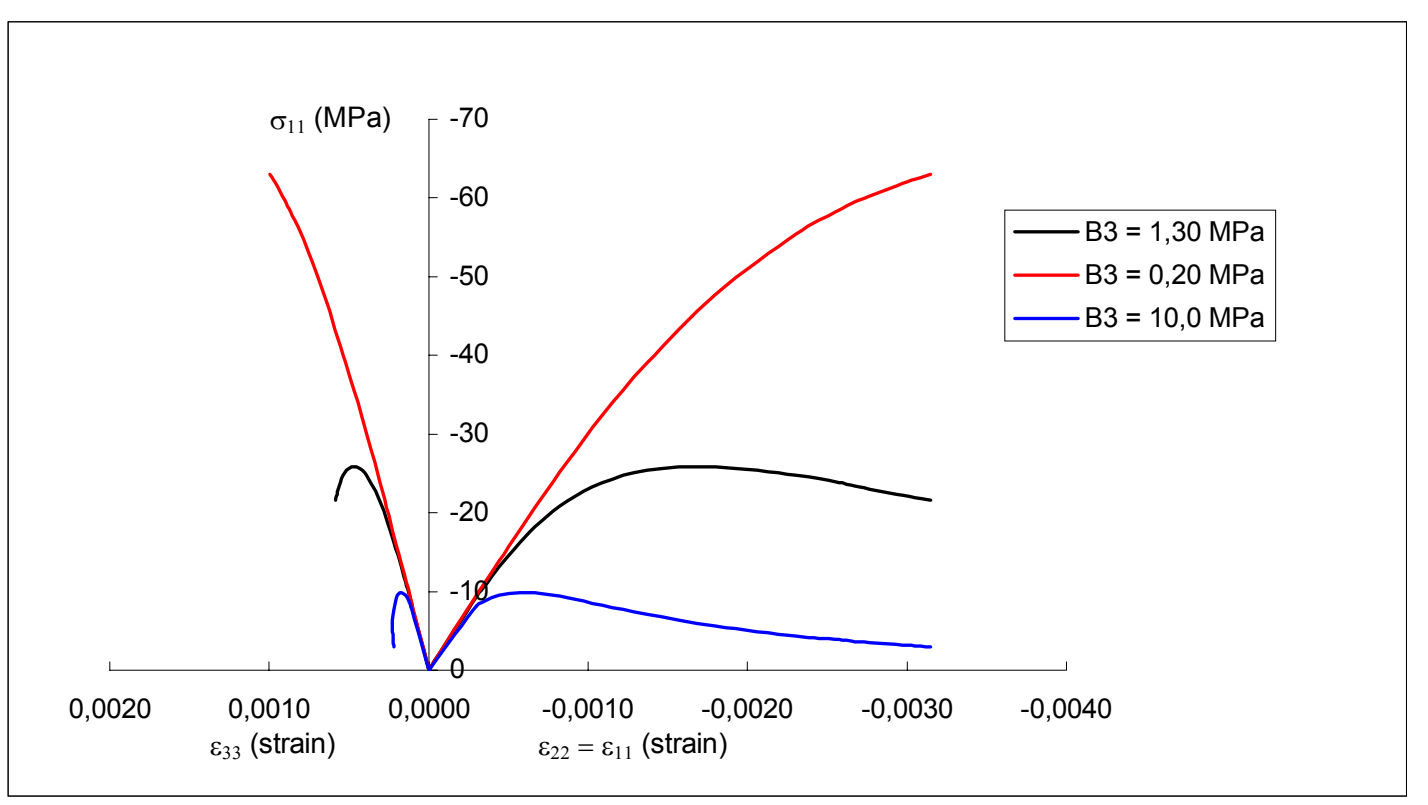

Figura 4.3c - Influência de $B_{3}$ sobre o comportamento em compressão biaxial

Finalmente, nas figuras $4.4 \mathrm{a}$ e $\mathrm{b}$, ilustra-se respectivamente, em ensaios uniaxiais de tração e de compressão, a influência dos parâmetros $\beta_{1}$ e $\beta_{2}$. Os parâmetros anelásticos afetam significativamente a forma do ramo 'softening' no comportamento pós-pico em decorrência das deformações permanentes.

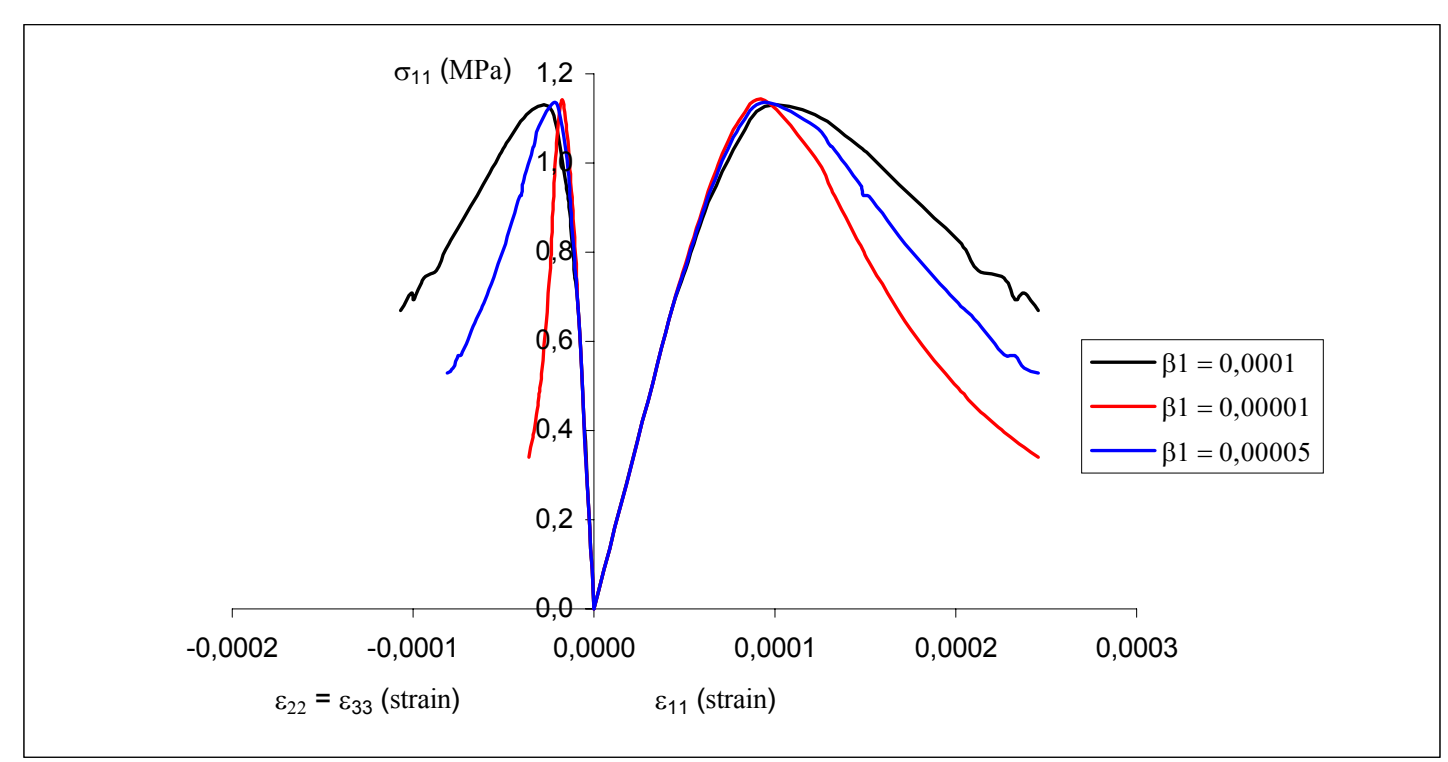

Figura 4.4a - Influência de $\beta_{1}$ sobre o comportamento em tração 


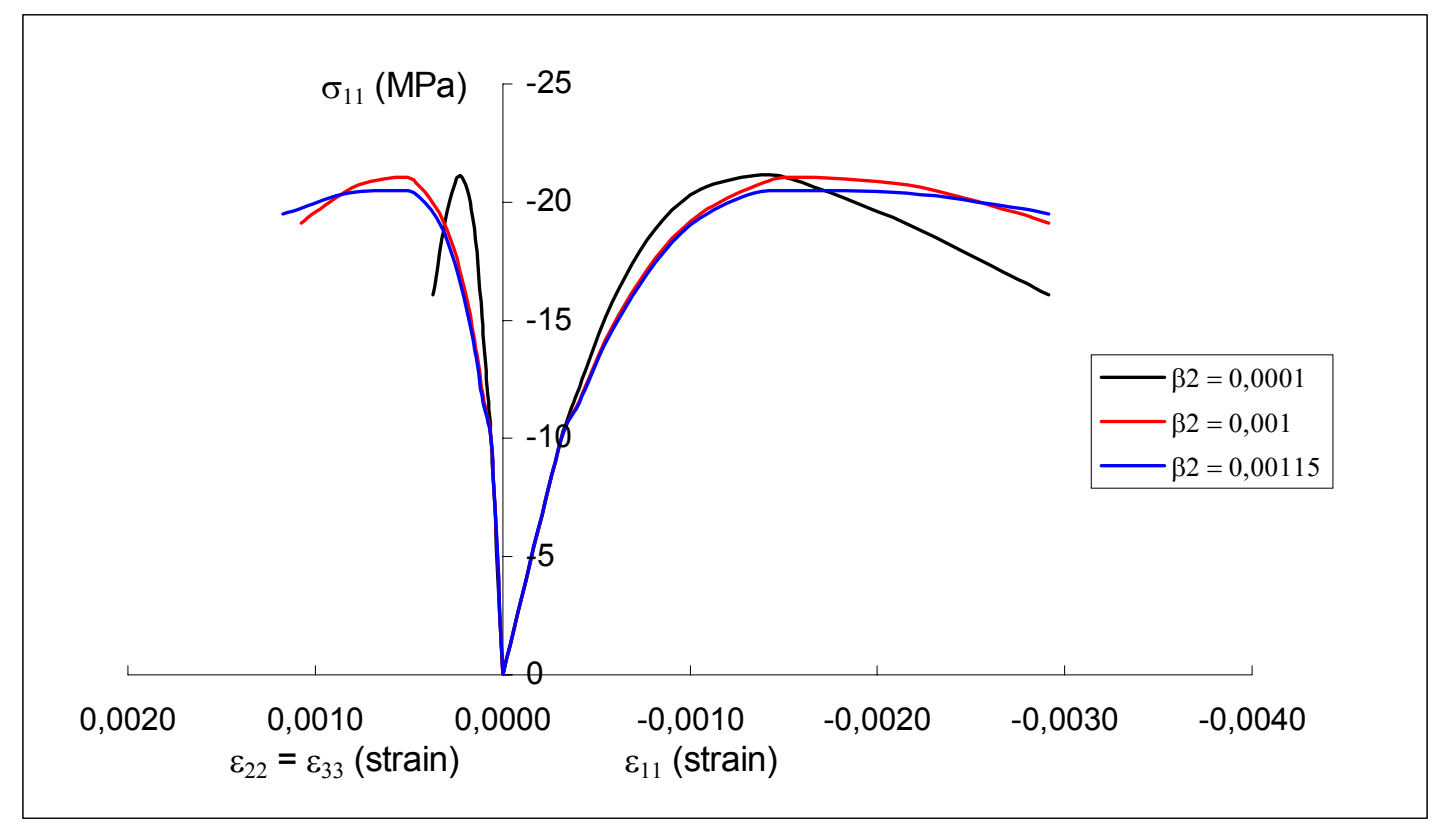

Figura 4.4b - Influência de $\beta_{2}$ sobre o comportamento em compressão

\subsection{RESULTADOS NUMÉRICOS DA CALIBRAÇÃO}

\subsubsection{Modelo de Dano Proposto (sem deformações permanentes)}

O primeiro exemplo trata de um ensaio uniaxial de um corpo de prova de concreto $\left(\mathrm{E}_{0}=15600 \mathrm{MPa}\right.$ e $\left.v_{0}=0.2\right)$ submetido a uma força de tração. Os resultados experimentais foram apresentados por MAZARS, BERTHAUD \& RAMTANI (1990) num ensaio denominado PIED ("Pour Identifier L'Endommagement Diffus"). Os parâmetros do modelo foram obtidos por calibração da curva tensão normal contra deformação na direção da força aplicada, resultando em:

$\mathrm{A}_{1}=70 ; \mathrm{B}_{1}=22110 \mathrm{MPa}^{-1} ; \mathrm{Y}_{01}=1.5 \times 10^{-5} \mathrm{MPa}$

$\mathrm{Na}$ figura 4.5 a resposta numérica fornecida pelo modelo proposto é comparada com a resposta experimental do ensaio PIED. Observa-se que as deformações transversais ilustradas (direções 2 e 3) correspondem apenas aos 
resultados obtidos com os parâmetros identificados, não havendo registro experimental para confronto.

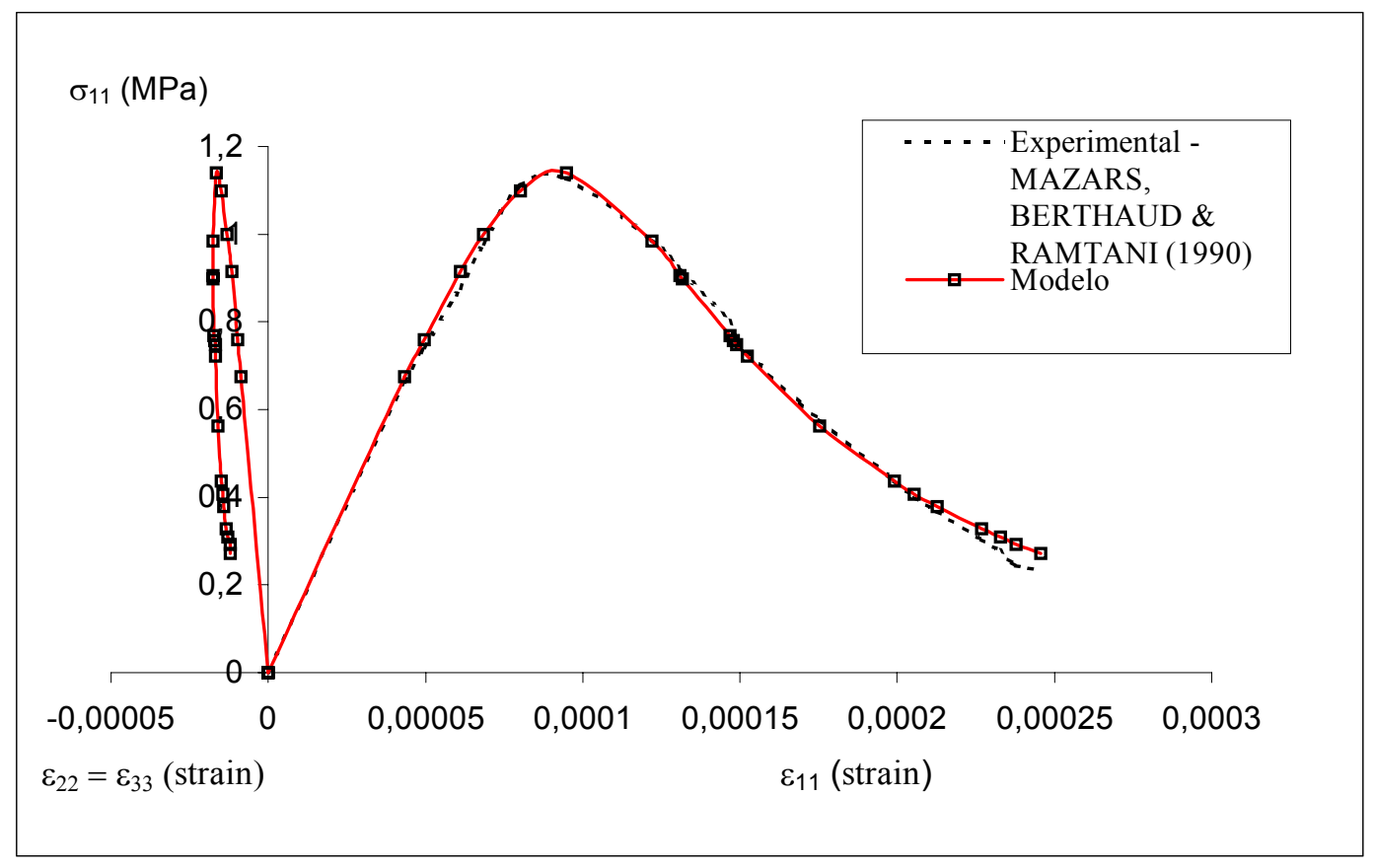

Figura 4.5 - Simulação do teste de tração uniaxial

[MAZARS, BERTHAUD \& RAMTANI (1990)].

Pelos resultados, nota-se que o conjunto de parâmetros identificados para o modelo permite reproduzir bastante bem os dados experimentais. Quanto às deformações transversais previstas pelo modelo, um bom indicativo é que elas estão em correspondência com a forma descrita em WILLAM, STANKOWSKI \& STURE (1988).

O modelo também foi empregado na simulação de ensaios biaxiais e uniaxiais de tensão realizados por KUPFER, HILSDORF \& RUSH (1969) em espécimes de concreto. O módulo de elasticidade e o coeficiente de Poisson utilizados na simulação numérica foram: $\mathrm{E}_{0}=31850 \mathrm{MPa}, v_{0}=0.2$.

Chama-se a atenção para o fato de que em casos mais complexos de tensão, a calibração do conjunto de parâmetros que aparecem nas leis de evolução das variáveis de dano envolvidas exige ensaios uniaxiais e biaxiais. Em particular, os parâmetros relativos às variáveis $\mathrm{D}_{1}$ e $\mathrm{D}_{2}$ foram determinados por ensaios uniaxiais de tração e de compressão, respectivamente: 


$$
\begin{aligned}
& \mathrm{A}_{1}=69.4 ; \mathrm{B}_{1}=9500 \mathrm{MPa}^{-1} ; \mathrm{Y}_{01}=0.8 \times 10^{-4} \mathrm{MPa} \\
& \mathrm{A}_{2}=-0.80 ; \mathrm{B}_{2}=0.90 \mathrm{MPa}^{-1} ; \mathrm{Y}_{02}=0.2 \times 10^{-2} \mathrm{MPa}
\end{aligned}
$$

Já os parâmetros relativos a $\mathrm{D}_{3}$ foram obtidos do ensaio de compressão biaxial $\left(\sigma_{11}=\sigma_{22}\right)$, através da calibração das curvas experimentais tensãodeformação nas direções 1 e 2. Os parâmetros obtidos foram:

$$
\mathrm{A}_{3}=-0.60 ; \mathrm{B}_{3}=1.305 \mathrm{MPa}^{-1} ; \mathrm{Y}_{03}=\mathrm{Y}_{02}=0.2 \times 10^{-2} \mathrm{MPa}
$$

A figura 4.6 mostra a comparação entre as curvas experimentais tensãodeformação e as curvas obtidas com o modelo proposto para testes de compressão uniaxial e biaxial com diferentes níveis de solicitação.

Vale ressaltar que as outras combinações de solicitação, por exemplo, compressão biaxial $\sigma_{22}=0.52 \sigma_{11}$, compressão-tração, foram simuladas com os parâmetros $A_{i}, B_{i}$ e $Y_{0 i}$ acima descritos.

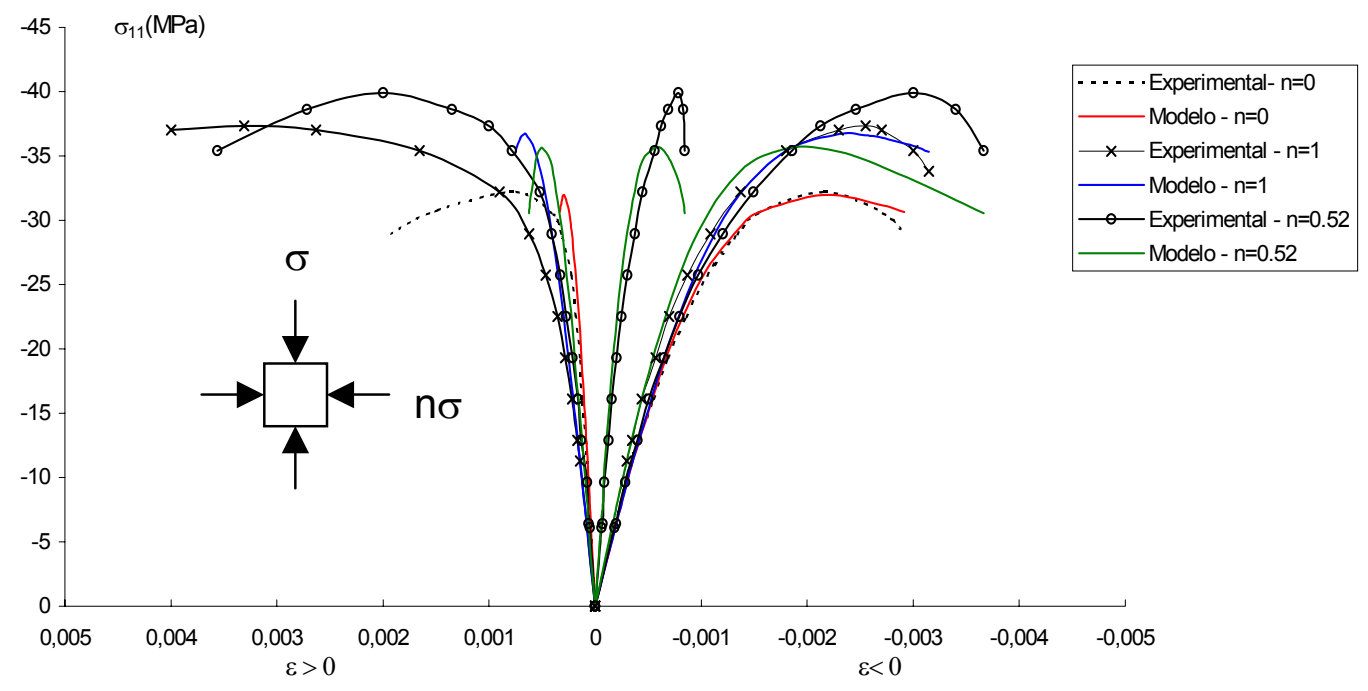

Figura 4.6 - Simulação de testes uniaxiais e biaxiais [KUPFER, HILSDORF \& RUSH (1969)]: Resultados experimentais e numéricos

Observa-se na figura acima uma boa concordância entre resultados experimentais e numéricos nas curvas que envolvem as deformações diretas, de 
compressão nos casos analisados. Porém, os resultados das deformações transversais, ou de tração, subestimam a ductilidade observada experimentalmente. Isso se deve ao fato que na região próxima à tensão de pico, as deformações residuais passam a desempenhar um papel importante no comportamento do concreto. Como o modelo despreza tais deformações, sua resposta não condiz com a realidade experimental em tais situações. Essas mesmas considerações são válidas para a análise das deformações volumétricas ilustradas na figura 4.7. Como o modelo proposto não leva em conta deformações residuais, não é possível prever a mudança de sinal da deformação volumétrica observada experimentalmente particularmente no ensaio de compressão uniaxial, levando a uma discrepância dos valores obtidos com o modelo quando comparados aos valores experimentais na região próxima à tensão de pico.

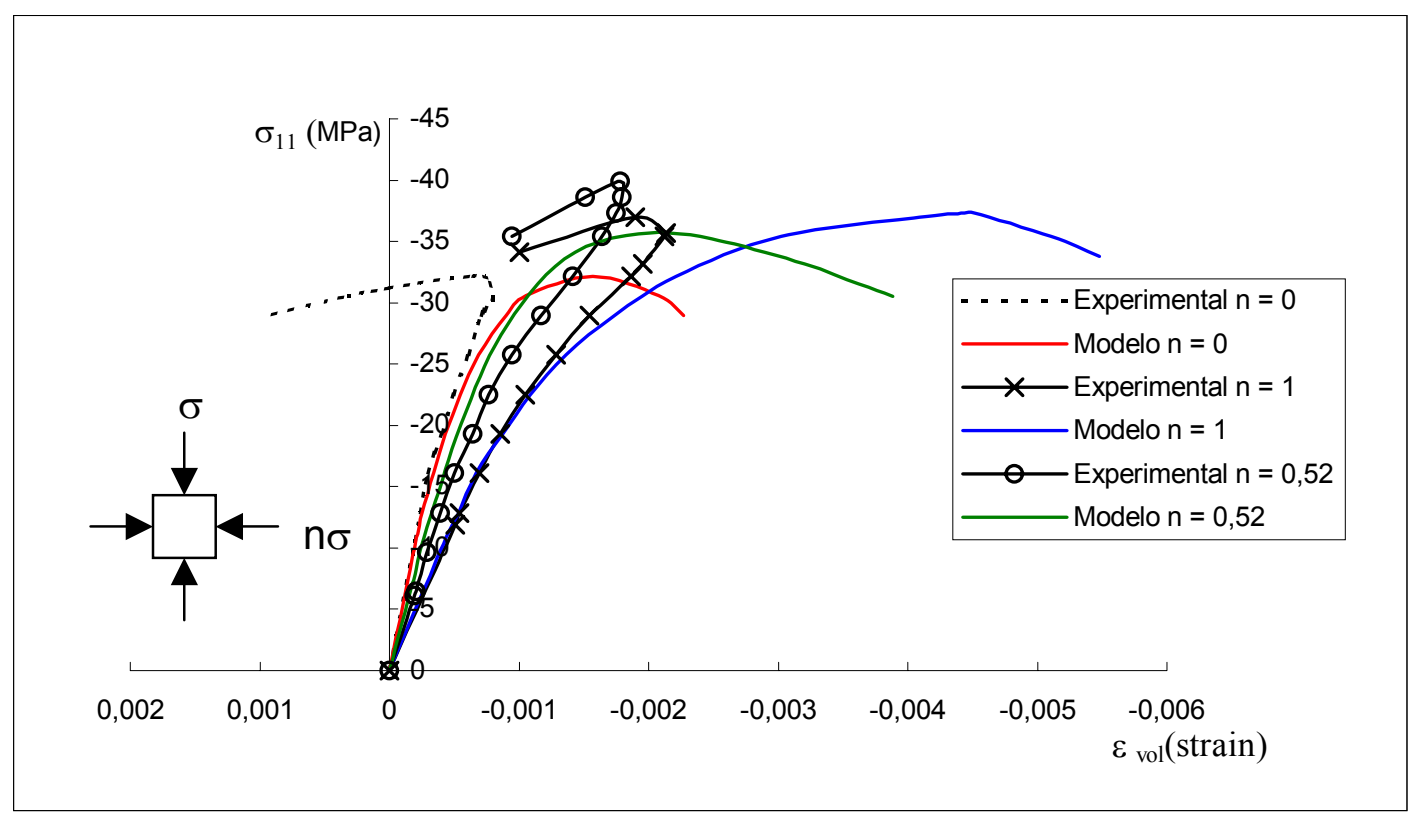

Figura 4.7 - Curvas de tensão $x$ deformação volumétrica em compressão uniaxial e biaxial [KUPFER, HILSDORF \& RUSH (1969)]:

Resultados experimentais e numéricos

Um outro resultado de interesse é o domínio de ruptura, caracterizado pelos picos de tensão, obtido numericamente em correspondência a solicitações uniaxiais e biaxiais. A resposta obtida está representada na figura 4.8, juntamente com os resultados experimentais. Pode-se observar que o modelo consegue prever o domínio de ruptura satisfatoriamente. 


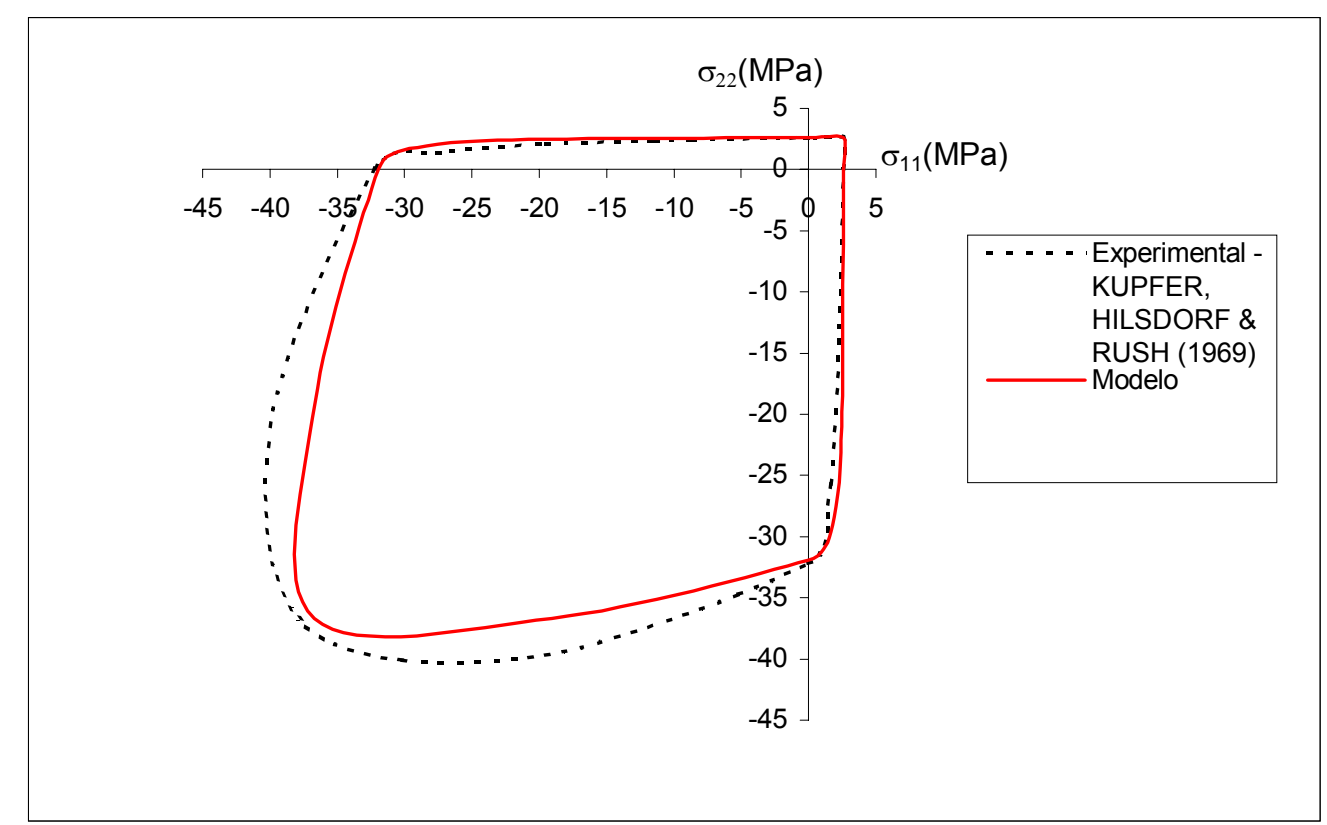

Figura 4.8 - Simulação de testes uniaxiais e biaxiais [KUPFER, HILSDORF \& RUSH (1969)]: Resultados experimentais e numéricos.

Por fim, considera-se a aplicação do modelo na simulação numérica de um ensaio realizado por VAN MIER (1984), em corpo de prova de concreto $\left(\mathrm{E}_{0}=23250\right.$ MPa e $\left.v_{0}=0.2\right)$ submetido a uma compressão triaxial segundo a relação $\sigma_{11}<0 ; \sigma_{22}$ $=0.10 \sigma_{11} ; \sigma_{33}=0.05 \sigma_{11}$.

Como a resposta experimental em compressão uniaxial e biaxial deste concreto não é conhecida, optou-se por fazer a calibração dos parâmetros do modelo através da curva experimental tensão-deformação na direção 1. A obtenção dos parâmetros relativos às variáveis de dano $\mathrm{D}_{2}$ e $\mathrm{D}_{3}$ resulta em:

$$
\begin{aligned}
& \mathrm{A}_{2}=-0.80 ; \mathrm{B}_{2}=0.15 \mathrm{MPa}^{-1} \\
& \mathrm{~A}_{3}=-0.60 ; \mathrm{B}_{3}=1.305 \mathrm{MPa}^{-1} ; \mathrm{Y}_{02}=\mathrm{Y}_{03}=0.2 \times 10^{-2} \mathrm{MPa}
\end{aligned}
$$

A figura 4.9 ilustra a comparação entre os resultados fornecidos pelo modelo e os resultados experimentais obtidos por VAN MIER (1984). 


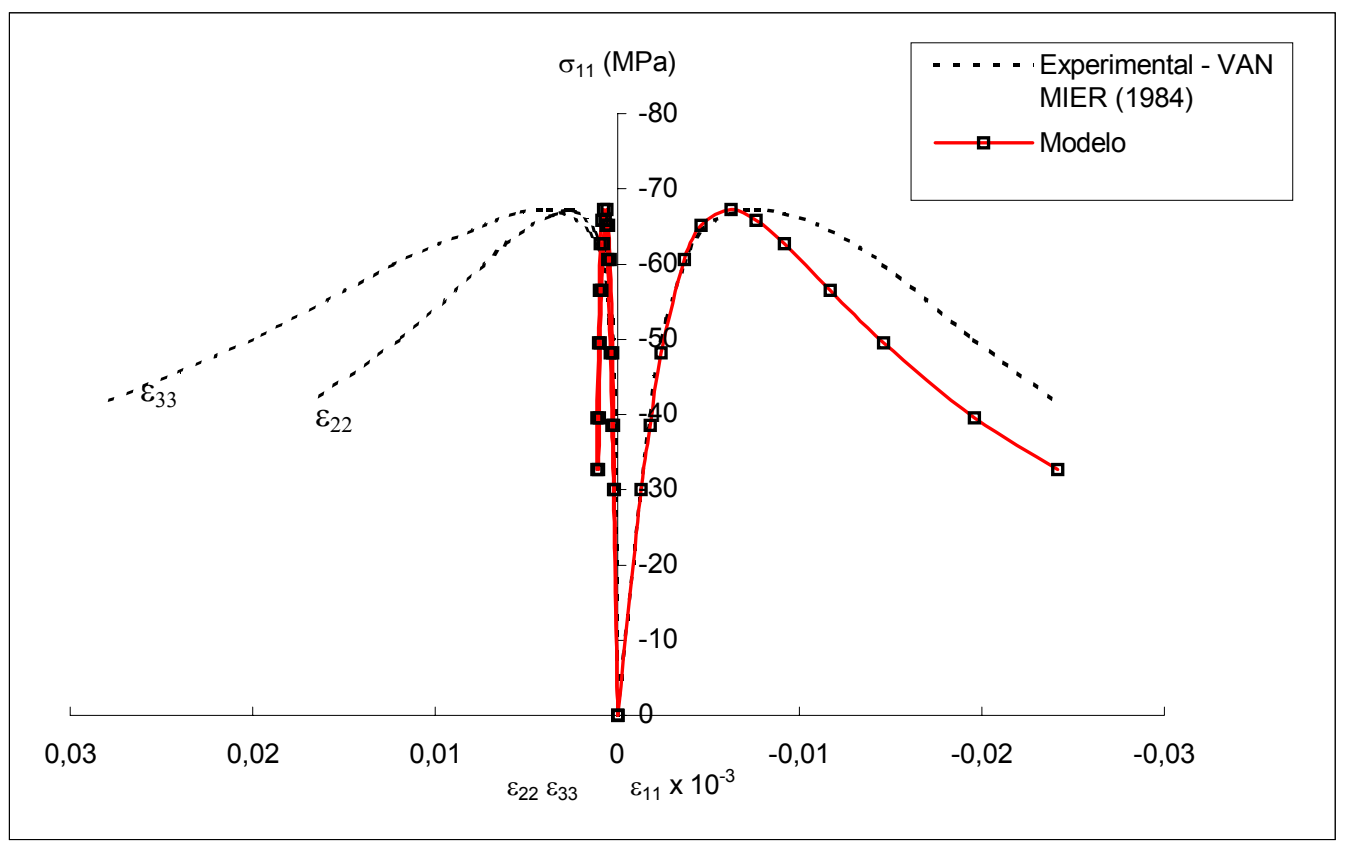

Figura 4.9 - Simulação de teste de compressão triaxial [VAN MIER (1984)]:

Resultados experimentais e numéricos

Observa-se na curva $\sigma_{11}$ x $\varepsilon_{11}$ uma boa concordância entre os resultados experimentais e numéricos. Porém, os resultados das deformações transversais, de tração neste caso, não conseguem reproduzir a evidente ductilidade experimental. Mais uma vez a desconsideração de deformações residuais aparece com forte influência no comportamento pós-pico. Contudo, o modelo é capaz de simular de modo bastante razoável o comportamento experimental até a tensão máxima.

De uma maneira geral, os resultados da calibração do modelo foram satisfatórios, principalmente no que diz respeito à recuperação dos valores de tensão de pico e da resposta constitutiva do concreto até aqueles valores de tensão. Destacam-se, além disso, as simulações bastante precisas das curvas tensãodeformação em tração e compressão uniaxial e a obtenção da envoltória de ruptura do concreto em estados biaxiais de tensão.

Vale destacar, também, certos aspectos evidenciados pelo modelo e que são consistentes com evidências experimentais observadas no concreto, tais como:

- A forma da curva tensão-deformação uniaxial em tração, que apresenta um 'softening' pronunciado;

- o acréscimo da tensão de pico comparando-se a compressão uniaxial com a biaxial; 
A potencial capacidade do modelo de simular o comportamento unilateral do concreto não foi explorada pelos exemplos, mas em tese o modelo permite que se façam simulações envolvendo inversões de sinais das solicitações.

O fato de, nesta etapa, não terem sido levadas em conta as deformações residuais não implica que o modelo, com essa limitação, não seja de serventia para a análise de estruturas de concreto, como se verá no próximo capítulo.

Os resultados obtidos apontam, ainda, que as deformações residuais podem ter significativa importância nas respostas pós-pico. Para confirmar essa afirmação, serão apresentadas respostas numéricas que consideram deformações permanentes na formulação do modelo proposto originalmente (ver capítulo 3).

\subsubsection{Emprego da Versão Uniaxial do Modelo de Dano Proposto Considerando-se Deformações Anelásticas}

Este item tem por objetivo apresentar a potencialidade do modelo com a consideração de deformações plásticas.

O modelo foi empregado na simulação do ensaio uniaxial de compressão realizado por KUPFER, HILSDORF \& RUSH (1969) em espécimes de concreto. O módulo de elasticidade e o coeficiente de Poisson utilizados na simulação numérica permanecem os mesmos: $\mathrm{E}_{0}=31850 \mathrm{MPa}, v_{0}=0.2$.

Os parâmetros do modelo foram obtidos pela calibração da curva tensãodeformação experimental, resultando em:

$\mathrm{A}_{2}=0,70 ; \mathrm{B}_{2}=5,50 \mathrm{MPa}^{-1} ; \mathrm{Y}_{02}=2,0 \times 10^{-3} \mathrm{MPa}, \beta_{2}=1,58 \times 10^{-3}$

A figura 4.10 evidencia que a incorporação das deformações plásticas na formulação do modelo melhora significantemente a captura das deformações transversais $\left(\varepsilon_{22}=\varepsilon_{33}\right)$. 


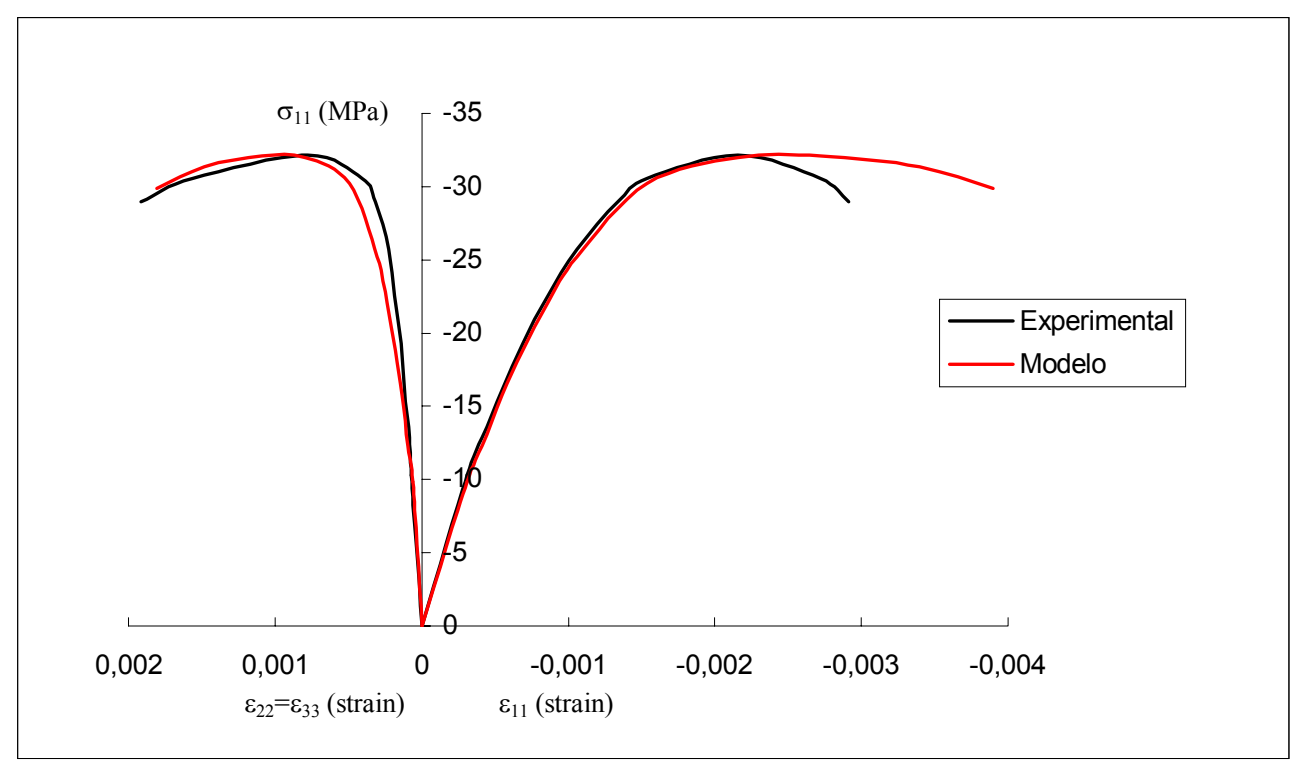

Figura 4.10 - Simulação do teste de compressão uniaxial

[KUPFER, HILSDORF \& RUSH (1969)]:

Resultados experimentais e numéricos

Além disso, o modelo prevê corretamente a inversão no sentido da deformação volumétrica, observada experimentalmente. Na figura 4.11 apresenta-se um confronto entre as respostas com deformações permanentes e com o modelo originalmente proposto.

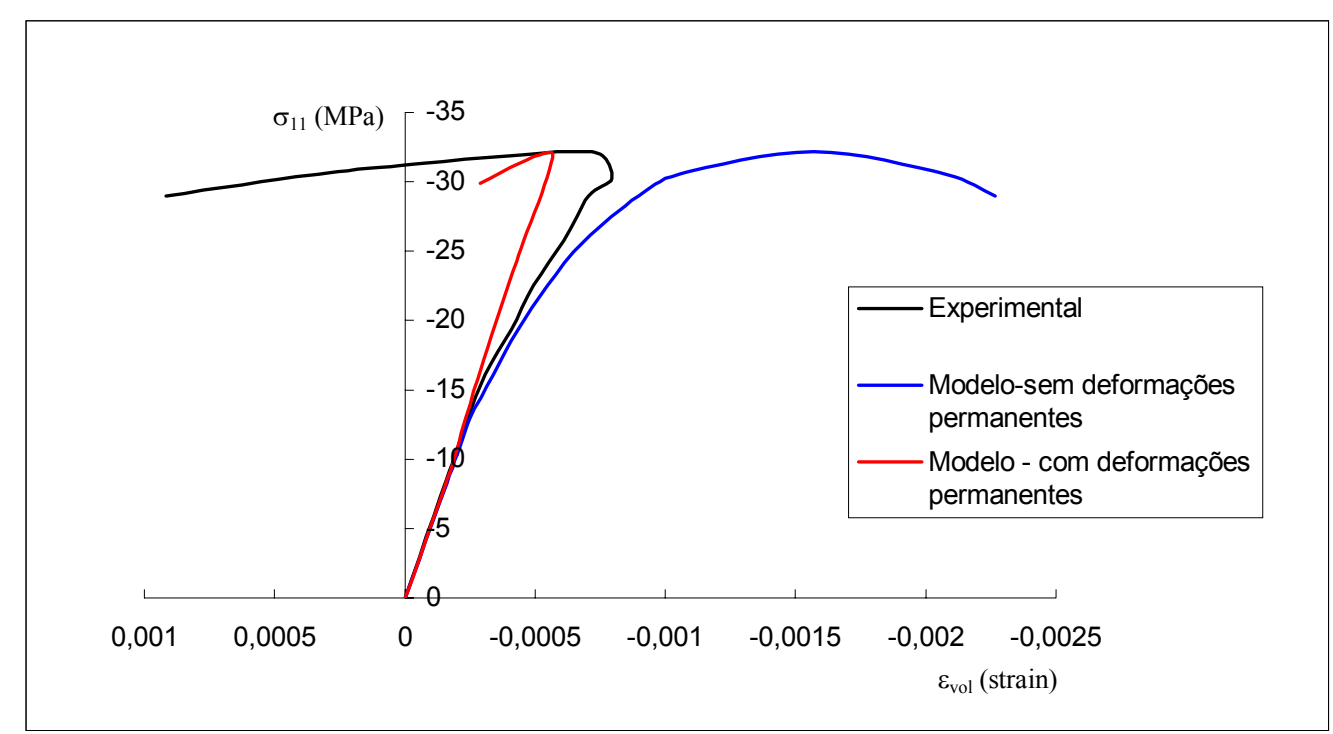

Figura 4.11 - Curvas de tensão x deformação volumétrica em compressão uniaxial [KUPFER, HILSDORF \& RUSH (1969)]:

Resultados experimentais e numéricos 


\section{EXEMPLOS DE APLICAÇÃO DO MODELO PROPOSTO}

\subsection{INTRODUÇÃO}

O presente capítulo tem por objetivo principal avaliar o modelo proposto pela sua aplicação na análise de estruturas em concreto armado. Para este fim são realizadas análises uni e bidimensionais, tendo sido implementado o modelo em códigos de cálculo baseados no Método dos Elementos Finitos. Os resultados obtidos pelo modelo são confrontados com resultados experimentais e com as respostas fornecidas pelos modelos de dano de LA BORDERIE (1991) e MAZARS (1984).

Inicialmente são avaliadas as respostas de simulações que empregam a versão unidimensional do modelo. No âmbito dessa análise, um primeiro exemplo é composto por três vigas em concreto armado, de mesmas dimensões, mas com diferentes taxas de armadura, apresentadas originalmente em ÁLVARES (1993). O confronto entre os resultados numéricos e experimentais é ilustrado sob a forma de curvas carga aplicada por deslocamento vertical no meio do vão das vigas. Um segundo exemplo trata de um pórtico plano em concreto armado [VECCHIO \& EMARA (1992)]. A resposta numérica fornecida pelo modelo é confrontada com a experimental e com as obtidas pelos modelos de LA BORDERIE (1991) e MAZARS (1984).

Com relação às simulações bidimensionais, o modelo proposto é inicialmente empregado na análise de uma viga biapoiada em concreto armado descrita em PEREGO (1989). Confrontam-se as respostas experimentais com as curvas numéricas resultantes da aplicação do modelo proposto e do modelo de MAZARS (1984). Além disso, ilustra-se a configuração de danificação obtida com o modelo, comparando-a com a configuração descrita em PEREGO (1989). Num último caso, 
emprega-se o modelo proposto nas mesmas vigas de ÁLVARES (1993) citadas anteriormente. A análise bidimensional evidenciou, nesse caso, alguns problemas de ordem numérica que são discutidos no final deste capítulo.

\subsection{ANÁLISES UNIDIMENSIONAIS}

As versões unidimensionais dos modelos foram implementadas num programa para análise de estruturas de barras discretizadas com elementos finitos estratificados em camadas (EFICoS - “Eléments Finis à Couches Superposées”).

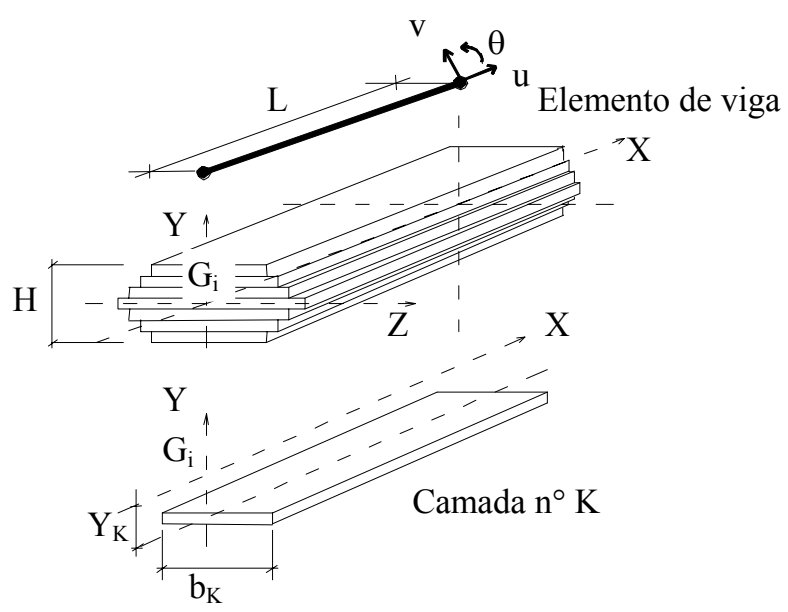

Figura 5.1 - Elemento finito empregado

O elemento finito unidimensional com camadas superpostas é baseado num elemento de pórtico clássico com dois nós, onde cada nó possui três graus de liberdade $\mathrm{u}$, v e $\theta$ (Figura 5.1).

São assumidas, como hipóteses de cálculo, a negligência de deformações por distorção. Para o concreto valem os modelos de danificação em estudo, e para as barras de aço longitudinais admite-se um comportamento elasto-plástico.

Na seção transversal em concreto armado discretizada, uma certa camada pode conter aço e concreto. Admitindo-se perfeita aderência entre os materiais, definem-se, para a camada em questão, um módulo elástico e uma deformação anelástica equivalentes, utilizando-se a seguinte regra de homogeneização: 
$\mathrm{E}_{\mathrm{k}}=\left(1-\mathrm{C}_{\mathrm{ak}}\right) \mathrm{E}_{\mathrm{ck}}+\mathrm{C}_{\mathrm{ak}} \mathrm{E}_{\mathrm{ak}}$

$\varepsilon_{\mathrm{ank}}=\left(1-\mathrm{C}_{\mathrm{ak}}\right) \varepsilon_{\mathrm{ank}}+\mathrm{C}_{\mathrm{ak}} \varepsilon_{\mathrm{pk}}$

onde:

- $\mathrm{C}_{\mathrm{ak}}$ é a concentração volumétrica de aço na camada $\mathrm{n}^{\circ} \mathrm{k}$

- $\quad E_{a k}$ é o módulo de elasticidade do aço na camada $n^{\circ} k$

- $\quad E_{c k}$ é o módulo de elasticidade do concreto na camada $n^{\circ} \mathrm{k}$

- $\quad \varepsilon_{\mathrm{pk}}$ é a deformação plástica do aço na camada $\mathrm{n}^{\circ} \mathrm{k}$

- $\quad \varepsilon_{\text {ankk }}$ é a deformação anelástica na camada ${ }^{\circ} \mathrm{k}$

- $\quad E_{k}$ é o módulo de elasticidade equivalente da camada $n^{\circ} k$

- $\quad \varepsilon_{\text {ank }}$ é a deformação anelástica do concreto na camada $\mathrm{n}^{\circ} \mathrm{k}$ [caso do modelo de LA BORDERIE (1991)]

Detalhes sobre a formulação dos modelos de dano de MAZARS (1984) e de LA BORDERIE (1991) podem ser encontrados em PITUBA (1998).

\subsubsection{Vigas em Concreto Armado [ÁLVARES (1993)]}

As vigas em questão são biapoiadas, com 2,40 m de vão, seção transversal retangular $(12 \times 30 \mathrm{~cm})$ e o carregamento é constituído por duas forças concentradas iguais aplicadas nos terços dos vãos. Na confecção das vigas foi utilizado um concreto com $\mathrm{E}_{\mathrm{c}}=29200 \mathrm{MPa}$. Para o aço das armaduras adotou-se $\mathrm{E}_{\mathrm{a}}=196000$ MPa e tensão de escoamento de $420 \mathrm{MPa}$, admitindo-se um comportamento elastoplástico perfeito. O coeficiente de Poisson adotado foi de 0,20. Maiores detalhes sobre a resposta experimental de cada tipo de viga, colhida a partir de provas realizadas com controle de carga, encontram-se em ÁLVARES (1993). Na figura 5.2 são fornecidos os detalhes de geometria e armação das vigas. 


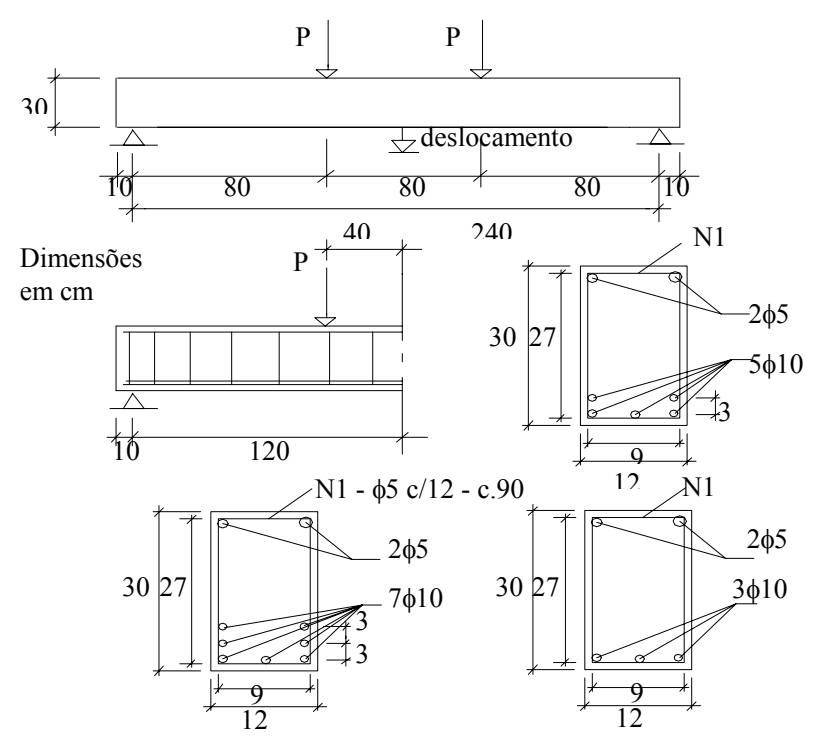

Figura 5.2 - Geometria e armação das vigas

Os parâmetros de compressão de todos os modelos empregados na análise numérica foram identificados a partir das medidas de ensaios experimentais em corpos de prova submetidos à compressão uniaxial, realizados por ÁLVARES (1993). Já os parâmetros de tração foram identificados sobre uma resposta em tração uniaxial descrita pelo modelo de Mazars e proposta por ÁLVARES (1993) para o concreto das vigas.

$\mathrm{Na}$ tabela a seguir são fornecidos os valores dos parâmetros utilizados em cada modelo. As figuras 5.3 e 5.4 representam as respostas numéricas de cada um dos modelos nos casos uniaxiais.

Tabela 5.1 - Parâmetros dos modelos de Mazars, de La Borderie e do proposto.

\begin{tabular}{|c|l|l|l|l|}
\hline \multirow{2}{*}{ Mazars } & \multicolumn{2}{|c|}{ La Borderie } & \multicolumn{2}{c|}{ Proposto } \\
\cline { 2 - 5 } & $\begin{array}{c}\text { Parâmetros } \\
\text { de Tração }\end{array}$ & $\begin{array}{c}\text { Parâmetros } \\
\text { de Compressão }\end{array}$ & $\begin{array}{c}\text { Parâmetros } \\
\text { de Tração }\end{array}$ & $\begin{array}{c}\text { Parâmetros de } \\
\text { Compressão }\end{array}$ \\
\hline $\mathrm{A}_{\mathrm{T}}=0,995$ & $\mathrm{Y}_{01}=3,05 \times 10^{-4} \mathrm{MPa}$ & $\mathrm{Y}_{02}=0,5 \times 10^{-2} \mathrm{MPa}$ & $\mathrm{Y}_{01}=0,72 \times 10^{-4} \mathrm{MPa}$ & $\mathrm{Y}_{02}=0,5 \times 10^{-3} \mathrm{MPa}$ \\
\hline $\mathrm{B}_{\mathrm{T}}=8000$ & $\mathrm{~A}_{1}=3,50 \times 10^{+3} \mathrm{MPa}^{-1}$ & $\mathrm{~A}_{2}=6,80 \mathrm{MPa}^{-1}$ & $\mathrm{~A}_{1}=50$ & $\mathrm{~A}_{2}=-0,9$ \\
\hline $\mathrm{A}_{\mathrm{C}}=0,85$ & $\mathrm{~B}_{1}=0,95$ & $\mathrm{~B}_{2}=0,7705$ & $\mathrm{~B}_{1}=6700 \mathrm{MPa}^{-1}$ & $\mathrm{~B}_{2}=0,4 \mathrm{MPa}^{-1}$ \\
\hline $\mathrm{B}_{\mathrm{C}}=1050$ & $\beta_{1}=1,00 \mathrm{MPa}$ & $\beta_{2}=-10,00 \mathrm{MPa}$ & & \\
\hline$\varepsilon_{\mathrm{d} 0}=0,00007$ & \multicolumn{2}{|c|}{$\sigma_{\mathrm{f}}=3,50 \mathrm{MPa}$} & & \\
\hline
\end{tabular}




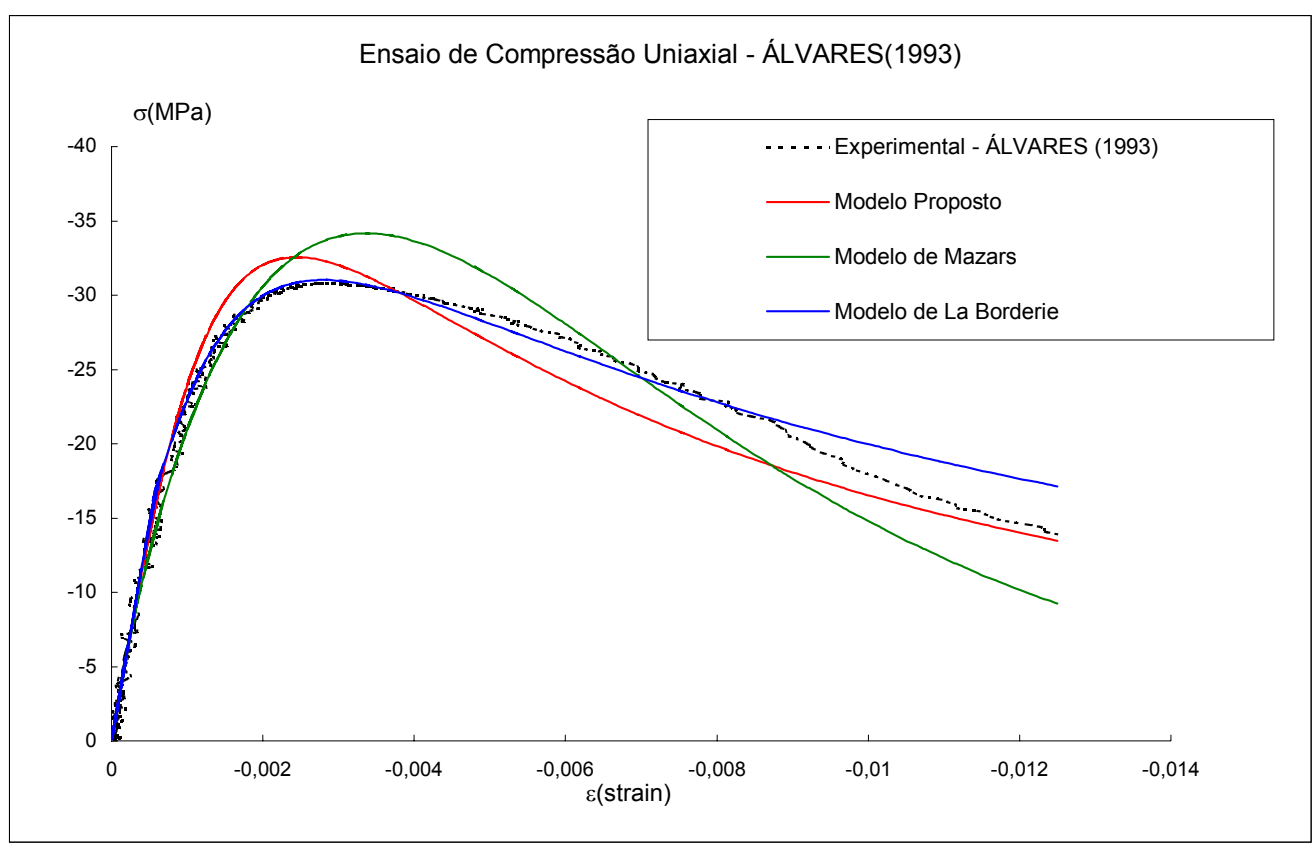

Figura 5.3 - Identificação paramétrica em compressão uniaxial: vigas em concreto armado

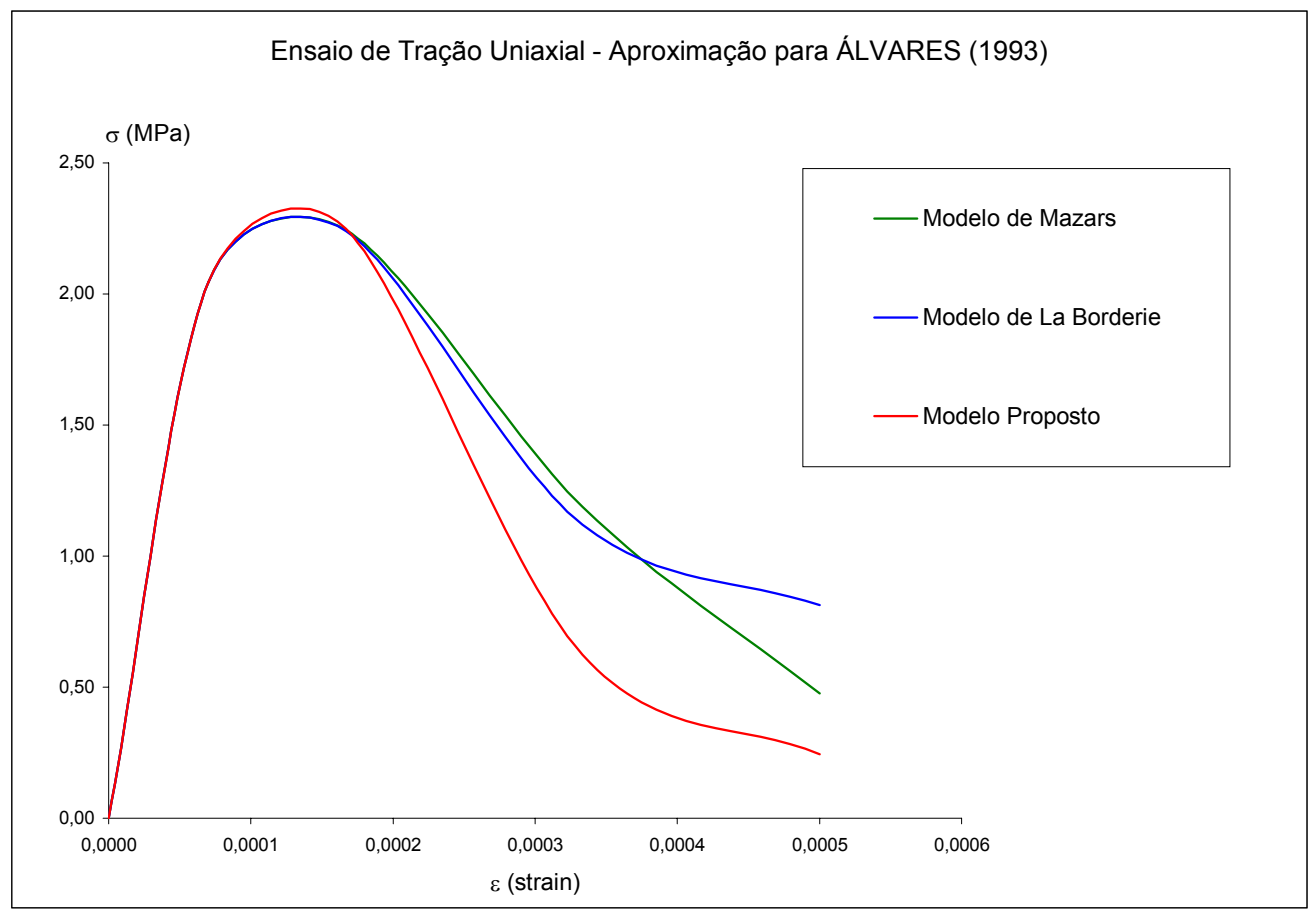

Figura 5.4 - Identificação paramétrica em tração uniaxial: vigas em concreto armado

Fazendo-se uso das simetrias de carregamento e de geometria, analisaram-se apenas as metades das vigas, impondo-se incrementos de deslocamentos durante a simulação numérica. Nas análises foram utilizados 20 elementos finitos, discretizaram-se as seções transversais em 15 camadas, sendo uma camada de aço e 
concreto na viga com $3 \phi 10.0 \mathrm{~mm}$, duas na viga com $5 \phi 10.0 \mathrm{~mm}$ e três na viga com $7 \phi 10.0 \mathrm{~mm}$, conforme a disposição das armaduras mostrada na Figura 5.2. Os confrontos entre os resultados numéricos e experimentais, mediante curvas carga aplicada (P) por deslocamento vertical no meio do vão das vigas estão ilustrados nas figuras 5.5 a 5.7 .

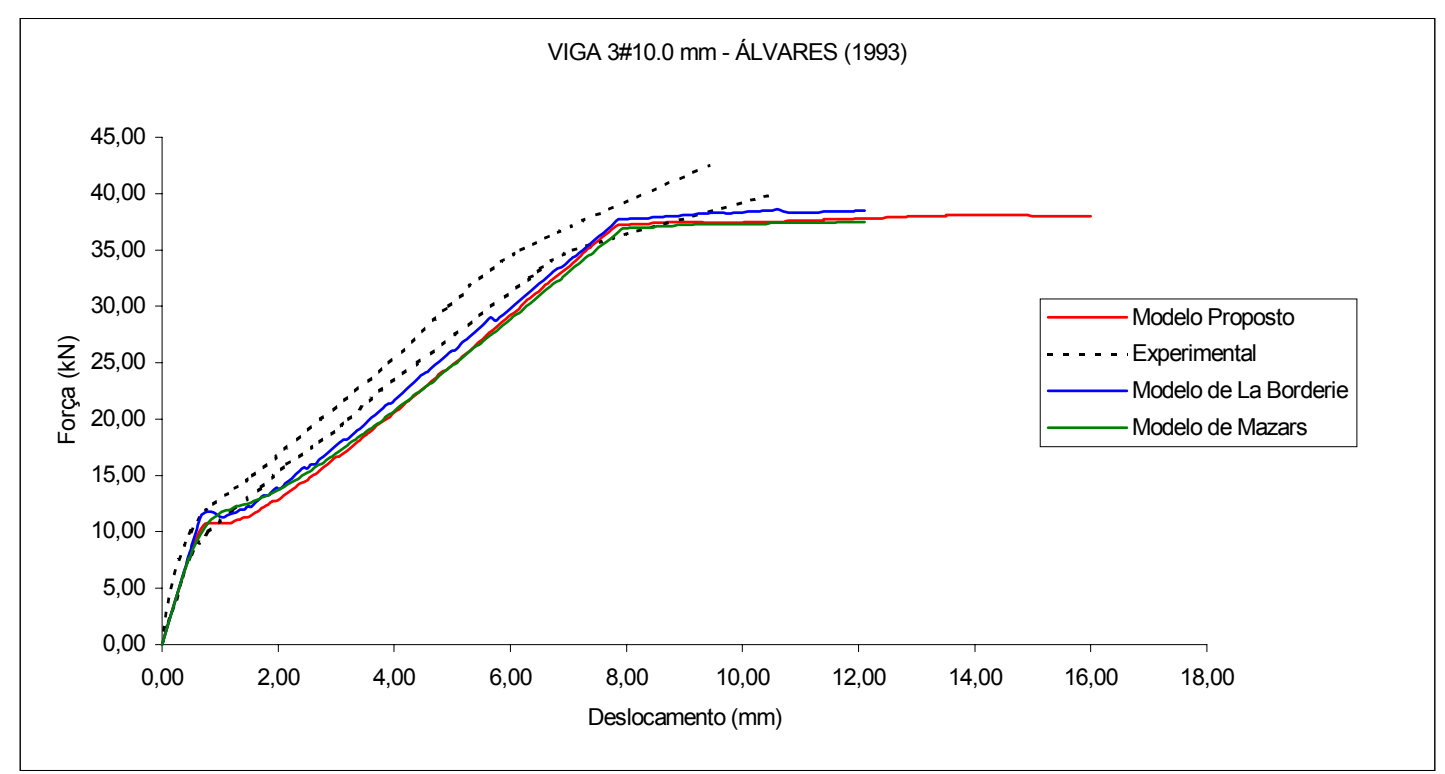

Figura 5.5 - Resultados numéricos da análise unidimensional - viga $3 \phi 10.0 \mathrm{~mm}$

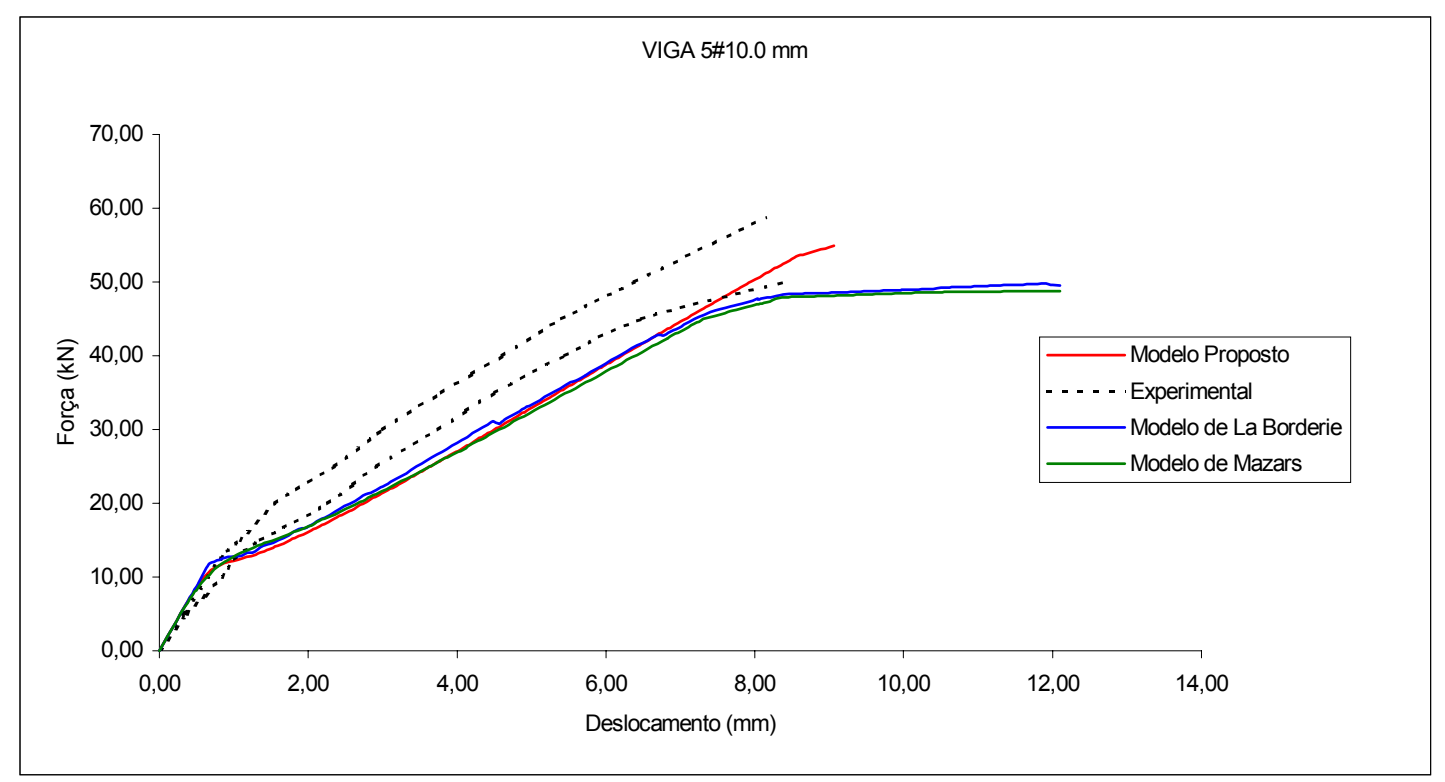

Figura 5.6 - Resultados numéricos da análise unidimensional - viga $5 \phi 10.0 \mathrm{~mm}$ 


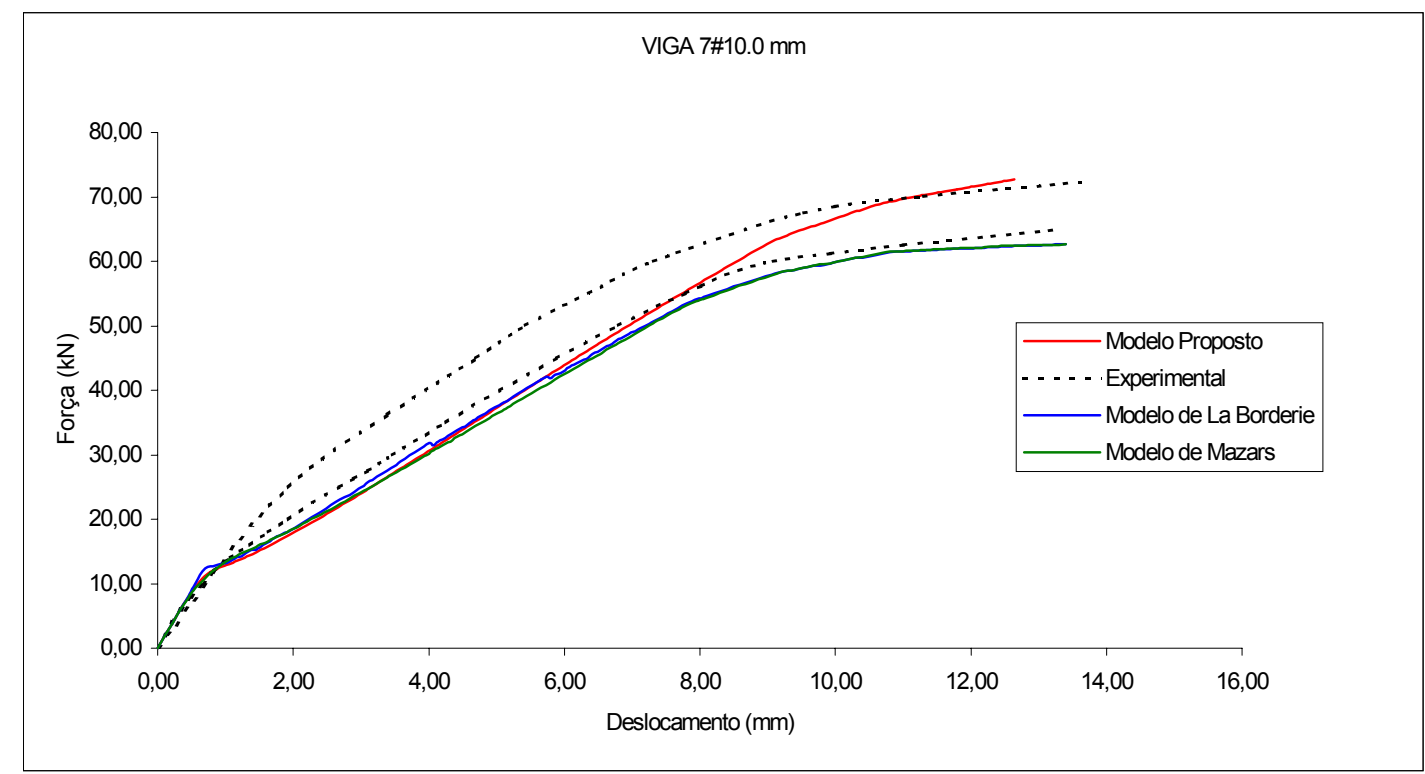

Figura 5.7 - Resultados numéricos da análise unidimensional - viga $7 \phi 10.0 \mathrm{~mm}$

Pode-se observar que as respostas numéricas tendem a reproduzir uma forte quebra de rigidez inicial das vigas seguida de uma recuperação de rigidez subseqüente à redistribuição de esforços. Os próprios resultados experimentais apontam que esse processo é mais evidente no caso da viga pouco armada em razão da menor interação entre concreto e armadura, o que leva a um panorama de fissuração mais localizado entre as forças aplicadas.

Em regime de serviço, observa-se ainda que os modelos de dano isótropo de Mazars e de La Borderie apresentam respostas similares às obtidas com o emprego do modelo proposto. Nesse regime, devido ao tipo simplificado de análise (unidimensional), não é possível verificar alguma possível vantagem do emprego do modelo proposto anisótropo sobre modelos de dano isótropo.

$\mathrm{Na}$ figura 5.8, ilustram-se as distribuições da danificação fornecidas pelos modelos em questão, na seção localizada no meio do vão da viga com $3 \phi 10.0 \mathrm{~mm}$. 

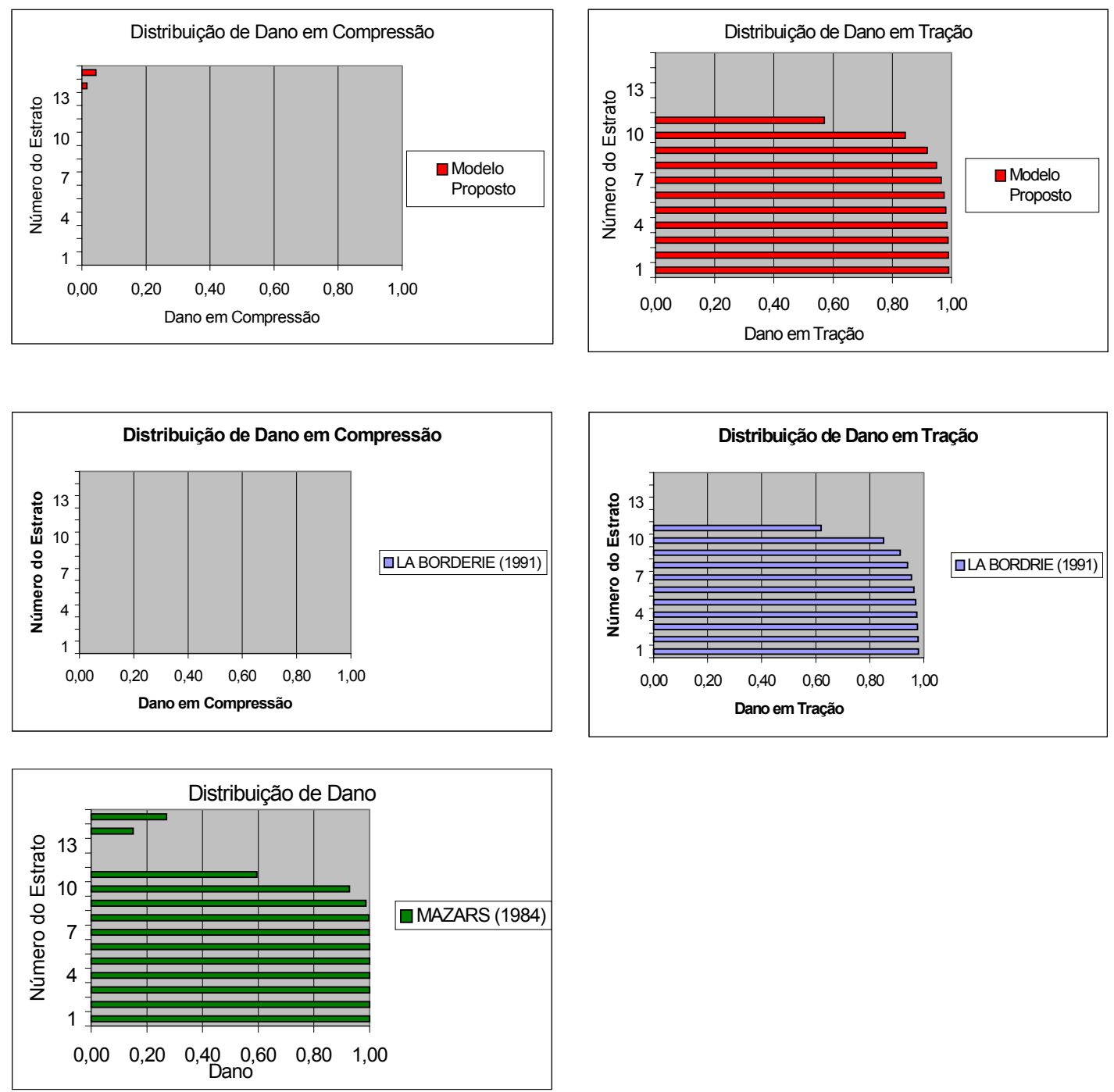

Figura 5.8 - Distribuição da danificação ao longo da seção transversal localizada no meio do vão (viga $3 \phi 10.0 \mathrm{~mm}-\mathrm{P}=30 \mathrm{kN}$ )

Já em regime próximo da ruptura os critérios de danificação adotados são um dos fatores contribuintes para as diferentes respostas entre os modelos. No modelo de Mazars o critério é expresso em termos de alongamentos equivalentes, enquanto que nos modelos de La Borderie e proposto neste trabalho, os critérios estão escritos em termos de energia. Além disso, um outro fator de influência é que o modelo proposto utiliza o princípio de equivalência de energia. Esses fatores implicam em evoluções diferentes para as variáveis de danificação.

Vale ainda comentar que no modelo proposto, como resultado da obtenção de uma danificação menos intensa, em regime de ruptura o concreto acaba por resistir aos esforços solicitantes de maneira mais eficiente, fazendo com que as armaduras 
das vigas com cinco e sete barras de aço venham a plastificar em níveis mais elevados de solicitação do que nos casos onde os modelos isótropos foram empregados. Ainda nesse contexto, observa-se que as respostas numéricas indicam que a plastificação das armaduras ocorrem em diferentes níveis de solicitação quando comparadas com as respostas experimentais, principalmente nos casos das vigas com cinco e sete barras de aço. Isso pode ser explicado pelo fato de ser assumida perfeita aderência entre a armadura e o concreto como hipótese para a simulação numérica.

Conclui-se que os resultados obtidos com a discretização adotada são bons quando comparados com a resposta experimental das vigas, com destaque para o modelo proposto.

\subsubsection{Pórtico em Concreto Armado [VECCHIO \& EMARA (1992)]}

As características geométricas do pórtico, assim como a distribuição das armaduras estão ilustradas na Figura 5.9. O concreto utilizado na confecção do pórtico tem módulo de elasticidade $\mathrm{E}_{\mathrm{c}}=30400 \mathrm{MPa}$; o aço possui $\mathrm{E}_{\mathrm{a}}=192500 \mathrm{MPa}$, tensão de início de plastificação de $418 \mathrm{MPa}$ e tensão última de $596 \mathrm{MPa}$. Foi adotado um modelo elasto-plástico bilinear com um módulo de elasticidade reduzido no segundo trecho: $\mathrm{E}_{\mathrm{a} 2}=0,009 \mathrm{E}_{\mathrm{a}}$.

No ensaio experimental inicialmente aplicou-se uma força axial total de 700 $\mathrm{kN}$ para cada coluna, mantida então constante durante toda a aplicação da força lateral. Esta força foi aplicada em estágios até a capacidade última do pórtico ser atingida. Maiores detalhes encontram-se em VECCHIO \& EMARA (1992). 


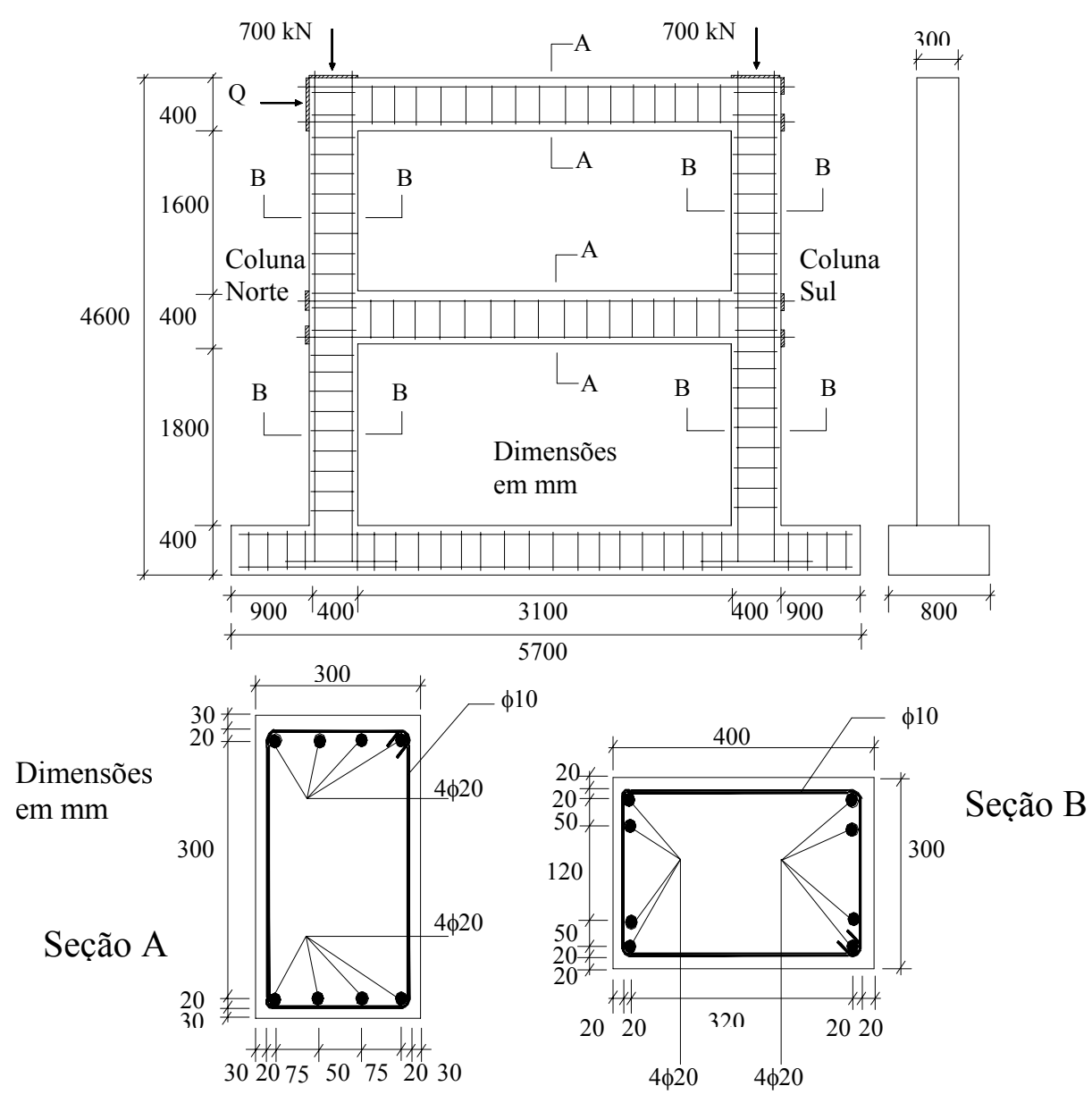

Figura 5.9 - Geometria e armação do pórtico

Os parâmetros de compressão dos modelos testados foram identificados a partir de ensaios experimentais de corpos de prova em compressão uniaxial, realizados por VECCHIO \& EMARA (1992). Por outro lado, para os parâmetros de tração tanto do modelo de Mazars quanto do proposto foram adotados aqueles mesmos utilizados no exemplo anterior, por se tratarem de valores médios representativos para concretos normais. Contudo, vale ressaltar que os parâmetros do modelo de La Borderie foram alterados com o objetivo de simular o comportamento do pórtico durante a fase de descarregamento.

$\mathrm{Na}$ tabela 5.2 e nas figuras 5.10 e 5.11 são fornecidos os valores dos parâmetros utilizados em cada modelo, além das respostas numéricas nos ensaios uniaxiais. 
Tabela 5.2 - Parâmetros do modelo de Mazars, de La Borderie e do proposto

\begin{tabular}{|l|l|l|l|l|}
\hline \multirow{2}{*}{ Mazars } & \multicolumn{2}{|c|}{ La Borderie } & \multicolumn{2}{c|}{ Proposto } \\
\cline { 2 - 5 } & $\begin{array}{c}\text { Parâmetros } \\
\text { de Tração }\end{array}$ & $\begin{array}{c}\text { Parâmetros de } \\
\text { Compressão }\end{array}$ & $\begin{array}{c}\text { Parâmetros de } \\
\text { Tração }\end{array}$ & $\begin{array}{c}\text { Parâmetros de } \\
\text { Compressão }\end{array}$ \\
\hline $\mathrm{A}_{\mathrm{T}}=0,995$ & $\mathrm{Y}_{01}=2,25 \times 10^{-4} \mathrm{MPa}$ & $\mathrm{Y}_{02}=0,5 \times 10^{-2} \mathrm{MPa}$ & $\mathrm{Y}_{01}=0,72 \times 10^{-4} \mathrm{MPa}$ & $\mathrm{Y}_{02}=1,7 \times 10^{-3} \mathrm{MPa}$ \\
\hline $\mathrm{B}_{\mathrm{T}}=8000$ & $\mathrm{~A}_{1}=2,78 \times 10^{+3} \mathrm{MPa}^{-1}$ & $\mathrm{~A}_{2}=14,00 \mathrm{MPa}^{-1}$ & $\mathrm{~A}_{1}=50$ & $\mathrm{~A}_{2}=-0,8$ \\
\hline $\mathrm{A}_{\mathrm{C}}=1,30$ & $\mathrm{~B}_{1}=0,93$ & $\mathrm{~B}_{2}=0,90$ & $\mathrm{~B}_{1}=6700 \mathrm{MPa}^{-1}$ & $\mathrm{~B}_{2}=1,1 \mathrm{MPa}^{-1}$ \\
\hline $\mathrm{B}_{\mathrm{C}}=1890$ & $\beta_{1}=0,50 \mathrm{MPa}$ & $\beta_{2}=-2,00 \mathrm{MPa}$ & & \\
\hline$\varepsilon_{\mathrm{d} 0}=0,00007$ & \multicolumn{2}{|c|}{$\sigma_{\mathrm{f}}=3,50 \mathrm{MPa}$} & & \\
\hline
\end{tabular}

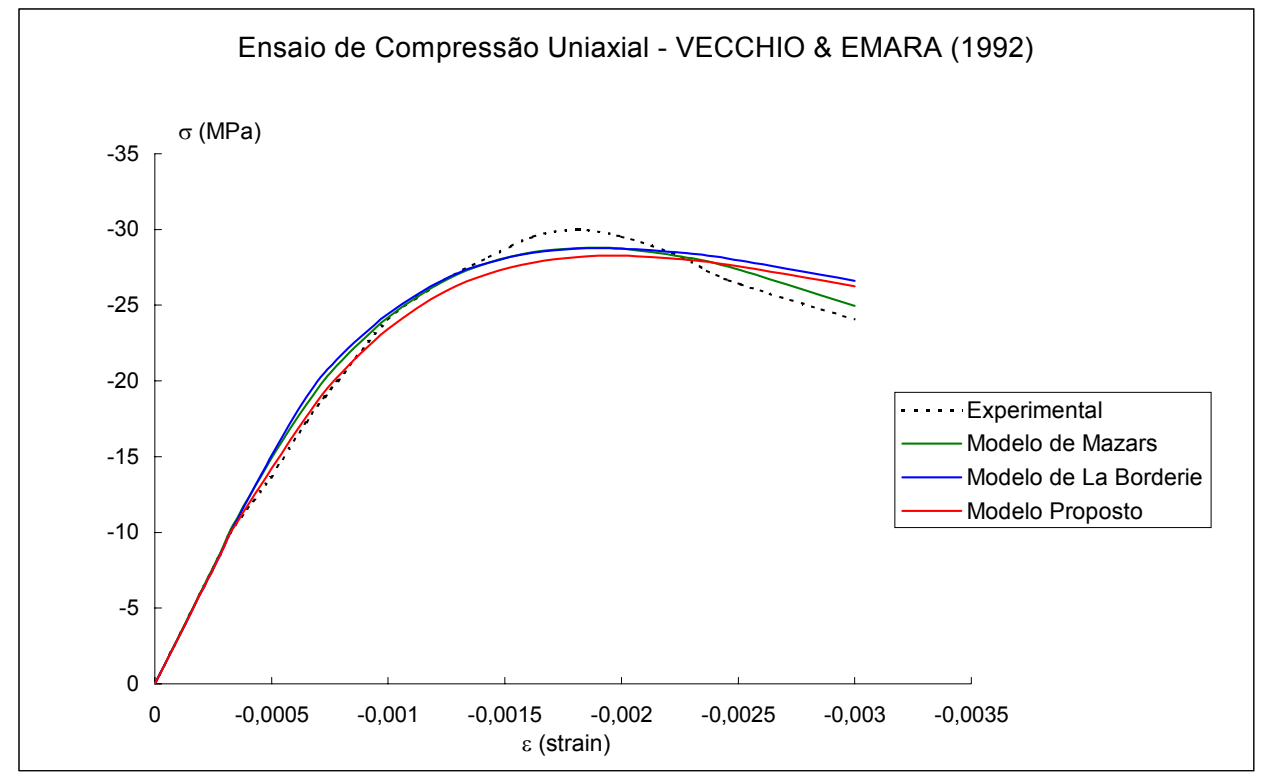

Figura 5.10 - Identificação paramétrica em compressão uniaxial: pórtico em concreto armado

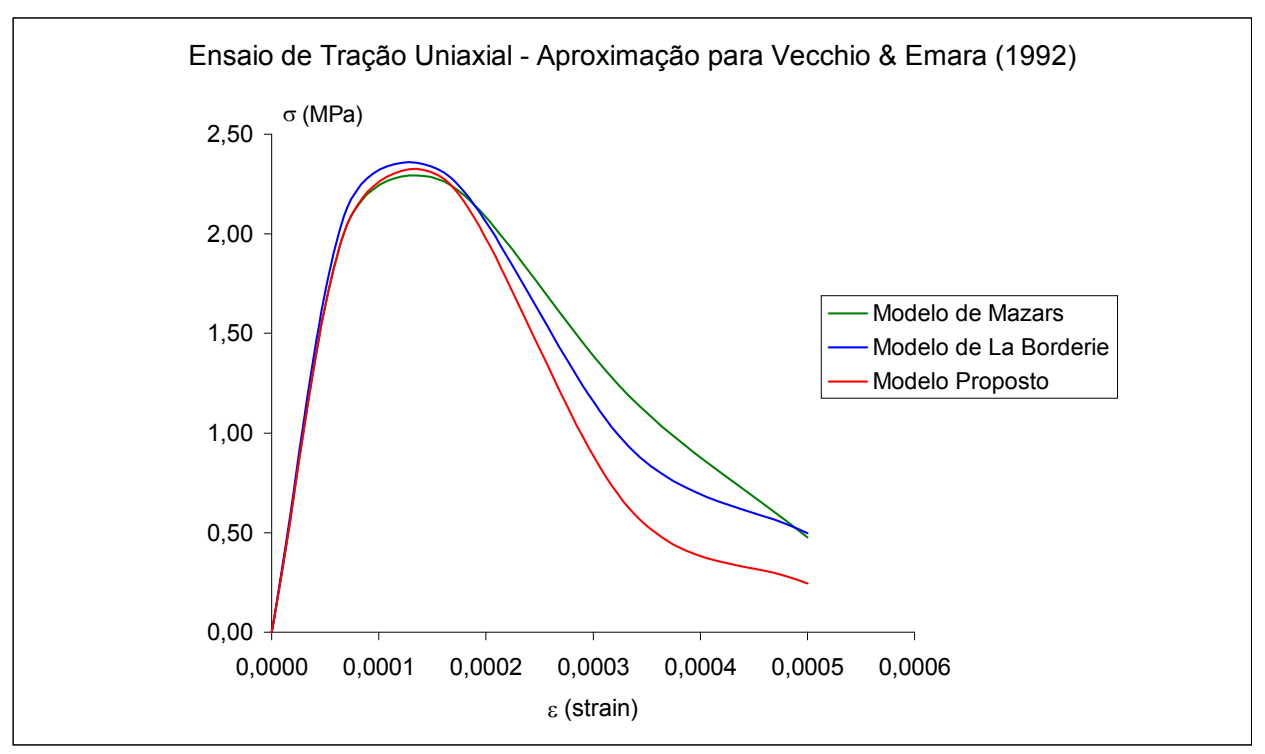

Figura 5.11 - Identificação paramétrica em tração uniaxial: pórtico em concreto armado 
Para a análise numérica foram impostos incrementos de deslocamentos no ponto de aplicação da força horizontal. Utilizaram-se 30 elementos finitos na discretização, sendo empregados 10 por coluna e 5 elementos por viga. As seções transversais foram estratificadas em 10 camadas. Observa-se que em PITUBA, PROENÇA \& ÁLVARES (1999) apresenta-se um estudo sobre o refinamento da discretização da seção transversal objetivando-se identificar uma possível influência do número de estratos nas respostas numéricas. As camadas de aço foram definidas em número de duas, de acordo com as disposições das armaduras nas seções (Figura 5.9).

Na Figura 5.12 apresentam-se em gráficos as respostas numéricas obtidas confrontadas com a experimental. Os gráficos representam a relação entre força horizontal aplicada e deslocamento horizontal no andar superior do pórtico.

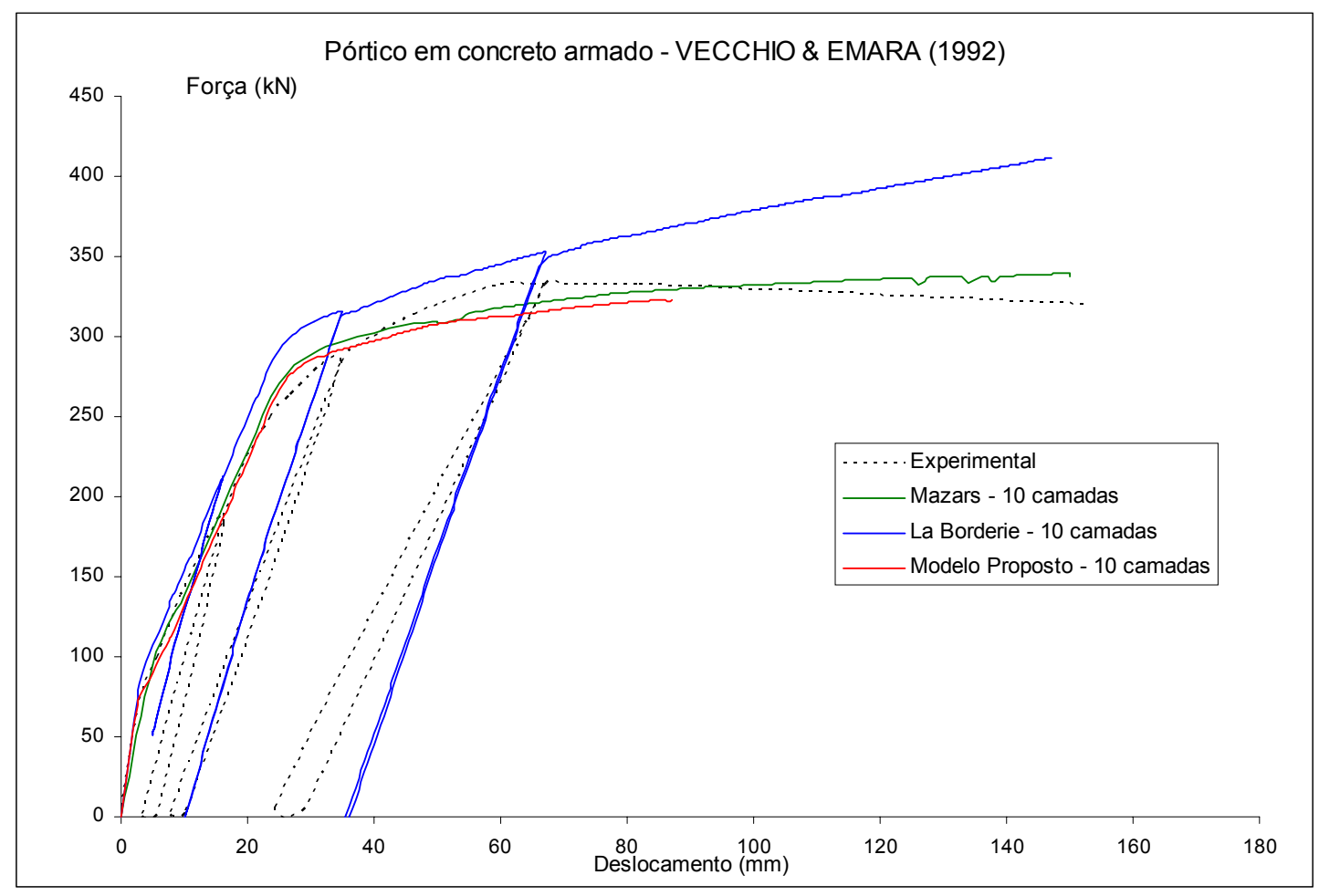

Figura 5.12 - Resultados numéricos do pórtico em concreto armado

Os resultados obtidos pelos modelos são julgados satisfatórios apesar da limitada identificação paramétrica. Por permitir considerar deformações permanentes na sua formulação, reproduziram-se com o modelo de La Borderie inclusive os 
deslocamentos residuais gerados; já com os outros modelos o caminho de descarregamento apontaria para a origem do gráfico, o que não representa a realidade experimental.

Segundo PITUBA, PROENÇA \& ÁlVARES (1999), analisando-se a resposta global da estrutura ensaiada (sem considerar as etapas de descarregamento) com o modelo de Mazars, o refinamento da discretização em camadas não leva a uma mudança significativa do resultado. Isto talvez seja consequência da definição de uma única variável de dano, que combina os efeitos de danificação em tração e compressão, assumindo desde logo, valor muito alto em todas as camadas dos elementos mais solicitados. Entretanto, no modelo de La Borderie há a definição de duas variáveis independentes de dano, e com o refinamento da discretização em camadas é possível colher, de uma forma melhor, a influência da danificação na seção. $\mathrm{O}$ mesmo acontece com o modelo proposto, porém a diferença em relação ao modelo de La Borderie é a possibilidade de se colher uma boa resposta numérica, sem a necessidade de um custo computacional maior com o refinamento da discretização.

Em VECCHIO \& EMARA (1992) relata-se que o pórtico evidenciou um primeiro estágio de fissuração com força lateral de $52,50 \mathrm{kN}$. Na simulação com o modelo de Mazars o pórtico apresentou uma primeira perda de rigidez ao atingir a força de 92,58 kN. Com o modelo de La Borderie a mesma perda se manifestou com a força de 91,06 kN. Já com o modelo proposto a força obtida foi de 45,58 kN. Considera-se que a previsão de início de fissuração pelo modelo proposto está de acordo com a realidade experimental.

Numa etapa posterior da análise numérica, o modelo de Mazars indicou uma força de $260 \mathrm{kN}$ em correspondência à primeira plastificação de armaduras. O mesmo fenômeno aconteceu com La Borderie em 263,90 kN e com o modelo proposto em 254,8 kN. A força última observada durante o experimento foi de 332 kN. Na simulação numérica, tanto com Mazars quanto com La Borderie, o pórtico suportaria ulteriores incrementos de deslocamentos, porém com Mazars evidenciouse uma força máxima de $340,10 \mathrm{kN}$. A força máxima obtida com o modelo proposto foi de $323 \mathrm{kN}$. 
Pode-se concluir, pelos exemplos unidimensionais apresentados, pela validade do emprego do modelo proposto em combinação com a técnica de discretização adotada na simulação do comportamento de estruturas lineares em concreto armado.

\subsection{ANÁLISES BIDIMENSIONAIS}

Uma versão bidimensional do modelo proposto foi implementada num código de cálculo em elementos finitos para análises planas. Nestas análises, apenas o concreto possui comportamento não-linear, para o aço admite-se uma relação constitutiva linear. Com relação à interação entre os dois materiais, admitiu-se perfeita aderência entre o aço e o concreto.

Fazendo-se uso das simetrias de carregamento e de geometria, discretizou-se, portanto, apenas uma metade das vigas, conforme ilustra a figura 5.13.

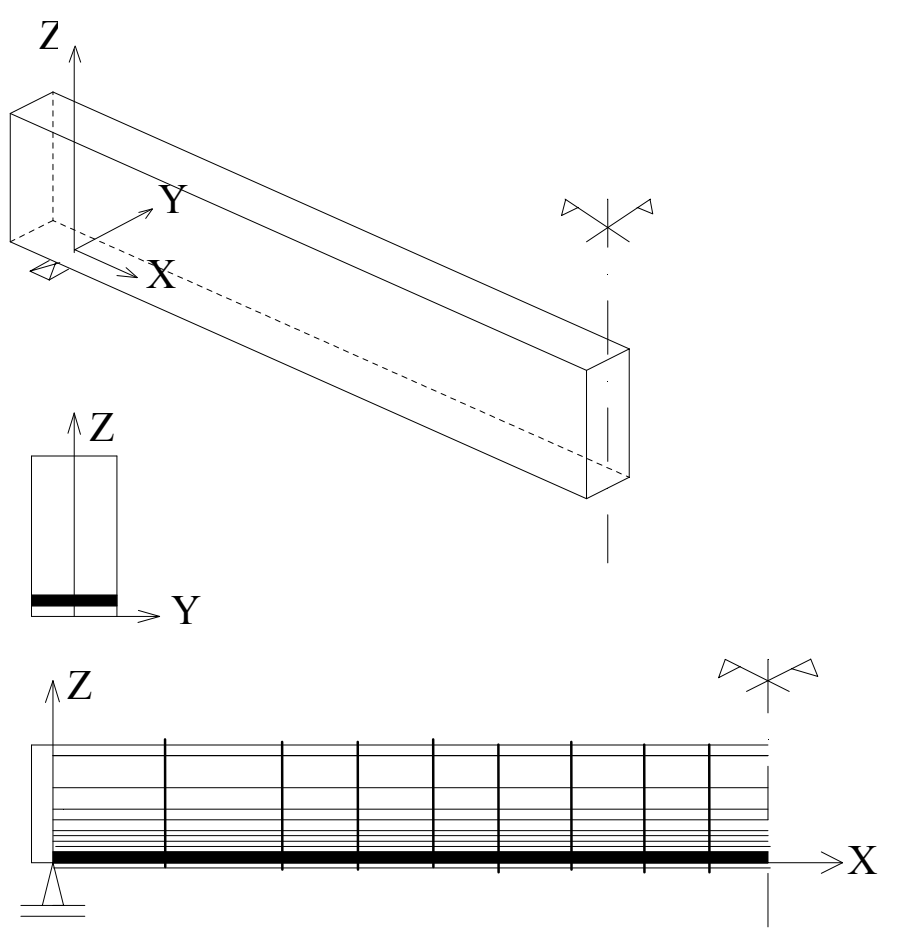

Figura 5.13 - Discretização em elementos finitos bidimensionais

De acordo com o objetivo de avaliar a resposta numérica fornecida pelo modelo proposto em análises planas, com o emprego do método dos elementos 
finitos, dois aspectos de interesse são enfatizados: a verificação de eventuais problemas numéricos ligados a este tipo de discretização e a capacidade do modelo em reproduzir a zona de distribuição de dano de acordo com a fissuração evidenciada experimentalmente.

\subsubsection{Viga em Concreto Armado [PEREGO (1989)]}

Trata-se de uma viga em concreto armado analisada por PEREGO (1989), que possui $5,00 \mathrm{~m}$ de vão livre e apresenta uma seção transversal retangular de dimensão 20 x $50 \mathrm{~cm}$. A armadura longitudinal é constituída por 6 barras de $32 \mathrm{~mm}$ de diâmetro, posicionadas em três camadas na zona inferior da seção, e por 2 barras de diâmetro de $8 \mathrm{~mm}$, dispostas na parte superior e com função construtiva. $\mathrm{Na}$ confecção da viga foi utilizado um concreto com $\mathrm{E}_{\mathrm{c}}=24700 \mathrm{MPa}$ e aço das armaduras com $\mathrm{E}_{\mathrm{a}}=210000 \mathrm{MPa}$. Na figura 5.14 são fornecidos os detalhes da geometria da viga.

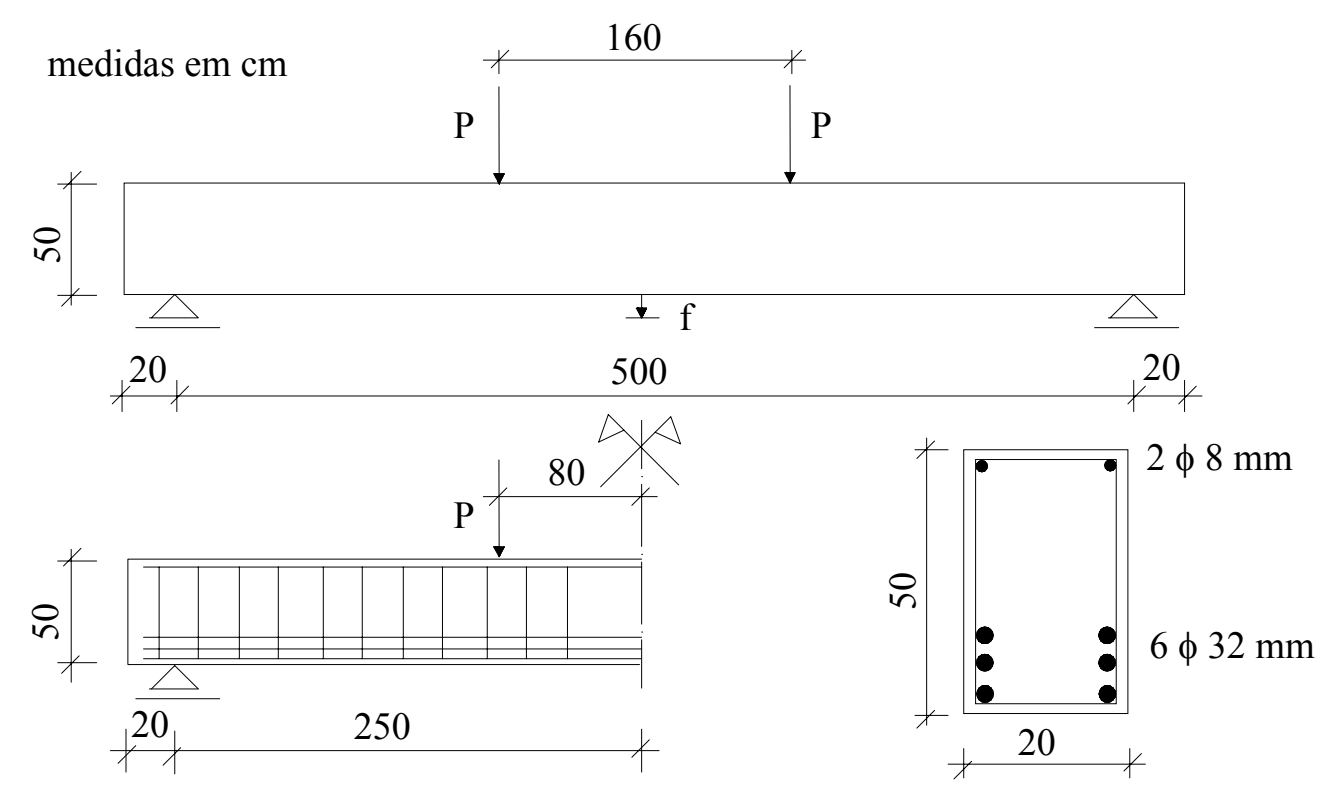

Figura 5.14 - Viga em concreto armado: geometria, simetria geométrica e de carregamento e disposição da armadura

Neste exemplo, para os parâmetros do modelo proposto relativos a $\mathrm{D}_{1}$ e $\mathrm{D}_{2}$, foram adotados os mesmos valores utilizados no estudo das vigas do item 5.2.1. Na análise plana é necessária também a obtenção dos parâmetros relativos à lei de evolução da variável $\mathrm{D}_{3}$, os quais foram identificados como base na resposta 
ilustrada na figura 4.6 do capítulo anterior. Para efeito de comparação de resultados, além das medidas experimentais apresenta-se a resposta numérica obtida por PEREGO (1989) com o modelo de Mazars. A tabela a seguir resume os parâmetros utilizados pelos modelos envolvidos na análise.

Tabela 5.3 - Parâmetros dos modelos de Mazars [PEREGO (1989)] e proposto.

\begin{tabular}{|l|l|l|l|l|}
\hline \multicolumn{2}{|c|}{ Mazars } & \multicolumn{3}{c|}{ Proposto } \\
\hline $\begin{array}{c}\text { Parâmetros de } \\
\text { Tração }\end{array}$ & $\begin{array}{c}\text { Parâmetros de } \\
\text { Compressão }\end{array}$ & $\begin{array}{c}\text { Parâmetros de } \\
\text { Tração }\end{array}$ & Parâmetros de Compressão \\
\hline $\mathrm{A}_{\mathrm{T}}=0,995$ & $\mathrm{~A}_{\mathrm{C}}=1,13$ & $\mathrm{Y}_{01}=0,72 \times 10^{-4} \mathrm{MPa}$ & $\mathrm{Y}_{02}=0,5 \times 10^{-3} \mathrm{MPa}$ & $\mathrm{Y}_{03}=0,5 \times 10^{-3} \mathrm{MPa}$ \\
\hline $\mathrm{B}_{\mathrm{T}}=8000$ & $\mathrm{~B}_{\mathrm{C}}=1643,5$ & $\mathrm{~A}_{1}=50$ & $\mathrm{~A}_{2}=-0,9$ & $\mathrm{~A}_{3}=-0,6$ \\
\hline \multicolumn{2}{|c|}{$\varepsilon_{\mathrm{d} 0}=0,000067$} & $\mathrm{~B}_{1}=6700 \mathrm{MPa}^{-1}$ & $\mathrm{~B}_{2}=0,4 \mathrm{MPa}^{-1}$ & $\mathrm{~B}_{3}=70000 \mathrm{MPa}^{-1}$ \\
\hline
\end{tabular}

Alguns comentários adicionais devem ser feitos em relação à discretização e idealização adotadas.

Com relação ao comportamento elástico-linear para o aço, tal hipótese é justificável no caso de vigas que possuem alta taxa de armadura, onde o colapso acontece sobretudo pelo comprometimento do concreto.

A discretização da viga foi efetuada utilizando-se uma malha constituída por 342 elementos finitos quadrangulares de 4 nós, dispostos no plano médio XZ (Fig. 5.13). A altura da viga foi subdividida em 38 camadas de elementos onde uma única camada representa a armadura, com área equivalente disposta no baricentro geométrico das barras de aço.

A força concentrada aplicada foi representada por uma carga distribuída em torno do ponto de aplicação para evitar qualquer problema de concentração exagerada de tensões nesta zona. $\mathrm{O}$ mesmo procedimento foi adotado na simulação do apoio da viga.

A resposta obtida em termos da curva carga-deslocamento (Fig. 5.15) mostra, em sua maior parte, uma ótima coerência com os resultados experimentais apresentados em PEREGO (1989). De fato, o modelo proposto é capaz de colher com boa precisão a não-linearidade da curva até o nível de força de $220 \mathrm{kN}$, a partir do qual surge uma instabilidade na resposta numérica, decorrente dos elevados níveis de danificação. Na análise efetuada por PEREGO (1989), com o modelo de Mazars, apresentou-se a instabilidade numérica já por volta da força de $120 \mathrm{kN}$. 
Observa-se mais uma vez, que o modelo proposto penaliza seletivamente a rigidez do material de acordo com a direção considerada. Isso não ocorre com o modelo de Mazars, que por danificar exageradamente a rigidez do material em todas as direções em torno de um ponto, acaba por apresentar problemas de ordem numérica devido ao mal condicionamento da matriz de rigidez global da estrutura, logo num nível intermediário de carga.

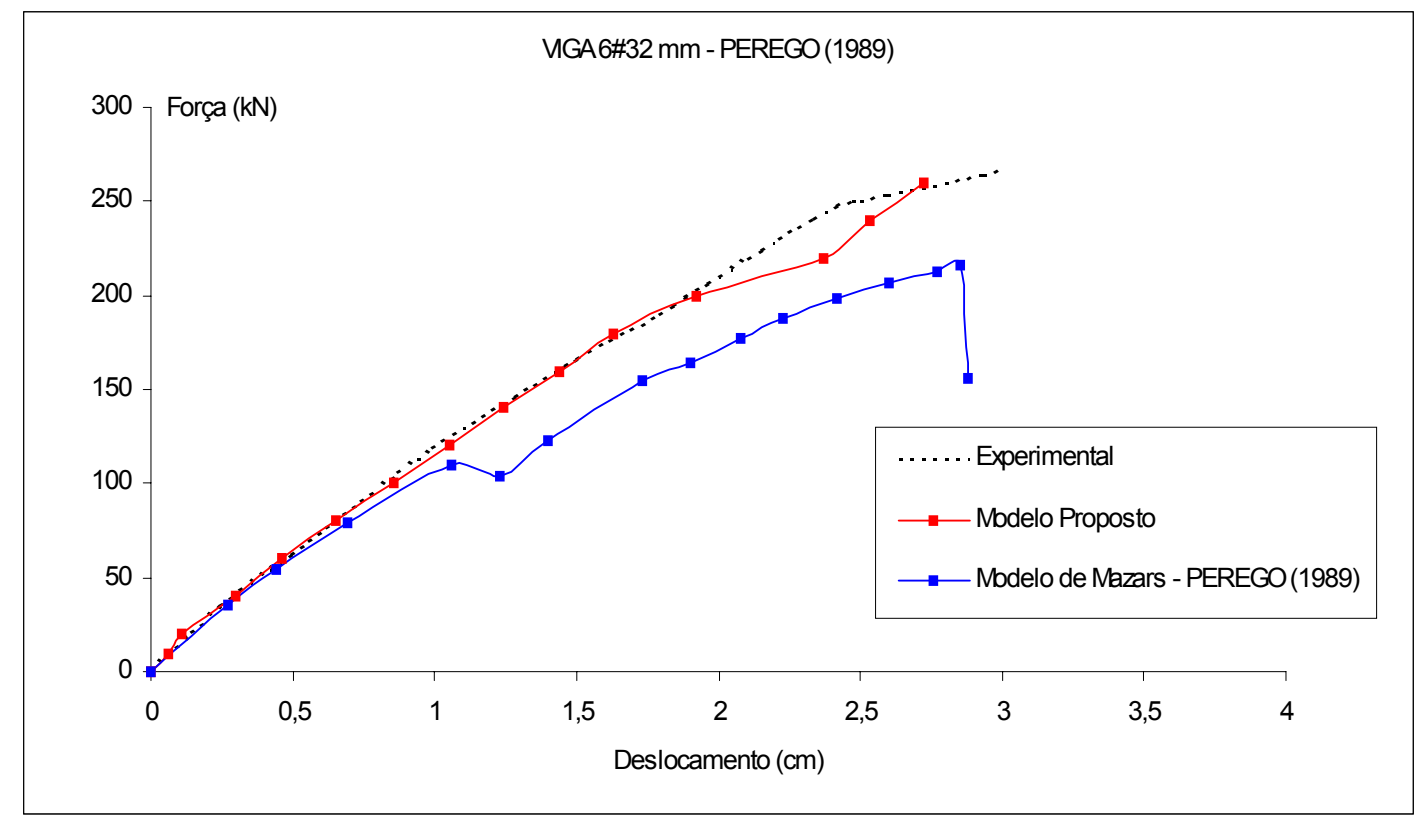

Figura 5.15 - Resultados numéricos - viga $6 \phi 32.0 \mathrm{~mm}$ :

curva carga-deslocamento no meio do vão

É possível dar uma explicação plausível para a instabilidade observada. O modelo numérico na verdade procura reproduzir uma situação de danificação fortemente localizada, que seria equivalente à formação de uma zona de fissuração intensa. Fisicamente essa zona se manifesta efetivamente entre as forças aplicadas.

Para visualizar a distribuição de dano na viga, representam-se curvas de isodano (curvas caracterizadas por $\mathrm{D}=$ constante), obtidas com base na interpolação dos valores das variáveis de dano nos pontos de integração adotados. Deve-se observar que esta representação é mais eficiente quanto maior for o número de pontos adotados. Nas figuras a seguir são ilustradas as curvas de isodano para as diferentes variáveis de dano do modelo proposto e em diversos instantes da história de carregamento. 

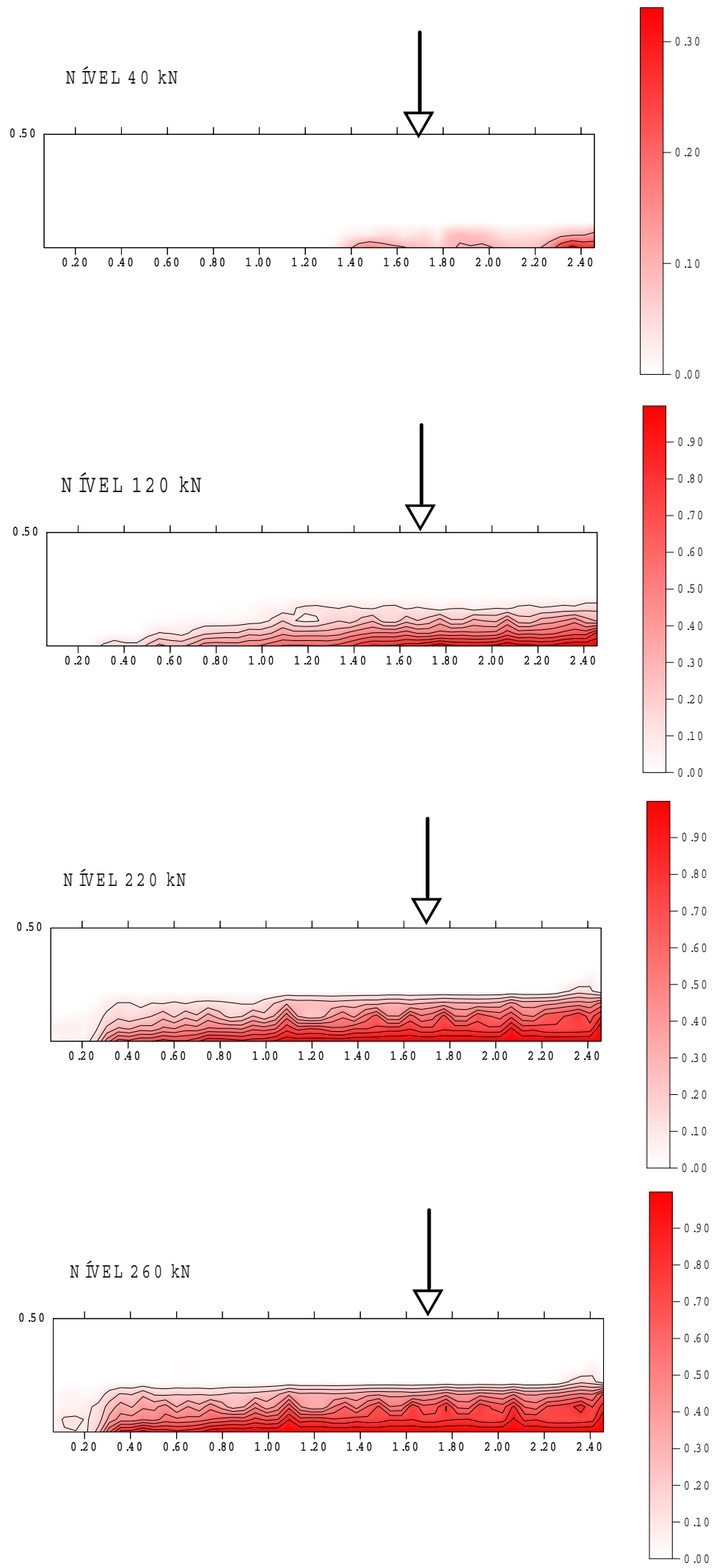

Figura 5.16 - Viga em concreto armado - viga $6 \phi 32.0 \mathrm{~mm}$ : distribuição de dano $\mathrm{D}_{1}$ 

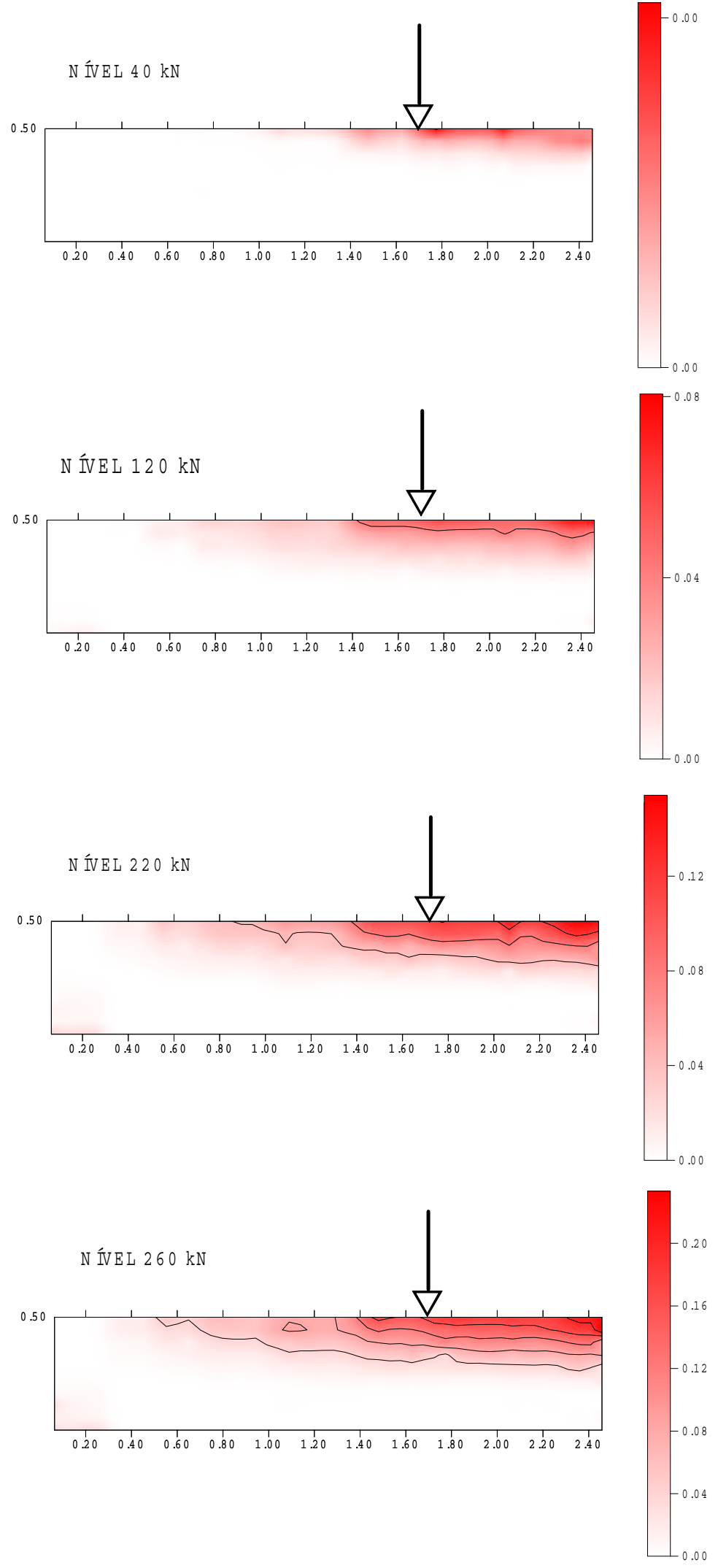

Figura 5.17 - Viga em concreto armado - viga $6 \phi 32.0 \mathrm{~mm}$ : distribuição de dano $\mathrm{D}_{2}$ 

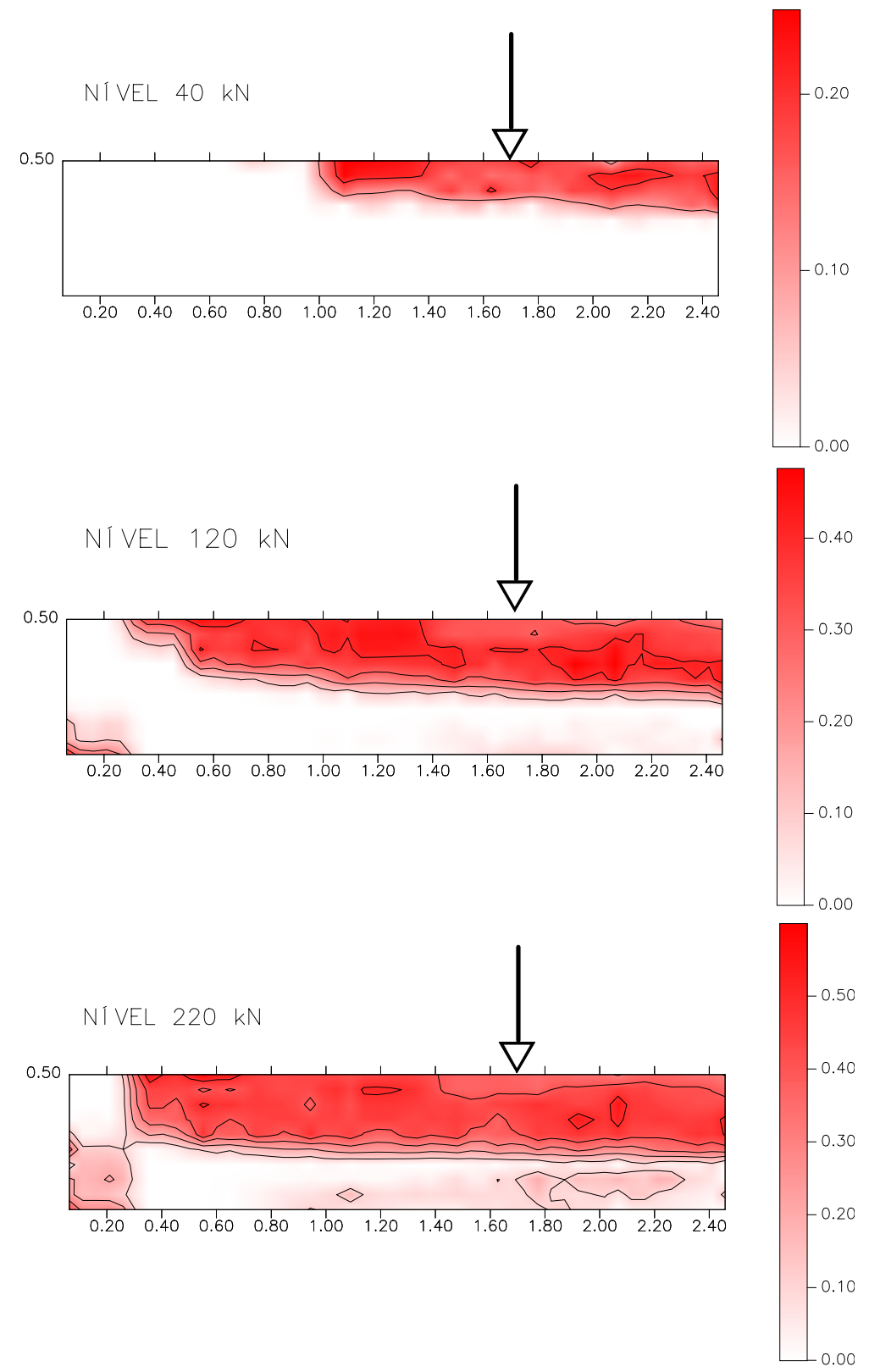

Figura 5.18 - Viga em concreto armado - viga $6 \phi 32.0 \mathrm{~mm}$ :

Distribuição de dano $\mathrm{D}_{3}$

Como se pode observar, já para um nível de solicitação correspondente a 15\% da força máxima, tem-se uma considerável danificação em tração na parte inferior da viga. Nos sucessivos níveis de carregamento observa-se que a zona de dano em tração se propaga de baixo para cima e em direção à zona do apoio. $\mathrm{Na}$ região superior da viga, valores de dano em compressão $\mathrm{D}_{2}$ surgem somente por volta de $50 \%$ da força máxima. Próximo à intensidade de $220 \mathrm{kN}$ para a força máxima aplicada observa-se uma zona de dano $\mathrm{D}_{3}$ com razoáveis valores, inclusive indicando 
uma certa concentração de danificação junto ao apoio, e uma vasta região inferior com dano $\mathrm{D}_{1}$ chegando a atingir valores próximos a 1 . Esta evolução de danificação está de acordo com o exposto em PEREGO (1989).

\subsubsection{Vigas em Concreto Armado [ÁLVARES (1993)]}

As vigas analisadas no item 5.2.1 com aplicação do modelo proposto em sua versão uniaxial, passam agora a ser estudadas com o emprego de uma versão para análise plana. Portanto os parâmetros elásticos do concreto e do aço são mantidos. $\mathrm{O}$ mesmo acontece com os parâmetros do modelo proposto, relativos às leis de evolução das variáveis de dano $\mathrm{D}_{1}$ e $\mathrm{D}_{2}$.

Em sua versão bidimensional, o modelo utiliza a variável de dano $\mathrm{D}_{3}$ e os valores adotados para os parâmetros envolvidos com essa variável são os mesmos utilizados no exemplo anterior (item 5.3.1). Para efeito de comparação, apresentamse nos gráficos de resultados além da reposta experimental, a resposta numérica obtida com o modelo em sua versão uniaxial. A tabela a seguir contém os valores dos parâmetros para o modelo proposto.

Tabela 5.4 - Parâmetros do modelo proposto.

\begin{tabular}{|l|c|l|}
\hline \multicolumn{3}{|c|}{ Proposto } \\
\hline $\begin{array}{c}\text { Parâmetros de } \\
\text { Tração }\end{array}$ & \multicolumn{2}{|c|}{ Parâmetros de Compressão } \\
\hline $\mathrm{Y}_{01}=0,72 \times 10^{-4} \mathrm{MPa}$ & $\mathrm{Y}_{02}=0,5 \times 10^{-3} \mathrm{MPa}$ & $\mathrm{Y}_{03}=0,5 \times 10^{-3} \mathrm{MPa}$ \\
\hline $\mathrm{A}_{1}=50$ & $\mathrm{~A}_{2}=-0,9$ & $\mathrm{~A}_{3}=-0,6$ \\
\hline $\mathrm{B}_{1}=6700 \mathrm{MPa}^{-1}$ & $\mathrm{~B}_{2}=0,4 \mathrm{MPa}^{-1}$ & $\mathrm{~B}_{3}=70000 \mathrm{MPa}^{-1}$ \\
\hline
\end{tabular}

Os primeiros testes realizados com malhas formadas por 342 elementos finitos quadrangulares de 4 nós dispostos no plano médio XZ (Fig. 5.13), aproveitando-se a simetria das vigas, não apresentaram resultados satisfatórios. De fato, notou-se tanto irregularidades nas curvas-resposta quanto rigidez exagerada.

O problema de irregularidade da resposta foi contornado adotando-se uma alternativa eficaz porém com custo computacional crescente com o avanço da análise. Trata-se da não contribuição para a atualização da matriz de rigidez global da estrutura, somente para fins de reaplicação do resíduo, de pontos que apresentam certo nível de dano $(0,65$ por exemplo). 
Já o problema de travamento dos elementos, que poderia ser em parte responsável pela rigidez excessiva, pôde ser contornado com o emprego de uma discretização mais refinada na direção longitudinal, diminuindo assim a diferença entre a espessura e o comprimento dos elementos representativos do aço em relação aos demais. Para tanto, adotou-se uma discretização composta por 7200 elementos finitos quadrangulares de 4 nós subdividindo a altura da viga em 60 camadas. A figura 5.19 ilustra o refinamento da malha e a simulação da distribuição de apoios e cargas na estrutura.

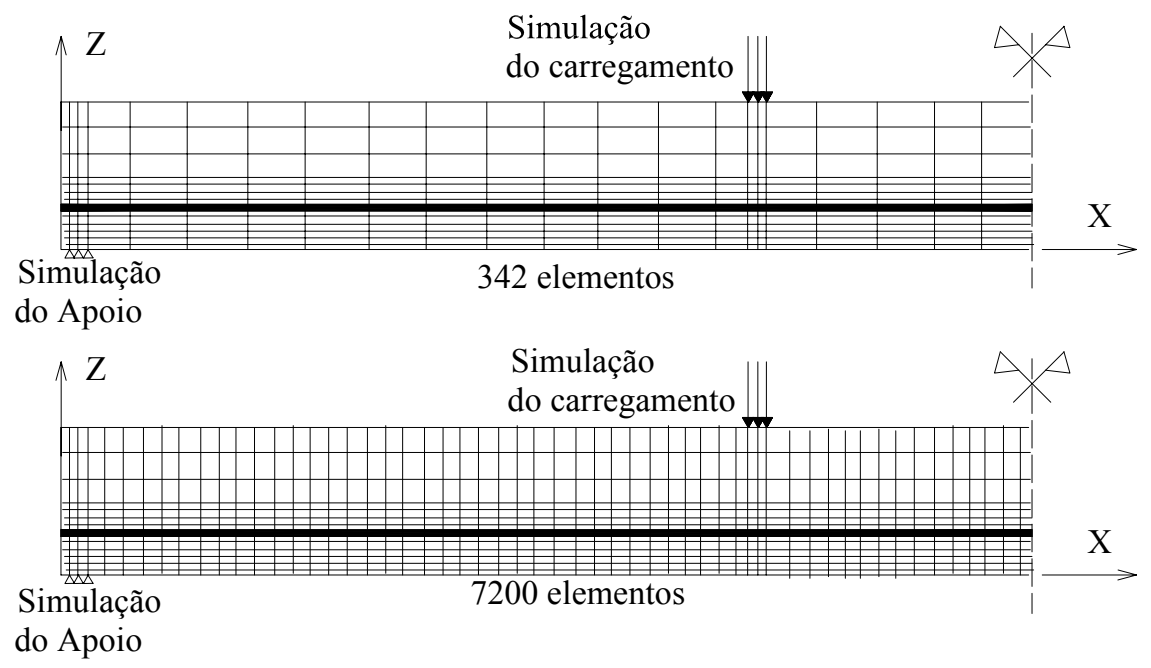

Figura 5.19 - Configurações das malhas com 342 e 7200 elementos finitos

As figuras 5.20, 5.21 e 5.22 ilustram as respostas obtidas. Observa-se que nas vigas super armada e normalmente armada a resposta numérica consegue simular bem o comportamento experimental até níveis intermediários de carga. Já o mesmo não acontece no caso da viga de $3 \# 10.0 \mathrm{~mm}$. Acredita-se que a incorporação de leis de evolução e a identificação paramétrica das variáveis de dano relativas ao cisalhamento possam contribuir para melhorar as respostas nesse caso. De fato, a danificação relativa ao cisalhamento, gerando uma contribuição importante na dissipação de energia, é possível de ser fornecida pelo modelo proposto, e potencialmente visa melhorar sua capacidade de simular o comportamento do concreto em situações onde haja uma perda mais intensa de resistência ao cisalhamento. Contudo, é importante observar que mesmo essa consideração pode ser limitada se houver forte localização da danificação, o que ocorre próximo da situação de carregamento último. 


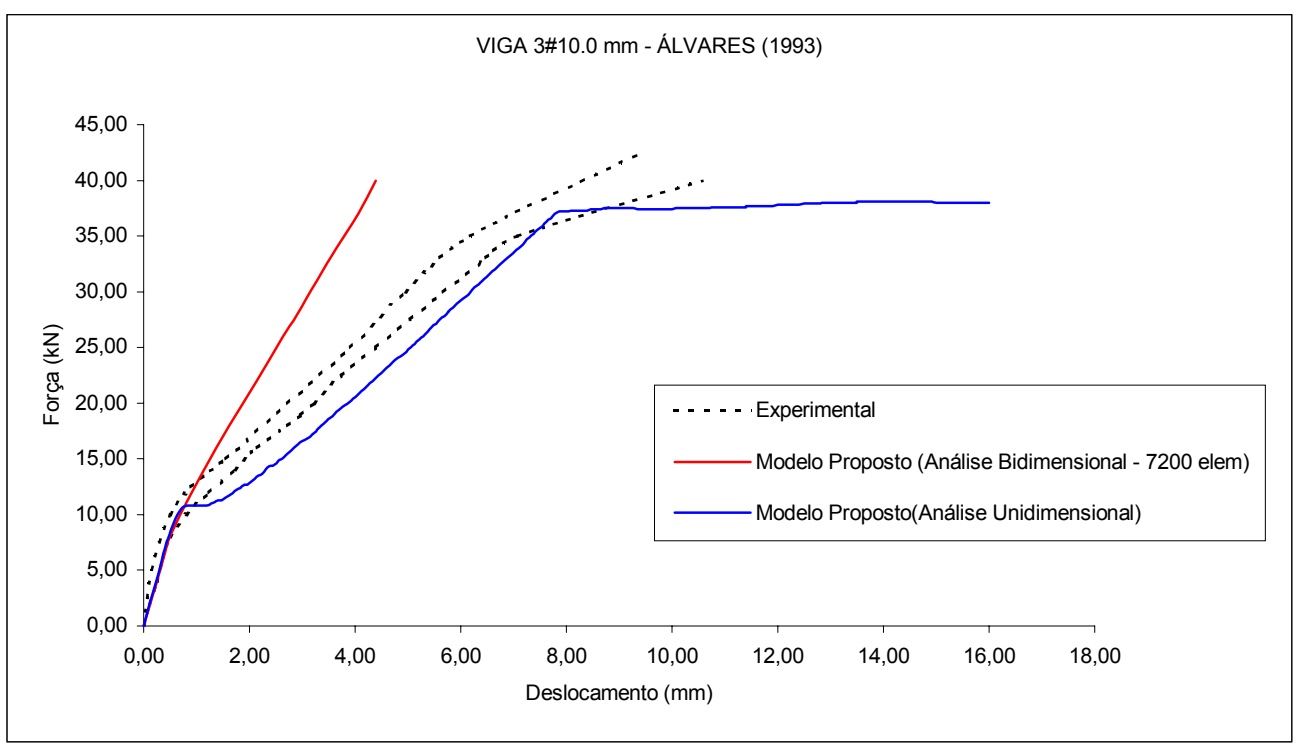

Figura 5.20 - Resultados numéricos (7200 elementos) - viga $3 \phi 10.0 \mathrm{~mm}$

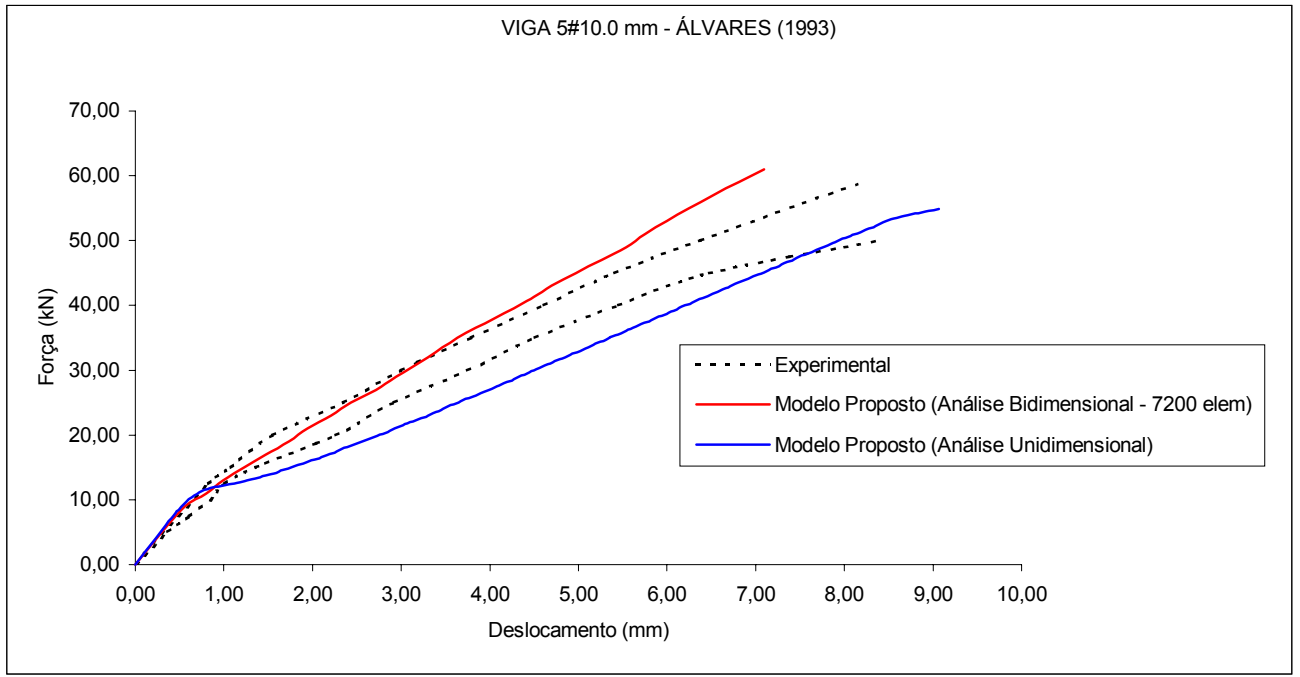

Figura 5.21 - Resultados numéricos (7200 elementos) - viga $5 \phi 10.0 \mathrm{~mm}$

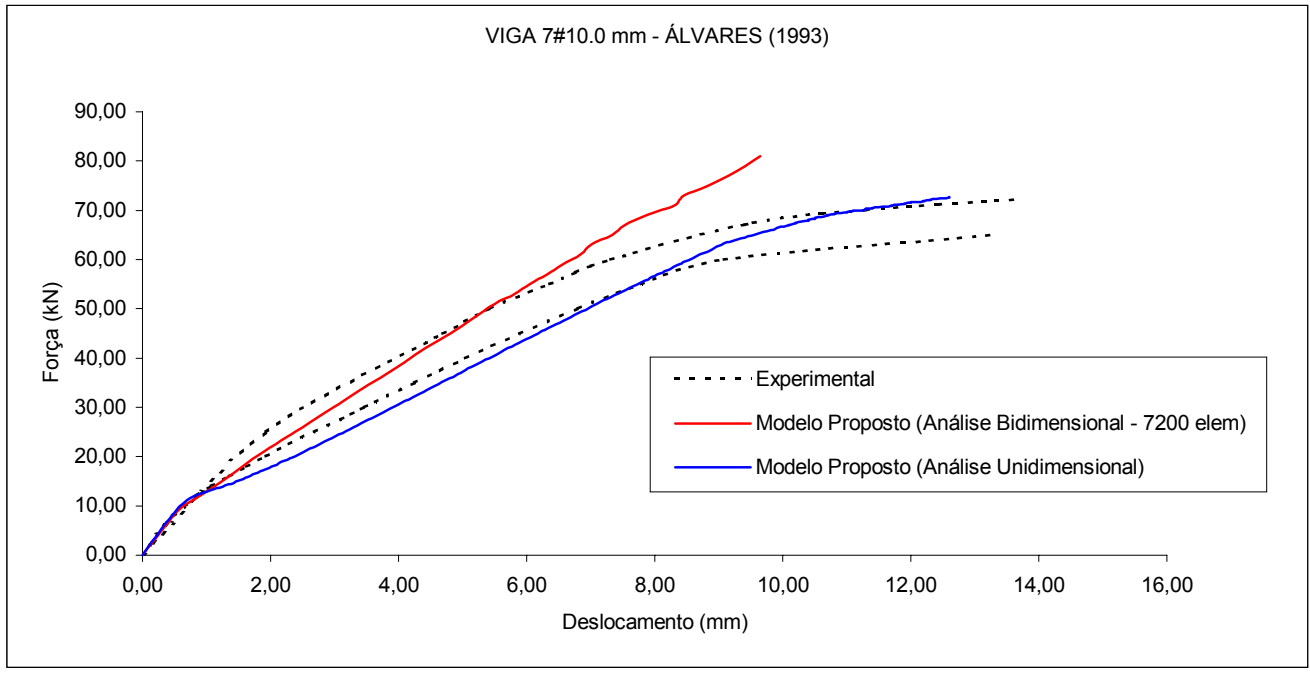

Figura 5.22 - Resultados numéricos (7200 elementos) - viga $7 \phi 10.0 \mathrm{~mm}$ 
Nas figuras a seguir são ilustradas as distribuições dos valores das variáveis de dano para a viga com alta taxa de armadura em algumas etapas de carregamento.
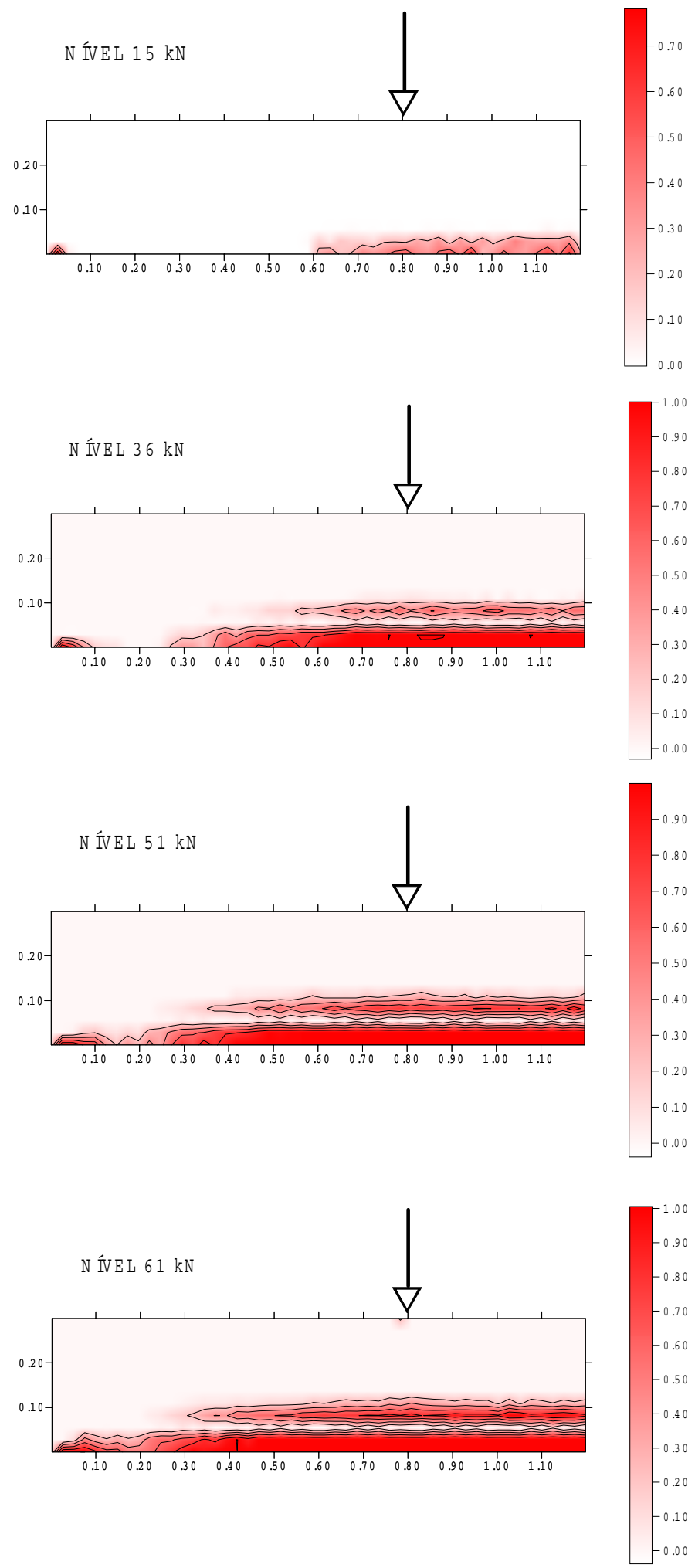

Figura 5.23 - Viga em concreto armado - viga $7 \phi 10.0 \mathrm{~mm}$ : distribuição de dano $\mathrm{D}_{1}$ 

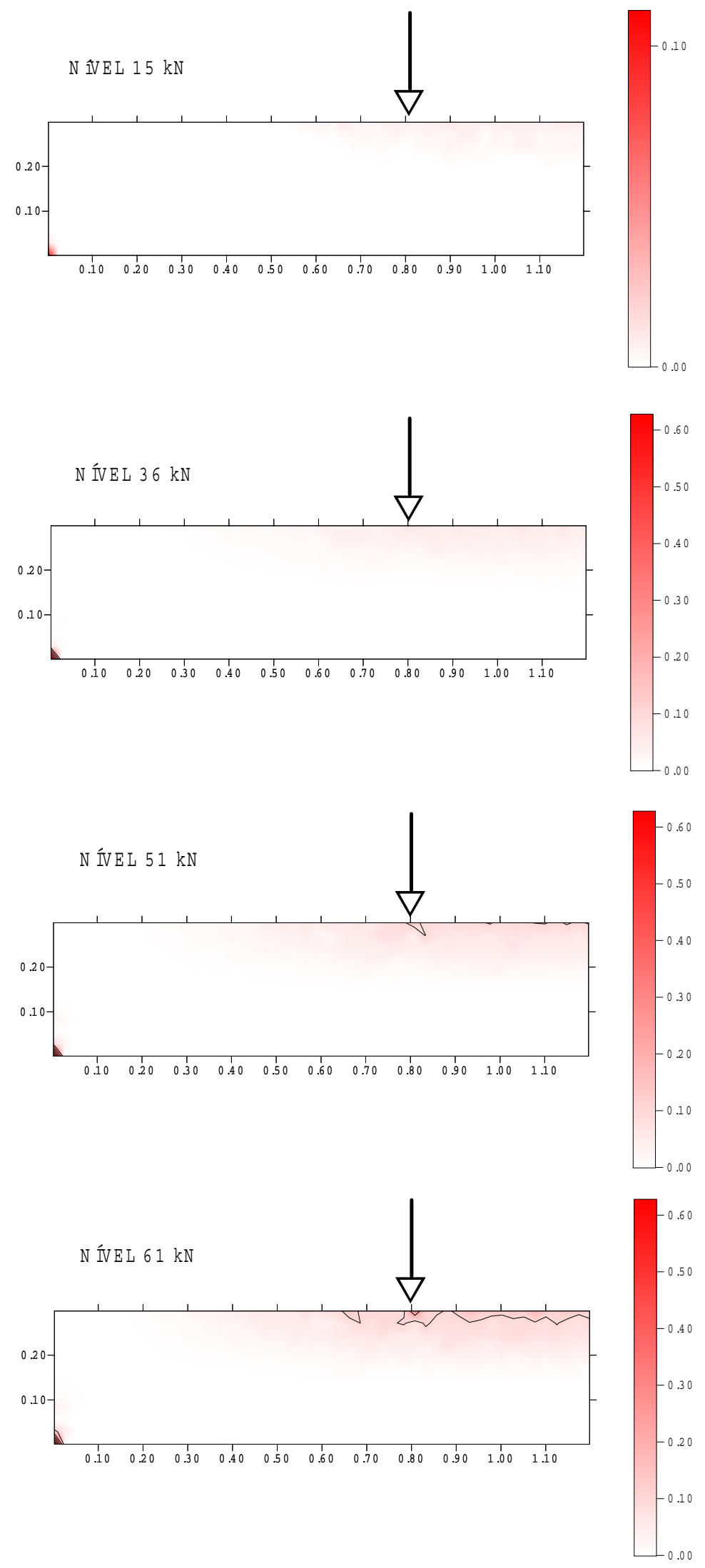

Figura 5.24 - Viga em concreto armado - viga $7 \phi 10.0 \mathrm{~mm}$ : distribuição de dano $\mathrm{D}_{2}$ 

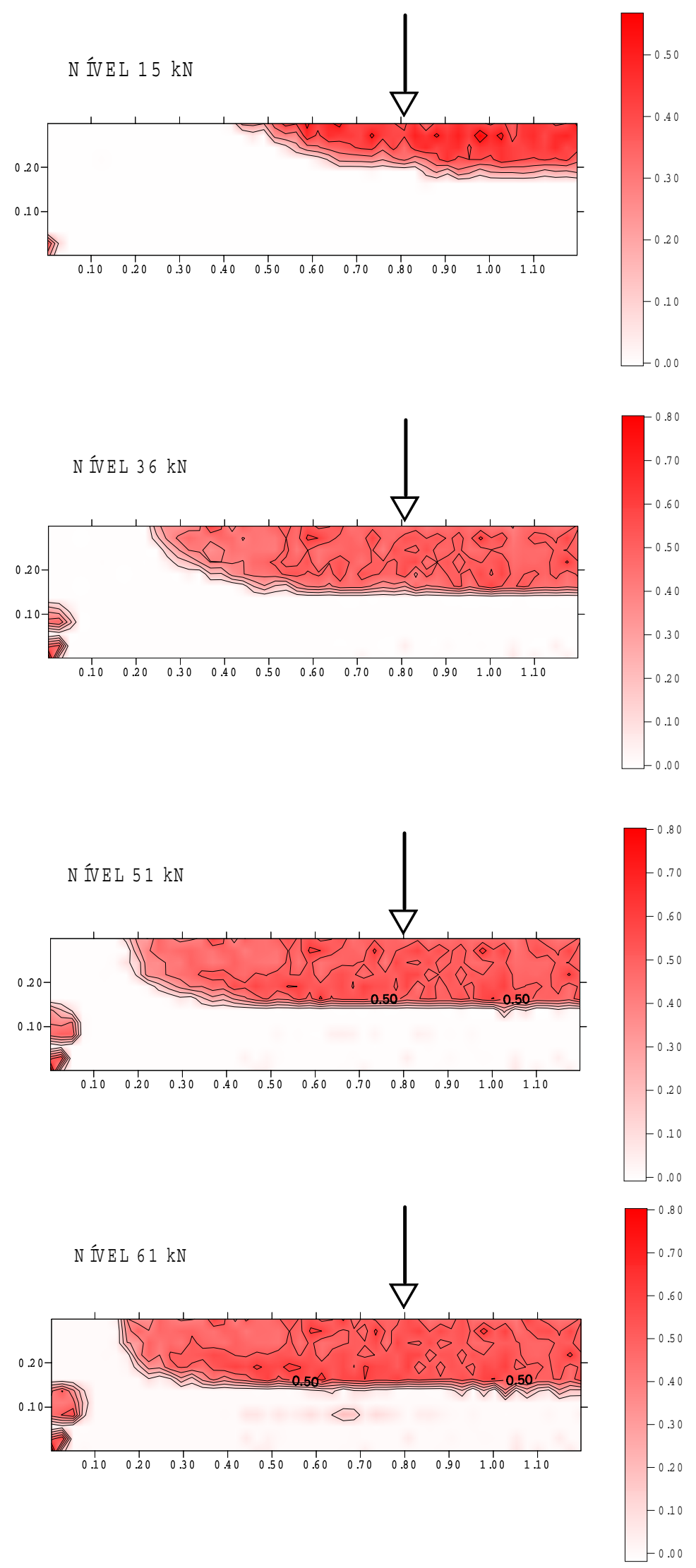

Figura 5.25 - Viga em concreto armado - viga $7 \phi 10.0 \mathrm{~mm}$ : distribuição de dano $\mathrm{D}_{3}$ 
Observa-se que para baixos níveis de carregamento a danificação em tração possui valores significativos na parte inferior da viga. Com o incremento da força aplicada a zona de danificação aumenta de baixo para cima e em direção à zona do apoio. Na parte superior da viga, a variável de dano $\mathrm{D}_{2}$ possui valores baixos em relação à variável $\mathrm{D}_{3}$ e ambas surgem também na zona do apoio em níveis próximos a força máxima.

Conclui-se, que o objetivo de validar o emprego da proposta de modelo na simulação do comportamento de estruturas em concreto armado foi alcançado. Entretanto, nas análises planas ficaram evidentes alguns problemas de ordem numérica principalmente no caso da viga pouco armada onde o panorama de fissuração é mais localizado [ver ÁLVARES (1993)]. Problemas similares surgidos na análise dessas vigas encontram-se em BARROS (2002). Pode-se concluir ainda que a fissuração localizada é claramente uma situação limitante do emprego do modelo proposto. 


\section{CONSIDERAÇÕES FINAIS, CONCLUSÕES E PERSPECTIVAS}

Este trabalho, de uma maneira geral, trata da formulação de modelos constitutivos para materiais elásticos danificados que possuem anisotropia e bimodularidade como características do seu comportamento. Por meio desta formulação e a partir de um estudo do comportamento mecânico do concreto, obtevese um modelo de dano para esse material, o qual leva em conta a isotropia transversal e o caráter bimodular induzidos pela danificação.

Inicialmente, num âmbito geral, foram comentados alguns fundamentos da Mecânica do Dano, entre eles os princípios de equivalência de deformação e de tensão, com destaque para o princípio de equivalência de energia que garante a simetria do tensor constitutivo. Com a aplicação dessas equivalências mostrou-se que é possível reduzir a ordem do tensor de dano. Em seguida relataram-se algumas evidências da danificação e a sua influência no comportamento físico do concreto. Abordaram-se tanto os mecanismos básicos de microfissuração, observando-se a microestrutura do material, quanto o comportamento macroscópico do concreto sob várias situações de solicitação. Com essa análise do comportamento físico procurouse justificar a formulação de modelos constitutivos para o concreto utilizando-se a hipótese de isotropia transversal induzida pelo dano.

No capítulo 2, apresentou-se a formulação de modelos constitutivos para meios elásticos anisótropos com diferentes comportamentos em tração e em compressão, desenvolvida por CURNIER, HE \& ZYSSET (1995). Tendo-se como base a formulação de Curnier, foi proposta neste trabalho uma extensão para os casos em que o meio sofre o processo de danificação. Dentro desse contexto, exemplificouse um caso de interesse para o trabalho: um meio elástico danificado com isotropia 
transversal e bimodularidade induzidas pelo dano. Por fim, apresentaram-se as possíveis formas do tensor constitutivo para diferentes modelos de dano isótropos e anisótropos dentro da visão da formulação aqui proposta.

Com base nos conceitos da Mecânica do Dano associados à proposta de extensão da formulação de Curnier, apresentou-se, no capítulo 3, uma proposta de modelo de dano para o concreto. Esse modelo é consistente com os princípios da termodinâmica e leva em conta a anisotropia e o caráter bimodular induzidos pelo dano. Na formulação do modelo, destacou-se a utilização do princípio de equivalência de energia para a obtenção de tensores constitutivos simétricos em tração e em compressão. Outros aspectos importantes do modelo são: a proposição de critérios para a ativação inicial e evolução do processo de danificação, de critérios para a caracterização dos estados predominantes de tração e de compressão e de regras para a identificação do plano local de isotropia transversal do material. Por fim, o capítulo 3 também apresenta uma proposta de incorporação das deformações plásticas devidas à danificação, para o caso de solicitação uniaxial.

O capítulo 4 abordou a identificação dos parâmetros inseridos nas leis de evolução das variáveis de dano. Nesse sentido, pesquisou-se a influência destes nas formas das curvas tensão-deformação. Algumas simulações numéricas de ensaios experimentais uniaxiais, biaxiais e triaxiais, contidos na literatura, foram apresentadas. As respostas obtidas foram consideradas bastante satisfatórias, contudo surgiram imprecisões na reprodução das deformações transversais na região póstensão de pico. Constatou-se que as imprecisões eram ocasionadas pela não consideração das deformações plásticas na formulação do modelo proposto. A consideração dessas deformações num caso uniaxial conduziu a uma melhora da resposta na região da tensão de pico da curva tensão-deformação, inclusive reproduzindo a inversão do sinal da deformação volumétrica no caso de compressão uniaxial.

Os resultados da aplicação do modelo em análises unidimensionais e bidimensionais de estruturas em concreto armado foram reunidos no capítulo 5, confrontando-se as respostas numéricas fornecidas pelo modelo proposto com as respostas experimentais colhidas na bibliografia consultada, além de respostas 
numéricas obtidas com os modelos de LA BORDERIE (1991) e de MAZARS (1984).

No caso das análises unidimensionais, verificou-se ótima concordância entre as respostas obtidas com o modelo proposto e as respostas experimentais de vigas em concreto armado ensaiadas por ÁLVARES (1993). No confronto com modelos isótropos de dano, propostos por La Borderie e Mazars, observou-se uma grande semelhança da resposta estrutural dentro dos limites do regime de serviço. Contudo, nesse regime, dada a simplificação dimensional, não ficaram evidenciadas as vantagens de se empregar um modelo com anisotropia induzida. Já nas proximidades do regime de ruptura, o modelo proposto, em razão de proporcionar um processo de danificação menos intenso, e por se tratar de um modelo formulado com a hipótese de equivalência de energia, apresentou uma resposta em que o concreto acabou por resistir aos esforços solicitantes de maneira mais eficiente. Os reflexos desse comportamento se manifestaram nos níveis de plastificação das armaduras, que aconteceram com valores de força aplicada mais próximos dos experimentais particularmente nos casos das vigas armadas com $5 \phi 10.0 \mathrm{~mm}$ e com $7 \phi 10.0 \mathrm{~mm}$, por se tratarem de vigas com uma fissuração mais difusa em relação à viga armada com $3 \phi 10.0 \mathrm{~mm}$. Pensa-se que estas conclusões podem servir de indício para o que acontece nas análises bidimensionais.

No caso do pórtico em concreto armado, apesar das limitações quanto à identificação paramétrica, o modelo proposto permitiu simular o comportamento da estrutura de maneira bastante satisfatória, sem requerer um refinamento mais apurado da malha. As evidências experimentais de início de fissuração e de plastificação das armaduras, relatadas em VECCHIO \& EMARA (1992), foram colhidas com suficiente precisão.

Nos casos de análises bidimensionais, pôde-se observar o bom resultado obtido pela aplicação do modelo na simulação do comportamento da viga ensaiada por PEREGO (1989). Essa viga tem a particularidade de apresentar uma elevada taxa de armadura, sendo a não-linearidade quase que exclusivamente decorrente do dano no concreto. Contudo, nos exemplos compostos por três séries de vigas, com diferentes taxas de armadura, surgiram problemas de ordem numérica inerentes à análise plana. Nos casos com menor taxa de armadura a fissuração passa a ser 
localizada após um certo nível de solicitação, limitando a aplicação de um modelo que pressupõe dano distribuído. Por fim, nos casos de maior taxa de armadura, constatou-se que pode ser importante a identificação das leis de evolução das variáveis de dano ligadas ao cisalhamento, recurso disponível pelo modelo mas que não foi explorado nos exemplos.

Pelas respostas apresentadas pelo modelo verificou-se a validação e a potencialidade do seu emprego na análise de estruturas em concreto armado.

\section{Conclusões Gerais e Perspectivas}

Quanto às contribuições do trabalho, deve-se destacar o estudo dos princípios de equivalência dentro da Mecânica do Dano, os quais permitem a redução da ordem do tensor de dano. Além disso, pode-se ressaltar a contribuição quanto à formulação de modelos constitutivos para meios elásticos danificados anisótropos com resposta bimodular e a obtenção, em caráter original, de um modelo de dano para o concreto.

Com relação ao campo da formulação matemática de modelos, a proposta de extensão da formulação de Curnier para a inclusão de processos de danificação é aplicável para materiais inicialmente isótropos ou anisótropos em algum grau e, ainda, para materiais que passam a apresentar anisotropia e aspecto bimodular induzidos pela danificação.

No âmbito dessa proposta, o modelo constitutivo para o concreto em princípio poderia incluir como hipótese a possibilidade do material apresentar ortotropia induzida pelo dano. Contudo, o custo da formulação teórica e principalmente da identificação paramétrica poderia tornar sua utilização prática inviável. Visto isso, optou-se pela hipótese de isotropia transversal induzida pelo dano, a qual, de fato, mostrou-se razoável para a simulação do comportamento do concreto, como ficou evidenciado nos exemplos de aplicação.

No campo estritamente numérico foram evidenciados alguns problemas, em parte inerentes ao tipo de discretização empregada na análise plana, tais como: travamento da resposta ('locking') devido à distorção exagerada de forma dos elementos, em particular da camada de elementos representativos do aço e irregularidade da forma da curva carga-deslocamento. Observou-se, também, uma 
tendência para resposta mais rígida em altos níveis de carga, provavelmente decorrente da imposição da aderência perfeita entre aço e concreto. Os problemas foram parcialmente resolvidos mediante uma discretização mais refinada, porém não foram todos contornados, uma vez que parte deles decorre de condições não necessariamente ligadas à discretização, como à localização da danificação nas vigas com baixa taxa de armadura. Apesar das dificuldades numéricas, considera-se que o objetivo de validação do emprego do modelo proposto na análise de estruturas em concreto armado foi plenamente alcançado.

O modelo potencialmente pode ainda ser empregado na verificação do comportamento de peças sob fadiga e na análise de estruturas sujeitas a cargas provenientes de sismos. Vale ressaltar, além disso, que a formulação dada ao modelo permite adaptá-lo, com a conveniente identificação de parâmetros, para a simulação do comportamento de materiais compostos que apresentam comportamento bimodular e isotropia transversal induzidas pelo processo de danificação. De um modo geral, como já comentado, a formulação para meios anisótropos proposta no capítulo 2 tem um caráter geral, de forma que este trabalho abre um grande leque para o desenvolvimento de modelos que visem simular o comportamento de meios onde a danificação é o principal fenômeno envolvido.

Há, por outro lado, contribuições estritamente ligadas à álgebra tensorial, reunidas no apêndice. Entre elas destaca-se a obtenção da forma matricial dos tensores de quarta ordem envolvidos na formulação. Essa forma tem interesse para a implementação do modelo em código de cálculo.

Obviamente, do trabalho realizado resultam algumas perspectivas claras para a sua continuação. De imediato, pode-se sugerir a consideração de deformações plásticas nas simulações que permitem a utilização da versão uniaxial do modelo proposto. A partir daí, pode-se verificar a potencialidade de sua aplicação em análises de estruturas lineares em concreto armado submetidas a situações de carga e descarga, contemplando, inclusive, a inversão de sinal da solicitação. Na seqüência, pode-se estudar a incorporação das deformações plásticas na formulação do modelo com vistas às situações de solicitações gerais.

Outra sugestão para a continuidade das pesquisas seria a realização de ensaios em elementos de concreto submetidos a solicitações que permitissem a identificação 
de leis de evolução das variáveis de dano ligadas ao cisalhamento. Nesse contexto, propõe-se a extensão da investigação para a interação entre o concreto e o aço.

Ainda no âmbito da formulação teórica, pode-se sugerir a proposição de um modelo com ortotropia induzida pela danificação, o que não foi feito neste trabalho pelos motivos já explicados, e averiguar as possíveis vantagens dessa formulação.

No campo da transição dano para fratura, pode-se pesquisar uma versão do modelo com localização de deformações. Este é um tema interessante para a formulação teórica, pois a localização de deformações em modelos que consideram a anisotropia do material pode constituir-se num vasto campo de pesquisa.

Dentro do escopo numérico, sugere-se a implementação do modelo proposto num código de cálculo que permita a análise tridimensional de estruturas de concreto, para investigar, de modo mais completo, as vantagens em relação aos modelos isótropos.

Ainda dentro do campo numérico, sugere-se a implementação do modelo proposto num código de cálculo que empregue formas não-convencionais do método dos elementos finitos, como o Método dos Elementos Finitos Generalizados, já em franco desenvolvimento no Departamento de Engenharia de Estruturas. Tal método pode apresentar vantagens particularmente quanto à definição de zonas de localização e evolução do dano.

Os temas citados e outros de igual importância constituem, sem sombra de dúvidas, um amplo campo de pesquisa a ser explorado. 


\section{REFERÊNCIAS BIBLIOGRÁFICAS}

ÁLVARES, M. S. (1993). Estudo de um modelo de dano para o concreto: formulação, identificação paramétrica e aplicação com emprego do método dos elementos finitos. São Carlos. Dissertação (Mestrado) - Escola de Engenharia de São Carlos, Universidade de São Paulo.

BARROS, F. B. (2002). Métodos sem malha e método dos elementos finitos generalizados em análise não-linear de estruturas. São Carlos. Tese (Doutorado) Escola de Engenharia de São Carlos, Universidade de São Paulo.

BAZANT, Z. P.; PIJAUDIER-CABOT, G. (1989). Measurement of characteristic length of nonlocal continuum. Journal of Engineering Mechanics, ASCE, pp. 755-767.

BERTACCHI, P.; BELOTTI, R. (1972). Experimental research on deformation and failure of concrete under triaxial loads. Proceedings International Symposium RILEM, Cannes, pp. 37-52.

BERTHAUD, Y. (1991). Mesures et modelisations de l'endommagement des materiaux. Laboratoire de Mecanique et Technologie, Cachan, France.

BETTEN, J. (1983). Tenseus d'endommagement en mécanique des milieux continus. Journal de Mécanique Théorique et Appliquée, vol. 2, n. 1, pp. 13-32.

BITTENCOURT, T. N. (1999). Fraturamento do concreto estrutural : aspectos teóricos, computacionais e experimentais, e suas aplicações. São Paulo. Tese de Livre Docência - Escola Politécnica da Universidade de São Paulo.

Bittencourt, T. N.; BORGES, J. U. A.; PRAdO, E. P.; GUimarÃes, A. E. P.; SANTOS, A. C.; FERREIRA, L. E. T. (2000). Estudo experimental do 
fraturamento do concreto estrutural por meio de corpos-de-prova cilíndricos. Escola Politécnica da Universidade de São Paulo, 31p.

BUYUKOZTURK, O.; NILSON, A. H.; SLATE, F. O. (1972). Deformation and fracture of paritulate composite. J. Eng. Mech., ASCE, v. 98, n. EM3, Jun.

CARPINTERI, A. (1986). Mechanics damage and crack growth in concrete. Dordrecht : Martinus Nijhoff, 234p.

CAUVIN, A. (1997). Damage mechanics:basic variables in continuum theories. Doctoral Dissertation, Columbia University.

CAUVIN, A.; TESTA, R. B. (1999). Elastoplastic materials with isotropic damage. International Journal of Solids and Structures, vol. 36, pp. 727-746.

CAUVIN, A.; TESTA, R. B. (1999). Damage mechanics: basic variables in continuum theories. International Journal of Solids and Structures, vol. 36, pp. 747-761.

CAZACU, O.; CRISTESCU, N. D.; SHAO, J. F. ; HENRY, J. P. (1998). A new anisotropic failure criterion for transversely isotropic solids. Mechanics of Cohesive-Frictional Materials, vol. 3, pp. 89-103.

CHABOCHE, J. L. (1988). Continuum damage mechanics: part I - general concepts. Journal of Applied Mechanics, vol. 55, pp. 59-64.

CHEN, W. F. (1982). Plasticity in reinforced concrete. Mc Graw Hill.

CHOW, C. L.; WANG, J. (1987). An anisotropic theory of elasticity for continuum damage mechanics. International Journal of Fracture, vol. 33, pp. 3-16.

COMI, C. (2000). A nonlocal damage model with permanent strains for quasi-brittle materials. Continuous Damage and Fracture. Ed.: Ahmed Benallal, pp. 221-232. 
COMI, C.; BERTHAUD, Y.; BILLARDON, R. (1995). On localization in ductilebrittle materials under compressive loadings. European Journal of Mechanics, A/Solids, vol. 14, n. 1, pp. 19-43.

COMI, C.; PEREGO, U. (2000). A bi-dissipative damage model for concrete with applications to dam engineering. European Congress on Computational Methods in Applied Sciencs and Engineering - ECOMAS 2000.

CORDEBOIS, J. P.; SIDOROFF, F. (1979). Damage induced elastic anisotropy. Colloque Euromech 115, Villars de Lans.

CURNIER, A.; HE, Q.; ZYSSET, P. (1995). Conewise linear elastic materials. Journal of Elasticity, vol. 37, pp. 1-38.

DEL PIERO, G. P. (1979). Some properties of the set of fourth-order tensors, with application to elasticity. Journal of Elasticity, vol. 9, pp. 245-261.

DIAZ, S.; HILSDORF, H. K. (1973). Fracture mechanisms of concrete under compressive loads. Cement and Concrete Research, vol. 3, pp. 363-388.

FICHANT, S.; PIJAUDIER-CABOT, G.; LA BORDERIE, C. (1997). Continuum damage modelling: approximation of crack induced anisotropy. Mechanics Research Communications, vol. 24, n. 2, pp. 109-114.

FICHANT, S.; LA BORDERIE, C.; PIJAUDIER-CABOT, G. (1999). Isotropic and anisotropic descriptions of damage in concrete structures. Mechanics of Cohesive-Frictional Materials, vol. 4, pp. 339-359.

FIGUERAS, J. A. (1983). Ultimate load analysis of anisotropic and reinforced concrete plates and shells. PhD Thesis, University of Wales, Department of Civil Engineering. 
FOKWA, D.; BERTHAUD, Y. (1993). Heterogeneous materials: experimental análisis of localization and the influence size of the heterogeneities on the behaviour in tension. Materials and Structures, vol.26, pp. 136-143.

GLOMB, J. A.; PATAS, P. J. (1972). Investigations concerning the behaviour of lightweight concrete in a state of triaxial pressure. Proceedings International Symposium RILEM Cannes, pp. 71-78.

GOPALARATNAM, V. S.; SHAH, S. P. (1985). Softening response of plain concrete in direct tension. ACI Journal, may-june, pp. 310-323.

GOVINDJEE, S.; KAY, G. J.; SIMO, J. C. (1995). Anisotropic modelling and numerical simulation of brittle damage in concrete. International Journal for Numerical Methods in Engineering, vol.35, pp. 3611-3633

GUELLO, G. A. (2002). Simulação computacional de estruturas de concreto por meio da mecânica do dano. São Paulo. Dissertação (Mestrado) - Escola Politécnica da Universidade de São Paulo.

HALM, D.; DRAGON, A. (1998). An anisotropic model of damage and frictional sliding for brittle materials. European Journal of Mechanics, A/Solids, n. 3, pp. 439-460.

HAND, F. R.; PECKNOLD, D. A.; SCHNOBRICH, W. C. (1973). Non linear layered analysis of reinforced concrete. Plates and shells. Journal of the Structural Division, vol. 99, n. ST7, july, pp. 1491-1505.

HE, Q.; CURNIER, A. (1995). A more fundamental approach to damage elastic stress-strain relations. International Journal of Solids and Structures, vol. 32, pp. $1433-1457$. 
HEILMANN, H. G.; HILSDORF, H. H.; FINSTERWALDER, K. (1969). Festigkeit und verformung von beton unter zugspannungen. Deutschet Ausschuss für Stahlbeton, Heft 203,Berlin.

HILLEBORG, A.; PETERSSON, P. E. (1981). Fracture mechanical calculations, test methods and results and similar materials.Advances in Fracture Research (ICF 5 - Cannes), vol. 4, pp. 1515 - 1522.

HOENIG, A. (1979). Elastic moduli of a non-randomly cracked body. International Journal of Solids and Structures, vol. 15, pp. 137-154.

JONES, R. M. (1977). Stress-strain relations for materials with different moduli in tension and compression. AIAA Journal, vol. 15, pp. 16-23.

JU, J. W. (1989). On energy-based coupled elastoplastic damage theories: constitutive modeling and computational aspects. International Journal of Solids and Structures, vol. 25, n. 7, pp. 803-833.

JU, J. W. (1990). Isotropic and anisotropic damage variables in continuum damage mechanics. Journal of Engineering Mechanics, ASCE, vol 116, n. 12, pp. 27642770.

KACHANOV, L. M. (1958). Time of the rupture process of non-linear solid mechanics. Otd. Tech. Nauk., v. 8, pp. 28-31.

KADLECEK, V.; SPETLA, Z. (1967). Effect of size and shape on test specimens on the direct tensile strength of concrete. Bulletin RILEM n. 36, September, pp. 175-184.

KRAJCINOVIC, D (1985). Continuous damage mechanics revisited: basic concepts and definitions. Journal of Applied Mechanics, v. 52, pp. 829-834. 
KUHL, E.; RAMM, E.; de BORST, R. (2000). An anisotropic gradiente damage model for quasi-brittle materials. Computational Methods Applied in Mechanics Engineering, vol. 183, pp. 87-103.

KUPFER, H.; HILSDORF, H. K.; RUSCH, H. (1969). Behavior of concrete under biaxial stresses. ACI Journal, vol. 66, pp. 656- 666.

LABADI, Y.; HANNACHI, N. E. (1997). Finite element analysis of anisotropic damage mechanics problems. Computational Plasticity, CIMNE, Barcelona, pp. 1102-1107.

LA BORDERIE, C. (1991). Phenomenes unilateraux dans un materiau endommageable: modelisation et application a l'analyse de structures en beton. These de Doctorat de l'universite Paris.

LA BORDERIE, C.; MAZARS, J.; PIJAUDIER-CABOT, G (1991). Response of plain and reinforced concrete structures iunder cyclic loadings. Cachan, France, Laboratoire de Mécanique et Technologie, Rapport Interne, n. 123.

LA BORDERIE, C.; MAZARS, J.; PIJAUDIER-CABOT, G (1992). Computational modelling of concrete and reinforced concrete based on damage mechanics. In: Computational Plasticity, eds.: Owen, D. R. J., Onãte, E., Hinton, E., Barcelona.

LADEVÈZE, P. (1983). Sur une théorie de l'endommagement anisotrope. Cachan, France, Laboratoire de Mécanique et Technologie, Rapport Interne, n. 34.

LADEVÈZE, P.; LETOMBE, S. (2000). Recent Advances an anisotropic damage theory including unilateral effects. Continuous Damage and Fracture. Ed.: Ahmed Benallal, pp. 81-87.

LEMAITRE, J. (1996). A course on damage mechanics. Springer Verlag. 
LEMAITRE, J.; CHABOCHE, J. L. (1990). Mechanics of solid materials. Cambridge University Press.

LEMAITRE, J.; DESMORAT, R.; SAUZAY, M. (2000). Anisotropic damage law of evolution. European Journal of Mechanics, A/Solids, vol. 19, pp. 187-208.

L'HERMITE, R. (1973). Influence de la dimension absolue sur la résistance à la flexion. Annales de l'ITBTP, n. $309-310$, pp. $39-41$.

LIU, I. (1982). On representations of anisotropic invariants. International Journal of Engineering Science, vol. 20, n. 10, pp. 1099-1109.

MAZARS, J. (1984). Application de la mécanique de l'endommagement au comportement non lineaire et à la rupture du béton de structure. Thése de Doctorat d'État, Université Paris 6.

MAZARS, J.; BERTHAUD, Y.; RAMTANI, S. (1990). The unilateral behavior of damage concrete. Engineering Fracture Mechanics, v. 35, n. 4/5, pp. 629-635.

MAZARS, J.; RAMTANI, S.; BERTHAUD, Y. (1988). An experimental procedure to delocalize tensile failure and identify the unilateral effect of distributed damage. Cracking and Damage - Strain Localization and Size Effect.

MESCHKE, G.; LACKNER, R.; MANG, H. A. (1998). An anisotropic elastoplasticdamage model for plain concrete. International Journal for Numerical Methods in Engineering, vol. 42, pp. 703-727.

MURAKAMI, S. (1987). Anisotropic aspects of material damage and application of continuum damage mechanics. Continuum Damage Mechanics: Theory and Applications. C. I. S. M. Courses and Lectures n. 295, eds: Krajcinovic, D. and Lemaitre, J. 
MURAKAMI, S; KAMIYA, K. (1997). Constitutive and damage evolution equations of elastic-brittle materials based on irreversible thermodynamics. Internationational Journal of mechanics Science, vol. 39, n. 4, pp. 473-486.

ORTIZ, M. (1985). A constitutive theory for the inelastic behavior of concrete. Mechanics of Materials, vol. 4, pp. 67-93.

ORTIZ, M. (1987). An analitical study of the localized failure modes of concrete. Mechanics of Materials, vol. 6, pp. 159-174.

OWEN, D. R. J.; HINTON, E. (1980). Finite elements in plasticity: theory and practice. Pineridge Press, Swansea, U. K..

PAPA, E.; TALIERCIO (1996). Anisotropic damage model for the multiaxial static and fatigue behaviour of plain concrete. Engineering Fracture Mechanics, vol. 55 , n. 2, pp. 163-179.

PEREGO, M. (1989). Danneggiamento dei materiali lapidei: leggi constitutive, analisis per elementi finiti ed applicazioni. Tesi di Laurea, Politecnico di Milano.

PIETRUSZCZAK, S.; MROZ, Z. (2001). On failure criteria for anisotropic cohesivefrictional materials. International Journal for Numerical and Analytical Methods in Geomechanics, vol. 25, pp. 509-524.

PITUBA, J. J. C. (1998). Estudo e aplicação de modelos constitutivos para o concreto fundamentados na mecânica do dano contínuo. São Carlos. Dissertação (Mestrado) - Escola de Engenharia de São Carlos, Universidade de São Paulo.

PITUBA, J. J. C.; PROENÇA, S. P. B.; ÁlVARES, M. S. (1999). Estudo do Desempenho de Modelos de Dano para Estruturas Reticulares em Concreto Armado [CD-ROM]. In: XX CILAMCE - Congresso Íbero Latino-Americano de Métodos Computacionais em Engenharia, São Paulo - SP. 
PITUBA, J. J. C.; PROENÇA, S. P. B. (2002). Um modelo constitutivo para o concreto considerando-se anisotropia e rigidez bimodular induzidas pelo dano. XXX Jornadas Sul-Americanas de Engenharia Estrutural, Brasília, DF - Brasil.

PROENÇA, S. P. B. (1988). Sobre modelos matemáticos do comportamento nãolinear do concreto: análise crítica e contribuições. Tese de Doutorado, EESC/USP.

RABOTNOV, Y. N. (1969). Creep problems in structural members. Amsterdam, North-Holland.

RAMTANI, S. (1990). Contribution à la modélisation du comportement multiaxial du béton endommagé avec discription du caractère unilatéral. Thèse de Doctorat de l’Université Paris 6.

RAMTANI, S.; BERTHAUD, Y.; MAZARS, J. (1992). Orthotropic behavior of concrete with directional aspects: modeling and experiments. Nuclear Engineering Design, vol. 133, pp. 97-111.

RIZZI, E. (1995). Sulla localizzazione delle deformazioni in materiali e strutture. Tesi di Dottorato di Ricerca in Ingegneria delle Strutture, Politecnico di Milano.

SPENCER, A. J. M. (1980). Continuum mechanics. Longman Scientific \& Technical.

STROEVEN, P. (1979). Geometric probability approach to the examination of microcracking in plain concrete. Journal of Materials Science, vol. 14, pp. 11411151.

TERRIEN, m. (1980). Emission acoustique et comportement mécanique postcritique d'un béton sollicité en traction. Bulletin de Liaison Ponts et Chaussées, n. 105 , pp. $65-72$. 
VAN MIER, G. M. (1984). Strain-softening of concrete under multiaxial loading conditions. PhD Thesis, Eindhoven Tech. Univ..

VECCHIO, F. J.; EMARA, M. B. (1992). Shear deformations in reinforced concrete frames. ACI Structural Journal, vol. 89, n. 1, pp. 46-56.

WELEMANE, H.; CORMERY, F. (2001). Some remarks on the damage unilateral effect modeling for microcracked materials. XV Congrès Français de Mécanique, Nancy.

WILlAM, K.; HURLBUT, B.; STURE, S. (1985). Experimental and constitutive aspects of concrete failure. Finite Element Analysis of Reinforced Concrete Structures. Tokyo, Japan.

WILLAM, K.; STANKOWSKI, T.; STURE, S. (1988). Theory and basic concepts for modelling concrete behavior. Contribution to the $26^{\text {th }}$ CEB Plenary Session, Dubrovnik. 


\section{APÊNDICE: RELAÇÕES MATEMÁTICAS DE INTERESSE}

\section{INTRODUÇÃO}

Neste apêndice são apresentados os tensores de quarta ordem utilizados durante toda a formulação contida neste trabalho.

Inicialmente são abordados os tensores isótropos de quarta ordem, e suas propriedades, que servem de base para a obtenção de outros tensores. Em seguida, trata-se do tensor $\mathbf{A}$, dependente da definição do plano local de isotropia transversal. Também apresentam-se as operações tensoriais que formam os tensores de quarta ordem necessários para a proposição do modelo.

Por fim, explicitam-se as formas dos tensores de quarta ordem em notação matricial

\section{TENSORES ISÓTROPOS DE QUARTA ORDEM}

Existem três tensores isótropos de quarta ordem linearmente independentes [SPENCER (1980)]:

$$
\begin{aligned}
& \mathbf{I} \otimes \mathbf{I}=\delta_{\mathrm{ij}} \delta_{\mathrm{kl}}\left(\mathrm{e}_{\mathrm{i}} \otimes \mathrm{e}_{\mathrm{j}} \otimes \mathrm{e}_{\mathrm{k}} \otimes \mathrm{e}_{\mathrm{l}}\right) \\
& \mathbf{I} \otimes \mathbf{I}=\delta_{\mathrm{ik}} \delta_{\mathrm{jl}}\left(\mathrm{e}_{\mathrm{i}} \otimes \mathrm{e}_{\mathrm{j}} \otimes \mathrm{e}_{\mathrm{k}} \otimes \mathrm{e}_{1}\right) \\
& \mathbf{I} \otimes \mathbf{I}=\delta_{\mathrm{il}} \delta_{\mathrm{jk}}\left(\mathrm{e}_{\mathrm{i}} \otimes \mathrm{e}_{\mathrm{j}} \otimes \mathrm{e}_{\mathrm{k}} \otimes \mathrm{e}_{1}\right)
\end{aligned}
$$


onde $\mathbf{I}\left[\mathbf{I}=\delta_{\mathrm{ij}}\left(\mathrm{e}_{\mathrm{i}} \otimes \mathrm{e}_{\mathrm{j}}\right)\right]$ é o tensor isótropo identidade de segunda ordem, nomenclatura derivada do fato de que suas componentes não mudam seja qual for o sistema de coordenadas.

Qualquer outro tensor de quarta ordem é combinação linear dos anteriores. Sendo assim, um particular tensor de interesse decorrente das relações (1), (2) e (3) é dado por:

$$
\underline{\mathbf{I}} \underline{\overline{\mathbf{I}}}=\frac{1}{2}[(\mathbf{I} \otimes \mathbf{I})+((\overline{\mathbf{I}} \otimes \mathbf{I})]
$$

No que segue, são apresentadas propriedades características dos tensores isótropos de quarta ordem explicitadas nas relações de (1) a (4). Nesse sentido, considere-se um tensor de segunda ordem $\mathbf{S}$ que sofre a aplicação dos tensores isótropos de quarta ordem:

$$
\begin{aligned}
(\mathbf{I} \otimes \mathbf{I}) \mathbf{S} & =\delta_{\mathrm{ij}} \delta_{\mathrm{kl}} \mathrm{S}_{\mathrm{mn}}\left(\mathrm{e}_{\mathrm{i}} \otimes \mathrm{e}_{\mathrm{j}} \otimes \mathrm{e}_{\mathrm{k}} \otimes \mathrm{e}_{1}\right)\left(\mathrm{e}_{\mathrm{m}} \otimes \mathrm{e}_{\mathrm{n}}\right) \\
& =\delta_{\mathrm{ij}} \delta_{\mathrm{kl}} \mathrm{S}_{\mathrm{mn}} \delta_{\mathrm{km}} \delta_{\ln }\left(\mathrm{e}_{\mathrm{i}} \otimes \mathrm{e}_{\mathrm{j}}\right) \\
& =\delta_{\mathrm{ij}} \delta_{\mathrm{kl}} \mathrm{S}_{\mathrm{kl}}\left(\mathrm{e}_{\mathrm{i}} \otimes \mathrm{e}_{\mathrm{j}}\right) \\
& =\delta_{\mathrm{ij}} \mathrm{S}_{\mathrm{kk}}\left(\mathrm{e}_{\mathrm{i}} \otimes \mathrm{e}_{\mathrm{j}}\right) \\
& =(\operatorname{tr} \mathbf{S}) \mathbf{I}
\end{aligned}
$$

Nota-se que:

$\mathbf{S} . \mathbf{I}=\mathrm{S}_{\mathrm{ij}} \delta_{\mathrm{kl}}\left(\mathrm{e}_{\mathrm{i}} \otimes \mathrm{e}_{\mathrm{j}}\right) \cdot\left(\mathrm{e}_{\mathrm{k}} \otimes \mathrm{e}_{1}\right)=\mathrm{S}_{\mathrm{ij}} \delta_{\mathrm{kl}} \delta_{\mathrm{ik}} \delta_{\mathrm{jl}}=\mathrm{S}_{\mathrm{ij}} \delta_{\mathrm{ij}}=\mathrm{S}_{\mathrm{ii}}=\operatorname{tr} \mathbf{S}$

Portanto, tem-se que a aplicação em questão gera um tensor esférico:

$$
\begin{aligned}
& (\mathbf{I} \otimes \mathbf{I}) \mathbf{S}=(\operatorname{tr} \mathbf{S}) \mathbf{I}=(\mathbf{S} . \mathbf{I}) \mathbf{I} \\
& (\underline{\mathbf{I}} \underline{\mathbf{I}}) \mathbf{S}=\delta_{\mathrm{ik}} \delta_{\mathrm{jl}} \mathrm{S}_{\mathrm{mn}}\left(\mathrm{e}_{\mathrm{i}} \otimes \mathrm{e}_{\mathrm{j}} \otimes \mathrm{e}_{\mathrm{k}} \otimes \mathrm{e}_{1}\right)\left(\mathrm{e}_{\mathrm{m}} \otimes \mathrm{e}_{\mathrm{n}}\right)
\end{aligned}
$$




$$
\begin{aligned}
& =\delta_{\mathrm{ik}} \delta_{\mathrm{jl}} \mathrm{S}_{\mathrm{mn}} \delta_{\mathrm{km}} \delta_{\ln }\left(\mathrm{e}_{\mathrm{i}} \otimes \mathrm{e}_{\mathrm{j}}\right) \\
& =\delta_{\mathrm{ik}} \delta_{\mathrm{jl}} \mathrm{S}_{\mathrm{kl}}\left(\mathrm{e}_{\mathrm{i}} \otimes \mathrm{e}_{\mathrm{j}}\right) \\
& =\mathrm{S}_{\mathrm{ij}}\left(\mathrm{e}_{\mathrm{i}} \otimes \mathrm{e}_{\mathrm{j}}\right) \\
& =\mathbf{S}
\end{aligned}
$$

Portanto, $(\mathbf{I} \otimes \mathbf{I})$ é o tensor identidade de quarta ordem.

$$
\begin{aligned}
(\mathbf{I} \otimes \mathbf{I}) \mathbf{S} & =\delta_{\mathrm{il}} \delta_{\mathrm{jk}} \mathrm{S}_{\mathrm{mn}}\left(\mathrm{e}_{\mathrm{i}} \otimes \mathrm{e}_{\mathrm{j}} \otimes \mathrm{e}_{\mathrm{k}} \otimes \mathrm{e}_{1}\right)\left(\mathrm{e}_{\mathrm{m}} \otimes \mathrm{e}_{\mathrm{n}}\right) \\
& =\delta_{\mathrm{il}} \delta_{\mathrm{jk}} \mathrm{S}_{\mathrm{mn}} \delta_{\mathrm{km}} \delta_{\ln }\left(\mathrm{e}_{\mathrm{i}} \otimes \mathrm{e}_{\mathrm{j}}\right) \\
& =\delta_{\mathrm{il}} \delta_{\mathrm{jk}} \mathrm{S}_{\mathrm{kl}}\left(\mathrm{e}_{\mathrm{i}} \otimes \mathrm{e}_{\mathrm{j}}\right) \\
& =\mathrm{S}_{\mathrm{ji}}\left(\mathrm{e}_{\mathrm{i}} \otimes \mathrm{e}_{\mathrm{j}}\right) \\
& =\mathbf{S}^{\mathbf{T}}
\end{aligned}
$$

Portanto, o tensor ( $\mathbf{I} \bar{\otimes} \mathbf{I})$ opera a transposição do tensor $\mathbf{S}$.

$$
\begin{aligned}
(\underline{\mathbf{I} \otimes} \underline{\mathbf{I}}) \mathbf{S} & =\frac{1}{2}\left[\delta_{\mathrm{ik}} \delta_{\mathrm{jl}}+\delta_{\mathrm{il}} \delta_{\mathrm{jk}}\right] \mathrm{S}_{\mathrm{mn}}\left(\mathrm{e}_{\mathrm{i}} \otimes \mathrm{e}_{\mathrm{j}} \otimes \mathrm{e}_{\mathrm{k}} \otimes \mathrm{e}_{1}\right)\left(\mathrm{e}_{\mathrm{m}} \otimes \mathrm{e}_{\mathrm{n}}\right) \\
& =\frac{1}{2}\left[\delta_{\mathrm{ik}} \delta_{\mathrm{jl}}+\delta_{\mathrm{il}} \delta_{\mathrm{jk}}\right] \mathrm{S}_{\mathrm{mn}} \delta_{\mathrm{km}} \delta_{\mathrm{ln}}\left(\mathrm{e}_{\mathrm{i}} \otimes \mathrm{e}_{\mathrm{j}}\right) \\
& =\frac{1}{2}\left[\delta_{\mathrm{ik}} \delta_{\mathrm{jl}}+\delta_{\mathrm{il}} \delta_{\mathrm{jk}}\right] \mathrm{s}_{\mathrm{kl}}\left(\mathrm{e}_{\mathrm{i}} \otimes \mathrm{e}_{\mathrm{j}}\right) \\
& =\frac{1}{2}\left[\delta_{\mathrm{ik}} \delta_{\mathrm{jl}} \mathrm{S}_{\mathrm{k} 1}+\delta_{\mathrm{il}} \delta_{\mathrm{jk}} \mathrm{S}_{\mathrm{k} 1}\right]\left(\mathrm{e}_{\mathrm{i}} \otimes \mathrm{e}_{\mathrm{j}}\right) \\
& =\frac{1}{2}\left[\mathrm{~S}_{\mathrm{ij}}+\mathrm{S}_{\mathrm{ji}}\right]\left(\mathrm{e}_{\mathrm{i}} \otimes \mathrm{e}_{\mathrm{j}}\right) \\
& =\frac{1}{2}\left(\mathbf{S}+\mathbf{S}^{\mathbf{T}}\right)
\end{aligned}
$$

Então $(\underline{\mathbf{I}} \underline{\overline{\mathbf{I}}})$ determina a parte simétrica do tensor $\mathbf{S}$. 


\section{PRODUTOS TENSORIAIS ENVOLVENDO O TENSOR A}

Em materiais que possuem isotropia transversal, o tensor $\mathbf{A}$, em particular, é por definição formado pelo produto tensorial do versor a perpendicular ao plano local de isotropia transversal por ele mesmo [CURNIER, HE \& ZYSSET (1995)].

$$
\mathbf{A}=\mathbf{a} \otimes \mathbf{a}=\mathrm{a}_{\mathrm{i}} \mathrm{a}_{\mathrm{j}}\left(\mathrm{e}_{\mathrm{i}} \otimes \mathrm{e}_{\mathrm{j}}\right)=\mathbf{a} \mathbf{a}^{\mathbf{T}}
$$

Como genericamente pode-se escrever:

$\mathbf{A}=\mathrm{A}_{\mathrm{ij}}\left(\mathrm{e}_{\mathrm{i}} \otimes \mathrm{e}_{\mathrm{j}}\right)$

segue que $A_{i j}=a_{i} a_{j}$.

Com base nas definições (1) à (4) decorrem os tensores de quarta ordem envolvidos na formulação do modelo proposto e que dependem do tensor $\mathbf{A}$ :

$\mathbf{A} \otimes \mathbf{A}=\mathrm{A}_{\mathrm{ij}} \mathrm{A}_{\mathrm{kl}}\left(\mathrm{e}_{\mathrm{i}} \otimes \mathrm{e}_{\mathrm{j}} \otimes \mathrm{e}_{\mathrm{k}} \otimes \mathrm{e}_{1}\right)$

$\mathbf{A} \otimes \mathbf{I}=\mathrm{A}_{\mathrm{ij}} \delta_{\mathrm{kl}}\left(\mathrm{e}_{\mathrm{i}} \otimes \mathrm{e}_{\mathrm{j}} \otimes \mathrm{e}_{\mathrm{k}} \otimes \mathrm{e}_{1}\right)$

$\mathbf{I} \otimes \mathbf{A}=\delta_{\mathrm{ij}} \mathrm{A}_{\mathrm{kl}}\left(\mathrm{e}_{\mathrm{i}} \otimes \mathrm{e}_{\mathrm{j}} \otimes \mathrm{e}_{\mathrm{k}} \otimes \mathrm{e}_{1}\right)$

$\mathbf{A} \otimes \underline{\mathbf{I}}=\mathrm{A}_{\mathrm{ik}} \delta_{\mathrm{jl}}\left(\mathrm{e}_{\mathrm{i}} \otimes \mathrm{e}_{\mathrm{j}} \otimes \mathrm{e}_{\mathrm{k}} \otimes \mathrm{e}_{1}\right)$

$\mathbf{A} \otimes \mathbf{I}=A_{i l} \delta_{j k}\left(e_{i} \otimes e_{j} \otimes e_{k} \otimes e_{1}\right)$

$\underline{\mathbf{I}} \otimes \mathbf{A}=\delta_{\mathrm{ik}} \mathrm{A}_{\mathrm{jl}}\left(\mathrm{e}_{\mathrm{i}} \otimes \mathrm{e}_{\mathrm{j}} \otimes \mathrm{e}_{\mathrm{k}} \otimes \mathrm{e}_{1}\right)$

$\mathbf{I} \otimes \mathbf{A}=\delta_{i 1} A_{j k}\left(e_{i} \otimes e_{j} \otimes e_{k} \otimes e_{1}\right)$

$\mathbf{A} \underline{\overline{\mathbf{I}}}=\frac{1}{2}\left(\mathrm{~A}_{\mathrm{ik}} \delta_{\mathrm{jl}}+\delta_{\mathrm{il}} \mathrm{A}_{\mathrm{jk}}\right)\left(\mathrm{e}_{\mathrm{i}} \otimes \mathrm{e}_{\mathrm{j}} \otimes \mathrm{e}_{\mathrm{k}} \otimes \mathrm{e}_{1}\right)$

$\underline{\mathbf{I}} \underline{\bar{A}}=\frac{1}{2}\left(\delta_{\mathrm{ik}} \mathrm{A}_{\mathrm{jl}}+\mathrm{A}_{\mathrm{il}} \delta_{\mathrm{jk}}\right)\left(\mathrm{e}_{\mathrm{i}} \otimes \mathrm{e}_{\mathrm{j}} \otimes \mathrm{e}_{\mathrm{k}} \otimes \mathrm{e}_{1}\right)$

As propriedades dos tensores relacionados acima são agora apresentadas considerando-se $\mathbf{S}$ como um tensor de segunda ordem. 
$(\mathbf{A} \otimes \mathbf{A}) \mathbf{S}=\mathrm{A}_{\mathrm{ij}} \mathrm{A}_{\mathrm{kl}} \mathrm{S}_{\mathrm{mn}}\left(\mathrm{e}_{\mathrm{i}} \otimes \mathrm{e}_{\mathrm{j}} \otimes \mathrm{e}_{\mathrm{k}} \otimes \mathrm{e}_{1}\right)\left(\mathrm{e}_{\mathrm{m}} \otimes \mathrm{e}_{\mathrm{n}}\right)$

$$
\begin{aligned}
& =\mathrm{A}_{\mathrm{ij}} \mathrm{A}_{\mathrm{kl}} \mathrm{S}_{\mathrm{mn}} \delta_{\mathrm{km}} \delta_{\ln }\left(\mathrm{e}_{\mathrm{i}} \otimes \mathrm{e}_{\mathrm{j}}\right) \\
& =\mathrm{A}_{\mathrm{ij}} \mathrm{A}_{\mathrm{kl}} \mathrm{S}_{\mathrm{kl}}\left(\mathrm{e}_{\mathrm{i}} \otimes \mathrm{e}_{\mathrm{j}}\right) \\
& =(\mathbf{S} \cdot \mathbf{A}) \mathbf{A}
\end{aligned}
$$

$(\mathbf{A} \otimes \mathbf{I}) \mathbf{S}=\mathrm{A}_{\mathrm{ij}} \delta_{\mathrm{kl}} \mathrm{S}_{\mathrm{mn}}\left(\mathrm{e}_{\mathrm{i}} \otimes \mathrm{e}_{\mathrm{j}} \otimes \mathrm{e}_{\mathrm{k}} \otimes \mathrm{e}_{1}\right)\left(\mathrm{e}_{\mathrm{m}} \otimes \mathrm{e}_{\mathrm{n}}\right)$

$$
\begin{aligned}
& =\mathrm{A}_{\mathrm{ij}} \delta_{\mathrm{kl}} \mathrm{S}_{\mathrm{mn}} \delta_{\mathrm{km}} \delta_{1 \mathrm{ln}}\left(\mathrm{e}_{\mathrm{i}} \otimes \mathrm{e}_{\mathrm{j}}\right) \\
& =\mathrm{A}_{\mathrm{ij}} \delta_{\mathrm{kl}} \mathrm{S}_{\mathrm{kl}}\left(\mathrm{e}_{\mathrm{i}} \otimes \mathrm{e}_{\mathrm{j}}\right) \\
& =\mathrm{A}_{\mathrm{ij}} \mathrm{S}_{11}\left(\mathrm{e}_{\mathrm{i}} \otimes \mathrm{e}_{\mathrm{j}}\right) \\
& =(\operatorname{tr} \mathbf{S}) \mathbf{A} \\
& =(\mathbf{S} \cdot \mathbf{I}) \mathbf{A}
\end{aligned}
$$

$$
\begin{aligned}
(\mathbf{I} \otimes \mathbf{A}) \mathbf{S} & =\delta_{\mathrm{ij}} \mathrm{A}_{\mathrm{kl}} \mathrm{S}_{\mathrm{mn}}\left(\mathrm{e}_{\mathrm{i}} \otimes \mathrm{e}_{\mathrm{j}} \otimes \mathrm{e}_{\mathrm{k}} \otimes \mathrm{e}_{1}\right)\left(\mathrm{e}_{\mathrm{m}} \otimes \mathrm{e}_{\mathrm{n}}\right) \\
& =\delta_{\mathrm{ij}} \mathrm{A}_{\mathrm{kl}} \mathrm{S}_{\mathrm{mn}} \delta_{\mathrm{km}} \delta_{\ln }\left(\mathrm{e}_{\mathrm{i}} \otimes \mathrm{e}_{\mathrm{j}}\right) \\
& =\delta_{\mathrm{ij}} \mathrm{A}_{\mathrm{kl}} \mathrm{S}_{\mathrm{kl}}\left(\mathrm{e}_{\mathrm{i}} \otimes \mathrm{e}_{\mathrm{j}}\right) \\
& =(\mathbf{S} \cdot \mathbf{A}) \mathbf{I}
\end{aligned}
$$

$$
\begin{aligned}
& (\mathbf{A} \otimes \mathbf{I}) \mathbf{S}=\mathrm{A}_{\mathrm{ik}} \delta_{\mathrm{jl}} \mathrm{S}_{\mathrm{mn}}\left(\mathrm{e}_{\mathrm{i}} \otimes \mathrm{e}_{\mathrm{j}} \otimes \mathrm{e}_{\mathrm{k}} \otimes \mathrm{e}_{1}\right)\left(\mathrm{e}_{\mathrm{m}} \otimes \mathrm{e}_{\mathrm{n}}\right) \\
& =\mathrm{A}_{\mathrm{ik}} \delta_{\mathrm{jl}} \mathrm{S}_{\mathrm{mn}} \delta_{\mathrm{km}} \delta_{\ln }\left(\mathrm{e}_{\mathrm{i}} \otimes \mathrm{e}_{\mathrm{j}}\right) \\
& =\mathrm{A}_{\mathrm{ik}} \delta_{\mathrm{jl}} \mathrm{S}_{\mathrm{kl}}\left(\mathrm{e}_{\mathrm{i}} \otimes \mathrm{e}_{\mathrm{j}}\right) \\
& =\mathrm{A}_{\mathrm{ik}} \mathrm{S}_{\mathrm{kj}}\left(\mathrm{e}_{\mathrm{i}} \otimes \mathrm{e}_{\mathrm{j}}\right) \\
& =\mathbf{A S}
\end{aligned}
$$

$$
\begin{aligned}
(\mathbf{I} \otimes \mathbf{A}) \mathbf{S} & =\delta_{\mathrm{il}} \mathrm{A}_{\mathrm{jk}} \mathrm{S}_{\mathrm{mn}}\left(\mathrm{e}_{\mathrm{i}} \otimes \mathrm{e}_{\mathrm{j}} \otimes \mathrm{e}_{\mathrm{k}} \otimes \mathrm{e}_{1}\right)\left(\mathrm{e}_{\mathrm{m}} \otimes \mathrm{e}_{\mathrm{n}}\right) \\
& =\delta_{\mathrm{il}} \mathrm{A}_{\mathrm{jk}} \mathrm{S}_{\mathrm{mn}} \delta_{\mathrm{km}} \delta_{\ln }\left(\mathrm{e}_{\mathrm{i}} \otimes \mathrm{e}_{\mathrm{j}}\right) \\
& =\delta_{\mathrm{il}} \mathrm{A}_{\mathrm{jk}} \mathrm{S}_{\mathrm{kl}}\left(\mathrm{e}_{\mathrm{i}} \otimes \mathrm{e}_{\mathrm{j}}\right)
\end{aligned}
$$




$$
\begin{aligned}
& =\mathrm{A}_{\mathrm{jk}} \mathrm{S}_{\mathrm{ki}}\left(\mathrm{e}_{\mathrm{i}} \otimes \mathrm{e}_{\mathrm{j}}\right) \\
& =\mathbf{S}^{\mathbf{T}} \mathbf{A}^{\mathbf{T}}
\end{aligned}
$$

$$
\begin{aligned}
(\mathbf{A} \otimes \mathbf{I}) \mathbf{S} & =\frac{1}{2}\left(\mathrm{~A}_{\mathrm{ik}} \delta_{\mathrm{jl}}+\delta_{\mathrm{il}} \mathrm{A}_{\mathrm{jk}}\right) \mathrm{S}_{\mathrm{mn}}\left(\mathrm{e}_{\mathrm{i}} \otimes \mathrm{e}_{\mathrm{j}} \otimes \mathrm{e}_{\mathrm{k}} \otimes \mathrm{e}_{1}\right)\left(\mathrm{e}_{\mathrm{m}} \otimes \mathrm{e}_{\mathrm{n}}\right) \\
& =\frac{1}{2}\left(\mathrm{~A}_{\mathrm{ik}} \delta_{\mathrm{jl}}+\delta_{\mathrm{il}} \mathrm{A}_{\mathrm{jk}}\right) \mathrm{S}_{\mathrm{mn}} \delta_{\mathrm{km}} \delta_{\ln }\left(\mathrm{e}_{\mathrm{i}} \otimes \mathrm{e}_{\mathrm{j}}\right) \\
& =\frac{1}{2}\left(\mathrm{~A}_{\mathrm{ik}} \delta_{\mathrm{jl}} \mathrm{S}_{\mathrm{mn}} \delta_{\mathrm{km}} \delta_{\mathrm{ln}}+\delta_{\mathrm{il}} \mathrm{A}_{\mathrm{jk}} \mathrm{S}_{\mathrm{mn}} \delta_{\mathrm{km}} \delta_{\mathrm{ln}}\right)\left(\mathrm{e}_{\mathrm{i}} \otimes \mathrm{e}_{\mathrm{j}}\right) \\
& =\frac{1}{2}\left(\mathrm{~A}_{\mathrm{ik}} \delta_{\mathrm{jl}} \mathrm{S}_{\mathrm{kl}}+\delta_{\mathrm{il}} \mathrm{A}_{\mathrm{jk}} \mathrm{S}_{\mathrm{kl}}\right)\left(\mathrm{e}_{\mathrm{i}} \otimes \mathrm{e}_{\mathrm{j}}\right) \\
& =\frac{1}{2}\left(\mathrm{~A}_{\mathrm{ik}} \mathrm{S}_{\mathrm{kj}}+\mathrm{A}_{\mathrm{jk}} \mathrm{S}_{\mathrm{ki}}\right)\left(\mathrm{e}_{\mathrm{i}} \otimes \mathrm{e}_{\mathrm{j}}\right) \\
& =\frac{1}{2}[(\mathbf{A} \otimes \mathbf{I})+(\mathbf{I} \otimes \mathbf{A})] \mathrm{s} \\
& =\frac{1}{2}\left(\mathbf{A S}+\mathbf{S}^{\mathbf{T}} \mathbf{A}^{\mathbf{T}}\right)
\end{aligned}
$$

$$
\begin{aligned}
(\underline{\mathbf{I} \otimes \mathbf{A}}) \mathbf{S} & =\delta_{\mathrm{ik}} \mathrm{A}_{\mathrm{jl}} \mathrm{S}_{\mathrm{mn}}\left(\mathrm{e}_{\mathrm{i}} \otimes \mathrm{e}_{\mathrm{j}} \otimes \mathrm{e}_{\mathrm{k}} \otimes \mathrm{e}_{1}\right)\left(\mathrm{e}_{\mathrm{m}} \otimes \mathrm{e}_{\mathrm{n}}\right) \\
& =\delta_{\mathrm{ik}} \mathrm{A}_{\mathrm{jl}} \mathrm{S}_{\mathrm{mn}} \delta_{\mathrm{km}} \delta_{\ln }\left(\mathrm{e}_{\mathrm{i}} \otimes \mathrm{e}_{\mathrm{j}}\right) \\
& =\delta_{\mathrm{ik}} \mathrm{A}_{\mathrm{jl}} \mathrm{S}_{\mathrm{kl}}\left(\mathrm{e}_{\mathrm{i}} \otimes \mathrm{e}_{\mathrm{j}}\right) \\
& =\mathrm{A}_{\mathrm{jl}} \mathrm{S}_{\mathrm{il}}\left(\mathrm{e}_{\mathrm{i}} \otimes \mathrm{e}_{\mathrm{j}}\right) \\
& =\mathbf{S A}^{\mathbf{T}}
\end{aligned}
$$

$$
\begin{aligned}
(\mathbf{A} \otimes \mathbf{I}) \mathbf{S} & =A_{i l} \delta_{j k} S_{m n}\left(e_{i} \otimes e_{j} \otimes e_{k} \otimes e_{1}\right)\left(e_{m} \otimes e_{n}\right) \\
& =A_{i l} \delta_{j k} S_{m n} \delta_{k m} \delta_{l n}\left(e_{i} \otimes e_{j}\right) \\
& =A_{i l} \delta_{j k} S_{k l}\left(e_{i} \otimes e_{j}\right) \\
& =A_{i l} S_{j l}\left(e_{i} \otimes e_{j}\right) \\
& =\mathbf{A S} \mathbf{S}^{\mathbf{T}}
\end{aligned}
$$




$$
\begin{aligned}
(\underline{\mathbf{I} \otimes} \mathbf{A}) \mathbf{S} & =\frac{1}{2}\left(\delta_{\mathrm{ik}} \mathrm{A}_{\mathrm{jl}}+\mathrm{A}_{\mathrm{il}} \delta_{\mathrm{jk}}\right) \mathrm{S}_{\mathrm{mn}}\left(\mathrm{e}_{\mathrm{i}} \otimes \mathrm{e}_{\mathrm{j}} \otimes \mathrm{e}_{\mathrm{k}} \otimes \mathrm{e}_{1}\right)\left(\mathrm{e}_{\mathrm{m}} \otimes \mathrm{e}_{\mathrm{n}}\right) \\
& =\frac{1}{2}\left(\delta_{\mathrm{ik}} \mathrm{A}_{\mathrm{jl}}+\mathrm{A}_{\mathrm{il}} \delta_{\mathrm{jk}}\right) \mathrm{S}_{\mathrm{mn}} \delta_{\mathrm{km}} \delta_{\ln }\left(\mathrm{e}_{\mathrm{i}} \otimes \mathrm{e}_{\mathrm{j}}\right) \\
& =\frac{1}{2}\left(\delta_{\mathrm{ik}} \mathrm{A}_{\mathrm{jl}} \mathrm{S}_{\mathrm{mn}} \delta_{\mathrm{km}} \delta_{\mathrm{ln}}+\mathrm{A}_{\mathrm{il}} \delta_{\mathrm{jk}} \mathrm{S}_{\mathrm{mn}} \delta_{\mathrm{km}} \delta_{\mathrm{ln}}\right)\left(\mathrm{e}_{\mathrm{i}} \otimes \mathrm{e}_{\mathrm{j}}\right) \\
& =\frac{1}{2}\left(\delta_{\mathrm{ik}} \mathrm{A}_{\mathrm{jl}} \mathrm{S}_{\mathrm{kl}}+\mathrm{A}_{\mathrm{il}} \delta_{\mathrm{jk}} \mathrm{S}_{\mathrm{kl}}\right)\left(\mathrm{e}_{\mathrm{i}} \otimes \mathrm{e}_{\mathrm{j}}\right) \\
& =\frac{1}{2}\left(\mathrm{~A}_{\mathrm{jl}} \mathrm{S}_{\mathrm{il}}+\mathrm{A}_{\mathrm{il}} \mathrm{S}_{\mathrm{jl}}\right)\left(\mathrm{e}_{\mathrm{i}} \otimes \mathrm{e}_{\mathrm{j}}\right) \\
& =\frac{1}{2}[(\mathbf{I} \otimes \mathbf{A})+(\mathbf{A} \otimes \mathbf{I})] \\
& =\frac{1}{2}\left(\mathbf{S} \mathbf{A}^{\mathbf{T}}+\mathbf{A} \mathbf{S}^{\mathbf{T}}\right)
\end{aligned}
$$

Das propriedades dos tensores vistas até agora, pode-se, então, resumir as operações de aplicação de produtos tensoriais formadas por tensores quaisquer de segunda ordem descritas em CURNIER, HE \& ZYSSET (1995) e DEL PIERO (1979), como:

$[R \otimes \mathbf{T}] \mathbf{S}=(\mathbf{S . T}) \mathbf{R}$

$[\mathbf{R} \otimes \mathbf{T}] \mathbf{S}=\mathbf{R S T}^{\mathbf{T}} \quad[\mathbf{R} \otimes \bar{\otimes} \mathbf{T}] \mathbf{S}=\mathbf{R S}^{\mathbf{T}} \mathbf{T}^{\mathbf{T}}$

$[\mathbf{R} \bar{\otimes} \mathbf{T}] \mathbf{S}=\left(\mathbf{R S T}^{\mathbf{T}}+\mathbf{T S}^{\mathbf{T}} \mathbf{R}^{\mathbf{T}}\right) / 2$

sendo $\mathbf{R} \bar{\otimes}^{-} \mathbf{T}=[\mathbf{R} \otimes \mathbf{T}+\mathbf{T} \otimes \mathbf{R}] / 2$

\section{FORMA MATRICIAL DOS TENSORES DE INTERESSE}

Pode-se observar no capítulo 3 deste trabalho, que os tensores constitutivos do modelo proposto são constituídos pelos tensores de quarta ordem descritos no 
item anterior. Justifica-se, portanto, a apresentação em forma matricial destes, para melhor interpretação do seu emprego quando da implementação do modelo proposto em código de cálculo.

A partir de sua representação puramente matricial, compreende-se que os produtos tensoriais de (1) à (4) e de (10) à (18) possuem a função de alocar as constantes elásticas do material em determinadas posições nos tensores constitutivos.

Sendo assim, considere-se o caso em que num sistema de coordenadas locais a direção $\mathrm{X}$ seja perpendicular ao plano local de isotropia transversal $\mathrm{YZ}$ como descrito na figura 1.

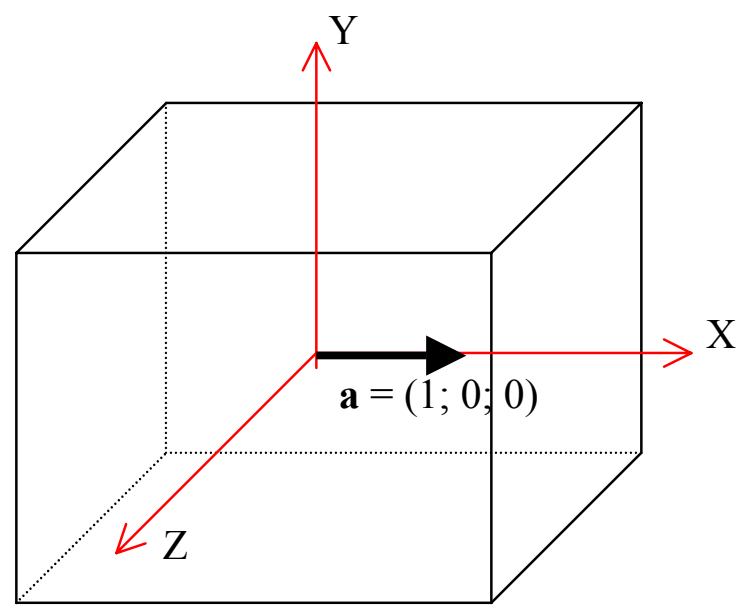

Figura 1 - Sistema local de referência do material.

$\mathrm{O}$ plano $\mathrm{YZ}$ coincide com o plano local de isotropia transversal.

Portanto, o tensor A resulta:

$\mathbf{A}=\mathbf{a a}^{\mathbf{T}}=\left\{\begin{array}{l}1 \\ 0 \\ 0\end{array}\right\}\left\{\begin{array}{lll}1 & 0 & 0\end{array}\right\}=\left[\begin{array}{lll}1 & 0 & 0 \\ 0 & 0 & 0 \\ 0 & 0 & 0\end{array}\right]=\left\{\begin{array}{l}1 \\ 0 \\ 0 \\ 0 \\ 0 \\ 0 \\ 0 \\ 0 \\ 0\end{array}\right\}$ 
Antes de apresentar a forma matricial dos tensores de quarta ordem, faz-se necessário mostrar a alocação dos componentes das matrizes representativas desses tensores. Logo, considerem-se dois tensores de segunda ordem genéricos $\mathbf{R}$ e $\mathbf{S}$ dados por:

$\mathbf{R}=\left[\begin{array}{lll}\mathrm{R}_{11} & \mathrm{R}_{12} & \mathrm{R}_{13} \\ \mathrm{R}_{21} & \mathrm{R}_{22} & \mathrm{R}_{23} \\ \mathrm{R}_{31} & \mathrm{R}_{32} & \mathrm{R}_{33}\end{array}\right]=\left\{\begin{array}{l}\mathrm{R}_{11} \\ \mathrm{R}_{22} \\ \mathrm{R}_{33} \\ \mathrm{R}_{12} \\ \mathrm{R}_{13} \\ \mathrm{R}_{21} \\ \mathrm{R}_{23} \\ \mathrm{R}_{31} \\ \mathrm{R}_{32}\end{array}\right\} \quad \mathbf{S}=\left[\begin{array}{lll}\mathrm{S}_{11} & \mathrm{~S}_{12} & \mathrm{~S}_{13} \\ \mathrm{~S}_{21} & \mathrm{~S}_{22} & \mathrm{~S}_{23} \\ \mathrm{~S}_{31} & \mathrm{~S}_{32} & \mathrm{~S}_{33}\end{array}\right]=\left\{\begin{array}{l}\mathrm{S}_{11} \\ \mathrm{~S}_{22} \\ \mathrm{~S}_{33} \\ \mathrm{~S}_{12} \\ \mathrm{~S}_{13} \\ \mathrm{~S}_{21} \\ \mathrm{~S}_{23} \\ \mathrm{~S}_{31} \\ \mathrm{~S}_{32}\end{array}\right\}$

O produto tensorial entre os tensores $\mathbf{R}$ e $\mathbf{S}$ gera um tensor de quarta de ordem dado, de uma forma geral, por:

$$
\left.\mathbf{R} \otimes \mathbf{S}=\mathbf{R S}^{\mathbf{T}}=\left\{\begin{array}{l}
\mathrm{R}_{11} \\
\mathrm{R}_{22} \\
\mathrm{R}_{33} \\
\mathrm{R}_{12} \\
\mathrm{R}_{13} \\
\mathrm{R}_{21} \\
\mathrm{R}_{23} \\
\mathrm{R}_{31} \\
\mathrm{R}_{32}
\end{array}\right\} \begin{array}{lllllllll}
\mathrm{S}_{11} & \mathrm{~S}_{22} & \mathrm{~S}_{33} & \mathrm{~S}_{12} & \mathrm{~S}_{13} & \mathrm{~S}_{21} & \mathrm{~S}_{23} & \mathrm{~S}_{31} & \mathrm{~S}_{32}
\end{array}\right\}
$$

Finalmente, a alocação dos componentes das matrizes representativas dos tensores de quarta ordem resulta da operação descrita acima gerando, portanto, tensores cuja forma matricial contem 9 linhas e 9 colunas somando 81 componentes.

Logo, observando o sistema de coordenadas descrito na figural, os tensores de quarta ordem envolvidos na formulação assumem as seguintes formas: 


$$
\mathbf{I} \otimes \mathbf{I}=\left[\begin{array}{ccccccccc}
1 & 1 & 1 & 0 & 0 & 0 & 0 & 0 & 0 \\
1 & 1 & 1 & 0 & 0 & 0 & 0 & 0 & 0 \\
1 & 1 & 1 & 0 & 0 & 0 & 0 & 0 & 0 \\
0 & 0 & 0 & 0 & 0 & 0 & 0 & 0 & 0 \\
0 & 0 & 0 & 0 & 0 & 0 & 0 & 0 & 0 \\
0 & 0 & 0 & 0 & 0 & 0 & 0 & 0 & 0 \\
0 & 0 & 0 & 0 & 0 & 0 & 0 & 0 & 0 \\
0 & 0 & 0 & 0 & 0 & 0 & 0 & 0 & 0 \\
0 & 0 & 0 & 0 & 0 & 0 & 0 & 0 & 0
\end{array}\right]
$$

$$
\overline{\mathbf{I}} \mathbf{I}=\left[\begin{array}{ccccccccc}
1 & 0 & 0 & 0 & 0 & 0 & 0 & 0 & 0 \\
0 & 1 & 0 & 0 & 0 & 0 & 0 & 0 & 0 \\
0 & 0 & 1 & 0 & 0 & 0 & 0 & 0 & 0 \\
0 & 0 & 0 & 0 & 0 & 1 & 0 & 0 & 0 \\
0 & 0 & 0 & 0 & 0 & 0 & 0 & 1 & 0 \\
0 & 0 & 0 & 1 & 0 & 0 & 0 & 0 & 0 \\
0 & 0 & 0 & 0 & 0 & 0 & 0 & 0 & 1 \\
0 & 0 & 0 & 0 & 1 & 0 & 0 & 0 & 0 \\
0 & 0 & 0 & 0 & 0 & 0 & 1 & 0 & 0
\end{array}\right]
$$




$$
\mathbf{A} \otimes \mathbf{A}=\left[\begin{array}{lllllllll}
1 & 0 & 0 & 0 & 0 & 0 & 0 & 0 & 0 \\
0 & 0 & 0 & 0 & 0 & 0 & 0 & 0 & 0 \\
0 & 0 & 0 & 0 & 0 & 0 & 0 & 0 & 0 \\
0 & 0 & 0 & 0 & 0 & 0 & 0 & 0 & 0 \\
0 & 0 & 0 & 0 & 0 & 0 & 0 & 0 & 0 \\
0 & 0 & 0 & 0 & 0 & 0 & 0 & 0 & 0 \\
0 & 0 & 0 & 0 & 0 & 0 & 0 & 0 & 0 \\
0 & 0 & 0 & 0 & 0 & 0 & 0 & 0 & 0 \\
0 & 0 & 0 & 0 & 0 & 0 & 0 & 0 & 0
\end{array}\right]
$$

$$
\mathbf{A} \otimes \mathbf{I}=\left[\begin{array}{lllllllll}
1 & 1 & 1 & 0 & 0 & 0 & 0 & 0 & 0 \\
0 & 0 & 0 & 0 & 0 & 0 & 0 & 0 & 0 \\
0 & 0 & 0 & 0 & 0 & 0 & 0 & 0 & 0 \\
0 & 0 & 0 & 0 & 0 & 0 & 0 & 0 & 0 \\
0 & 0 & 0 & 0 & 0 & 0 & 0 & 0 & 0 \\
0 & 0 & 0 & 0 & 0 & 0 & 0 & 0 & 0 \\
0 & 0 & 0 & 0 & 0 & 0 & 0 & 0 & 0 \\
0 & 0 & 0 & 0 & 0 & 0 & 0 & 0 & 0 \\
0 & 0 & 0 & 0 & 0 & 0 & 0 & 0 & 0
\end{array}\right]
$$

$$
\mathbf{I} \otimes \mathbf{A}=\left[\begin{array}{lllllllll}
1 & 0 & 0 & 0 & 0 & 0 & 0 & 0 & 0 \\
1 & 0 & 0 & 0 & 0 & 0 & 0 & 0 & 0 \\
1 & 0 & 0 & 0 & 0 & 0 & 0 & 0 & 0 \\
0 & 0 & 0 & 0 & 0 & 0 & 0 & 0 & 0 \\
0 & 0 & 0 & 0 & 0 & 0 & 0 & 0 & 0 \\
0 & 0 & 0 & 0 & 0 & 0 & 0 & 0 & 0 \\
0 & 0 & 0 & 0 & 0 & 0 & 0 & 0 & 0 \\
0 & 0 & 0 & 0 & 0 & 0 & 0 & 0 & 0 \\
0 & 0 & 0 & 0 & 0 & 0 & 0 & 0 & 0
\end{array}\right]
$$




$$
\mathbf{A} \underline{\bar{\otimes} \mathbf{I}}=\left[\begin{array}{ccccccccc}
1 & 0 & 0 & 0 & 0 & 0 & 0 & 0 & 0 \\
0 & 0 & 0 & 0 & 0 & 0 & 0 & 0 & 0 \\
0 & 0 & 0 & 0 & 0 & 0 & 0 & 0 & 0 \\
0 & 0 & 0 & \frac{1}{2} & 0 & 0 & 0 & 0 & 0 \\
0 & 0 & 0 & 0 & \frac{1}{2} & 0 & 0 & 0 & 0 \\
0 & 0 & 0 & \frac{1}{2} & 0 & 0 & 0 & 0 & 0 \\
0 & 0 & 0 & 0 & 0 & 0 & 0 & 0 & 0 \\
0 & 0 & 0 & 0 & \frac{1}{2} & 0 & 0 & 0 & 0 \\
0 & 0 & 0 & 0 & 0 & 0 & 0 & 0 & 0
\end{array}\right]
$$

$$
\underline{\mathbf{I} \otimes} \mathbf{A}=\left[\begin{array}{ccccccccc}
1 & 0 & 0 & 0 & 0 & 0 & 0 & 0 & 0 \\
0 & 0 & 0 & 0 & 0 & 0 & 0 & 0 & 0 \\
0 & 0 & 0 & 0 & 0 & 0 & 0 & 0 & 0 \\
0 & 0 & 0 & 0 & 0 & \frac{1}{2} & 0 & 0 & 0 \\
0 & 0 & 0 & 0 & 0 & 0 & 0 & \frac{1}{2} & 0 \\
0 & 0 & 0 & 0 & 0 & \frac{1}{2} & 0 & 0 & 0 \\
0 & 0 & 0 & 0 & 0 & 0 & 0 & 0 & 0 \\
0 & 0 & 0 & 0 & 0 & 0 & 0 & \frac{1}{2} & 0 \\
0 & 0 & 0 & 0 & 0 & 0 & 0 & 0 & 0
\end{array}\right]
$$

$$
(\mathbf{A} \otimes \mathbf{I})+(\mathbf{I} \otimes \mathbf{A})=\left[\begin{array}{ccccccccc}
2 & 1 & 1 & 0 & 0 & 0 & 0 & 0 & 0 \\
1 & 0 & 0 & 0 & 0 & 0 & 0 & 0 & 0 \\
1 & 0 & 0 & 0 & 0 & 0 & 0 & 0 & 0 \\
0 & 0 & 0 & 0 & 0 & 0 & 0 & 0 & 0 \\
0 & 0 & 0 & 0 & 0 & 0 & 0 & 0 & 0 \\
0 & 0 & 0 & 0 & 0 & 0 & 0 & 0 & 0 \\
0 & 0 & 0 & 0 & 0 & 0 & 0 & 0 & 0 \\
0 & 0 & 0 & 0 & 0 & 0 & 0 & 0 & 0 \\
0 & 0 & 0 & 0 & 0 & 0 & 0 & 0 & 0
\end{array}\right]
$$




$$
(\mathbf{A} \underline{\bar{\otimes} \mathbf{I}})+(\mathbf{I} \underline{\otimes} \mathbf{A})=\left[\begin{array}{ccccccccc}
2 & 0 & 0 & 0 & 0 & 0 & 0 & 0 & 0 \\
0 & 0 & 0 & 0 & 0 & 0 & 0 & 0 & 0 \\
0 & 0 & 0 & 0 & 0 & 0 & 0 & 0 & 0 \\
0 & 0 & 0 & \frac{1}{2} & 0 & \frac{1}{2} & 0 & 0 & 0 \\
0 & 0 & 0 & 0 & \frac{1}{2} & 0 & 0 & \frac{1}{2} & 0 \\
0 & 0 & 0 & \frac{1}{2} & 0 & \frac{1}{2} & 0 & 0 & 0 \\
0 & 0 & 0 & 0 & 0 & 0 & 0 & 0 & 0 \\
0 & 0 & 0 & 0 & \frac{1}{2} & 0 & 0 & \frac{1}{2} & 0 \\
0 & 0 & 0 & 0 & 0 & 0 & 0 & 0 & 0
\end{array}\right]
$$

Vale ressaltar que os tensores isótropos de quarta ordem (34), (35) e (36) não mudam sua forma seja qual for a localização do plano de isotropia transversal dentro do sistema de coordenadas locais adotado. Outra importante constatação é que os tensores constituintes [(34) à (37), (42) e (43)] do tensor constitutivo do modelo proposto são simétricos.

Considere-se agora o caso em que o versor a perpendicular ao plano local de isotropia transversal está contido no plano XY e possui uma inclinação $\alpha$ em relação ao eixo X segundo o sistema de coordenadas locais XYZ (Fig. 2).

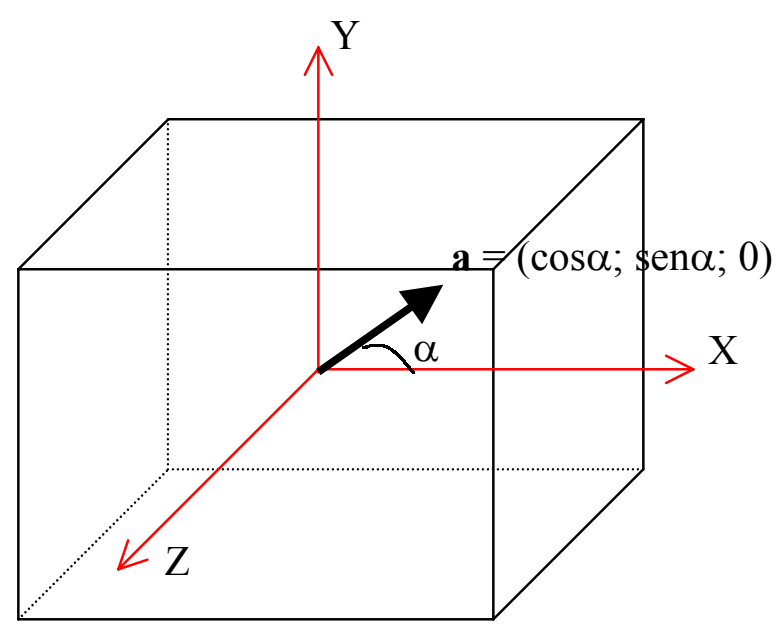

Figura 2 - Sistema local de referência do material. 
Portanto, o plano local de isotropia transversal rotaciona em trono do eixo $\mathrm{Z}$ de acordo com a variação do ângulo $\alpha$. Sendo assim, a forma matricial do tensor $\mathbf{A}$ é dada por:

$$
\begin{aligned}
& \mathbf{A}=\mathbf{a} \otimes \mathbf{a}=\mathbf{a a}^{\mathbf{T}}=\left\{\begin{array}{c}
\cos \alpha \\
\operatorname{sen} \alpha \\
0
\end{array}\right\}\left\{\begin{array}{lll}
\cos \alpha & \operatorname{sen} \alpha & 0
\end{array}\right\} \\
& \mathbf{A}=\left[\begin{array}{ccc}
\cos ^{2} \alpha & \cos \alpha \operatorname{sen} \alpha & 0 \\
\cos \alpha \operatorname{sen} \alpha & \operatorname{sen}^{2} \alpha & 0 \\
0 & 0 & 0
\end{array}\right]=\left\{\begin{array}{c}
\cos ^{2} \alpha \\
\operatorname{sen}^{2} \alpha \\
0 \\
\cos \alpha \operatorname{sen} \alpha \\
0 \\
\cos \alpha \operatorname{sen} \alpha \\
0 \\
0 \\
0
\end{array}\right\}
\end{aligned}
$$

Os tensores isótropos são, por definição, os mesmos dados por (34), (35) e (36). Já os tensores de quarta ordem que dependem do tensor A são expressos a seguir:

$\mathbf{A} \otimes \mathbf{A}=$

$\left[\begin{array}{ccccccccc}\cos ^{4} \alpha & \cos ^{2} \alpha \operatorname{sen}^{2} \alpha & 0 & \cos ^{3} \alpha \operatorname{sen} \alpha & 0 & \cos ^{3} \alpha \operatorname{sen} \alpha & 0 & 0 & 0 \\ \cos ^{2} \alpha \operatorname{sen}^{2} \alpha & \operatorname{sen}^{4} \alpha & 0 & \cos \alpha \operatorname{sen}^{3} \alpha & 0 & \cos \alpha \operatorname{sen}^{3} \alpha & 0 & 0 & 0 \\ 0 & 0 & 0 & 0 & 0 & 0 & 0 & 0 & 0 \\ \cos ^{3} \alpha \operatorname{sen} \alpha & \cos \alpha \operatorname{sen}^{3} \alpha & 0 & \cos ^{2} \alpha \operatorname{sen}^{2} \alpha & 0 & \cos ^{2} \alpha \operatorname{sen}^{2} \alpha & 0 & 0 & 0 \\ 0 & 0 & 0 & 0 & 0 & 0 & 0 & 0 & 0 \\ \cos ^{3} \alpha \operatorname{sen} \alpha & \cos \alpha \operatorname{sen}^{3} \alpha & 0 & \cos ^{2} \alpha \operatorname{sen}^{2} \alpha & 0 & \cos ^{2} \alpha \operatorname{sen}^{2} \alpha & 0 & 0 & 0 \\ 0 & 0 & 0 & 0 & 0 & 0 & 0 & 0 & 0 \\ 0 & 0 & 0 & 0 & 0 & 0 & 0 & 0 & 0 \\ 0 & 0 & 0 & 0 & 0 & 0 & 0 & 0 & 0\end{array}\right]$




$$
\mathbf{A} \otimes \mathbf{I}=\left[\begin{array}{ccccccccc}
\cos ^{2} \alpha & \cos ^{2} \alpha & \cos ^{2} \alpha & 0 & 0 & 0 & 0 & 0 & 0 \\
\operatorname{sen}^{2} \alpha & \operatorname{sen}^{2} \alpha & \operatorname{sen}^{2} \alpha & 0 & 0 & 0 & 0 & 0 & 0 \\
0 & 0 & 0 & 0 & 0 & 0 & 0 & 0 & 0 \\
\cos \alpha \operatorname{sen} \alpha & \cos \alpha \operatorname{sen} \alpha & \cos \alpha \operatorname{sen} \alpha & 0 & 0 & 0 & 0 & 0 & 0 \\
0 & 0 & 0 & 0 & 0 & 0 & 0 & 0 & 0 \\
\cos \alpha \operatorname{sen} \alpha & \cos \alpha \operatorname{sen} \alpha & \cos \alpha \operatorname{sen} \alpha & 0 & 0 & 0 & 0 & 0 & 0 \\
0 & 0 & 0 & 0 & 0 & 0 & 0 & 0 & 0 \\
0 & 0 & 0 & 0 & 0 & 0 & 0 & 0 & 0 \\
0 & 0 & 0 & 0 & 0 & 0 & 0 & 0 & 0
\end{array}\right]
$$

$\mathbf{I} \otimes \mathbf{A}=\left[\begin{array}{ccccccccc}\cos ^{2} \alpha & \operatorname{sen}^{2} \alpha & 0 & \cos \alpha \operatorname{sen} \alpha & 0 & \cos \alpha \operatorname{sen} \alpha & 0 & 0 & 0 \\ \cos ^{2} \alpha & \operatorname{sen}^{2} \alpha & 0 & \cos \alpha \operatorname{sen} \alpha & 0 & \cos \alpha \operatorname{sen} \alpha & 0 & 0 & 0 \\ \cos ^{2} \alpha & \operatorname{sen}^{2} \alpha & 0 & \cos \alpha \operatorname{sen} \alpha & 0 & \cos \alpha \operatorname{sen} \alpha & 0 & 0 & 0 \\ 0 & 0 & 0 & 0 & 0 & 0 & 0 & 0 & 0 \\ 0 & 0 & 0 & 0 & 0 & 0 & 0 & 0 & 0 \\ 0 & 0 & 0 & 0 & 0 & 0 & 0 & 0 & 0 \\ 0 & 0 & 0 & 0 & 0 & 0 & 0 & 0 & 0 \\ 0 & 0 & 0 & 0 & 0 & 0 & 0 & 0 & 0 \\ 0 & 0 & 0 & 0 & 0 & 0 & 0 & 0 & 0\end{array}\right]$

$\mathbf{A} \underline{\bar{\otimes} \mathbf{I}}=$

$\left[\begin{array}{ccccccccc}\cos ^{2} \alpha & 0 & 0 & 0 & 0 & \cos \alpha \operatorname{sen} \alpha & 0 & 0 & 0 \\ 0 & \operatorname{sen}^{2} \alpha & 0 & \cos \alpha \operatorname{sen} \alpha & 0 & 0 & 0 & 0 & 0 \\ 0 & 0 & 0 & 0 & 0 & 0 & 0 & 0 & 0 \\ \frac{\cos \alpha \operatorname{sen} \alpha}{2} & \frac{\cos \alpha \operatorname{sen} \alpha}{2} & 0 & \frac{\cos ^{2} \alpha}{2} & 0 & \frac{\operatorname{sen}^{2} \alpha}{2} & 0 & 0 & 0 \\ 0 & 0 & 0 & 0 & \frac{\cos ^{2} \alpha}{2} & 0 & \frac{\cos \alpha \operatorname{sen} \alpha}{2} & 0 & 0 \\ \frac{\cos \alpha \operatorname{sen} \alpha}{2} & \frac{\cos \alpha \operatorname{sen} \alpha}{2} & 0 & \frac{\cos ^{2} \alpha}{2} & 0 & \frac{\operatorname{sen}^{2} \alpha}{2} & 0 & 0 & 0 \\ 0 & 0 & 0 & 0 & \frac{\cos \alpha \operatorname{sen} \alpha}{2} & 0 & \frac{\operatorname{sen}^{2} \alpha}{2} & 0 & 0 \\ 0 & 0 & 0 & 0 & \frac{\cos 2}{2} & 0 & \frac{\cos \alpha \operatorname{sen} \alpha}{2} & 0 & 0 \\ 0 & 0 & 0 & 0 & \frac{\cos \alpha \operatorname{sen} \alpha}{2} & 0 & \frac{\operatorname{sen}^{2} \alpha}{2} & 0 & 0\end{array}\right]$


$\mathbf{I} \otimes \mathbf{A}=$

$\left[\begin{array}{ccccccccc}\cos ^{2} \alpha & 0 & 0 & \cos \alpha \mathbf{s e n} \alpha & 0 & 0 & 0 & 0 & 0 \\ 0 & \operatorname{sen}^{2} \alpha & 0 & 0 & 0 & \cos \alpha \operatorname{sen} \alpha & 0 & 0 & 0 \\ 0 & 0 & 0 & 0 & 0 & 0 & 0 & 0 & 0 \\ \frac{\cos \alpha \operatorname{sen} \alpha}{2} & \frac{\cos \alpha \operatorname{sen} \alpha}{2} & 0 & \frac{\operatorname{sen}^{2} \alpha}{2} & 0 & \frac{\cos ^{2} \alpha}{2} & 0 & 0 & 0 \\ 0 & 0 & 0 & 0 & 0 & 0 & 0 & \frac{\cos ^{2} \alpha}{2} & \frac{\cos \alpha \operatorname{sen} \alpha}{2} \\ \frac{\cos \alpha \operatorname{sen} \alpha}{2} & \frac{\cos \alpha \operatorname{sen} \alpha}{2} & 0 & \frac{\operatorname{sen}^{2} \alpha}{2} & 0 & \frac{\cos ^{2} \alpha}{2} & 0 & 0 & 0 \\ 0 & 0 & 0 & 0 & 0 & 0 & 0 & \frac{\cos \alpha \operatorname{sen} \alpha}{2} & \frac{\operatorname{sen}^{2} \alpha}{2} \\ 0 & 0 & 0 & 0 & 0 & 0 & 0 & \frac{\cos \alpha}{2} & \frac{\cos \alpha \operatorname{sen} \alpha}{2} \\ 0 & 0 & 0 & 0 & 0 & 0 & 0 & \frac{\cos \alpha \operatorname{sen} \alpha}{2} & \frac{\operatorname{sen}^{2} \alpha}{2}\end{array}\right]$

(49)

$(\mathbf{A} \otimes \mathbf{I})+(\mathbf{I} \otimes \mathbf{A})=$
$\left[\begin{array}{ccccccccc}2 \cos ^{2} \alpha & \cos ^{2} \alpha+\operatorname{sen}^{2} \alpha & \cos ^{2} \alpha & \cos \alpha \operatorname{sen} \alpha & 0 & \cos \alpha \operatorname{sen} \alpha & 0 & 0 & 0 \\ \cos ^{2} \alpha+\operatorname{sen}^{2} \alpha & 2 \operatorname{sen}^{2} \alpha & \operatorname{sen}^{2} \alpha & \cos \alpha \operatorname{sen} \alpha & 0 & \cos \alpha \operatorname{sen} \alpha & 0 & 0 & 0 \\ \cos ^{2} \alpha & \operatorname{sen}^{2} \alpha & 0 & \cos \alpha \operatorname{sen} \alpha & 0 & \cos \alpha \operatorname{sen} \alpha & 0 & 0 & 0 \\ \cos \alpha \operatorname{sen} \alpha & \cos \alpha \operatorname{sen} \alpha & \cos \alpha \operatorname{sen} \alpha & 0 & 0 & 0 & 0 & 0 & 0 \\ 0 & 0 & 0 & 0 & 0 & 0 & 0 & 0 & 0 \\ \cos \alpha \operatorname{sen} \alpha & \cos \alpha \operatorname{sen} \alpha & \cos \alpha \operatorname{sen} \alpha & 0 & 0 & 0 & 0 & 0 & 0 \\ 0 & 0 & 0 & 0 & 0 & 0 & 0 & 0 & 0 \\ 0 & 0 & 0 & 0 & 0 & 0 & 0 & 0 & 0 \\ 0 & 0 & 0 & 0 & 0 & 0 & 0 & 0 & 0\end{array}\right]$




$$
\begin{aligned}
& (\mathbf{A} \underline{\otimes} \mathbf{I})+(\mathbf{I} \bar{\otimes} \mathbf{A})=
\end{aligned}
$$

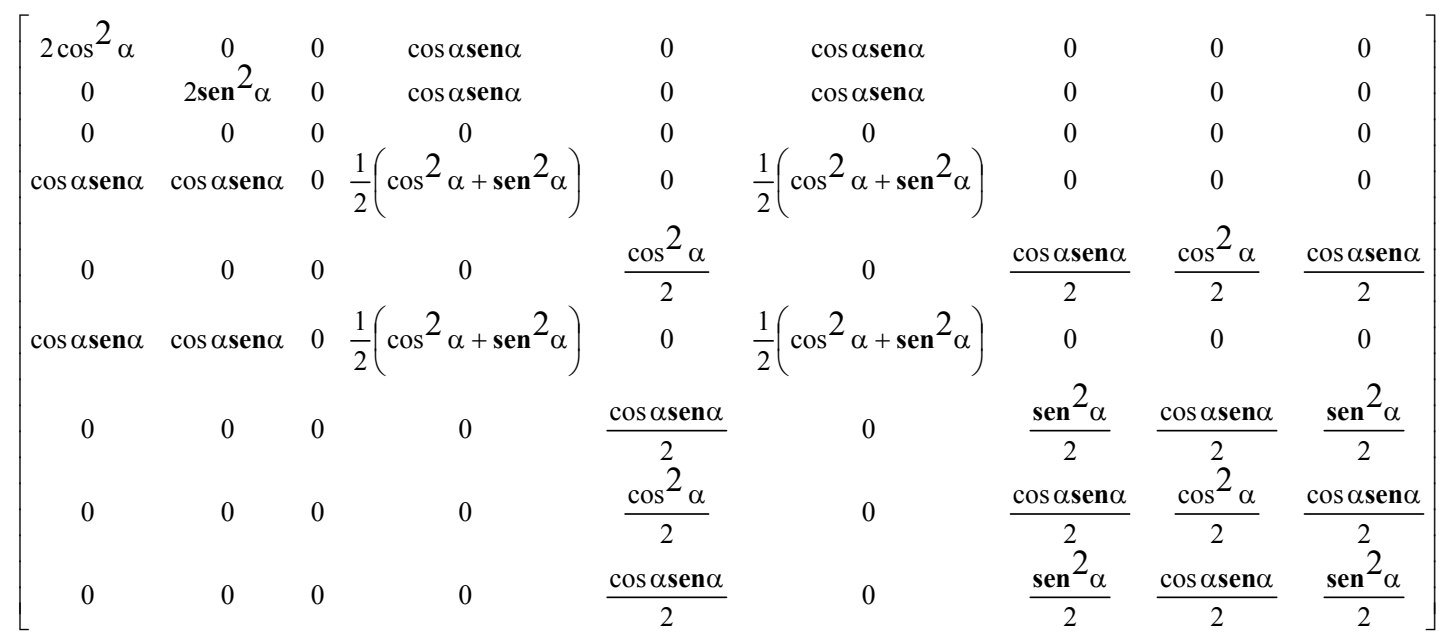

Mais uma vez observa-se que os tensores (45), (50) e (51), além dos tensores isótropos, são simétricos.

Cabe ressaltar que adotando-se $\alpha=0^{\circ}$ recuperam-se os tensores de quarta ordem dados por (37) à (43).

Finalmente, no caso mais geral, o versor a contém componentes segundo os eixos $\mathrm{X}, \mathrm{Y}$ e $\mathrm{Z}$ do sistema de coordenadas locais. Com isso, os tensores de quarta ordem contidos na formulação do modelo proposto possuem componentes dependentes dos cosenos e senos dos ângulos entre o versor a e os eixos X, Y e Z. 